\title{
Reducing nutrient pollution in water systems in China: challenges, trends and solutions
}

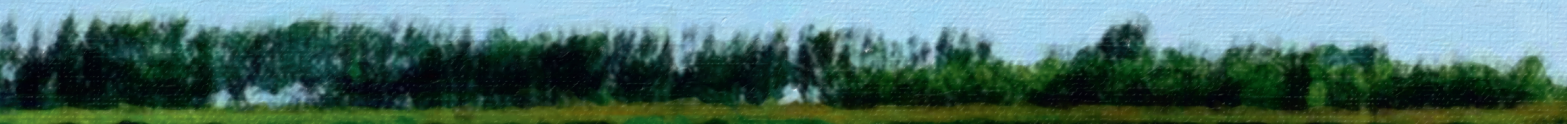
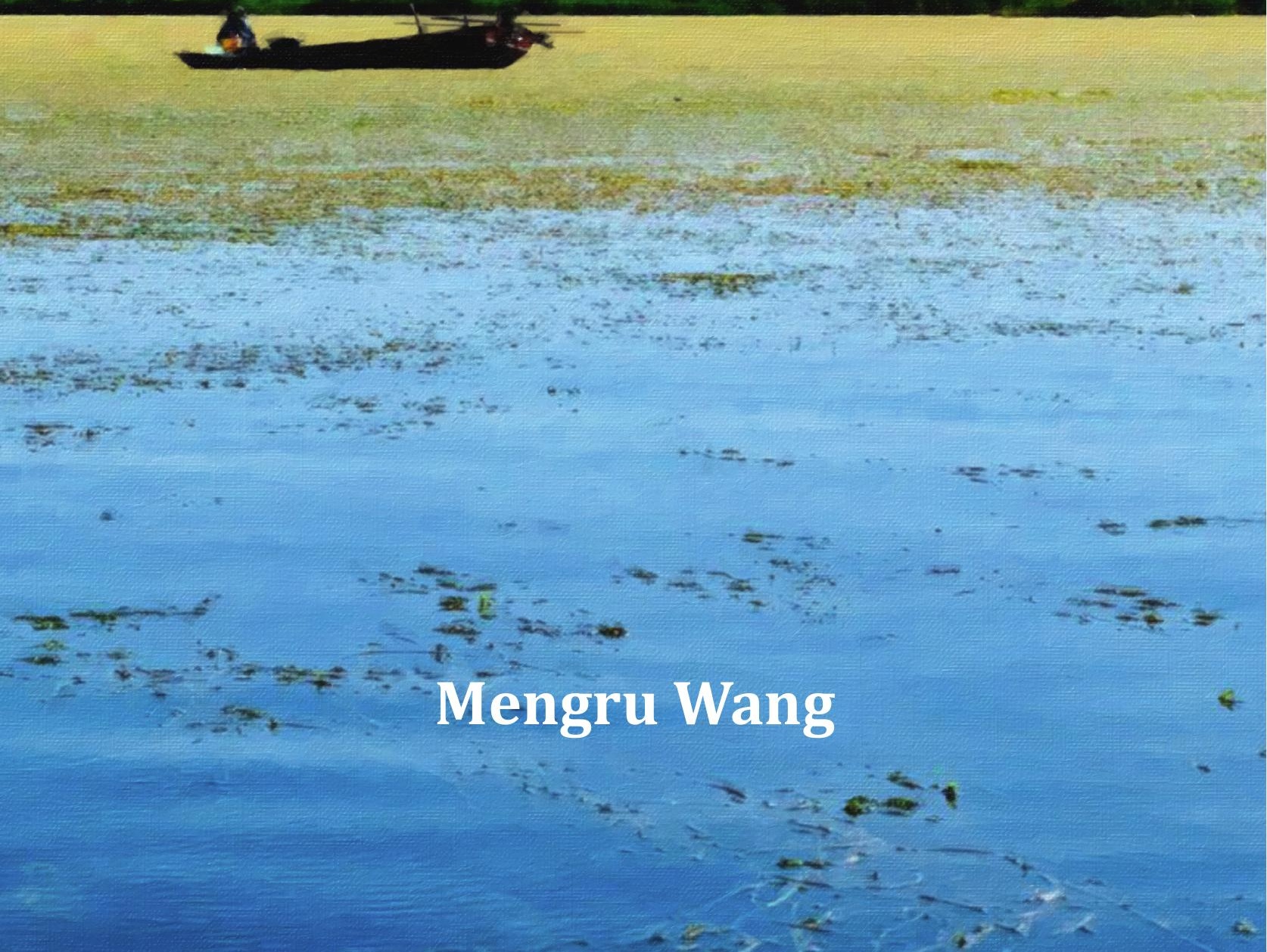



\section{Reducing nutrient pollution in water systems in China:}

challenges, trends and solutions

Mengru Wang 


\section{Thesis committee}

\section{Promotors}

Prof. Dr C. Kroeze

Professor of Water Systems and Global Change

Wageningen University \& Research

Prof. Dr L. Ma

Professor at the Center for Agricultural Resources Research, Institute of Genetic and Development Biology

Chinese Academy of Sciences, Hebei, China

\section{Other members}

Prof. Dr A.F. Bouwman, Utrecht University

Prof. Dr M. Flörke, Ruhr-University Bochum, Germany

Prof. Dr H.H.M. Rijnaarts, Wageningen University \& Research

Prof. Dr F. Zhang, China Agricultural University, Beijing, China

This research was conducted under the auspices of the Graduate School for SocioEconomic and Natural Sciences of the Environment (SENSE) 


\section{Reducing nutrient pollution in water systems in China:}

challenges, trends and solutions

\section{Mengru Wang}

\section{Thesis}

submitted in fulfilment of the requirements for the degree of doctor

at Wageningen University

by the authority of the Rector Magnificus,

Prof. Dr A.P.J. Mol,

in the presence of the

Thesis Committee appointed by the Academic Board

to be defended in public

on Wednesday 12 February 2020

at 4 p.m. in the Aula 


\section{Mengru Wang}

Reducing nutrient pollution in water systems in China: challenges, trends and solutions

255 pages

$\mathrm{PhD}$ thesis, Wageningen University, Wageningen, the Netherlands (2020)

With references, with summaries in English, Dutch and Chinese

ISBN 978-94-6395-197-5

DOI $10.18174 / 505611$ 
谨以此书献给我的父母

This book is dedicated to my parents 



\section{Table of Contents}

Chapter 1. General Introduction

Chapter 2. Hotspots for Nitrogen and Phosphorus Losses from Food Production in China: A County-Scale Analysis

Chapter 3. Excess Nutrient Loads to Lake Taihu: Opportunities for Nutrient Reduction

Chapter 4. Reactive Nitrogen Losses from China's Food System for the Shared Socio-economic Pathways (SSPs)

Chapter 5. Global Change Can Make Coastal Eutrophication Control in China More Difficult

Chapter 6. Exploring Nutrient Management Options to Increase Nitrogen and Phosphorus Use Efficiencies in Food Production of China

Chapter 7. Reducing Nutrient Pollution in Rivers and Coastal Waters in China: Implications for the Sustainable Development Goals

Chapter 8. Discussion and Conclusions

References

Appendices

Summary (English)

Samenvatting (Nederlands)

Summary (中文)

Acknowledgments

About the author

List of publications

SENSE Diploma

Chapters 2, 3, 4 and 6 have been published as peer-reviewed scientific articles. Chapter 5 has been resubmitted after revision based on positive reviews. Chapter 7 will be submitted for publication soon. The text, figures and tables of the published and submitted articles have been adjusted to the $\mathrm{PhD}$ thesis format. Editorial changes were made for reasons of uniformity of presentation. References should be made to the original articles. 



\section{Chapter 1. General Introduction}

\subsection{Background}

Earth's biogeochemical cycles of nitrogen (N) and phosphorus (P) have been strongly affected by anthropogenic activities in recent decades (Galloway et al., 2008; Lassaletta et al., 2016; Sutton et al., 2013; Sutton et al., 2011). At the global scale, the amount of $\mathrm{N}$ inputs to cropland increased fourfold between 1961 and 2009 (Lassaletta et al., 2016). The amount of P inputs to cropland increased threefold between 1950 and 2000 (Bouwman et al., 2013). Although $\mathrm{N}$ and $\mathrm{P}$ are essential for the growth of crops and animals (Galloway et al., 2017), only small fractions of the nutrient inputs to agriculture end up in the food products for consumption (Billen et al., 2013; Bouwman et al., 2013). A large part of the remainder is lost to the environment. This, together with increasing $\mathrm{N}$ and $\mathrm{P}$ discharge from urban waste (Morée et al., 2013; Van Drecht et al., 2009), has caused water pollution (leading to e.g., harmful algal blooms) in many regions of the world (Beusen et al., 2016; Fink et al., 2018; Seitzinger et al., 2010; UNEP, 2016b; Yasin et al., 2010). A better understanding of nutrient pollution in water systems as affected by human activities will help to identify solutions to water pollution.

\subsubsection{Water pollution by nutrients in China}

China has been developing fast in the last decades. These developments have affected $\mathrm{N}$ and $\mathrm{P}$ cycles in society and ecosystems. Excessive $\mathrm{N}$ and $\mathrm{P}$ are transported to rivers, lakes, and coastal waters, causing increasing nutrient pollution in these water systems.

Increasing nutrient pollution in Chinese rivers has been shown in many studies. Yu et al. (2019) found that critical surface-water quality standards have been exceeded in most provinces since the mid-1980s in China. In recent years, the situation has become worse (Bai et al., 2018a; Chen et al., 2019b; Strokal et al., 2016b; Tong et al., 2015; Xu et al., 2014). For example, Strokal et al. (2016b) showed that total dissolved N (TDN) and P (TDP) inputs to Chinese rivers increased 2- to 45 -fold between 1970 and 2000 as a result of intensive agricultural activities. Xu et al. (2014) found that $\mathrm{N}$ concentrations in many large rivers (e.g., the Huai, Hai, and Yellow rivers) exceeded the Chinese water quality standard for human consumption in the period of 2004-2009.

As a result of excessive nutrient loads to water systems, eutrophication in lakes has increased rapidly in China. Jin et al. (2005) indicated that the eutrophic area in the 34 
studied lakes increased from 5 to 55\% between 1978 to 1987. One example of these lakes is Lake Taihu, which is known to be the important water source for drinking, fisheries, irrigation, and other economic activities in its basin (Le et al., 2010). After the first report of algal blooms in 1987 in Lake Taihu, the situation of eutrophication has worsened (Chen et al., 2003; Duan et al., 2009; Janssen et al., 2017; Qin et al., 2010). Blooms of harmful algae are found every summer in the northern part of the lake (Qin et al., 2007b). This has largely decreased the clean and safe water availability for the local population (Guo, 2007). An example of this is Wuxi's city. This city uses water in Lake Taihu as its major drinking water source. The city had to shut down its drinking water station in 2007 because of the severe algal blooms in Lake Taihu (Qin et al., 2010).

Excessive nutrient inputs to surface waters also caused increasing river export of nutrients to coastal waters, resulting in coastal eutrophication. Strokal et al. (2016a) indicated that TDN and TDP export by six large Chinese rivers increased by a factor of 2 to 8 between 1970 and 2000. Studies on individual rivers such the Yangtze (Li et al., 2014a; Li et al., 2011a; Liu et al., 2018a; Tong et al., 2017; Yan et al., 2010), Yellow (Liu et al., 2012; Strokal et al., 2015; Tong et al., 2016) and Pearl (Hu and Li, 2009; Yin and Harrison, 2008) rivers also showed in general increasing river export of nutrients to sea in the last decades. As a result of increasing river export of nutrients to seas, more than 500 incidences of harmful algal blooms were reported between 2006 and 2012 in the coastal area of China (SOA, 2012). In 2012, the area affected by coastal eutrophication increased to $98,000 \mathrm{~km}^{2}$ (SOA, 2012).

The aforementioned studies illustrate the increasing nutrient pollution in water systems in China. This indicates a pressing need for China to explore solutions to this issue, for which a good understanding of the causes of nutrient pollution in water systems is required.

\subsubsection{Food production as an important cause}

There are several causes of nutrient pollution in water systems in China (Figure 1.1). Important causes are the rapid urbanization (e.g., sewage) and intensive food production (e.g., synthetic fertilizers, animal manure) (Gu et al., 2015; Ju et al., 2005; Ma et al., 2012b; Qu and Kroeze, 2012; Strokal et al., 2016a; Strokal et al., 2016b; Yu et al., 2019). Strokal et al. (2016a) indicated that, in 2000, more than two thirds of nutrient export by large Chinese rivers were from animal manure, synthetic fertilizers, and sewage. Other sources 
such as human waste in rural areas, as well as atmospheric $\mathrm{N}$ deposition on land and water bodies can also be important for local water systems (e.g., specific rivers or lakes) (Chadwick et al., 2015; Liu et al., 2011; Liu et al., 2013b; Xu et al., 2015b).

The construction of dams and reservoirs also affects nutrient transport from upstream to downstream of the rivers via nutrient retention (Liu et al., 2018a; Qu and Kroeze, 2010; Sun et al., 2013; Tong et al., 2016). For example, Liu et al. (2018a) revealed that the Three Gorges Reservoir in the Yangtze River has contributed between 5\% and 7\% of $\mathrm{N}$ and $\mathrm{P}$ retention in the whole basin. This has led to lower river export of $\mathrm{N}$ and $\mathrm{P}$ to the East China Sea, but higher risks for eutrophication in the reservoir, itself.

Among the abovementioned causes, food production is found to be one of the most important sources of water pollution in China. China has to feed one fifth of the world's population with less than $10 \%$ of the world's arable land (Larson, 2013). This, together with the rapid economic development, has intensified agricultural production in China. Grain production in China has increased fivefold in the last 60 years (Li et al., 2014b). Animal production began to transition to industrial sytems separate from crop production in the 1990s (Bai et al., 2014b; Chadwick et al., 2015). This stimulated intensive animal production in China. Livestock production in China has almost tripled from 142 to 441 million livestock units between 1980 and 2010 (Bai et al., 2018b). Around 40-80\% of pigs and poultry in 2010 were housed in industrial farms (MOA, 2011).

Crop and animal production in China is found to increase at a high environmental cost. One reason for this is the low nutrient use efficiency in crop production (Cui et al., 2010; Ma et al., 2010; Ma et al., 2012b; Zhang et al., 2012). In 2014, Chinese farmers were reported to apply, on average, $565 \mathrm{~kg} \mathrm{ha}^{-1}$ of synthetic fertilizers to their land (World Bank, 2016). This was much higher than in developed countries, such as the United States (138 $\mathrm{kg} \mathrm{ha}^{-1}$ ), in the same year (World Bank, 2016). This over-fertilization has led to a large amount of nutrients lost to water systems via leaching and runoff (Bai et al., 2016; Li et al., 2015a; Ma et al., 2012b; Zhang et al., 2013). Animal production has also become a large contributor to water pollution as a result of its industrialization. Industrial farms differ from traditional farms in manure management. In industrial farms, manure is often untreated and discharged to rivers as point sources, whereas manure in traditional farms is recycled on cropland as fertilizers (Chadwick et al., 2015). Due to such poor manure management in the industrial animal farms, direct discharge of animal manure has 
become a primary cause of water pollution in China in the 2000s (Ma et al., 2012b; Strokal et al., 2016b).

Considering these findings, it is important to understand how food production affects nutrient pollution in water systems in China. This understanding will contribute to exploring solutions to water pollution in China. 

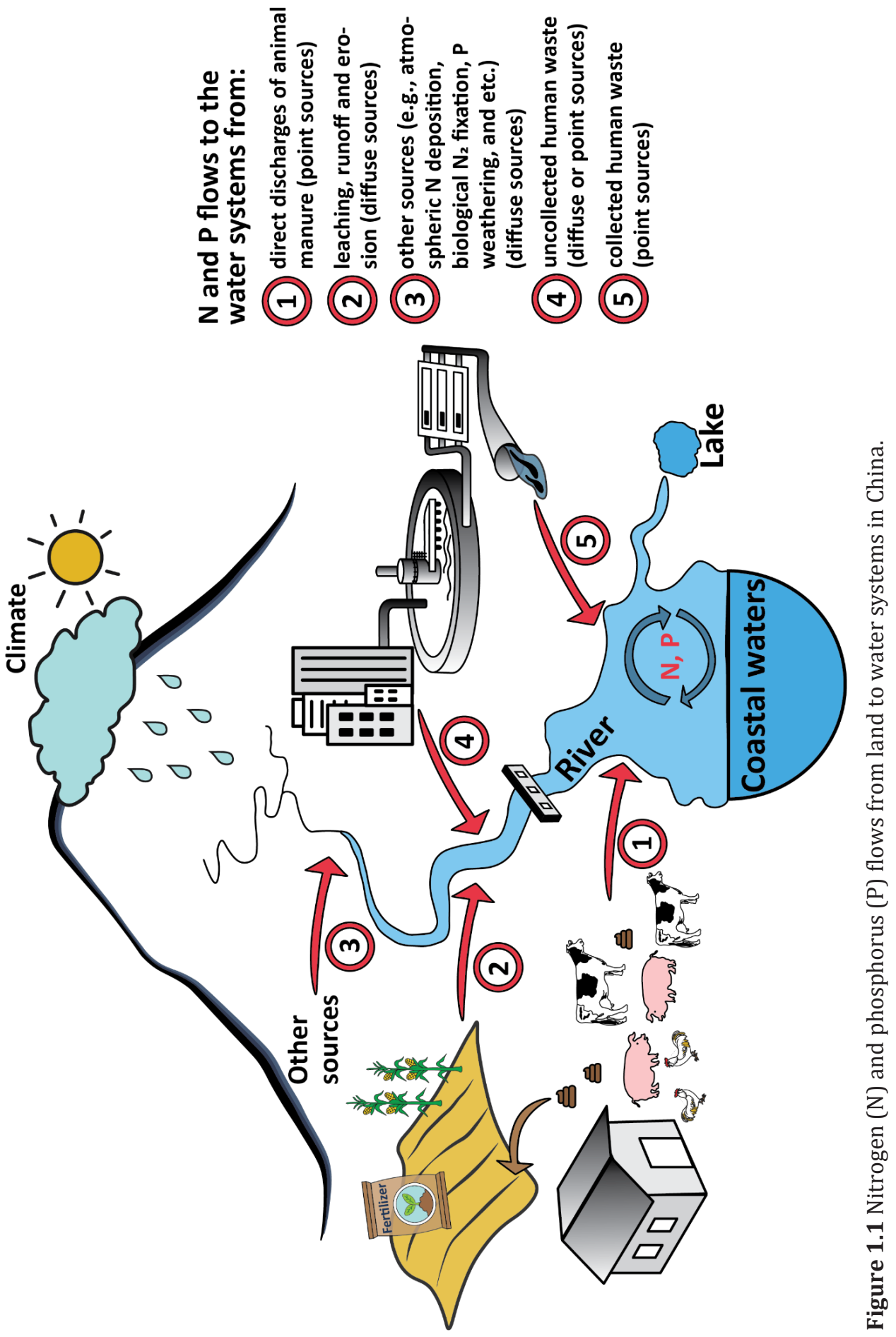


\subsubsection{Modeling the impact of food production on nutrient pollution in water systems}

Understanding both the state and the causes of water pollution is important for identifying effective solutions. Models can be used to improve our understanding of nutrient pollution in water systems as affected by human activities, and to explore solutions to this issue (Kroeze et al., 2012). As food production causes excessive nutrient loads to the environment (see Section 1.1.2), many models were developed to better understand nutrient flows in food production for China (Chen et al., 2008; Cui et al., 2013; Gu et al., 2015; Gu et al., 2012; Liu et al., 2004; Ma et al., 2012a; Ma et al., 2010; Ti et al., 2012) (see Table 1.1 for examples of these models). Most of these models focus on nutrient flows in the food production, itself, and thereby quantify nutrient losses to water systems at administrative scales (e.g., country, province). For example, Ma et al. (2012b) quantified the $\mathrm{N}$ and $\mathrm{P}$ budget of the crop and animal production system for Chinese provinces using NUFER (NUtrient flows in Food chains, Environment and Resources use). Based on the budget, $\mathrm{N}$ and $\mathrm{P}$ losses to the environment are estimated. This approach is transparent and provides information on the excessive nutrients in food production that can be lost to the water systems. However, limited insights are given on how nutrients in food production flow to waters (e.g., via leaching or runoff), their further transportation in water systems (e.g., transforming $\mathrm{N}$ and $\mathrm{P}$ from organic forms to inorganic forms and vice-versa), and the potential impacts on the water systems (e.g., eutrophication).

There are also models that quantify nutrient fluxes from terrestrial systems to water systems in China (Arnold et al., 2012; Beusen et al., 2016; Liu et al., 2019; Mayorga et al., 2010; Strokal et al., 2016a; UNEP, 2016b) (see Table 1.2 for examples of these models). These models quantify river export of nutrients taking into account human activities on land and nutrient retention in the water systems. Most of these models, however, lack spatially explicit information for human activities on land, particularly food production. For example, the MARINA 1.0 (Model to Assess River Inputs of Nutrients to seAs) model quantifies river export of nutrients to sea by sub-basins. It takes nutrient balance for agriculture based on national estimates (Bouwman et al., 2009; Bouwman et al., 2005; Mayorga et al., 2010). Nutrient balances at detailed administrative scales, such as Chinese counties, are relevant because the management of food production (e.g., use of synthetic fertilizers, recycling manure) is largely taking place at these scales. Analysis of nutrient flows in food production at the county scale can help to identify the hotspots for water 
pollution caused by human activities. Policies that target the hotspots at the administrative level (e.g., county) can be effective in reducing the pollution at the biogeophysical scale (e.g., river basins).

Based on the above, modeling studies that link nutrient flows in food production at administrative scales, and nutrient fluxes from land to water systems at bio-geophysical scales are still lacking for China. Such studies that link two types of models can simultaneously (1) provide quantitative information on nutrient pollution in water systems, and (2) improve the quantification of the spatial variabilities in the drivers and sources of nutrient pollution in water systems. Results of such studies will give an opportunity for exploring region-specific solutions to water pollution in China. 


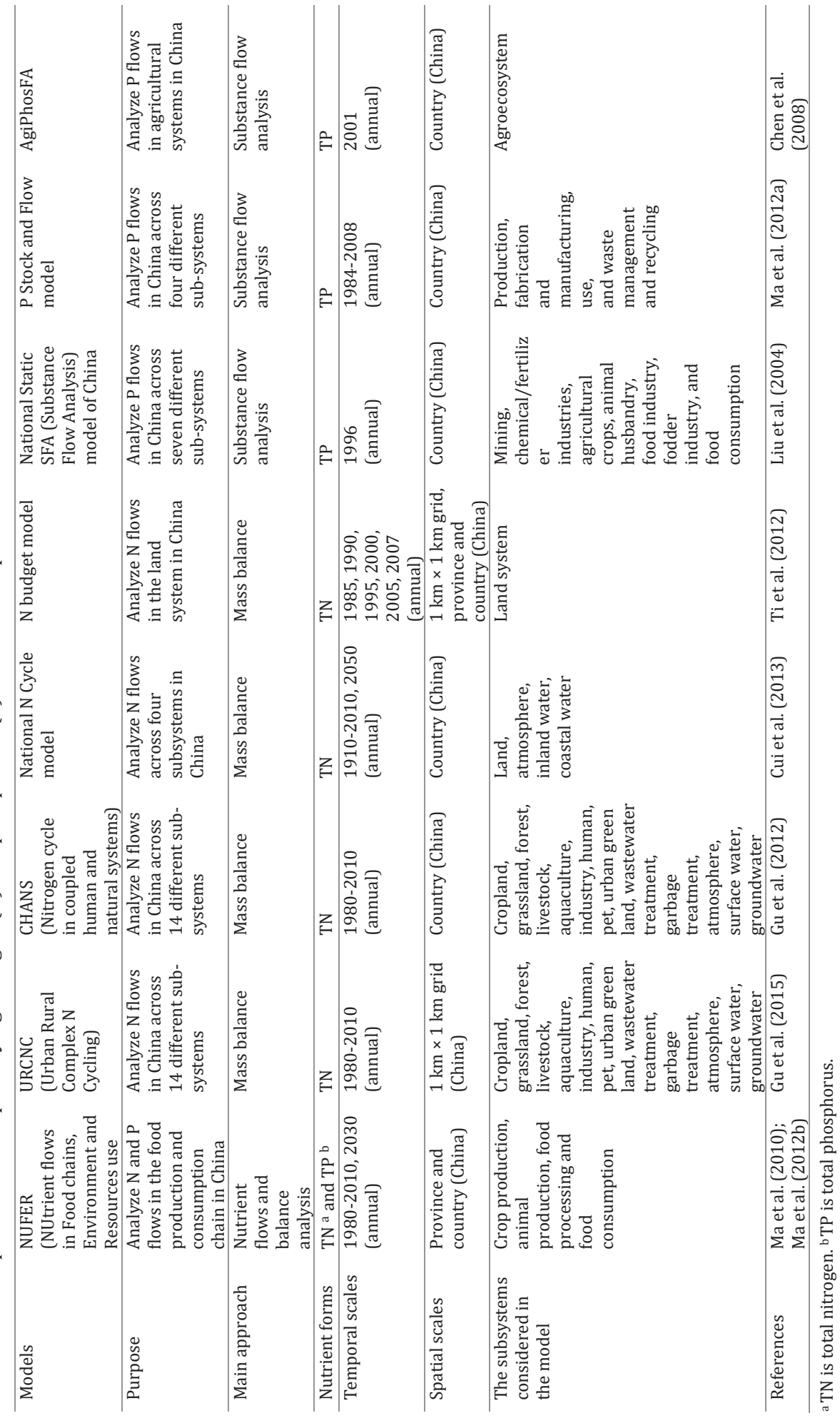




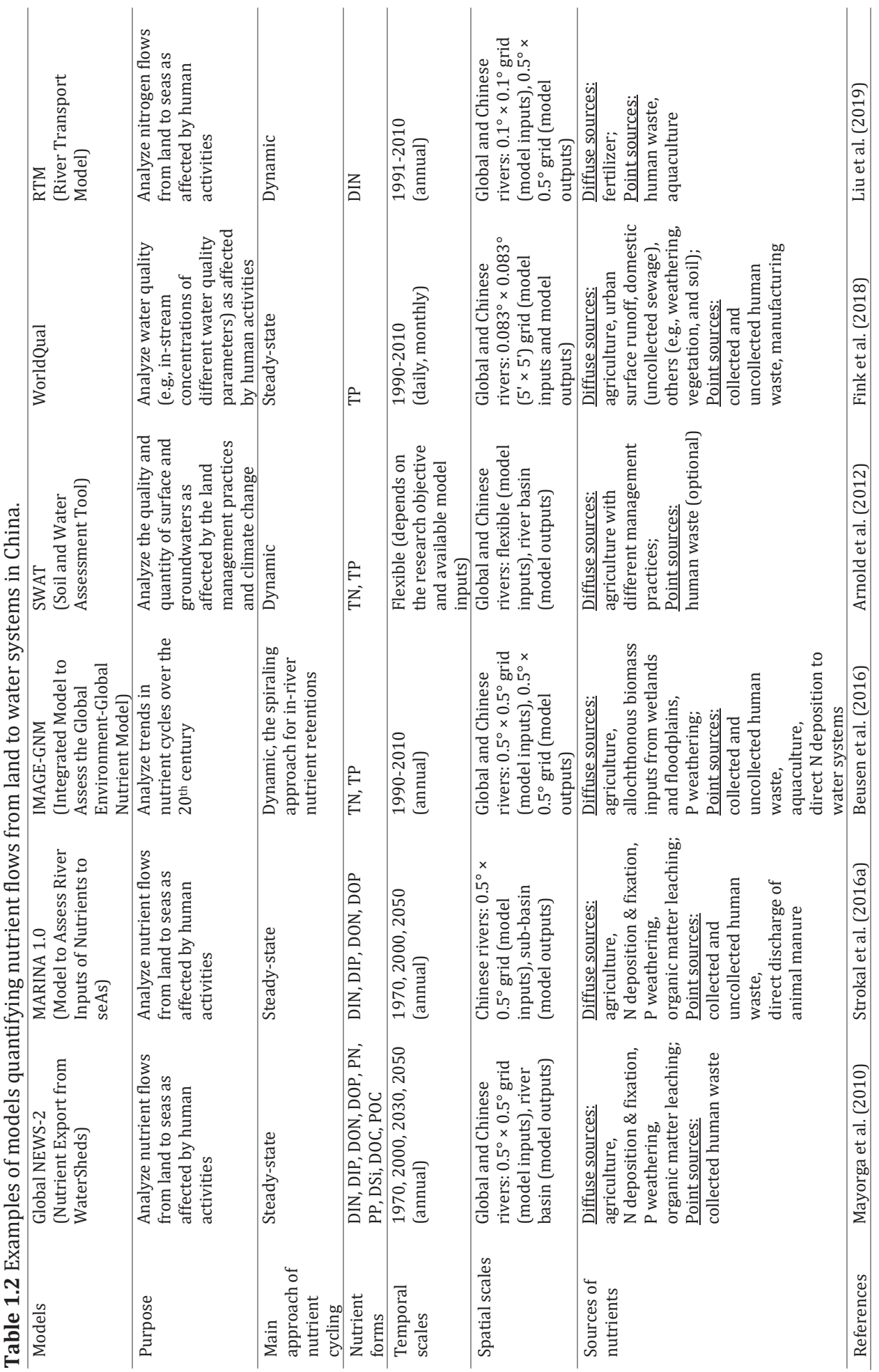




\subsubsection{Effects of global change on nutrient pollution in water systems}

Global change in the future may largely affect nutrient pollution in water systems. Socioeconomic development (e.g., changes in population, urbanization, or GDP) will lead to changes in food production and urbanization, and changes in the development of technologies for nutrient management (e.g., new technologies for manure and sewage treatment) (Jiang and O'Neill, 2017; O'Neill et al., 2017; Popp et al., 2017). All these changes will influence nutrient flows from human activities on land to water systems (Seitzinger et al., 2010; Van Puijenbroek et al., 2019; Van Puijenbroek et al., 2015).

Climate change will influence future hydrology (e.g., river discharge and runoff) (Lee et al., 2014; Sun et al., 2015a). Increases in runoff and river discharge may increase nutrient loads from land to rivers, and influence in-river nutrient retentions. This will in turn influence nutrient flows from land to seas (Howarth et al., 2006; Sinha et al., 2017). For example, Sinha et al. (2017) showed that climate change-induced changes in precipitation alone may increase river export of $\mathrm{N}$ to seas by rivers in the United States by $19 \pm 14 \%$ by the end of the century. To what extent climate change may affect nutrient pollution in water systems in China, however, is not well studied.

A few modeling studies exist to explore future trends in nutrient pollution in water systems for China via scenario analysis (Ma et al., 2013a; Qu and Kroeze, 2010; Strokal et al., 2017). These studies are based mainly on the storylines for future socio-economic development such as the Millennium Ecosystem Assessment (MEA) scenarios (Alcamo et al., 2005). Studies exploring how socio-economic and climatic changes together (global change) will affect water pollution by nutrients are lacking for China. For such analysis, the new global Shared Socio-economic Pathways (SSPs) (O'Neill et al., 2014) and Representative Concentration Pathways (RCPs) (Van Vuuren et al., 2011) can be used as the starting point. The SSPs are global storylines for future socio-economic development such as changes in gross domestic production, population, and urbanization ( $O^{\prime}$ Neill et al., 2014). The RCPs describe future climate change and cover a range of radiative forcing values of 2100 from 2.6 to $8.5 \mathrm{~W} \mathrm{~m}^{-2}$ (Van Vuuren et al., 2011). A better understanding of future trends as affected by SSPs and RCPs will provide a basis and help to identify potential solutions to water pollution in China under global change. 


\subsubsection{Reducing nutrient pollution to meet Sustainable Development Goals (SDGs)}

Water pollution has been gaining attention around the world. Many policies attempt to reduce water pollution to a safe level for society and ecosystems. An example is the introduction of the Sustainable Development Goals (SDGs) by the United Nations (UN) (United Nations, 2015). The SDGs include 17 goals divided into 169 targets for 2030 to achieve a better and sustainable future for the world. Among the 17 goals, two were specifically set for water quality: SDG 6 - "clean water and sanitation" and SDG 14 - "life below water". Some other SDGs are also linked to SDGs 6 and 14 for water quality through food production (SDG 2 - "zero hunger") and consumption (SDG 12 - "responsible consumption and production"), sewage management (SDG 11 - "sustainable cities and communities"), and climate change (SDG 13 - "climate action").

In order to meet the SDGs in China, the central government introduced "China's National Plan on Implementation of the 2030 Agenda for Sustainable Development" in 2016 (Li, 2016). This national plan sets detailed plans for reaching all SDGs including SDGs 6 and 14. The plans for SDGs 6 and 14 were developed based on existing Chinese environmental policies for water pollution such as the "Action Plan for Prevention and Control of Water Pollution" (SCPRC, 2015), and “Zero Growth in Synthetic Fertilizer Use from 2020" (MOA, 2015c). However, the assessment by Stiftung (2018) indicated that China is not on track towards reaching the SDGs in 2030 with current policies. This holds in particular for SDGs 6 and 14. Meanwhile, there is a lack of studies that assess the effectiveness of current policies on reducing water pollution in China. Such analysis can be useful for supporting future policies and meeting SDGs in China.

\subsection{Knowledge gaps and research objectives}

Based on the above, I identified the following knowledge gaps that prohibit China from addressing nutrient pollution in water systems. There is currently a limited understanding of:

1. how the spatial variability in food production at the county, provincial and national scales affects nutrient fluxes at bio-geophysical scales of water systems in China;

2. how global change affects future nutrient pollution in water systems in China; and

3. how effective current policies are in reducing water pollution in China in the future. 
Therefore, the objective of this $\mathrm{PhD}$ thesis is to improve our understanding of nutrient pollution in water systems in China, with a focus on the challenges, trends, and solutions. To this end, I formulated six sub-objectives for my research on nutrient pollution in water systems (sub-objectives 2, 4, and 6) from human activities on land (sub-objectives 1, 3, and 5; Figure 1.2).

Sub-objectives focusing on the challenges (Knowledge Gap 1):

1. To identify hotspots for nutrient losses from food production (Chapter 2)

2. To compare nutrient loads to Lake Taihu with the critical nutrient loads of the lake (Chapter 3, case study)

Sub-objectives focusing on the trends (Knowledge Gap 2):

3. To analyze how socio-economic development affects future nutrient losses from food production (Chapter 4)

4. To analyze how global change affects future river export of nutrients to seas (Chapter 5)

Sub-objectives focusing on the solutions (Knowledge Gap 3):

5. To explore how current and improved nutrient management affects future nutrient losses from food production (Chapter 6)

6. To explore future scenarios to reduce nutrient pollution in water systems and to meet the SDGs (Chapter 7) 


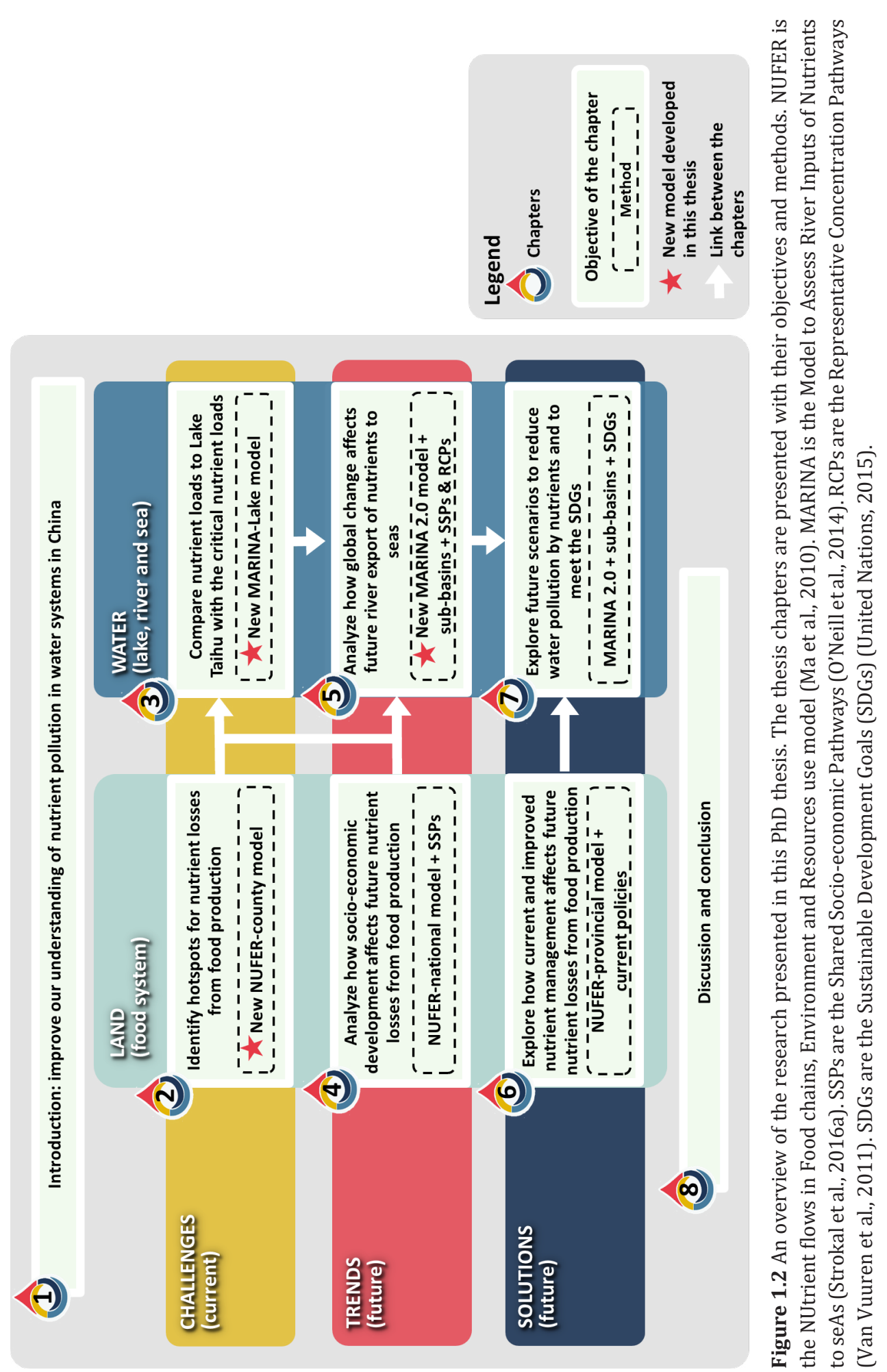




\subsection{Research approach}

To address the knowledge gaps identified in Section 1.2, I used the NUFER and MARINA models. I linked the NUFER and MARINA 1.0 models to better understand the impact of food production on nutrient pollution in water systems in China (Knowledge Gap 1) (Figure 1.3). NUFER was developed to analyze nutrient flows in food production at national and provincial scales (Ma et al., 2010; Ma et al., 2012b). Developing a new NUFER model for Chinese counties (NUFER-county) is needed, as governments' decisions on food production in China are often made for the counties. I used the results of NUFER-county as inputs to MARINA 1.0 to improve modeling of nutrient flows on land (a soft linking between NUFER and MARINA 1.0). This results in two new versions of MARINA: MARINA 2.0 and MARINA-Lake for China that combines the bio-geophysical (e.g., sub-basin) and administrative (e.g., county) scales for nutrient pollution in water systems (Figure 1.3).

I used the newly developed NUFER and MARINA models to explore future trends in water pollution by nutrients under global change (Knowledge Gap 2). I did this through scenario analysis based on the storylines of SSPs for socio-economic development, and of RCPs for climate change (Figure 1.3). The hydrology (e.g., water discharge, and runoff) in MARINA 1.0 is from WBM (Water Balance Model) (Fekete et al., 2010) and is not available for the RCPs. Therefore, I updated the hydrology in MARINA 2.0 and MARINA-Lake based on the VIC (Variable Infiltration Capacity) (Liang et al., 1994; Van Vliet et al., 2016a) and CWATM (Community WATer Model) (Burek et al., 2017) models (Figure 1.3).

I used the newly developed NUFER and MARINA models to analyze the effectiveness of current policies on reducing water pollution in China (Knowledge Gap 3). I did this through scenarios analysis. Scenarios incorporating current Chinese fertilizer policies and alternative nutrient management options were developed (Figure 1.3). Implementing these scenarios in the linked NUFER-MARINA model system provides quantitative information on the effectiveness of the policies and other potential options in reducing water pollution in China.

I used several indicators in this thesis to interpret the modeling results on nutrient pollution in water systems, and to assess SDGs 6 and 14 for water quality. The indicators are nutrient use efficiency in food production (Chapters 2, 4, 6), critical nutrient loads for lakes (Chapter 3), Chinese water quality standards (Chapter 5), and Indicator for Coastal Eutrophication Potential (ICEP; Chapters 5, 7). In Chapter 8, I discussed the strengths and 
weaknesses of using these indicators based on the Drivers, Pressure, State, Impact, and Response (DPSIR) framework (Kristensen, 2004).
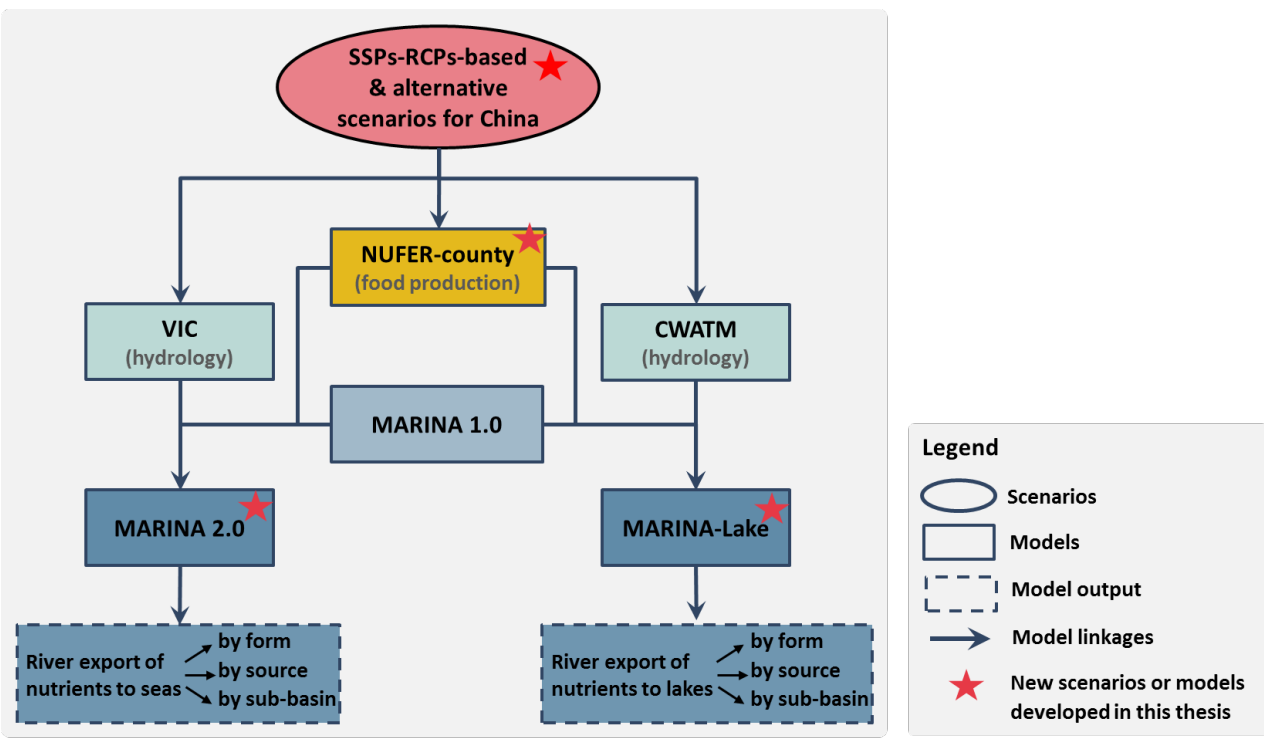

Figure 1.3 The linked model system for modeling river export of nutrients to Chinese seas and lakes developed in this thesis. SSPs are Shared Socioeconomic Pathways (O'Neill et al., 2014. RCPs are Representative Concentration Pathways (Van Vuuren et al., 2011). VIC is the Variable Infiltration Capacity model (Liang et al., 1994; Van Vliet et al., 2016a). CWATM is the Community WATer Model (Burek et al., 2017). NUFER is the NUtrient flows in Food chains, Environment and Resources use model (Ma et al., 2010; Wang et al., 2018b). MARINA 1.0 (or 2.0) is the Model to Assess River Inputs of Nutrient to seAs (Strokal et al., 2016a; Wang et al., Under Review). MARINALake is the Model to Assess River Inputs of Nutrients to lAkes (Wang et al., 2019a).

\subsection{Thesis outline}

This thesis consists of eight chapters. Each research sub-objective forms a scientific paper, presented in Chapters 2-7. Figure 1.2 shows an overview of all chapters with their objectives and methods. Figure 1.4 shows the study area and scales of research presented in Chapters 2-7.

In Chapter 2, I identified the hotspots for $\mathrm{N}$ and $\mathrm{P}$ losses to the environment from food production in China (sub-objective 1). I developed a new NUFER-county model and applied this model in this chapter. $\mathrm{N}$ and $\mathrm{P}$ losses from food production to the environment for all counties (>2500 counties) in China were quantified for 1990, 2000 and 2012. 
In Chapter 3, I analyzed the sources of river export of nutrients to Lake Taihu and compared these with the critical nutrient loads of the lake (sub-objective 2). I developed a MARINA-Lake model and applied the model for Lake Taihu in this chapter. I studied a drainage area of the Taihu basin covering $15,723 \mathrm{~km}^{2}$. The basin is divided into five subbasins (naming I-V) including two upstream and three downstream sub-basins.

In Chapter 4, I analyzed future reactive $\mathrm{N}$ (Nr) losses from China's food system as affected by socio-economic development, taking the storylines of five SSPs as a starting point (subobjective 3). Therefore, I applied the NUFER-national model to quantify Nr losses from China's food system. This is the first study to quantify future Nr losses from China's food system for the SSPs.

In Chapter 5, I analyzed how socio-economic and climate change together will affect coastal eutrophication through future river export of total dissolved nitrogen and phosphorus by Chinese rivers (sub-objective 4). To this end, I developed the new MARINA 2.0 model and applied the model in this Chapter. I developed six scenarios combing the changes in socio-economic (SSPs) and climate (RCPs) changes and implemented these in MARINA 2.0. I studied 12 Chinese rivers draining into the Bohai Gulf, Yellow Sea, East China Sea, and the South China Sea. The drainage area of the rivers covers $40 \%$ of China's land. The drainage area of three large rivers: the Yellow, Yangtze, and Pearl rivers are divided into upstream, middle-stream and downstream sub-basins.

In Chapter 6, I analyzed how current and improved nutrient management can affect future nutrient use efficiencies and nutrient losses from food production (sub-objective 5). I did this by applying the NUFER-provincial model to all provinces in China. This is the first study to analyze the effectiveness of the current "Zero Growth in Synthetic Fertilizer Use from 2020" policy (MOA, 2015c) on improving nutrient use efficiencies in food production in China.

In Chapter 7, I explored future scenarios to reduce nutrient pollution in rivers and coastal waters of China, and to contribute to the achievement of SDGs 6 and 14 (sub-objective 6). Based on the MARINA 2.0 model, I studied six large Chinese rivers: Liao, Hai, Huai, Yellow, Yangtze and Pearl rivers. The drainage area of three large rivers: the Yellow, Yangtze, and Pearl rivers are divided into upstream, middle-stream and downstream sub-basins.

Lastly, in Chapter 8, I discussed the methods and results and drew the main conclusions of this thesis. 

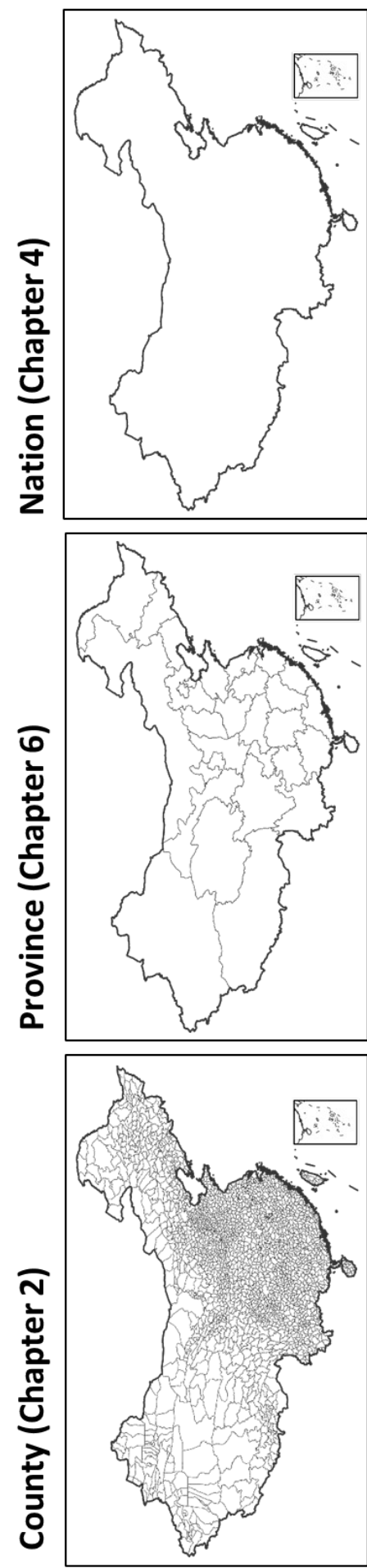

$\Xi \Xi$

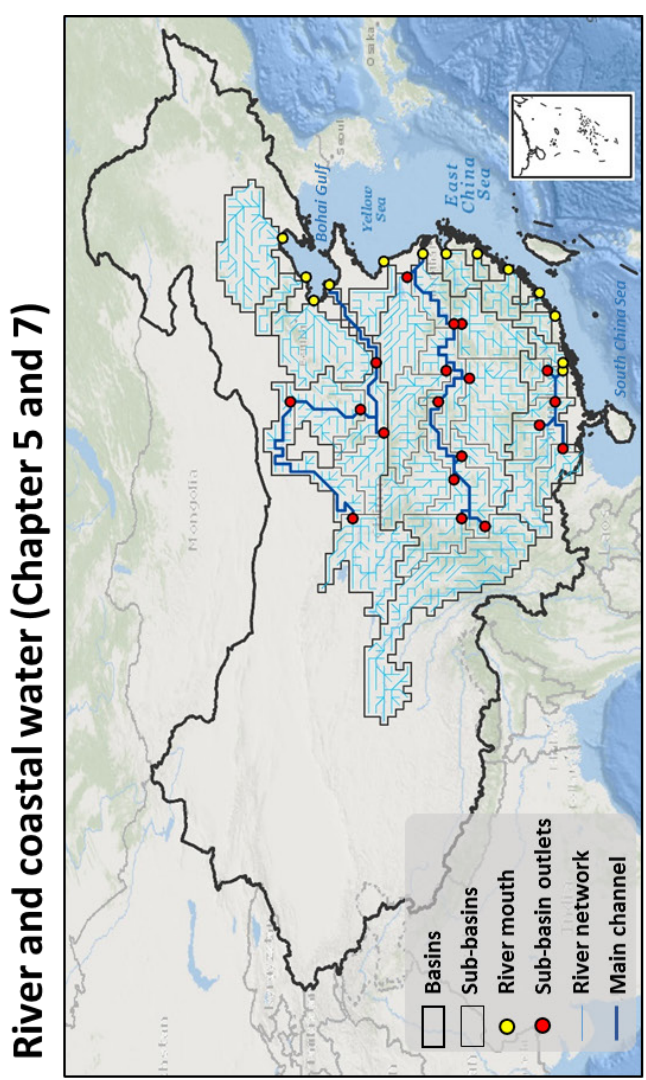

ㄱำ

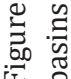

I

ฮั.

巳 돞

.

을 ฮี

¿

巳

苂

Ð

范

\&

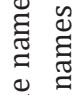

E

ت

\&

㐫

ए

里

두

异:

零

¿

कิ

离

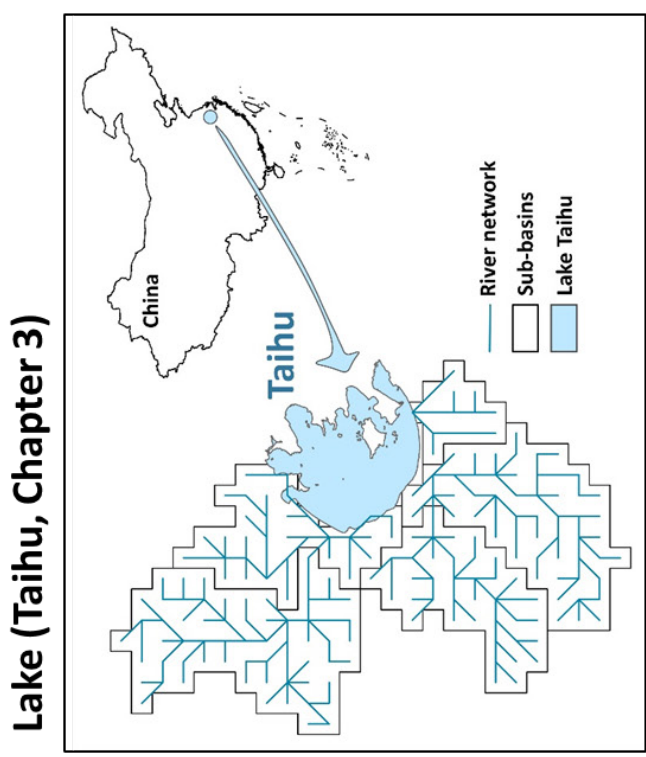

记

돞

त

ขึ थै

๘

골 胥

吾苞

¿

¿ ธิ

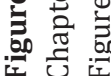





\title{
Chapter 2. Hotspots for Nitrogen and Phosphorus Losses from Food Production in China: A County-Scale Analysis
}

\begin{abstract}
Food production in China results in large losses of nitrogen $(\mathrm{N})$ and phosphorus (P) to the environment. Our objective is to identify hotspots for $\mathrm{N}$ and $\mathrm{P}$ losses to the environment from food production in China at the county scale. To do this, we used the NUFER (NUtrient flows in Food chains, Environment and Resources use) model. Between 1990 and 2012, the hotspot area expanded by a factor of 3 for N, and 24 for P. In 2012 most hotspots were found in the North China Plain. Hotspots covered less than $10 \%$ of the Chinese land area, but contributed by more than half to $\mathrm{N}$ and $\mathrm{P}$ losses to the environment. Direct discharge of animal manure to rivers was an important cause of $\mathrm{N}$ and P losses. Food production was found to be more intensive in hotspots than in other counties. Synthetic fertilizer use and animal numbers in hotspots were a factor of 4-5 higher than in other counties in 2012. Also the number of people working in food production and the incomes of farmers are higher in hotspots than in other counties. This study concludes with suggestions for region-specific pollution control technologies for food production in China.
\end{abstract}

Published as:

Wang M., Ma L., Strokal M., Ma W., Liu X., Kroeze C. Hotspots for Nitrogen and Phosphorus Losses from Food Production in China: A County-Scale Analysis. Environmental Science \& Technology 2018; 52: 5782-5791. https://pubs.acs.org/doi/10.1021/acs.est.7b06138. Further permissions related to the material excerpted should be directed to the ACS. 


\subsection{Introduction}

Nitrogen (N) and phosphorus (P) applications in food production greatly contribute to global food security as essential nutrients for the growth of plants and animals. However, this can also lead to N and P losses to the environment (Bouwman et al., 2013; Conway and Pretty, 2013; Mosier et al., 2013; Reay et al., 2012; Tilman et al., 2011; Zhang et al., 2013). Increasing losses of $N$ and $P$ to surface waters and the atmosphere have negative impacts on ecosystems and biodiversity. For example, increased $\mathrm{N}$ and $\mathrm{P}$ losses from food production may cause eutrophication and harmful algal blooms in many coastal areas around the world (Dumont et al., 2005; Seitzinger et al., 2010; Strokal et al., 2014b; Strokal et al., 2014c; Yasin et al., 2010).

$\mathrm{N}$ and $\mathrm{P}$ losses from food production including crop and animal production in China have been increasing since the 1980s (Hartmann et al., 2015; Li et al., 2015b; Lu et al., 2015; Ma et al., 2013b). Ma et al. (2012b) estimated that total P losses from crop production in 2005 were $300 \%$ higher than that in 1980, while from animal production P losses were more than 42 times as high as that in 1980 in China. This implies that an increasing amount of nutrients was lost to air and water (Li et al., 2007; Ma et al., 2008; Qu and Kroeze, 2010; Strokal et al., 2014b; Wang et al., 2011a; Zhang et al., 2013). Various studies discussed the reasons why nutrient losses in China are high and increasing (Chen et al., 2008; Hou et al., 2013; Qu and Kroeze, 2010; Strokal et al., 2014b; Wang et al., 2010a; Wang et al., 2011a; Zhang et al., 2013; Zhu and Jin, 2013). An important reason is poor nutrient management technologies in food production: overuse of synthetic fertilizers and animal feeds, low productivity, and poor management of animal manure. However, most existing analyses are limited to the national or provincial levels, and they often do not discuss the possible relations between nutrient losses and socio-economic conditions that are usually the drivers of intensive food production in China (Bai et al., 2014b; Chen et al., 2008; Hou et al., 2013; Liu et al., 2004; Wang et al., 2010a). A more detailed analysis of $\mathrm{N}$ and $\mathrm{P}$ losses from food production at, for instance, the county scale does not exist. Such an analysis is highly relevant because it can provide more explicit quantitative information on $\mathrm{N}$ and $\mathrm{P}$ flows in food production in China. The result will contribute to identifying the 'hotspots' where $\mathrm{N}$ and $\mathrm{P}$ losses from food production are higher than other regions. Analyzing the $\mathrm{N}$ and $\mathrm{P}$ flows in the hotspots and local agricultural and socio- 
economic indicators may help to develop region-specific nutrient management technologies and policies to reduce the potential for nutrient pollution in China.

Thus, our study aims to identify hotspots for $\mathrm{N}$ and $\mathrm{P}$ losses to the environment from food production in China at the county scale. To do this, we used the NUFER (NUtrient flows in Food chains, Environment and Resources use) model (Ma et al., 2010). We analyzed N and P losses from food production including crop and animal production for all Chinese counties in 1990, 2000, and 2012. We also quantified the associated N and P use efficiencies of food production in China to better understand the high losses in the hotspots. We compared several agricultural and socio-economic indicators for the hotspots with that for other counties in 2012. One the basis of the results, we conclude with suggestions on technologies and policies for nutrient pollution control in food production of China.

\subsection{Materials and Methods}

\subsubsection{NUFER Model}

In this study, we used the NUFER (Ma et al., 2010; Ma et al., 2012b) model to quantify N and P losses from food production for all counties in China for 1990, 2000, and 2012. Food production includes crop and animal production in this study (Figure S1 in Appendix I). Years 1990, 2000, and 2012 were selected to reflect the period during which the transition of food production in agriculture from traditional small scale to intensive large scale took place in China (see Section S1 in Appendix I for more information about the transition). The original NUFER model was developed by Ma et al. (2010) to quantify N and $\mathrm{P}$ flows in the food production, processing and consumption chain of China using a mass balance approach. This model calculates nutrient flows at the national level for each year from 1980 to 2010, and for the year 2030. In addition, regional nutrient flows can be calculated for 31 provinces for 2005 and 2013. The detailed model description on the original NUFER model is available in Section S2 in Appendix I. We developed and applied NUFER for all Chinese counties using county information (RESDC, 2013), which was not done before. We also improved NUFER by including dry atmospheric $\mathrm{N}$ deposition on arable land that was not included in the original model. We described in Section S2 in Appendix I how we included dry atmospheric $\mathrm{N}$ deposition and applied NUFER to the county scale. 


\subsubsection{Identifying Hotspots}

We quantified the $\mathrm{N}$ and $\mathrm{P}$ losses to waters and the air from food production including crop and animal production systems using NUFER. The losses to waters are the $\mathrm{N}$ and $\mathrm{P}$ losses from leaching, surface runoff, and erosion in crop production, and $\mathrm{N}$ and $\mathrm{P}$ losses from direct discharge of animal manure in animal production. For the losses to the air, we calculated the emissions of ammonia $\left(\mathrm{NH}_{3}\right)$ and nitrous oxide $\left(\mathrm{N}_{2} \mathrm{O}\right)$ from crop and animal production systems. The details in the calculation of $\mathrm{N}$ and $\mathrm{P}$ losses using NUFER are available in Section S2 in Appendix I. The calculated $\mathrm{N}$ and P losses in all counties were averaged by the area of the counties and were grouped into four groups (Figures 2.1 and 2.2). The intervals for the four groups were defined based on quantiles ( $25 \%, 50 \%, 75 \%)$ of the averaged $\mathrm{N}$ and P losses of all counties in 2012: group I (0-25\%), group II (25-50\%), group III (50-75\%), group IV (75-100\%, hotspot). The top 25\% were considered hotspots. For 1990, 2000 and 2012 counties were considered as hotspots if their $\mathrm{N}$ and P losses fall within the range of the top $25 \%$ for 2012 . Thus for all three years counties that have total $\mathrm{N}$ losses exceeding $9625 \mathrm{~kg} \mathrm{~N} \mathrm{~km}^{-2}$ year-1 were qualified as hotspots (Figure 2.1, and Figure S2 in Appendix I). For P, counties with total losses of more than $905 \mathrm{~kg} \mathrm{P} \mathrm{km}^{-2}$ year${ }^{1}$ were qualified as hotspots (Figure 2.2, and Figure S3 in Appendix I). We did not identify hotspots based on water or air quality standards in China, because these standards differ among regions (e.g., provinces) and the use of the water body. For example, the water quality standards for drinking water supply differ from standards for water that are used for agricultural purposes. Therefore, we used the top 25\% for 2012 as a basis for the identification of hotspots as shown in Figures 2.1 and 2.2. We also calculated the associated $\mathrm{N}$ and $\mathrm{P}$ use efficiencies of food production for a better understanding of $\mathrm{N}$ and $\mathrm{P}$ losses. $\mathrm{N}$ and $\mathrm{P}$ use efficiencies were calculated as outputs of $\mathrm{N}$ and $\mathrm{P}$ via main products divided by the total inputs of $\mathrm{N}$ and $\mathrm{P}$ to this system (Box S3 in Appendix I).

We included in our result (Figures 2.1 and 2.2, Figures S2 and S3 in Appendix I) borders of the nine Agro-Ecological Zones (AEZs) in China to illustrate the spatial distribution of the hotspots for $\mathrm{N}$ and $\mathrm{P}$ losses. The AEZs are Northeast China, Inner Mongolia and Great Wall Vicinity, North China Plain, Loess Plateau, Middle and Lower Yangtze River, Southwest China, South China, Gansu and Xinjiang, and Tibetan Plateau (see Figure S8 in Appendix I for the location of the AEZs). These zones were defined by Sun and Shen (1994) based on their similarities in crop production (e.g. cropland, crop types) and animal production (e.g. animal type, animal numbers) systems. 


\subsubsection{Comparing Agricultural and Socio-economic Indicators in Hotspots with Nonhotspots}

We compared several agricultural and socio-economic indicators in hotspots (group IV in Figures 2.1 and 2.2, and in Figures S4 and S5 in Appendix I) and nonhotspot counties (groups I, II, III in Figures 2.1 and 2.2, and in Figures S4 and S5 in Appendix I) in 1990 and 2012. The agricultural indicators are (i) $\mathrm{N}$ and $\mathrm{P}$ inputs to cropland from synthetic fertilizer, (ii) animal numbers, (iii) share of sown area of vegetable and fruit to total sown area, (iv) $\mathrm{N}$ and $\mathrm{P}$ use efficiencies of food production including crop and animal production. The socio-economic indicators are (i) urban population, (ii) rural labor, (iii) total output value of agriculture and forestry, and (iv) farmers' incomes. We selected all socioeconomic indicators that are available in county statistics. The Tukey's Honest Significant Difference (Tukey's HSD) method was used to make pairwise comparisons of the agricultural and socio-economic indicators among the groups I, II, III, and IV that was defined above. The results of Tukey's HSD comparison are shown in Figures 2.3 and 2.4, and in Figures S4 and S5 in Appendix I.

\subsection{Results}

\subsubsection{Hotspots for $\mathrm{N}$ and $\mathrm{P}$ Losses}

The N and P losses increased fast between 1990 and 2012. As a result, the hotspot area in China expanded during this period. The hotspot area for total $\mathrm{N}$ losses increased by a factor of 3 (from 307,850 to 828,205 $\mathrm{km}^{2}$ ), and for total P by a factor of 24 (from 35,355 to $861,781 \mathrm{~km}^{2}$ ) between 1990 and 2012 (Table S6 in Appendix I). The hotspots covered less than $5 \%$ of total land and contributed to $28 \%$ of total $\mathrm{N}$ losses, and $10 \%$ of total $\mathrm{P}$ losses in food production of China in 1990. In 2012, the hotspot area for total $\mathrm{N}$ and total $\mathrm{P}$ losses expanded to $9 \%$ of the total land in China. Astoundingly, these hotspots contributed more than half of nutrient losses (52\% of total $\mathrm{N}$ losses, and $62 \%$ of total $\mathrm{P}$ losses) in this year. The increase in hotspot area and in contributions to total losses in China by hotspots are also calculated for $\mathrm{N}$ and $\mathrm{P}$ losses from various sources in Figures 2.1 and 2.2 and Table S6 in Appendix I.

The spatial distribution of hotspots also changed between 1990 and 2012. In 1990, most hotspots are found in the North China Plain, and in the northeastern part of the Middle and Lower Yangtze River (Figures 2.1 and 2.2). In 2012, the hotspots expanded to cover a larger area of the North China Plain. Some counties in the AEZs Middle and Lower Yangtze 
River, Northeast China, Loess Plateau, Southwest China, and South China also show high $\mathrm{N}$ and P losses as hotspots. In the AEZs where the hotspot areas expanded, the food production is intensive and increased fast between 1990 and 2012 (Figure S9 in Appendix I). The nonhotspots with low $\mathrm{N}$ and P losses (groups I and II in Figures 2.1 and 2.2) are found across northern and western China in 2012, for example, in AEZs Inner Mongolia and Great Wall Vicinity, Gansu and Xinjiang, and Tibetan Plateau where food production is less intensive (Figure S9 in Appendix I).

The result also shows that direct discharge of animal manure to waters in the hotspots became a more important source of $\mathrm{N}$ and $\mathrm{P}$ losses in food production over the last 30 years. In 1990, the hotspots for $\mathrm{N}$ and $\mathrm{P}$ losses to waters from direct discharge of manure only covered $0.2 \%$ and $0.3 \%$ of the total land. These hotspots were responsible for less than $10 \%$ of the total $\mathrm{N}$ and P losses from discharge of manure (Table S6 in Appendix I). However, in 2012 the hotspots area for direct discharge of manure is calculated to increase by a factor of 51 for $\mathrm{N}$ losses and of 33 for P losses comparing to 1990 . And these hotspots contributed to more than half of $\mathrm{N}$ and $\mathrm{P}$ losses (57\% for $\mathrm{N}$ and $64 \%$ for $\mathrm{P}$ ) from direct discharge of manure in China (Table S6 in Appendix I). This change in N and P losses from direct discharge of animal manure could be related to the transition of food production in China started in 1990s (Chadwick et al., 2015; Strokal et al., 2016a) (Section S1, Figures S6 and S7 in Appendix I). Traditional-oriented food production was dominant in the 1990s. Traditional animal systems are small in size and combined with crop production. Animal manure is usually used as organic fertilizer for crops. Therefore, the discharge of manure to waters is relatively low (Bai et al., 2013; Schneider, 2011; Wang, 2007). Industrial, highly intensive systems dominate food production in China since 2000s. Industrial animal production systems are large in size and are separated from crop production. The animal manure is usually collected, and discharged to surface waters or landfills without treatment (Bai et al., 2014b; Bai et al., 2013; Chadwick et al., 2015; Schneider, 2011; Wang, 2007). Therefore, the discharge of manure to waters has been increasing since 2000 particularly in the counties that have intensive animal production activities. Our results in Figures S6 and S7 in Appendix I indicate that intensive animal production in the hotspots is considerably higher than that in nonhotspot counties $(\mathrm{P}<0.05)$. This situation lasted at least until the "Regulation on the Prevention and Control of Pollution from Large-Scale Breeding of Livestock and Poultry" was introduced by Chinese Premier Li (2013), and implemented since 1 January 2014. By introducing this 
policy, the Chinese government aims to improve manure management and manure recycling in order to reduce the environmental pollution caused by intensive animal production. The "Livestock and Poultry Manure Utilization Action Program (2017- 2020)" was introduced by MOA (2017) in late 2017. As a result, direct discharge of animal manure may become smaller in the future. Our results present the situation in 2012, when policies on manure management were not widely introduced and not very effective. 

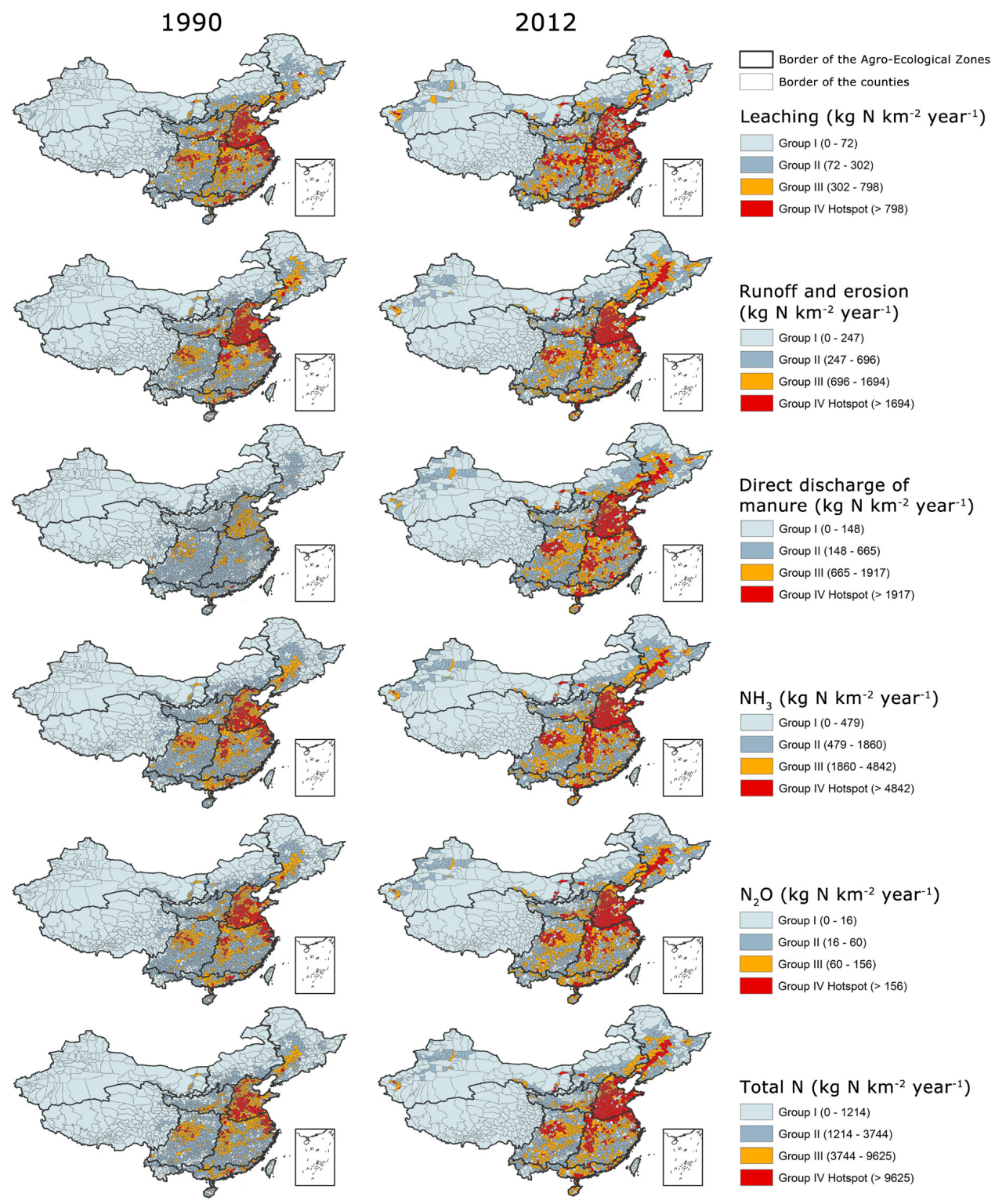

Total $\mathrm{N}\left(\mathrm{kg} \mathrm{N} \mathrm{km}^{-2}\right.$ year $\left.^{-1}\right)$

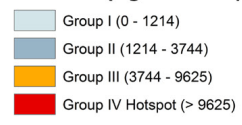

Figure 2.1 Nitrogen ( $\mathrm{N})$ losses $\left(\mathrm{kg} \mathrm{N} \mathrm{km}^{-2}\right.$ year-1) $^{-1}$ to the air and waters from leaching, runoff and erosion, direct discharge of manure, ammonia $\left(\mathrm{NH}_{3}\right)$ and nitrous oxide $\left(\mathrm{N}_{2} \mathrm{O}\right)$ emissions, and the total $\mathrm{N}$ losses $\left(\mathrm{kg} \mathrm{N} \mathrm{km}^{-2}\right.$ year-1) from food production in 1990 and 2012. The $\mathrm{N}$ losses were quantified using the NUFER (NUtrient flows in Food chains, Environment and Resources use) model. The intervals for the four groups in this figure were defined based on quantiles $(25 \%, 50 \%$, $75 \%$ ) of $\mathrm{N}$ losses of all counties in 2012: group I (0-25\%), group II (25-50\%), group III (50-75\%), group IV (75-100\%). Counties in group IV were qualified as hotspots. The same information for 2000 is available in Figure S2 in Appendix I. The names of the Agro-Ecological Zones are available in Figure S8 in Appendix I. 
1990
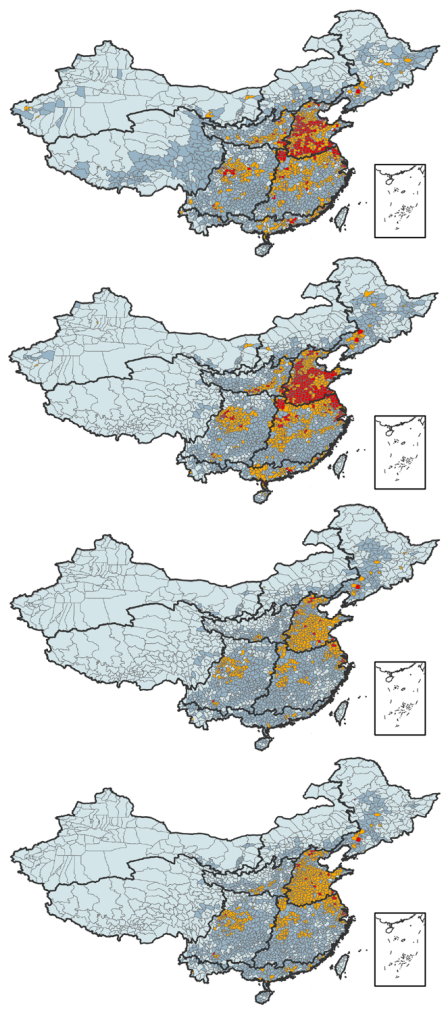

2012
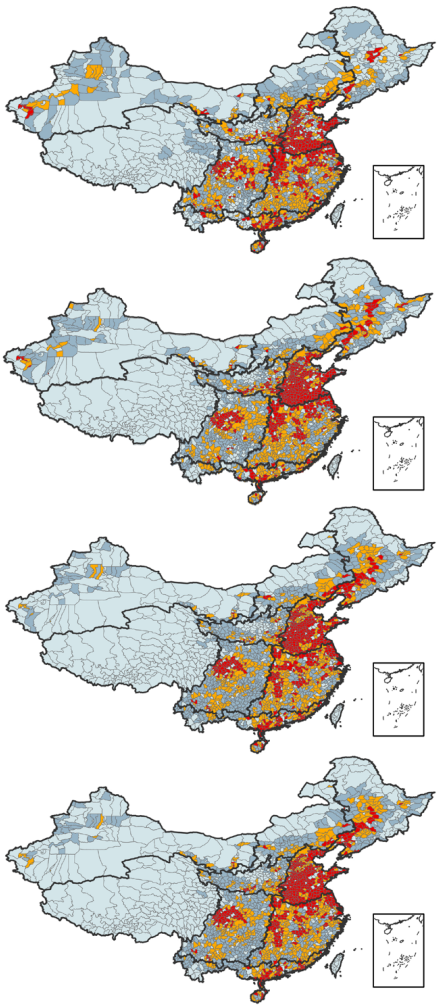

Border of the Agro-Ecological Zones

Border of the counties

Leaching $\left(\mathrm{kg} \mathrm{P} \mathrm{km}^{-2}\right.$ year $\left.^{-1}\right)$

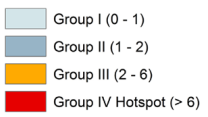

Runoff and erosion

$\left(\mathrm{kg} \mathrm{P} \mathrm{km}^{-2}\right.$ year $\left.^{-1}\right)$

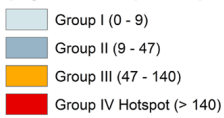

Direct discharge of manure $\left(\mathrm{kg} \mathrm{P} \mathrm{km}^{-2}\right.$ year $\left.^{-1}\right)$

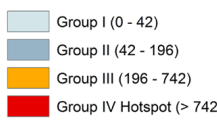

Total $\mathrm{P}\left(\mathrm{kg} \mathrm{P} \mathrm{km} \mathrm{km}^{-2}\right.$ year $\left.^{-1}\right)$

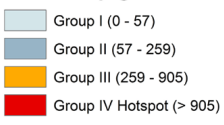

Figure 2.2 Phosphorus (P) losses ( $\mathrm{kg} \mathrm{P} \mathrm{km}^{-2}$ year-1) to waters from leaching, runoff and erosion, direct discharge of manure, and the total $\mathrm{P}$ losses $\left(\mathrm{kg} \mathrm{P} \mathrm{km}^{-2}\right.$ year-1) from food production in 1990 and 2012. The P losses were quantified using the NUFER (NUtrient flows in Food chains, Environment and Resources use) model. The intervals for the four groups in this figure were defined based on quantiles $(25 \%, 50 \%, 75 \%)$ of P losses of all counties in 2012: group I (0-25\%), group II (25-50\%), group III (50-75\%), group IV (75-100\%). Counties in group IV were qualified as hotspots. The same information for 2000 is available in Figure S3 in Appendix I. The names of the Agro-Ecological Zones are available in Figure S8 in Appendix I. 


\subsubsection{Agricultural and Socio-economic Indicators}

Comparing the hotspots with nonhotspot counties shows that food production in the hotspots was more intensive than in other counties (Figures 2.3 and 2.4, Figures S4 and S5 in Appendix I, $\mathrm{P}<0.05$ ) in both past (1990) and recent (2012) years. The mean synthetic fertilizer use and mean animal numbers in the hotspots were much higher than in other counties in 2012 (Figures 2.3 and 2.4, $\mathrm{P}<0.05$ ). For example, the mean synthetic fertilizer input in the hotspots was $400 \%$ higher for $\mathrm{N}$, and $300 \%$ higher for $\mathrm{P}$ than that in other counties (Table S7 in Appendix I). Mean animal numbers in the hotspots were five times that in other counties in 2012 (Table S7 in Appendix I).

The $\mathrm{N}$ and $\mathrm{P}$ use efficiencies of food production are calculated to be low and their means are comparable between hotspots and nonhotspot counties (Figures 2.3 and 2.4, Figures $\mathrm{S} 4$ and S5 in Appendix I, P>0.05). The average nutrient use efficiency of food production in Chinese counties was 24\% for N, and 29\% for P in 2012 (Table S7 in Appendix I). This is lower than the nutrient use efficiency in 1990 that was $25 \%$ for $\mathrm{N}$ and $38 \%$ for $\mathrm{P}$ (Figures S4 and S5 in Appendix I). Howarth et al. (2002) estimated the $\mathrm{N}$ use efficiency of main crop production at $56 \%$ in United States in 2000. And in Europe the $\mathrm{N}$ use efficiency of main crop production was around $44 \%$ in 2000 (Oenema et al., 2009). This indicates that in general the nutrient inputs in food production in China are not used efficiently because of poor nutrient management in food production, and as consequence losses of nutrients to the air and waters are relatively high. However, the fact that nutrient use efficiencies in hotspots do not differ from those in nonhotspots, indicates that low $\mathrm{N}$ and $\mathrm{P}$ use efficiencies are not the only reason for the high losses in the hotspots. The high $\mathrm{N}$ and $\mathrm{P}$ losses are the net effect of low $\mathrm{N}$ and $\mathrm{P}$ use efficiencies, intensive food production (e.g., large animal numbers), and poor nutrient management technologies (e.g., overuse of synthetic fertilizer, and high direct discharge of manure to waters as mentioned above) in the hotspots.

The shares of vegetables and fruits in the total sown area in the hotspots are comparable to that in nonhotspot counties (Figures 2.3 and 2.4, Figures S4 and S5 in Appendix I, $\mathrm{P}>0.05$ ). Production of vegetables and fruit was not found to be more intensive in hotspots than in other counties. This is surprising since earlier studies indicate that nutrient losses from fruit and vegetable production are usually higher than that in other cropping 
systems as a result of high synthetic fertilizer application (Bai et al., 2016; Chen et al., 2018; Heffer et al., 2017).

Socio-economic indicators also differ between hotspots and other counties (Figures 2.3 and 2.4, Figures S4 and S5 in Appendix I, P<0.05). Hotspots are less urbanized. On average less than $20 \%$ of population was urban in the hotspots in 2012 (Table S7 in Appendix I). On the other hand, the mean number of people working in food production in hotspots was three times that in nonhotspot counties in 2012 (Table S7 in Appendix I). The mean incomes for farmers who work in food production were over $30 \%$ higher in the hotspots than that in the nonhotspot counties (Table S7 in Appendix I). Also the mean total output value from agriculture and forestry in these hotspots was considerably higher (close to five times) than in other counties in 2012 (Figures 2.3 and 2.4, $\mathrm{P}<0.05$ ). Therefore, farmers' incomes in the hotspots seem to be more dependent on food production. 

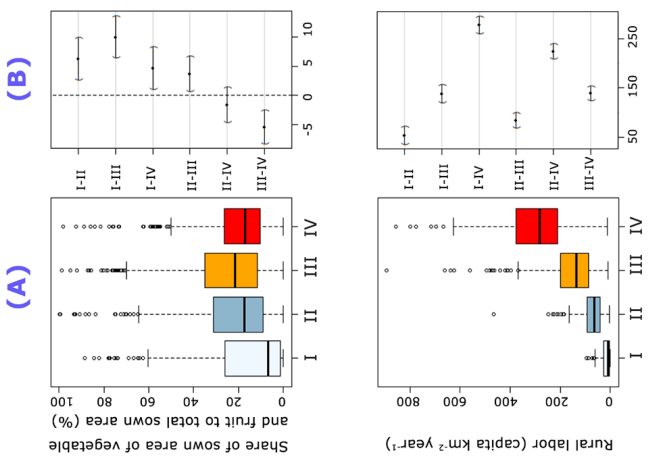

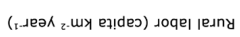
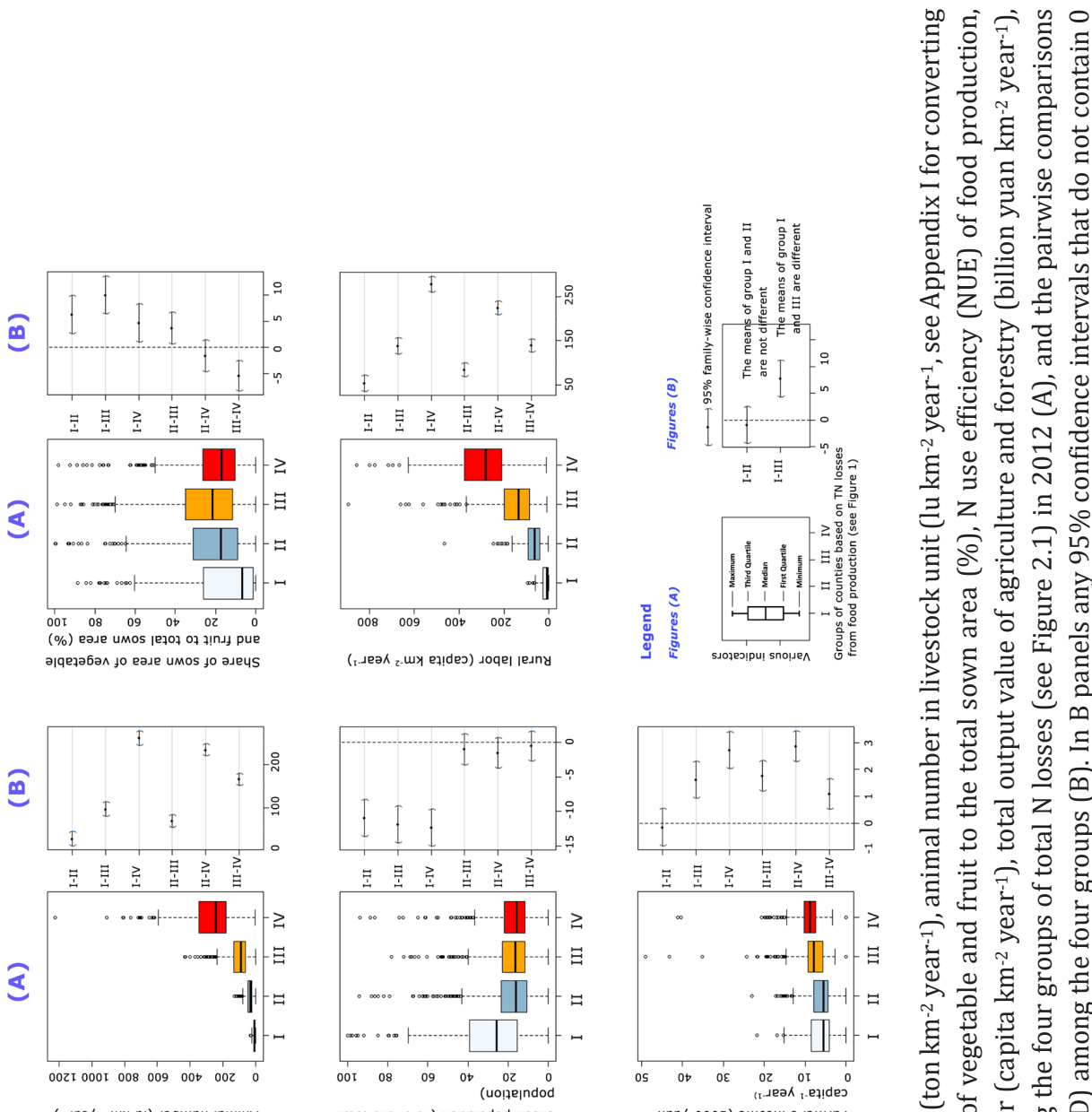

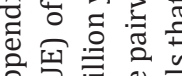

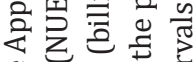
施

ॠ

ฮี तิ ¿ี こ乙

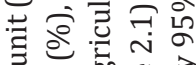

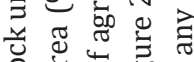
苍 ¿ $\Xi \frac{\pi}{\square}$ थ ذั

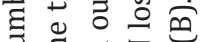
吉艺云的 오ㅇㅠㅠ

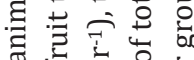
สิ

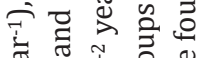

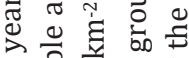

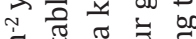

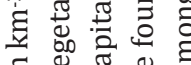
ป

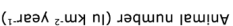

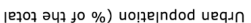
uenk 000i) amozul s.jamis
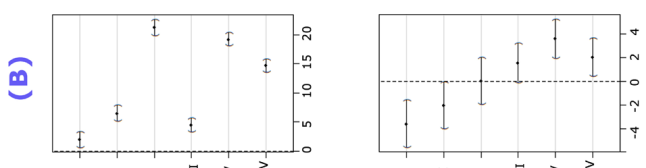

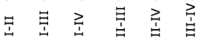
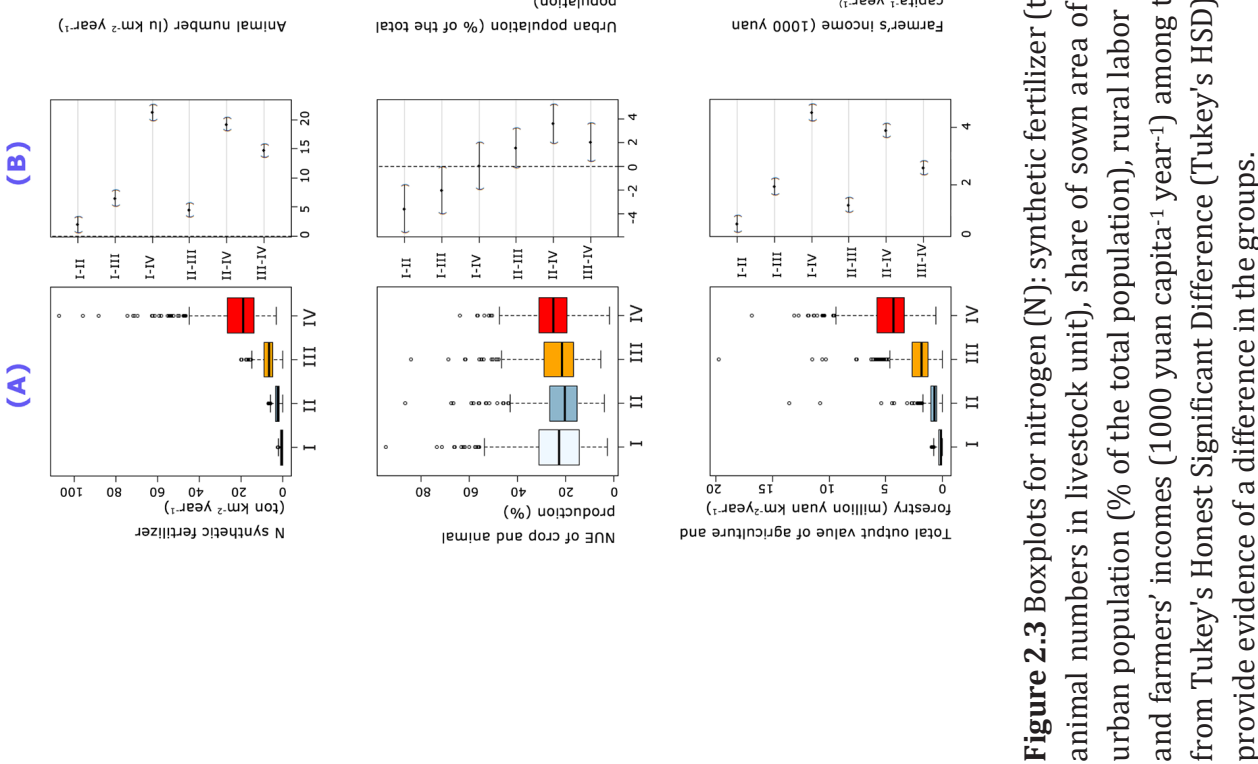

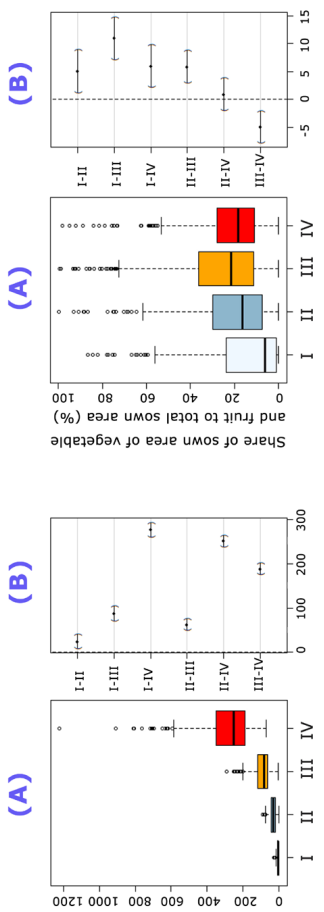

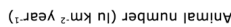
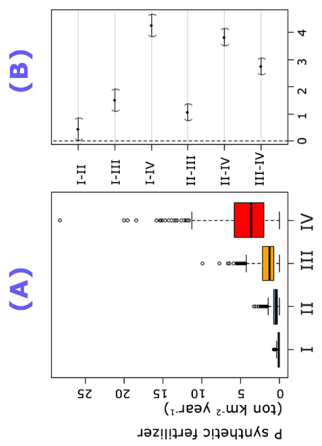

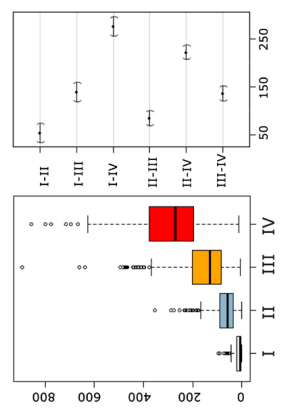

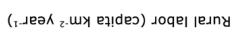
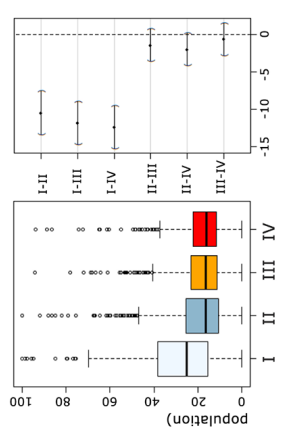

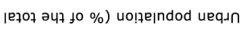
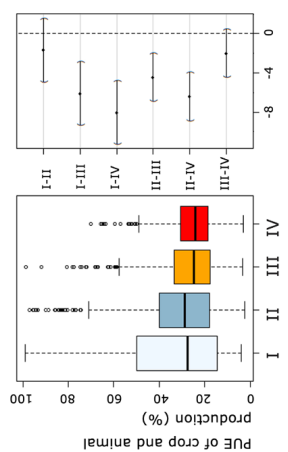
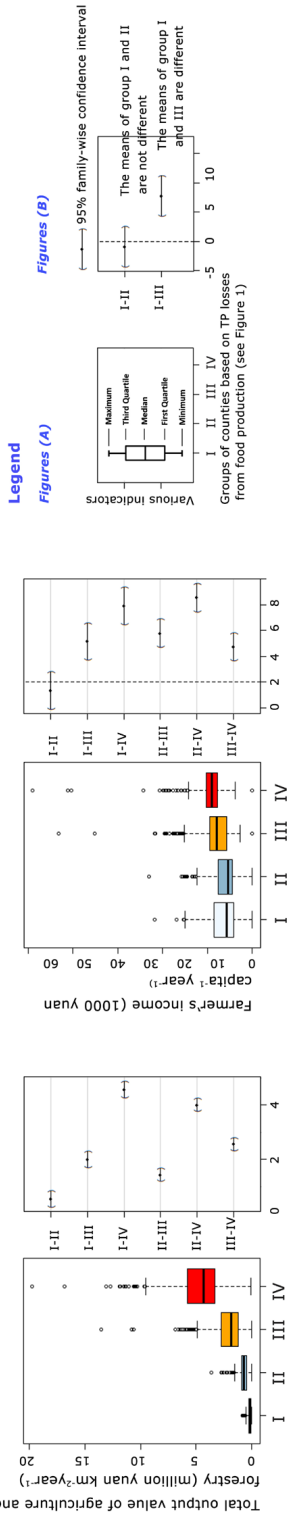

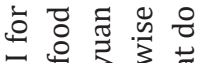

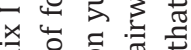

엉월

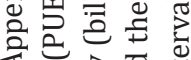

¿ $\overrightarrow{0}$ 范

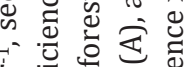

需焉过

ঠ

ก

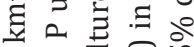

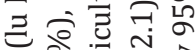

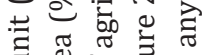

$\Xi$ 㫕范

ป

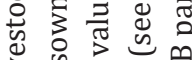

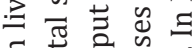

$\Xi$ ث艹

है

워융

䒕节范

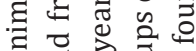

สิ

正实

ฮี

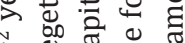

엉

द्व

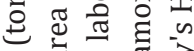

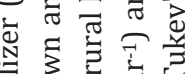

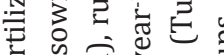

屯

.

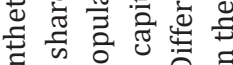

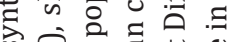

赔志芯芯芯

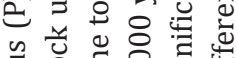

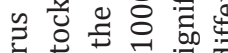

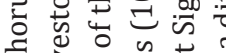

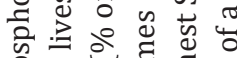

을

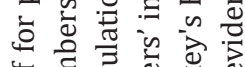

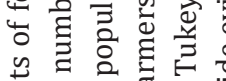

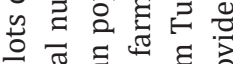

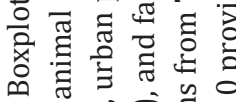

ฟ

ง.

๑

แ్ 


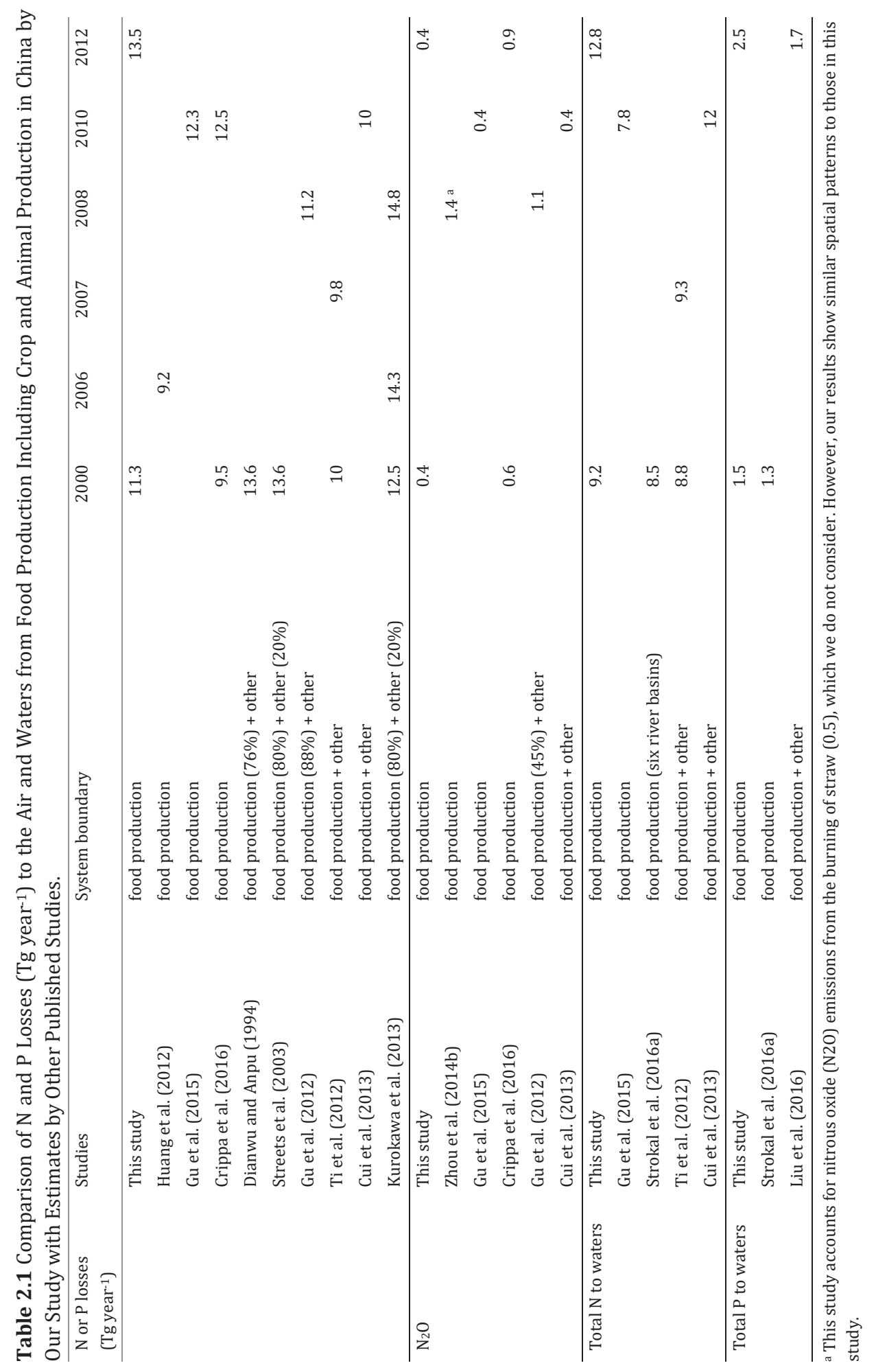




\subsection{Discussion}

This study is the first to calculate past (1990 and 2000) and more recent (2012) N and P losses to the environment from food production in China at the county scale. On this basis we identified the associated hotspots for $\mathrm{N}$ and $\mathrm{P}$ losses from food production. We compared several agricultural and socio-economic indicators for the hotspots with that for other counties in 2012. The main findings are as follows.

We calculate a larger hotspot area for 2012 than for 1990, indicating $\mathrm{N}$ and P losses from food production increased in this period. In 2012, the hotspots covered $9 \%$ of the total land, but were responsible for $52 \%$ of total $\mathrm{N}$ losses and $62 \%$ of total P losses in China. Nutrient losses from food production in the hotspots are higher than $9625 \mathrm{~kg} \mathrm{~km}^{-1}$ for N, and $905 \mathrm{~kg} \mathrm{~km}^{-1}$ for P. Note that these losses are even higher than the recommended fertilizer inputs to arable land. For example, the United Kingdom suggests to apply 120 $270 \mathrm{~kg} \mathrm{ha}^{-1} \mathrm{~N}$ on arable land (GOVUK, 2015). Such high losses to the air and waters pose potentially high risks to the environment. The direct discharge of animal manure has become an important source of $\mathrm{N}$ and $\mathrm{P}$ losses in food production over the last 30 years as the result of industrialization of animal production in China as indicated in Table S9 in Appendix I.

The hotspots expanded from part of North China in 1990 to most of the area of North China Plain, and some areas of other eastern AEZs in 2012. Food production in the hotspots is found to be intensive. Mean synthetic fertilizer use and mean animal numbers in hotspots were 300-400\% higher than that in nonhotspot counties in 2012. N and P use efficiencies of food production were generally low (24\% for NUE, and 29\% for PUE) in 2012 and did not differ much between hotspots and nonhotspot counties. Therefore, low use efficiency of nutrients is not the only factor to explain the high losses of $\mathrm{N}$ and $\mathrm{P}$ in hotspots. The high losses of $\mathrm{N}$ and $\mathrm{P}$ in the hotspots are the net effect of the low $\mathrm{N}$ and $\mathrm{P}$ use efficiencies, and the intensive food production in these counties. Less than $20 \%$ of the population is urban in hotspots in 2012. The mean number of people working in food production in hotspots was three times that in other counties in 2012. The mean total output value from agriculture and forestry in hotspots was considerably higher than that in other counties in 2012. Therefore farmers' incomes in hotspots seem to be more dependent on food production than other counties. 
Uncertainties in our analysis are mainly related to the model inputs and the coefficients that are used in the model calculation. In this study, we used county data from the Chinese statistical yearbook as inputs to NUFER. Some of the model inputs were missing in this data set. For the incomplete information, the provincial data from Chinese statistical yearbook and China livestock yearbook were used as a complement (see Section S2 in Appendix I for more details). These statistical yearbooks are known to be the most reliable data source in China. The model coefficients (e.g., nutrient content in crops, nutrient loss factors) are from the original NUFER model, which was taken from other peer-reviewed papers and interviews of farmers in China (Ma et al., 2010). Our results are comparable with other studies. For example, we estimated comparable $\mathrm{NH}_{3}$ losses from food production to the air, and $\mathrm{N}$ and $\mathrm{P}$ losses to waters at the national scale with many of the other studies (Table 2.1). Our estimates of $\mathrm{N}_{2} \mathrm{O}$ emission in 2012 is lower than in the other studies (Table 2.1). A likely explanation is that $\mathrm{N}_{2} \mathrm{O}$ emission from the burning of straw was not considered in our study. This can be improved in our model. However, we do not think this will lead to large change in our conclusions since $\mathrm{N}_{2} \mathrm{O}$ losses are minor compared to other losses of reactive $\mathrm{N}$ from food production (Figures 2.1 and 2.2). Huang et al. (2015); Kang et al. (2016); Zhang et al. (2017) calculated similar spatial distribution patterns for $\mathrm{NH}_{3}$ emissions as we did. The study of Gu et al. (2012) identified similar spatial distribution of $\mathrm{NH}_{3}$ and $\mathrm{N}_{2} \mathrm{O}$ emissions from food production and other human activities and calculated a considerable increase in these emissions between 1990 and 2010. Similar spatial distribution of $P$ losses from food production and other sectors in 2012 was also identified by Liu et al. (2016).

Opportunities to Reduce $\mathbf{N}$ and $\mathbf{P}$ Losses. Our results show that hotspots contributed to more than half of total $\mathrm{N}$ and total $\mathrm{P}$ losses from food production in China, while covering less than $10 \%$ of the country area in 2012. Thus it is important to reduce the nutrient losses from food production in these hotspots in order to control nutrient pollution in China. Region-specific nutrient management needs to be developed for the hotspots particularly for the North China Plain where N and P losses are relatively high, and the food production activities are more intensive than other regions. By comparing the socioeconomic indicators in hotspots and nonhotspots, we found that farmers' incomes in hotspots are more dependent on food production. Therefore, the production of food in the hotspots needs a transformation in order to avoid the negative effects of pollution on the economies of local societies. 
There are many technical improvements possible to reduce losses of nutrients. In crop production, techniques that help to fertilize crops based on their specific needs could reduce $\mathrm{N}$ and $\mathrm{P}$ inputs to land by up to $20 \%$, and improve the associate nutrient use efficiencies in the hotspots (Ma et al., 2013a; Wang et al., 2018a; Zhang et al., 2012; Zhang et al., 2011). Increasing farm sizes may also decrease the use of synthetic fertilizer in crop production (Ju et al., 2016). Nutrient losses to the air and waters could be reduced by up to $60 \%$ for $\mathrm{N}$, and $85 \%$ for $\mathrm{P}$ without reducing crop yields, needed to meet the increasing food demand in China (Wang et al., 2018a). This also secures the interests of farmers since their incomes are dependent on food production. Low-emission technologies (e.g., inject animal manure into soil) could be adopted to reduce the $\mathrm{NH}_{3}$ and $\mathrm{N}_{2} \mathrm{O}$ emission from applying fertilizers on cropland (Bai et al., 2014b; Erisman et al., 2008; Hou et al., 2015). Experimental and modeling studies (Meng et al., 2012; Qin, 2015; Zhang et al., 2012; Zhang et al., 2011) that explore and implement the abovementioned techniques in the North China Plain could be used as a good basis for the nutrient management in hotspots. The intensive industrial animal production leads to large $\mathrm{N}$ and $\mathrm{P}$ losses to the environment in the hotspots. Technologies that improve the quality of animal feed could reduce $20-30 \%$ of the $\mathrm{N}$ and P excretion (Oenema et al., 2009; Wang, 2005a; Wang, 2005b), thus reduce the overall $\mathrm{N}$ and $\mathrm{P}$ losses from animal production. The $\mathrm{N}$ and $\mathrm{P}$ losses to waters from direct discharge of manure could be much reduced by up to $85 \%$ via recycling animal manure on cropland as organic fertilizers and by improving the treatment of animal manure before discharging (Strokal et al., 2017; Wang et al., 2018a). The N and P losses to the air from animal production can be reduced in several ways, for instance by shifting to low emission housing and manure storage technologies that are currently used in some European Union (EU) countries (Velthof et al., 2009). The above nutrient management options are technically effective in reducing $\mathrm{N}$ and $\mathrm{P}$ losses. In future analyses, it is important to also explore whether these options are economically affordable, because the implementation of these options may be a challenge in China.

In summary, our study can be used to identify hotspot counties where pollution control technologies are needed to reduce $\mathrm{N}$ and $\mathrm{P}$ losses from food production. This holds in particular for technologies to reduce synthetic fertilizer use, to improve nutrient use efficiencies of food production, to reduce emissions of $\mathrm{N}$ in animal housing and manure storage, and to increase recycling of manure on land. The challenge will be to secure food production so that farmers' interests are not negatively affected by pollution control. 


\section{Acknowledgments}

This research is sponsored by National Key Research and Development Program of China (2016YFD0800106), Wageningen Institute for Environment and Climate Research (WIMEK) of Wageningen University \& Research, Chinese National Basic Research Program (2015CB150405), the National Natural Science Foundation of China (31572210), President's International Fellowship Initiative, PIFI of the Chinese Academy of Science (2015VEA025), the Hundred Talent Program of the Chinese Academy of Science, the Distinguished Young Scientists Project of Natural Science Foundation of Hebei (D2017503023) for supporting this research. We would like to thank Dr. Zhaohai Bai for his constructive comments and suggestions. Thank to Dr. Zhanqing Zhao for her advice in programming to process the county information. 



\title{
Chapter 3. Excess Nutrient Loads to Lake Taihu: Opportunities for Nutrient Reduction
}

\begin{abstract}
Intensive agriculture and rapid urbanization have increased nutrient inputs to Lake Taihu in recent decades. This resulted in eutrophication. We aim to better understand the sources of river export of total dissolved nitrogen (TDN) and phosphorus (TDP) to Lake Taihu in relation to critical nutrient loads. We implemented the MARINA-Lake (Model to Assess River Inputs of Nutrients to seAs) model for Lake Taihu. The MARINA-Lake model quantifies river export of dissolved inorganic and organic $\mathrm{N}$ and $\mathrm{P}$ to the lake by source from sub-basins. Results from the PCLake model are used to identify to what extent river export of nutrients exceeds critical loads. We calculate that rivers exported $61 \mathrm{kton}$ of TDN and 2 kton of TDP to Lake Taihu in 2012. More than half of these nutrients were from human activities (e.g., agriculture, urbanization) in Sub-basins I (north) and IV (south). Most of the nutrients were in dissolved inorganic forms. Diffuse sources contributed $90 \%$ to river export of TDN with a relatively large share of synthetic fertilizers. Point sources contributed $52 \%$ to river export of TDP with a relatively large share of sewage systems. The relative shares of diffuse and point sources varied greatly among nutrient forms and sub-basins. To meet critical loads, river export of TDN and TDP needs to be reduced by 46-92\%, depending on the desired level of chlorophyll-a. There are different opportunities to meet the critical loads. Reducing $\mathrm{N}$ inputs from synthetic fertilizers and P from sewage systems may be sufficient to meet the least strict critical loads. A combination of reductions in diffuse and point sources is needed to meet the most strict critical loads. Combining improved nutrient use efficiencies and the best available technologies in wastewater treatment may be an effective opportunity. Our study can support the formulation of effective solutions for lake restoration.
\end{abstract}

Published as:

Wang M., Strokal M., Burek P., Kroeze C., Ma L., Janssen A.B.G. Excess nutrient loads to Lake Taihu: Opportunities for nutrient reduction. Science of The Total Environment 2019; 664: 865-873. 


\subsection{Introduction}

Lakes are important sources of freshwater for human needs in China. However, many Chinese lakes are eutrophied, as a result of over-enrichment of nutrients such as nitrogen (N) and phosphorus (P). Environmental problems caused by eutrophication are among the major concerns for future sustainable development in China (Le et al., 2010). A fast growing economy, rapid urbanization and increasing demand for meat production have resulted in increasing amounts of nutrients in Chinese rivers (Gu et al., 2015; Ma et al., 2012b; Strokal et al., 2016b; Wang et al., 2018b). Rivers may export nutrients to lakes. Too much nutrients may deteriorate water quality and promote toxic algal blooms. Toxic algal blooms often lead to disrupted drinking water supply, health issues and odor nuisance for people living in the vicinity of the lake. As a result, the economic costs of algal blooms are estimated at millions of dollars each occurrence (Glibert et al., 2005; Le et al., 2010).

Lake Taihu (south-east China) is one of the Chinese lakes suffering from eutrophication (Qin et al., 2007a). Lake Taihu is located in an area of strong socio-economic development (Le et al., 2010) (see also Section 3.2.1). Algal blooms in Lake Taihu have been reported since 1987 (Duan et al., 2009). Since then, the situation of eutrophication in Lake Taihu has worsened (Janssen et al., 2014). Wuxi's city is located in the Taihu basin. This city depends on Lake Taihu as the major drinking water source. In 2007, the algal blooms in Lake Taihu were so severe that Wuxi's city had to shut down its drinking water station. As a result, many people had to find other water resources for up to a month (Qin et al., 2010).

Many attempts have been made to reduce the eutrophication in Lake Taihu. Examples are the restoration of wetlands (Sun et al., 2015b), mechanical removal of algae (Chen et al., 2012) and flushing with Yangtze water (Hu et al., 2008; Li et al., 2013). So far, these attempts have had limited effects. A possible reason for this is poor understanding of river export of $\mathrm{N}$ and $\mathrm{P}$ to the lake and their sources. Reducing external nutrient loads is a precondition for lowering Lake Taihu's algal growth sufficiently (Janssen et al., 2017; Xu et al., 2015a). It was estimated that most of the nutrients in Lake Taihu were delivered by rivers draining into the lake, whereas only a minor part came from atmospheric deposition and sediment release (Xu et al., 2015a). However, the relative shares of sources in these nutrient inputs are not known. A better understanding of the underlying causes 
of nutrient export by rivers to the lake is needed to formulate effective nutrient management options to reduce eutrophication in Lake Taihu.

A number of modeling studies exist on nutrient inputs to Lake Taihu from different sources (e.g., Huang et al., 2018; Huang et al., 2017; Lai et al., 2006; Liu et al., 2013a). However, these studies often focus on particular areas (e.g., lowland polders), and on total $\mathrm{N}$ and $\mathrm{P}$ loads to the lake. Modeling studies that distinguish between nutrient forms (dissolved inorganic and organic) are lacking. However, distinguishing different nutrient forms is important for effective policymaking because different nutrient forms may have different sources. For example, synthetic (mineral) fertilizers are often the main sources of dissolved inorganic $\mathrm{N}$ and $\mathrm{P}$ in rivers, whereas animal manure and sewage are often the main sources of dissolved organic N and P (Strokal et al., 2016a). Effective management of these different causes of eutrophication requires ability to differentiate between nutrient forms. For each nutrient form the loads can be compared with critical nutrient loads, which are the levels above which we may expect eutrophication problems (Janse et al., 2008). The difference between critical nutrient loads and the actual river export indicates the gap that needs to be closed to ensure a good ecological status of the lake. Moreover, combining this with the information on the sources and forms of $\mathrm{N}$ and $\mathrm{P}$ in the lake will largely contribute to the formulation of effective environmental policies. Such analyses are lacking for Lake Taihu.

The main objective of our study is, thus, to better understand the sources of river export of different nutrients forms (external nutrient loads) to Lake Taihu in relation to the critical nutrient loads. To this end, we implement the MARINA-Lake model (Model to Assess River Inputs of Nutrients to seAs) to quantify river export of $\mathrm{N}$ and $\mathrm{P}$ in different forms (dissolved inorganic and dissolved organic) from sub-basins draining into Lake Taihu. We focus on dissolved $\mathrm{N}$ and $\mathrm{P}$, since the bioavailability for harmful algae is considered higher for dissolved than for particulate forms (Garnier et al., 2010). We link the results of the MARINA-Lake model to the PCLake model to analyze to what extent the river export of $\mathrm{N}$ and $\mathrm{P}$ exceeds the critical loads. Finally, we discuss the opportunities for nutrient reduction at the sub-basin scale, based on the modeled sources of nutrients in the rivers. 


\subsection{Methods}

\subsubsection{Lake Taihu}

Lake Taihu is a large shallow sub-tropical lake located in Jiangsu province (Figure 3.1). The lake extends $2350 \mathrm{~km}^{2}$ and has an average depth of $2 \mathrm{~m}$. The water retention time of the lake is about half a year. In response to regular flooding, the lake has been dammed by a dike surrounding the lake. About 117 rivers and tributaries discharge into the lake (Xu et al., 2015a).

In this study, we defined a drainage area of Taihu basin covering $15,723 \mathrm{~km}^{2}$. We divide the drainage area into five sub-basins (I-V) according to the flow direction of $\mathrm{Wu}$ et al. (2011) at 5' resolution (Figure 3.1). Sub-basins I and III are upstream sub-basins draining into downstream sub-basins. Sub-basins II, IV, and V are downstream sub-basins draining into Lake Taihu. The upstream and downstream sub-basins cover $49 \%$ and $51 \%$ of the total basin area, respectively.

Taihu basin is located in an area with rapid urbanization and fast socio-economic development with a population of 10.5 million people in 2012 (Figure S1 in Appendix II). Around $70 \%$ of the population lives in urban areas. And more than $85 \%$ of the people live in Sub-basins I, II and IV. In urban areas, the people are relatively well connected (more than $70 \%$ ) to sewage systems, whereas the rural population is less well connected (less than 2\%). Increasing food demand has driven intensive agricultural production in the Taihu basin. As a result, agriculture has become an important contributor to $\mathrm{N}$ and $\mathrm{P}$ inputs to the basin (Figures S2, S3 in Appendix II). 


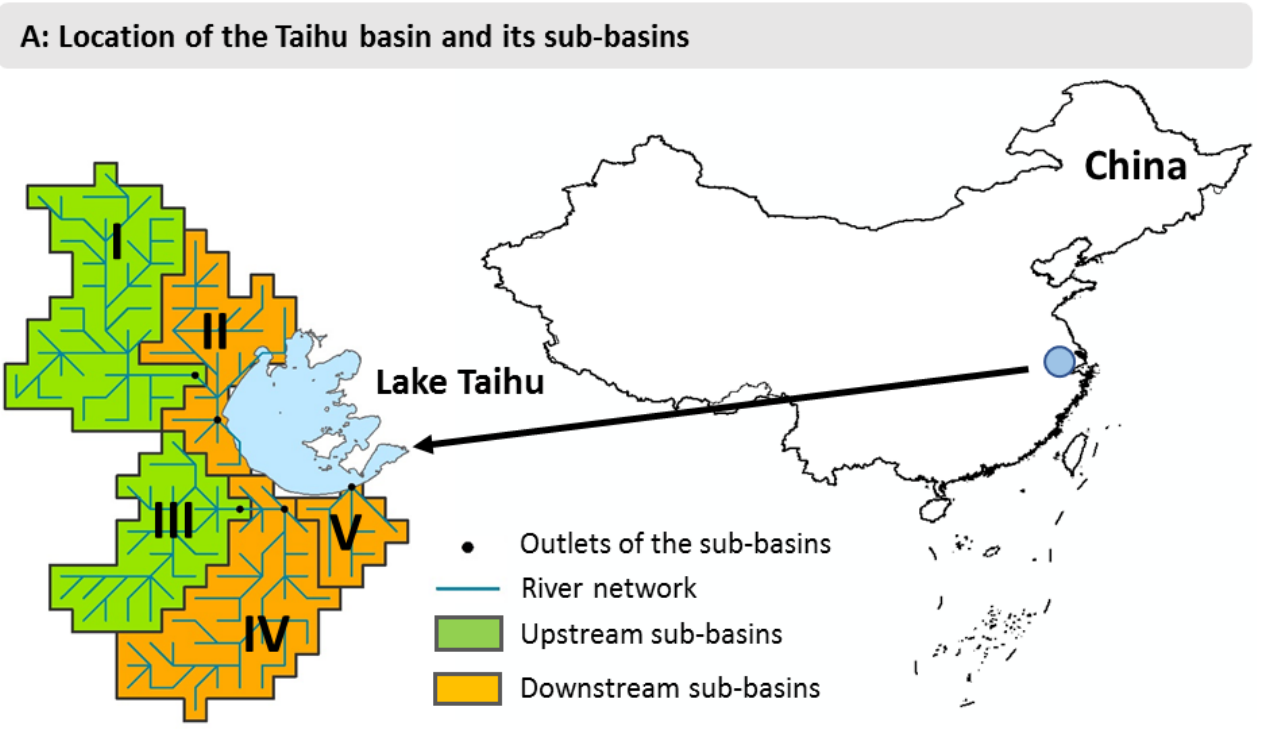

B: Overview of the sub-basin scale modelling for Taihu basin

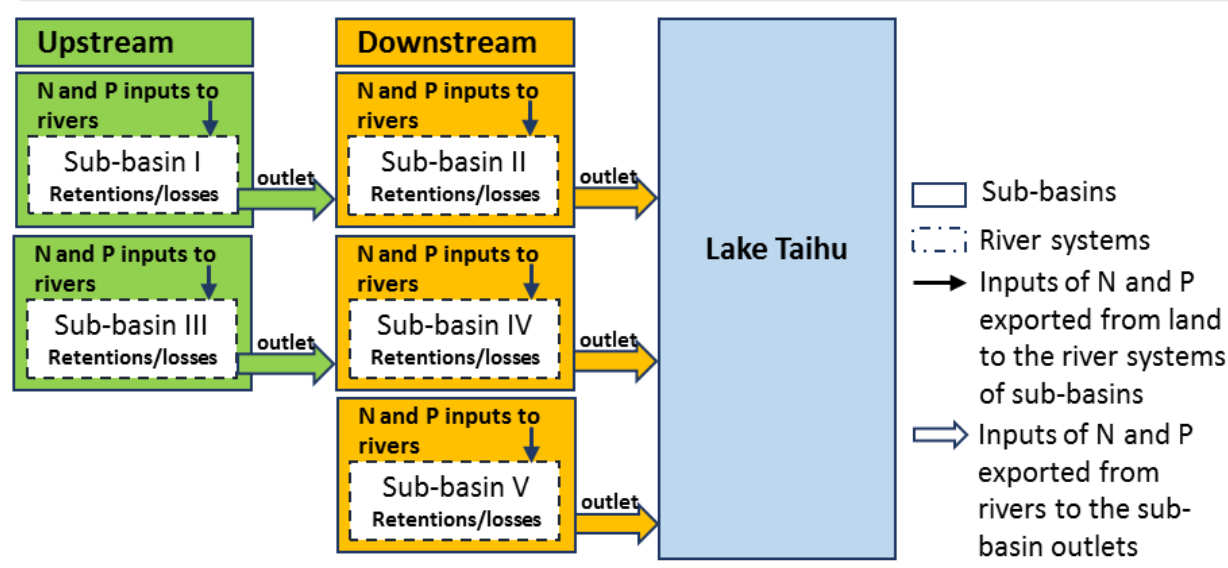

Figure 3.1 (A) Sub-basins draining into Lake Taihu, located in China; (B) The overview of the subbasin scale modeling for Taihu basin. The sub-basins are delineated based on the 5 ' resolution river network of Wu et al. (2011). The border for China is from RESDC (2013). 


\subsubsection{Model implementation}

Our study was performed in three steps. First, we quantified river export of nutrients to Lake Taihu using the MARINA-Lake model. MARINA-Lake is a version of the MARINA 1.0 model specifically focusing on river export of nutrients to lakes. Second, we used results from the PCLake model to identify critical nutrient loads for Lake Taihu. In the last step, we identified to what extent the river export of nutrients exceeds the critical load (nutrient reduction gap) by comparing the output from the MARINA-Lake model with the critical nutrient loads from the PCLake model, and discussed possibilities to reduce the nutrient exceedance gap.

\subsubsection{The MARINA-Lake model for river export of nutrients}

We applied the MARINA-Lake (Model to Assess River Inputs of Nutrients to seAs) model to quantify river export of dissolved inorganic (DIN, DIP) and organic (DON, DOP) N and P to Lake Taihu for 2012. The original MARINA 1.0 model was developed for Chinese rivers at the sub-basin scale (Strokal et al., 2016a). This model quantifies annual river export of $\mathrm{N}$ and $\mathrm{P}$ in different forms: dissolved inorganic and dissolved organic. River export of dissolved inorganic and organic $\mathrm{N}$ and $\mathrm{P}$ is quantified from diffuse (e.g., use of synthetic fertilizers, manure on land) and point (e.g., direct discharge of animal manure, sewage systems) sources. This model has been recently used to model several lakes in China, resulting in the MARINA-Lake model. The MARINA-Lake model has been used so far to analyze the sources of dissolved inorganic and organic $\mathrm{N}$ and P pollution for the past and in the future (up to 2050) in a spatially explicit way for three lakes: Dianchi (Li et al., 2019b), Bayandian and Guanting (Yang et al., 2019).

The MARINA-Lake model quantifies annual river export of $\mathrm{N}$ and $\mathrm{P}$ by sub-basins and by source to Lake Taihu based on the overall equation (Eq.1) as follows:

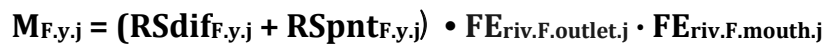

where

MF.y.j is river export of forms F (DIN, DON, DIP, DOP) to the river mouth by source y from sub-basin $\mathrm{j}$ (kg/year). The model distinguishes $\mathrm{N}$ and $\mathrm{P}$ inputs to rivers between diffuse sources and point sources. 
RSdif $_{\text {.y.j.j }}$ refers to inputs of nutrient form F to river systems (surface waters) from diffuse source y in sub-basin j. Diffuse sources included in this model are synthetic fertilizers, animal manure, human waste, atmospheric $\mathrm{N}$ deposition (for DIN) and biological $\mathrm{N}_{2}$ fixation (for DIN) over agricultural land, and atmospheric $\mathrm{N}$ deposition (for DIN) and biological $\mathrm{N}_{2}$ fixation (for DIN) over natural land.

RSpntF.y.j refers to inputs of nutrient form F to river systems (surface waters) from point source y in sub-basin j. Point sources include human waste from the sewage systems, human waste from the urban and rural population that is not connected to sewage systems and direct discharge of animal manure to rivers.

FEriv.F.outlet,j is the fraction of (RSdiff.y.j + RSpntF.y.j) exported to the outlet of sub-basin $j$ accounting for the retention of nutrient form $\mathrm{F}$ within the sub-basins.

FEriv.F.mouth.j is the fraction of (RSdiff.y.j + RSpntF.y.j) FEriv.Foutlet.j exported to the river mouth accounting for the retention of nutrient form $\mathrm{F}$ between sub-basin outlets and the river mouth. Both the FEriv.F.outlet.j and FEriv.F.mouth.j are calculated based on nutrient retention by denitrification, water consumption, and dams and lakes in the river systems. The detailed

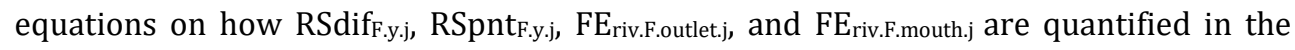
MARINA-Lake model is available in the Box S1 in Appendix II.

We took the MARINA-Lake model (Yang et al., 2019) and updated the model inputs for Lake Taihu (see Figure S5 in Appendix II). The model inputs for diffuse sources of $\mathrm{N}$ and $\mathrm{P}$ inputs on land (e.g., synthetic fertilizers, animal manure, biological $\mathrm{N}_{2}$ fixation) were derived from the NUFER model (NUtrient flows in Food Chains, Environment and Resources use) (Wang et al., 2018b). The model inputs for point sources of $\mathrm{N}$ and $\mathrm{P}$ to river systems (e.g., $\mathrm{N}$ and $\mathrm{P}$ in human excretion, population with sewage connection, $\mathrm{N}$ and $\mathrm{P}$ removal by sewage treatment) were updated with gross domestic product (GDP) and population densities from Chinese statistic yearbook (RESDC, 2013), information on sewage systems from the dataset for sewage treatment plants from Ministry of Ecology and Environment of the People's Republic of China (MEP, 2013b) and the study on Lake Taihu by Liu et al. (2013a). The model inputs for hydrology (e.g., river discharge, water consumption in the river systems) were derived from the Community WATer Model (CWATM) (Burek et al., 2017). CWATM is a distributed rainfall-runoff-routing model that 
includes all necessary hydrological processes for addressing water supply and demand modeling.

\subsubsection{The PCLake model for critical nutrient loads}

We compared the results from MARINA-Lake for nutrient export by rivers (sub-basins) to Lake Taihu with the critical nutrient loads as calculated by Janssen et al. (2017) (Figure 3.3). These critical nutrient loads were calculated with the water quality model PCLake and based on four water quality thresholds for phytoplankton chlorophyll-a starting at strict $(<20 \mu \mathrm{g} / \mathrm{L})$ to more tolerant $(<50 \mu \mathrm{g} / \mathrm{L}$ ) levels (Table S5 in Appendix II). The level of the thresholds depends on the purpose of water use: drinking water has higher water quality requirements than, for example, water for irrigation (Edition, 2011; Fewtrell and Bartram, 2001). Water quality will start to deteriorate when nutrient levels exceed the lower critical nutrient loads. Most of the in-lake chlorophyll-a will be higher than the threshold when nutrient levels exceed the upper critical nutrient load. River export of nutrients as calculated by MARINA-Lake are compared to the dissolved fraction of the critical nutrient loads: $50 \%$ of total $\mathrm{P}$, and $90 \%$ of total $\mathrm{N}$ is in dissolved form. Combining the calculated nutrient losses by source with critical nutrient loads will reveal possible options for nutrient management.

\subsection{Results}

We first evaluate the model performance in Section 3.3.1. Second, we present the results for river export of DIN, DON, DIP and DOP and their sources for the year 2012 in Section 3.3.2. Next, we compare how our modeled river export of TDN and TDP exceeds the critical nutrient loads and show the opportunities to meet the critical loads in Section 3.3.3.

\subsubsection{Model performance}

We evaluated the results from the MARINA-Lake model for Lake Taihu in three ways.

First, we compared our modeled fluxes of $\mathrm{N}$ and $\mathrm{P}$ with the measured fluxes of $\mathrm{N}$ and $\mathrm{P}$ in the lake (Table 3.1, and Table S4 in Appendix II). Measured fluxes of N and P to Lake Taihu were available from Xu et al. (2015a) and Li et al. (2011b). The comparison shows we model somewhat higher $\mathrm{N}$ inputs into the lake compared to the measurements. There are a number of explanations for this. One is the difference in the studied period and location: our model is for 2012 and the measurements are for the period 2000 to 2012. We model 
the river export of nutrients (external loads into the lake) whereas the measurements might be from water samples at different locations in the lake. Differences in nutrient forms are another reason: we model river export of dissolved inorganic and organic nutrients whereas the measurements are for total $\mathrm{N}$ and $\mathrm{P}$ thus including particulate forms. Furthermore, our model quantifies annual river export of $\mathrm{N}$ and P. However, the measurements are often derived from water samples collected at a specific time during certain periods (e.g., days, seasons) and then averaged to annual values.

Second, we compared our modeled river export of $\mathrm{N}$ and $\mathrm{P}$ with some other modeling studies (Table 3.1, and Table S4 in Appendix II). Only few studies subdivide the draining area of Taihu basin into sub-basins (e.g., Yu et al. (2007)) and they delineated sub-basins using a different approach than the one we took. Therefore, we compared our results with other studies at the basin scale rather than at the sub-basin scale. Several studies (e.g., Liu et al., 2013a) quantified nutrient loads into Lake Taihu using, for example, the SWAT model (e.g., Lai et al., 2006). The comparison shows that our modeled results are within the range of the results from other studies (Table 3.1). For example, we model 61 kton of $\mathrm{N}$ and 2.0 kton of $\mathrm{P}$ exported by rivers in 2012. The other studies estimate 33-66 kton of $\mathrm{N}$ and 1.6-5.2 kton of $\mathrm{P}$ for different years (1995-2012, see Table 3.1).

Third, we compared the source attribution from the MARINA-Lake model with some existing studies (Table S4 in Appendix II). Results of this comparison are promising and show that our results are generally in line with other studies. For example, the other studies emphasize the importance of agriculture in nutrient pollution of the lake. The results of the MARINA-Lake model are in line with this conclusion. More information on comparing our results with other studies and on model uncertainties can be found in Section 3.4.1.

Based on this model evaluation, we consider the performance of the MARINA-Lake model good enough for analyses of river export of nutrients, and their sources, to Lake Taihu. As mentioned above, the MARINA-Lake model has been successfully implemented to a few other lakes in China: Dianchi (Li et al., 2019b), Bayandian and Guanting (Yang et al., 2019). This gives trust in implementing the MARINA-Lake model for Lake Taihu. 
Table 3.1 Comparison of our modeled annual river export of total $\mathrm{N}$ and $\mathrm{P}$ to Lake Taihu with other studies. Our study is in the grey shaded row.

\begin{tabular}{|c|c|c|c|c|c|c|}
\hline \multirow[t]{2}{*}{ Studies } & \multicolumn{2}{|l|}{$\mathrm{TN}$} & \multicolumn{2}{|l|}{$\mathrm{TP}$} & \multirow{2}{*}{$\begin{array}{l}\text { Temporal } \\
\text { resolution }\end{array}$} & \multirow[t]{2}{*}{ Type of study } \\
\hline & $\mathrm{g} / \mathrm{m}^{2} /$ year & kton/year & $\mathrm{g} / \mathrm{m}^{2} /$ year & kton/year & & \\
\hline \multirow{2}{*}{ Xu et al. (2015a) } & 20 & 46 & 0.9 & 2.1 & $2007-2012$ & Measurements \\
\hline & $(17-23)$ & $(41-54)$ & $(0.8-1.1)$ & $(1.8-2.6)$ & & \\
\hline Li et al. (2011b) & $9.3-12 *$ & $21-27$ & $0.4-0.5$ & $0.8-1.2$ & $2000-2005$ & Measurements \\
\hline Liu et al. (2013a) & $14.7^{*}$ & 33 & 2.3 & 5.23 & 2008 & Modeled results \\
\hline Huang et al. (2018) & - & - & 0.8 & 1.9 & 2014-2016 & $\begin{array}{l}\text { Modeled results, } \\
\text { polders }\end{array}$ \\
\hline $\begin{array}{l}\text { Reidsma et al. } \\
\text { (2012) }\end{array}$ & $29.3^{*}$ & 66 & 0.7 & 1.6 & 2008 & Modeled results \\
\hline Lai et al. (2006) & $\begin{array}{l}17.8^{*} \\
(16.4-18.7)^{*}\end{array}$ & $\begin{array}{l}40 \\
(37-42)\end{array}$ & $\begin{array}{l}0.9 \\
(0.8-0.9)\end{array}$ & $\begin{array}{l}2.0 \\
(1.8-2.0)\end{array}$ & $\begin{array}{l}\text { 1995, 1998, } \\
2002\end{array}$ & Modeled results \\
\hline This study** & 27 & 61 & 0.9 & 2.0 & 2012 & Modeled results \\
\hline
\end{tabular}

${ }^{*}$ Estimated from kton/year using the surface area of the lake. ${ }^{* *}$ In this study we model total dissolved $\mathrm{N}$ and $\mathrm{P}$, which is the sum of dissolved inorganic and organic $\mathrm{N}$ and $\mathrm{P}$.

\subsubsection{River export of nutrients and their sources}

River export of nutrients differed among sub-basins in 2012. Rivers exported 61 kton of TDN and 2 kton of TDP to Lake Taihu in 2012 (Figure 3.2). Most of these nutrients were in dissolved inorganic forms. More than half of the nutrients resulted from activities in Sub-basins I and IV that cover around $60 \%$ of the Taihu basin drainage area. Sub-basin II contributed by $15-22 \%$ to the nutrients transported to Lake Taihu depending on the nutrient form. The share of Sub-basin III in the total nutrient loads to the lake was estimated at $8-16 \%$ for different nutrient forms. Sub-basin V contributed $<10 \%$ to the nutrients in 2012 (Figure 3.2). Rivers of Sub-basin III exported the smallest amount of these nutrients per $\mathrm{km}^{2}$ whereas rivers of Sub-basin IV exported the largest amounts of the nutrients per $\mathrm{km}^{2}$ in 2012 (Figure S6 in Appendix II).

Diffuse sources were responsible for about $90 \%$ of the total TDN river export to Lake Taihu in 2012 (Figures 3.2 and 3.3). Around 40\% of this TDN river export from diffuse sources was from synthetic fertilizers. However, the shares of diffuse sources varied greatly among sub-basins and $\mathrm{N}$ forms (Figure 3.2). For example, the share of diffuse sources ranged from 83 to $98 \%$ for DIN and from 43 to $89 \%$ for DON among the subbasins (Figure 3.2). Use of synthetic fertilizers on land was an important diffuse source of DIN in rivers of all sub-basins. Atmospheric $\mathrm{N}$ deposition also contributed to DIN in rivers of the sub-basins (16-30\% in the total DIN export). This was different for DON. Leaching of organic matter was an important source of DON in rivers (with a share of $36-74 \%$ for 
different sub-basins and $\mathrm{N}$ forms). In addition, sewage systems (point source) were responsible for half of DON in rivers of Sub-basin II and for 7-38\% of DON in rivers of the other sub-basins.

Point sources were responsible for $48 \%$ of the total TDP river export to Lake Taihu in 2012 (Figures 3.2 and 3.3). Most of this TDP from point sources were from sewage systems. This varied among sub-basins and P forms (Figure 3.2). The share of sewage systems in DIP river export ranged from 11 to 60\% among sub-basins. In rivers of Subbasins II and V, more than $40 \%$ of DIP resulted from sewage systems, indicating rapid urbanization in these sub-basins. In addition, diffuse sources namely use of synthetic fertilizers (for Sub-basins I, III and IV) and animal manure on land (for Sub-basins I, III, IV, and V) were important sources of DIP in rivers. For river export of DOP, direct manure discharges were more important point sources of DOP in rivers than sewage systems. The share of manure discharges in DOP river export ranged from 22\% (Sub-basin III) to $42 \%$ (Sub-basin V) among sub-basins (Figure 3.2). Leaching of organic matter, use of animal manure and synthetic fertilizers also contributed to DOP in rivers. 

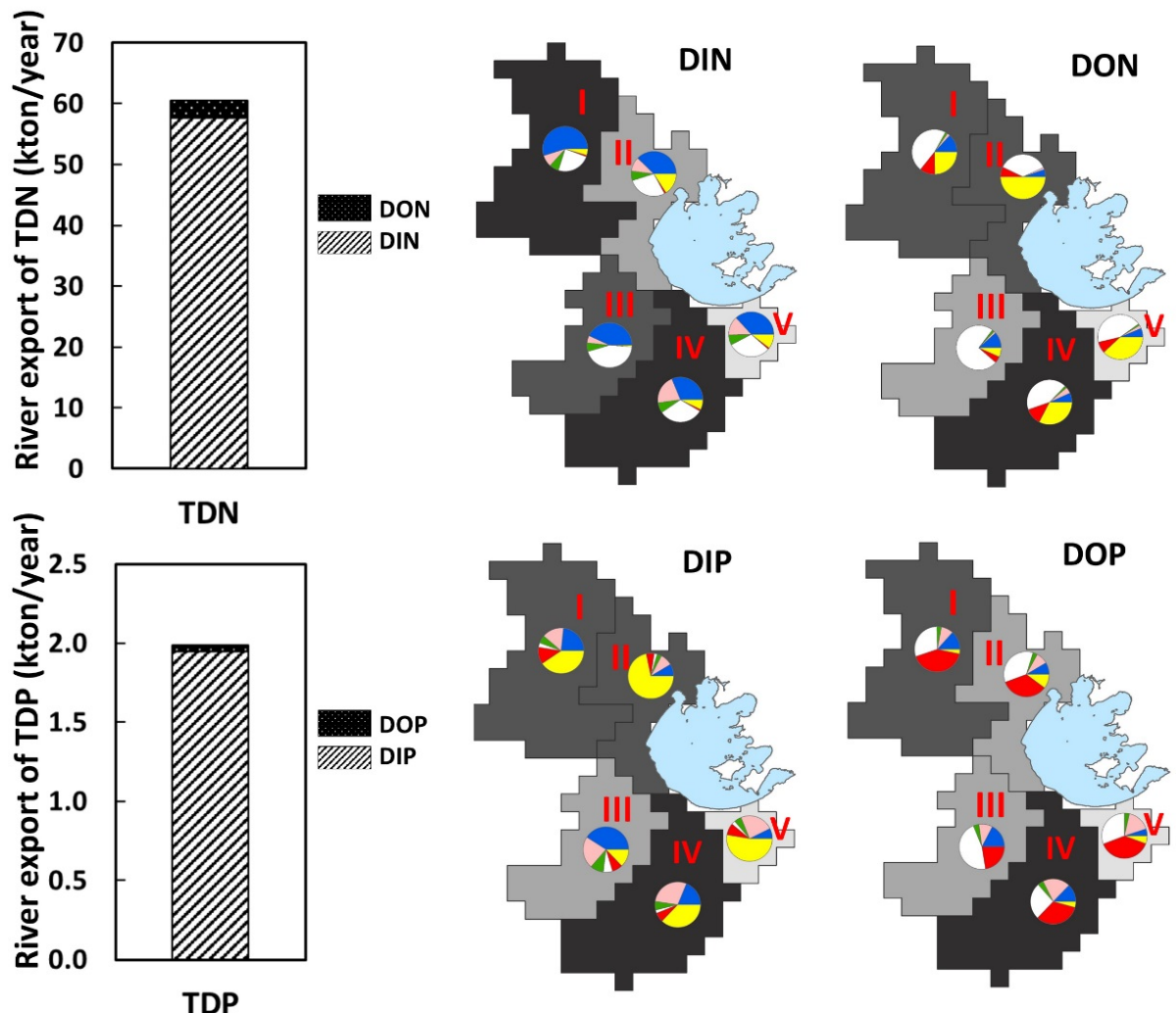

\section{River export of nutrients (kton/year)}

\begin{tabular}{|c|c|c|c|c|c|c|}
\hline DIN & & DON & DIP & & DOP & \\
\hline & $9.1-20.7$ & $0.7-1.0$ & & $0.42-0.83$ & & $0.010-0.016$ \\
\hline & $8.8-9.1$ & $0.4-0.7$ & & $0.16-0.42$ & & $0.006-0.010$ \\
\hline & $3.8-8.8$ & $0.2-0.4$ & & $0.15-0.16$ & & $0.003-0.006$ \\
\hline & $0-3.8$ & $0-0.2$ & & $0-0.15$ & & $0-0.003$ \\
\hline
\end{tabular}

\section{Share of the sources in river export of nutrients (fraction)}

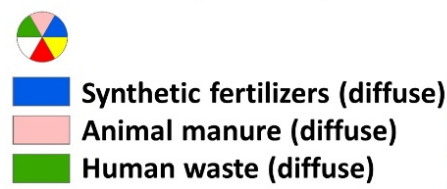

Other sources (diffuse) Animal manure (point) Sewage systems (point)

Figure 3.2 River export of nitrogen and phosphorus by source from sub-basins to Lake Taihu in 2012. TDN and TDP are total dissolved nitrogen and phosphorus, respectively. DIN and DIP are dissolved inorganic nitrogen and phosphorus, respectively. DON and DOP are dissolved organic nitrogen and phosphorus, respectively. "Others" include atmospheric $\mathrm{N}$ deposition over agricultural and non-agricultural areas (for DIN), biological $\mathrm{N}_{2}$ fixation by natural vegetation and crops (e.g., legumes) (for DIN), leaching of organic matter (for DON and DOP) and weathering of P-contained minerals (for DIP) from agricultural and non-agricultural areas. The results are from the MARINA-Lake model (see Section 3.2.2). 


\subsubsection{Opportunities to meet critical nutrient loads}

In this section, we identify opportunities to meet critical nutrient loads for Lake Taihu. To this end, we compare the actual river export of nutrients with the critical loads to Lake Taihu to identify the required reductions.

Critical nutrient loads to Lake Taihu depend on the desired level of Chlorophyll-a in the lake (Section 3.2.2.2, Table S5 in Appendix II). We present results for the two extreme levels of Chlorophyll-a: $20 \mu \mathrm{g} / \mathrm{L}$ (most strict) and $50 \mu \mathrm{g} / \mathrm{L}$ (least strict). For $20 \mu \mathrm{g} / \mathrm{L}$ of Chlorophyll-a, the most strict critical load is $5 \mathrm{kton} /$ year for $\mathrm{N}$ and $0.15 \mathrm{kton} / \mathrm{year}$ for $\mathrm{P}$. For $50 \mu \mathrm{g} / \mathrm{L}$ of Chlorophyll-a, the least strict critical load is $33 \mathrm{kton} / \mathrm{year}$ for $\mathrm{N}$ and 0.9 kton/year for P (Table S5 in Appendix II). In our analysis, we compare the modeled actual river export of $\mathrm{N}$ and $\mathrm{P}$ in kton/year with these critical loads (Figure 3.3). Water quality that meets the critical nutrient loads of $20 \mu \mathrm{g} \mathrm{Chl}-\mathrm{a} / \mathrm{L}$ can be considered as very good and appropriate for, for example, drinking purposes (Janssen et al., 2017). Water quality that meets the critical nutrient loads of $50 \mu \mathrm{g} \mathrm{Chl-a/L} \mathrm{can} \mathrm{be} \mathrm{considered} \mathrm{as} \mathrm{just} \mathrm{acceptable,} \mathrm{and}$ is more appropriate for purposes such as irrigation (Edition, 2011; Fewtrell and Bartram, 2001).

Results show that the actual river export of nutrients exceeded largely the critical loads in 2012 (Figure 3.3). To meet critical loads of TDN, river export needs to be reduced by 46-92\%, depending on the level of Chlorophyll-a. This implies that river export of TDN needs to be reduced by around 28 kton to meet the least strict critical N load (50 $\mu \mathrm{g} \mathrm{Chl-}$ $\mathrm{a} / \mathrm{L}$ ). To meet the most strict critical $\mathrm{N}$ load (aiming for $20 \mu \mathrm{g} \mathrm{Chl-a/L}$ ), the required reduction is around 56 kton for TDN. Most TDN in river export was DIN (see Figure 3.2 and Section 3.3.2). Thus, the required reductions apply to DIN. Most DIN in rivers draining in Taihu was from diffuse sources with a large share of synthetic fertilizers (see Figures 3.2 and 3.3, Section 3.3.2). Thus, improving $\mathrm{N}$ use efficiencies in agriculture (e.g., use of fertilizers according to crop needs) may effectively reduce DIN in rivers to meet the least strict critical N load. However, to meet the most strict critical N load (aiming for $20 \mu \mathrm{g} \mathrm{Chl-}$ a/L), additional reductions in DIN in rivers from other sources are required.

To meet critical loads of TDP, river export needs to be reduced by 55-92\%, depending on the level of Chlorophyll-a (Figure 3.3). We estimate a required reduction of 1.1 kton for TDP river export from all sub-basins to meet the least strict critical P load (aiming for 50 $\mu \mathrm{g}$ Chl-a/L). A reduction of $1.8 \mathrm{kton}$ in TDP is needed to meet the most strict critical P load 
$(20 \mu \mathrm{g} \mathrm{Chl-a/L).} \mathrm{Most} \mathrm{TDP} \mathrm{river} \mathrm{export} \mathrm{is} \mathrm{DIP} \mathrm{(see} \mathrm{Section} \mathrm{3.3.2,} \mathrm{Figure} \mathrm{3.2).} \mathrm{Thus,}$ reducing DIP in rivers can largely contribute to meet the critical P loads in Lake Taihu. Our results show that $11-60 \%$ of the DIP in river export from sub-basins was from point sources (sewage systems). Thus improving removal efficiencies for nutrients during wastewater treatment in sewage systems is an effective way to meet the least strict critical P load. However, meeting the most strict critical P load will be difficult without considering other sources (e.g., use of synthetic fertilizers and animal manure on land, Figures 3.2 and 3.3). Combining better nutrient management in agriculture with improved sewage treatments is needed to meet the critical loads. 

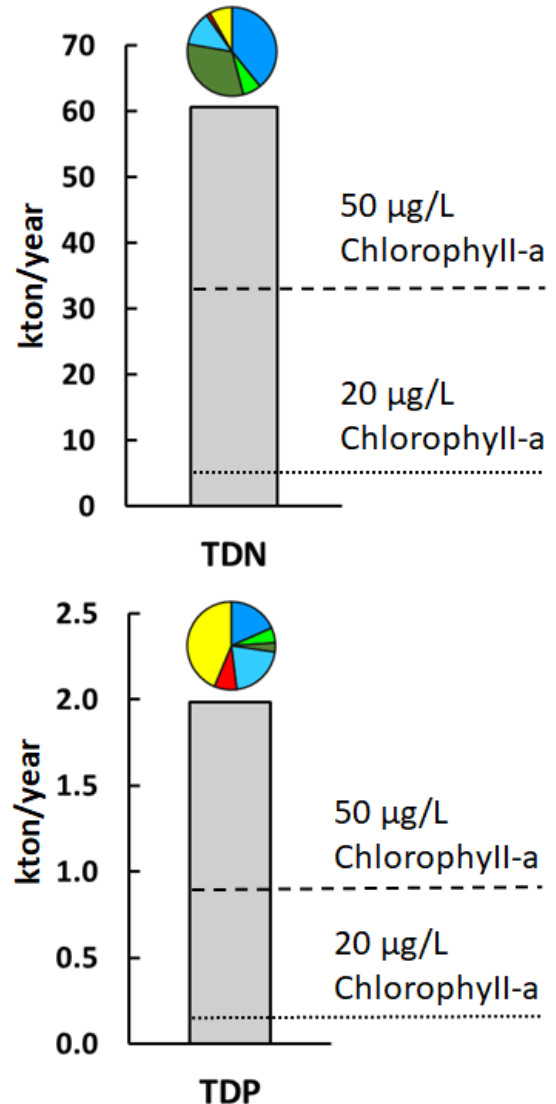

Legends:

Actual river export of TDN and TDP in 2012 (kton/year)

Sources in actual river export of TDN and TDP (0-1) Synthetic fertilizers (diffuse)

Animal manure (diffuse)

Human waste (diffuse)

Other sources (diffuse)

Animal manure (point)

Sewage systems (point)

- - - Critical TDN and TDP loads to Lake Taihu, depending on the chlorophyll-a level

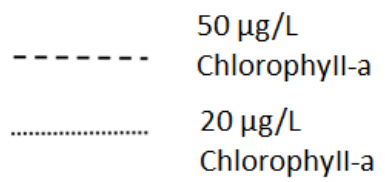

Figure 3.3 Actual river export of total dissolved nitrogen (TDN) and phosphorus (TDP) in 2012 (kton/year, shown by the bars), the share of the sources (0-1, shown by the pies) and the critical TDN and TDP loads (kton/year, shown by the dash lines) according to the two levels of Chlorophyll-a (20 and $50 \mu \mathrm{g} / \mathrm{L}$ ). Actual river export of TDN and TDP is from the MARINA-Lake model (see Sections 3.2.2, 3.3.2). TDN and TDP are the sum of dissolved inorganic (DIN, DIP) and organic (DON, DOP) nitrogen and phosphorus. "Others" include atmospheric N deposition over agricultural and non-agricultural areas (for DIN), biological N2 fixation by natural vegetation and crops (e.g., legumes) (for DIN), leaching of organic matter (for DON and DOP) and weathering of P-contained minerals (for DIP) from agricultural and non-agricultural areas. The critical TDN and TDP loads according to the levels of Chlorophyll-a are from the PCLake model (see Section 3.2.2.2) (Janssen et al., 2017). The levels of chlorophyll-a of 20 and $50 \mu \mathrm{g} / \mathrm{L}$ were used to determine the levels of the critical loads of TDN and TDP in Lake Taihu (see Table S5 in Appendix II for details). 


\subsection{Discussion}

\subsubsection{Sources of nutrient export to Lake Taihu}

We linked the MARINA-Lake model with the PCLake model for Lake Taihu to better understand the sources of river export of nutrients to Lake Taihu with respect to the critical nutrient loads of the lake. In general, our model results compare well with the existing studies (see also Section 3.3.1). This also holds for the source attribution. For example, we estimate that around $15 \%$ of $\mathrm{N}$ and $49 \%$ of $P$ river export from all sub-basins resulted from households (sewage systems and human waste as well as fertilizers, see Table S4 in Appendix II). This is in the range of other studies indicating that the share of households is $17-43 \%$ for $\mathrm{N}$ and $28-50 \%$ for $\mathrm{P}$ (Lai et al., 2006; Wang et al., 2006). We estimate that 1-8\% of $\mathrm{N}$ and $\mathrm{P}$ in Lake Taihu is from direct discharge of animal manure to rivers. Exiting studies estimate 5-20\% (Liu et al., 2013a; Wang et al., 2006). Our estimates for the share of livestock activities (use of manure on land and direct discharges) are in line with the estimates of Liu et al. (2013a) (see Table S4 in Appendix II).

There are also some differences between our study and other studies (Table S4 in Appendix II). For example, our study estimates a larger share of diffuse sources for $\mathrm{N}$ than existing studies (e.g., Cao et al. (2013); Lai et al. (2006); Wang et al. (2006)). The difference can be explained by the fact that we account for more sources of $\mathrm{N}$ in rivers (e.g., biological $\mathrm{N}_{2}$ fixation) from agricultural and non-agricultural areas. Another reason is that there is a difference in the study areas and the level of temporal detail between our study and existing studies (Table 3.1). This may explain the differences between our estimates and other studies for sources of nutrients in rivers of the Taihu basin. Furthermore, we calculated slightly higher contributions from sub-basins III, IV and V in the south than from sub-basins I and II in the north, which was also found by Yu et al. (2007). Our results show sub-basins III, IV and V contributed in total $56-61 \%$ to the nutrients transported to Lake Taihu depending on the nutrient form. This spatial pattern can be conceived counterintuitive as most cyanobacterial growth was observed in the north (Duan et al., 2009; Li et al., 2018). However, due to hydrological patterns that limit the exchange of water between the bays and the rest of the lake, the nutrient load in the northern bays of the lake is spread over a relatively small area. The nutrients from the northern sub-basins are mixed over a large part of Lake Taihu (Li et al., 2011c). When nutrient loads are spread over a relatively small area, the result is locally higher nutrient concentrations that boost 
algal growth. Besides the reason of the nutrient loads, the effect of wind is another important reason to explain higher algal biomass in the north. The general wind direction at Lake Taihu is towards the northwest. In the center of Lake Taihu, the wind is generally too strong to sustain high algal growth. Besides, algae that grow in the center of the lake are blown to the northern parts of the lakes.

With respect to the uncertainties in critical nutrient loads, Janssen et al. (2017) made a comparison with Xu et al. (2015a). Xu et al. (2015a) estimated a required reduction of 20$71 \%$ for nutrients in the lake to meet the critical nutrient threshold (condition of $<20 \mu \mathrm{g}$ $\mathrm{L}^{-1}$ chlorophyll a). This is lower than our study (46-92\%, see Figure 3.3). The reason for this can be that Xu et al. (2015a) calculated the reduction for TN and TP, while in this study we focus on TDN and TDP. In addition, Xu et al. (2015a) used experiments to determine the critical nutrient thresholds whereas the critical nutrient loads in this study are obtained using the model PCLake (Section 3.2.2).

\subsubsection{Combing MARINA-Lake and PCLake: strengths and limitations}

We combined the sub-basin scale MARINA model and the lake ecosystem model PCLake. This approach allows us to identify opportunities to reduce the gap between the actual and critical nutrient loads. To our knowledge, such a comparison was not done before for Lake Taihu. This comparison is very useful for Lake Taihu since many existing studies have shown that eutrophication problems are increasing in this lake in the last decades (Duan et al., 2009; Janssen et al., 2014; Qin et al., 2010).

There are also limitations in combing these two models. For example, to compare the critical nutrient loads with the results from MARINA, we converted critical loads for total $\mathrm{N}$ and $\mathrm{P}$ to dissolved $\mathrm{N}$ and $\mathrm{P}$ (Section 3.2.2). This means that the gap between the actual loading and the critical nutrient loads in our study needs to be interpreted in the light of dissolved forms. Since dissolved forms of $\mathrm{N}$ and $\mathrm{P}$ generally are considered to have higher bioavailability for harmful algae than their particulate forms (Garnier et al., 2010), we consider this as an acceptable approach to combine the results of two models.

Our model has uncertainties associated with model inputs, parameters, and nutrient sources. For example, we derived many model inputs for agricultural activities (e.g., use of synthetic fertilizers, manure excretion) from the NUFER model (Wang et al., 2018b). This model provides inputs for over three thousands counties in China. We aggregated county-scale inputs to sub-basin-scale inputs using an area-weighted method in ArcGIS 
(see Table S3 in Appendix II for more explanation). Model inputs for water discharge at the sub-basin outlets were derived from the CWATM model at 5 min resolution. This model was not calibrated for rivers draining to Lake Taihu. We realize that an uncalibrated hydrological model may introduce uncertainties in our estimates. For example, a higher discharge will lead to more nutrients exported to Lake Taihu which increases algal blooming and requires stronger nutrient reductions (Paerl and Huisman, 2009). However, to calibrate the model an extensive number of measurements at the outlets of the Taihu sub-basins are needed which are not at our disposal. Some of the MARINA-Lake model parameters were based on literature and in this study do not vary among sub-basins (e.g., the fraction of manure discharges to rivers, Section 3.2.2). Regarding the nutrient sources, the MARINA-Lake model ignores sources such as $\mathrm{P}$ deposition from atmosphere and aquaculture. To our knowledge, these sources do not contribute largely to the total nutrient pollution in Lake Taihu (e.g., Cai et al. (2013); Liu et al. (2013a); Wang et al. (2006)) compared to agricultural activities and sewage systems. The PCLake model is well known in the context of critical nutrient loads (Janse et al., 2008; Janssen et al., 2014; Kong et al., 2017; Li et al., 2019b). PCLake quantifies critical nutrient loads based on the average temporal and spatial conditions in the lake. In reality, conditions of lakes fluctuate among years, such as wetter or dryer years and warmer or colder years. These fluctuations cause uncertainties in the critical nutrient loads when applied for specific years. Moreover, Janssen et al. (2017) showed an application of the PCLake model in which spatial differences in critical nutrient loads exist: some parts of the lake have a lower critical nutrient load and are thus more sensitive to a shift than other parts of the lake. Our results should be seen, therefore, as an indication of the temporally and spatially averaged critical nutrient load of Lake Taihu.

We believe that the model uncertainties do not change the main messages of our study. We validated the MARINA-Lake model with measurements for water quality that are available to us. Validation results are promising (Section 3.3.1). The critical nutrient loads found with the PCLake model has been validated by Janssen et al. (2017). In addition, we compared our results with results of other existing studies for Lake Taihu (see Table 3.1, and Table S4 in Appendix II). This gives trust in using the MARINA-Lake model to analyze the sources of $\mathrm{N}$ and $\mathrm{P}$ in rivers to Lake Taihu. Furthermore, the MARINA-Lake model has been successfully applied to other lakes in China: Lake Dianchi (Li et al., 2019b) and 
Guanting Reservoir and Lake Baiyangdian (Yang et al., 2019). In the case of Lake Dianchi the combination of MARINA-Lake model with the PCLake model was also applied.

\subsubsection{Implications for environmental policies}

Our study can help to search for effective environmental policies for lake restoration. We show the gap between the actual river export of $\mathrm{N}$ and $\mathrm{P}$ and the critical nutrient loads (Section 3.3.3). Reducing this gap will reduce eutrophication problems and facilitate lake restoration. This gap depends on the requirements of water quality for different purposes of water use (e.g. drinking water or irrigation). In our study, we use the two extreme critical nutrient loads depending on the desired level of Chlorophyll-a: least strict (e.g., water for irrigation) and most strict (e.g., water for drinking) critical nutrient loads.

The need for effective environmental policies to restore Lake Taihu is recognized in existing studies (e.g., Liu et al. (2013a); Ma et al. (2014a); Reidsma et al. (2012)). For example, policies for sustainable agricultural developments are needed to avoid nutrient pollution of Lake Taihu (e.g., Ma et al. (2014a); Reidsma et al. (2012)). Our study provides useful information on from which human activities (sources) and areas (sub-basins) nutrient export by rivers should be reduced to meet the critical nutrient loads in Lake Taihu. We argue that reducing $\mathrm{N}$ inputs in rivers from synthetic fertilizers (diffuse source) and $\mathrm{P}$ inputs in rivers by improving treatment of wastewater (point source) are essential to meet the critical nutrient load under which water can be used for irrigation purposes. However, more efforts might be needed to meet the critical nutrient loads under which water can be used for drinking. This can be done by 1) fertilizing the crops according to their needs for nutrients, 2) recycling animal manure on land to avoid manure discharges to water, and 3) improving wastewater treatment in sewage systems of the Taihu basin (Strokal et al., 2017; Wang et al., 2018a; Zhang et al., 2012). Some of the current policies already aim to reduce nutrient loads in Lake Taihu. For example, implementing the 'Zero Fertilizer Policy' that aims for zero growth in the use of synthetic fertilizers after 2020 will reduce the fertilizer use in the Taihu basin (MOA, 2015c). The national government has also introduced management strategies and technologies for improving sewage treatment of nutrients in rural areas (Wang et al., 2010b). These measures will help to reduce nutrient inputs to the lake from rural sewage. Changes in climate are also important for future water quality in the lake because climate-related factors (e.g. temperature, wind speed) also have effects on changes in critical nutrient loads in Lake 
Taihu, and in nutrient retention in the Taihu basin (Zhang et al., 2018). Thus future policies should combine management options to reduce river export of $\mathrm{N}$ and $\mathrm{P}$ from both diffuse (e.g., use of synthetic fertilizers, manure) and point (e.g., sewage systems) sources, with consideration of future changes in climate. In any case, it is important to realize that a full restoration of Lake Taihu will take time, even with strong nutrient reductions, as it will take years before the large pool of nutrients stored in the sediments will be released (Wu et al., 2019).

\subsection{Conclusions}

Our results show that the sources of nutrient inputs to Lake Taihu differed largely among nutrient forms and sub-basins in 2012. Rivers exported 61 kton of TDN and 2 kton of TDP to Lake Taihu, mostly in dissolved inorganic forms. More than half of the nutrients in Lake Taihu were from Sub-basins II (north) and IV (south). Diffuse sources contributed $90 \%$ to the TDN in rivers. More than $40 \%$ of this TDN from diffuse sources was from synthetic fertilizers used on land. The share of diffuse sources in river export of $\mathrm{N}$ ranged from 43 to $98 \%$ among the sub-basins and $\mathrm{N}$ forms. Atmospheric $\mathrm{N}$ deposition was another important source of DIN and sewage systems of DON in rivers. Point sources contributed $52 \%$ to TDP in the rivers. Important point sources were sewage systems. The share of sewage systems in river export of DIP ranged from 11 to $60 \%$ among sub-basins. Direct manure discharges were important sources for river export of DOP.

To meet the critical nutrient loads, river export of TDN and TDP needs to be reduced by 46-92\%. This implies a reduction of 28-56 kton TDN and 1.1-1.8 kton TDP, depending on the level of Chlorophyll-a. In our analysis, we focus on the critical nutrient loads based on two extreme levels of Chlorophyll-a in the lake: $20 \mu \mathrm{g} / \mathrm{L}$ (most strict, e.g., for drinking purposes) and $50 \mu \mathrm{g} / \mathrm{L}$ (least strict, e.g., for irrigation purposes). The least strict nutrient loads may be reached by reducing river export of DIN from diffuse sources (synthetic fertilizers) and DIP from point sources (sewage systems). To meet the most strict nutrient loads, it requires combinations of reduction options for both diffuse and point sources. Examples are improved nutrient us e efficiency in agriculture by better meeting the needs of nutrients by crops, combined with best available technologies to remove nutrients in sewage systems.

Our study shows the main sources of nutrients in rivers draining into Lake Taihu. This can help to identify opportunities to meet critical nutrient loads. A good understanding of 
nutrient sources is essential for formulating effective management options to reduce nutrient pollution. Our study may thus contribute to restoring the ecosystems in the lake, and serve as an example for other lakes.

\section{Acknowledgments}

This study is funded by the National Key R\&D Program of China [project number 2016YFE0103100], the KNAW (Koninklijke Nederlandse Akademie van Wetenschappen) project SURE+ [project number PSA-SA-E-01], the NWO (Netherlands Organization for Scientific Research) project Multi Pollutant Modelling [project number 5160957330DEELPRJ_1, 2016-2018], and the WIMEK (Wageningen Institute for Environment and Climate Research) fellowship [project number 5160957306]. We thank Tim aus der Beek for the discussion on nutrient pollution in Lake Taihu, which inspired us to initiate this work. 



\title{
Chapter 4. Reactive Nitrogen Losses from China's Food System for the Shared Socio-economic Pathways (SSPs)
}

\begin{abstract}
Food production in China has been changing fast as a result of socio-economic development. This resulted in an increased use of nitrogen $(\mathrm{N})$ in food production, and also to increased reactive nitrogen $(\mathrm{Nr})$ losses to the environment, causing nitrogen pollution. Our study is the first to quantify future $\mathrm{Nr}$ losses from China's food system for the Shared Socio-economic Pathways (SSPs). We show that Nr losses differ largely among SSPs. We first qualitatively described the five SSP storylines for China with a focus on food production and consumption. Next, we interpreted these SSP scenarios quantitatively for 2030 and 2050, using the NUFER (NUtrient Flows in Food chains, Environment and Resources use) model to project the $\mathrm{Nr}$ losses from China's food system. The results indicate that $\mathrm{Nr}$ losses from future food system in China are relatively low for SSP1 and SSP2, and relatively high for SSP3 and SSP4. In SSP5 Nr losses from China's food system are projected to be slightly lower than the level of today.
\end{abstract}

Published as:

Wang M., Kroeze C., Strokal M., Ma L. Reactive nitrogen losses from China's food system for the shared socioeconomic pathways (SSPs). Science of The Total Environment 2017; 605-606: 884-893. 


\subsection{Introduction}

Food production and consumption in China have been changing fast because of the rapid economic growth and social transformations in the country. Important recent changes include: (i) a fast increase in food production and fertilizer application; (ii) an increasing share of horticultural cash crops and animal production in total food production; (iii) a shift towards larger and more intensive animal production farms, with less manure recycling due to decoupling of crop and animal production; (iv) an increasing demand for animal feed, and, as a result of that, an increase in the production of maize and import of soybean; (v) a change in food processing from micro-enterprises to large-scale food processing companies; (vi) a change in food distribution chains from traditional local markets to supermarket chains; (vii) a tendency to eat in restaurants in urban areas, and more variety in the human diet; (viii) an increase in food waste throughout the production chain from harvest to plate (Du et al., 2004; Garnett and Wilkes, 2014; Kearney, 2010; Schneider, 2011; UNEP, 2016a; Zhang et al., 2013). All these trends are driven by socioeconomic development, and have an impact on nutrient losses from the food system to the environment.

Agriculture is an important source of nitrogen $(\mathrm{N})$ in the environment. Nitrogen is essential for the growth of plants and animals, and is hence critical for the production of sufficient food for the large population in China (Ma et al., 2013b; Zhang et al., 2012). The increasing use of $\mathrm{N}$ for food production, has been associated with increased losses of reactive $\mathrm{N}(\mathrm{Nr}$ ) to the atmosphere and aquatic systems (Bai et al., 2014b; Cui et al., 2013; Cui et al., 2016; Liu et al., 2008; Ma et al., 2012b). These Nr losses have led to severe environmental impacts in China, including haze, acidification, eutrophication, and increased greenhouse gas emissions (Fu et al., 2007; Guo et al., 2010; Liu et al., 2013b; Qu and Kroeze, 2010; Wang et al., 2014c), which may also affect human health, for example, via toxic algae in coastal waters, acid rains and etc. (Diaz and Rosenberg, 2008; Liu et al., 2011). The Chinese population is projected to continue to grow and the food systems will likely continue to change in the coming decades (Alexandratos and Bruinsma, 2012). As a consequence, food production and import may increase. Hence nutrient losses in China's food systems to the environment may also increase as shown in previous studies (Gu et al., 2015; Ma et al., 2013a; Strokal et al., 2016a; Strokal et al., 2016b; Wang et al., 2018a). 
However, to what extent the nutrient losses will increase, is not well studied for the different socio-economic development scenarios.

Several global change scenarios have been developed during the past decades. Among them, the most widely used are the IPCC SRES scenarios (Specific Report on Emissions Scenarios), and the Millennium Ecosystem Assessment (MA) scenarios, focusing on climate change and biodiversity (Alcamo et al., 2005; Nakicenovic and Swart, 2000). More recently, Representative Concentration Pathways (RCPs) and Shared Socio-economic Pathways (SSPs) have been developed as updates of these global scenarios (Nakicenovic et al., 2014; O'Neill et al., 2014). These scenarios are meant to assess possible future climate regimes (RCPs) and also socio-economic changes (e.g., population growth, Gross Domestic Product (GDP)) (SSPs) in the global environment (O'Neill et al., 2017; O'Neill et al., 2014). The SSP storylines are largely qualitative and can be used as a basis for quantitative assessments of future global trends in human activities, and their impacts on the environment (Burek et al., 2016; Cuaresma, 2015; Popp et al., 2017; Rao et al., 2017; Valdivia et al., 2015; Van Puijenbroek et al., 2015). So far, these storylines have not been interpreted for China's food system. A good understanding of agriculture in China is important for global scenario analyses since food consumption and production change very fast in China, as we described above.

The aim of this study is to explore future trends in Nr losses from China's food system as affected by socio-economic development, taking the storylines of five SSP scenarios as a starting point. We presented a step-wise approach on how to interpret quantitatively the storylines of these scenarios for the food system of China (Figure 4.1). We first briefly described the storylines of the SSPs with a focus on the global food system (Section 4.2). Second, we interpreted these SSP storylines into qualitative storylines for China (Section 4.3). Next, we quantitatively analyzed these storylines for China's food system in 2030 and 2050 (Section 4.4). For this, we implemented the SSP scenarios in the NUFER (NUtrient Flows in Food chains, Environment and Resources use) model to project the $\mathrm{Nr}$ losses from the food system of China. Finally, we discussed the effects of the socioeconomic developments on the Nr losses from food system in the future decades in China (Sections 4.5 and 4.6). We finished this manuscript by summarizing main conclusions (Section 4.7). 


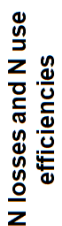

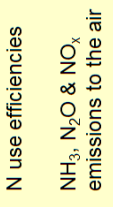

눈

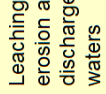

仓

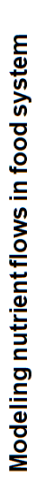

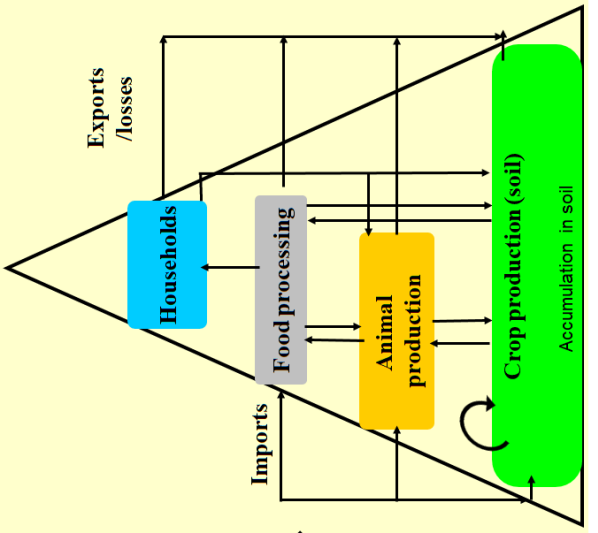

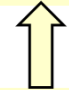

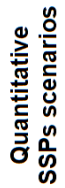

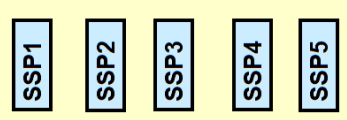

$\hat{\sqcup}$

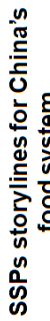
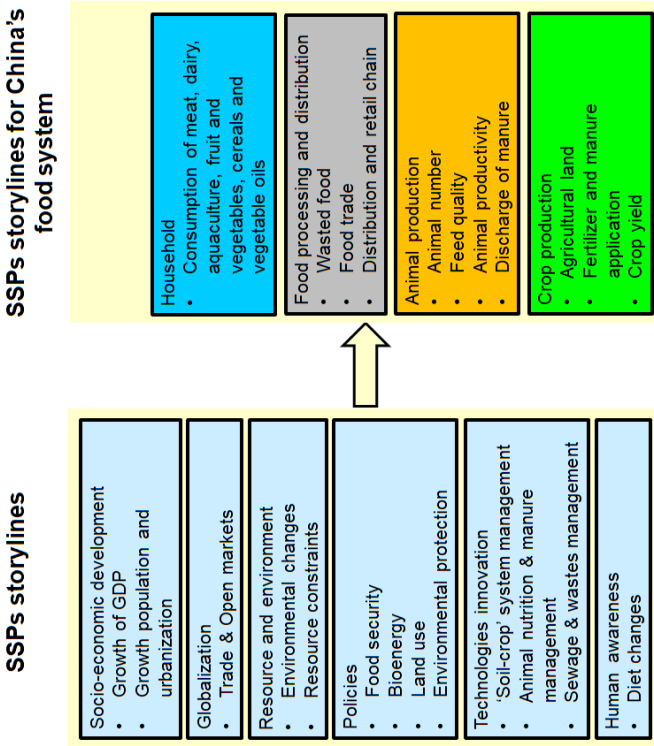

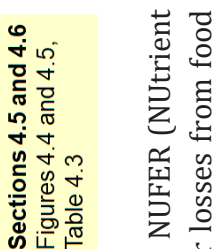

人 z

万

\% ํี

흉

氙

它它

嵌.

三

흔

के

范

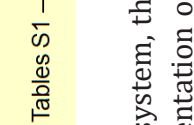

๙ क के है

宁

+

윰 苞

ஸัँ

$\hat{\square} \quad \stackrel{0}{0}$

के

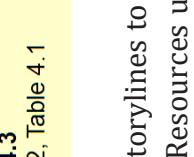

भं के क्ष

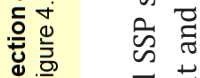

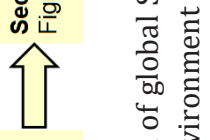

苛氙

䒕 ज宁

츨

离 ర

พับ

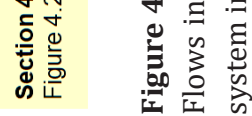




\subsection{SSP storylines as developed for global analyses}

Five storylines have been developed for SSPs at the global level. These are characterized by sustainability development (SSP1), middle of the road trends (SSP2), fragmentation (SSP3), inequality (SSP4), and conventional development (SSP5) (O'Neill et al., 2017; O'Neill et al., 2014; Team, 2011). Here we summarize the existing SSPs with a focus on future food production and consumption (Figure 4.1).

SSP1 assumes a dramatic (but gradual) shift towards sustainability, with a focus on equity, low population growth, moderate international trade, efficient use of resources, a heavy reliance on renewables, high technical solutions to pollution, and environmental policies for local and global issues. The food system can be considered sustainable, as reflected by improvements in agricultural productivity and a high preference for low-meat diets (Figure 4.2).

$\underline{\text { SSP2 }}$ assumes a moderate and diverse progress towards sustainability and economic development, with political stability for most countries, a moderate global population growth, moderate international trade, some technical development, continued environmental degradation, and environmental policies for only local pollutants. The food system has moderate improvements in agricultural productivity and a medium preference for low-meat diets (Figure 4.2).

In $\underline{\text { SSP3 }}$ countries focus on achieving national energy and food security goals within their own region at the expense of strong environmental degradation, with high population growth, strongly constrained international trade, limited development of technical solutions to pollution, limited environmental policies because of low priority for environmental issues. The food system is assumed to have low productivity in agriculture and a high preference for meat-rich consumption (Figure 4.2).

SSP4 is characterized by inequalities among and within countries, with relatively high population growth in poor regions and low population growth in rich regions. There are moderate international trade and environmental improvement in rich regions and continued degradation in low-income regions. Thus environmental policies are limited to rich regions while little attention is paid to vulnerable areas or global issues. The food system also develops unequally with higher productivity in agriculture and preference for low-meat diets in high-income regions, and low productivity in agriculture and preference for meat diets in low-income regions (Figure 4.2). 
$\underline{\text { SSP5 }}$ assumes increasingly integrated global markets and societies, with relatively low population growth, high international trade, highly managed environmental conditions, environmental policies for local issues but not for global issues. The food system develops following a conventional path with a rapid increase in productivity in agriculture and a preference for meat-rich diets (Figure 4.2).

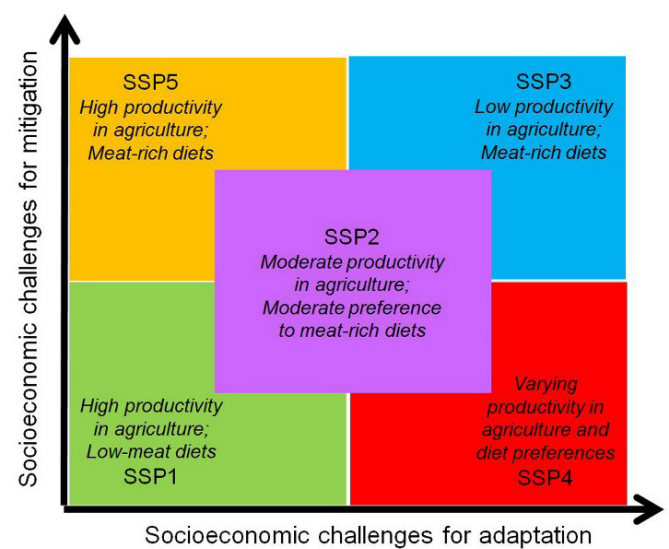

(a)

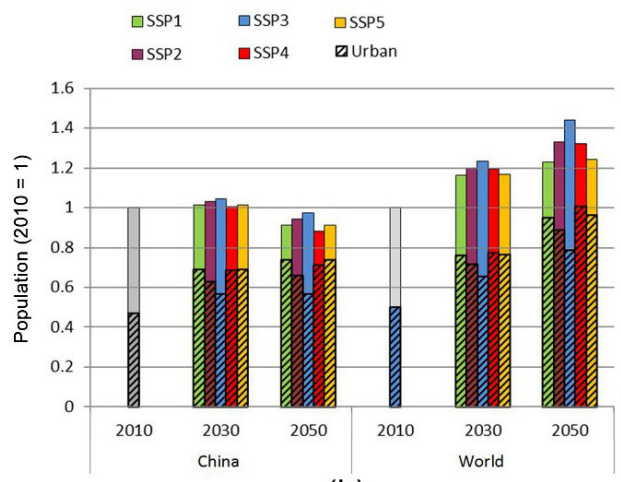

(b)

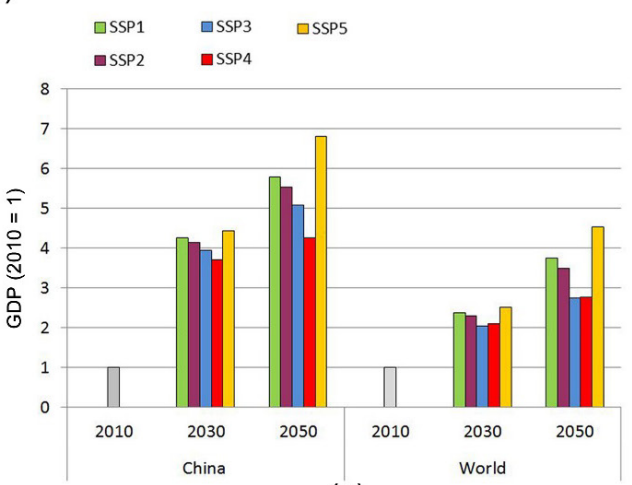

(c)

Figure 4.2 The framework of SSP scenarios with a focus on food system (a), population (b) and Gross domestic product (GDP) at purchasing power parity (ppp) (c) for China and the world in 2010,2030 and 2050. Population and GDP are shown relative to $2010(2010=1)$. The population was 6726 million people (world), and 1341 million (China) in 2010. GDP (ppp) was 65,016 billion

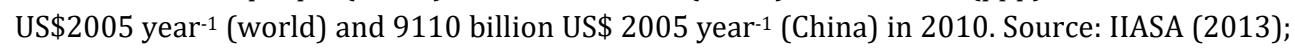
O'Neill et al. (2017). 


\subsection{Our interpretation of SSPs for China's food system}

The trends as assumed in the global SSP scenarios differ largely among and within countries. China is an interesting case as a developing country, with a rapid growing population and limited resources such as land, water, and energy. China's food system needs to feed $20 \%$ of the global population with only $7 \%$ of the global arable land (FAO, 2017a; FAO, 2017b). In general, future food systems are driven by GDP, population growth and environmental awareness. The growth rate of China's GDP is projected to be almost double that of the global average (Figure 4.2). The Chinese population may not grow as fast as the world population, but there is fast urbanization. As a result, the urban population is growing fast in China. These trends in GDP and population will drive food demand. The future level of environmental awareness will influence food losses, resource use in food production, and recycling of food waste in the food system.

In the following, we present our interpretation of the SSP storylines for China's food system based on the global SSP storylines as described above, and their implications for GDP, population and environmental awareness (Figure 4.1). We thus developed scenarios for food demand, animal product consumption, productivity in agriculture, agricultural land, food and feed imports, recycling of food waste, and mitigation techniques in food system for China (Table 4.1).

SSP1 for China: This scenario assumes a more sustainable future food system in China. Low meat-diets and a slowly growing population drive the food system. Society follows Chinese food dietary guidelines (CDGs) (Chinese Nutrition Society, 2007). These CDGs aim for healthy diets and are developed by the Chinese Nutrition Society; the recommendations are to: (i) eat a range of foods with cereals as the staple, (ii) consume adequate amounts of vegetables, fruits, and tubers, (iii) consume milk, beans, or dairy or bean products every day, (iv) consume appropriate amounts of fish, poultry, eggs, and lean meat; and (v) reduce the intake of animal fat in the diet. Based on the CDGs, the consumption of meat in SSP1 decreases and that of milk, eggs, beans and fruit increases compared to 2010 (Tables S9 - S10 in Appendix III). Due to society's high level of environmental awareness, farming practices are improving in SSP1. For example, there is a trend to produce food with less use of resources such as land, water, and nutrients. Animal production develops fast towards intensive industrial farming with high productivity. At the same time, residues, manure and food wastes from the food 
production-consumption chain are recycled in the food system under stringent and effective environmental policies. Mitigation technologies for nutrient losses, such as appropriate fertilizer and manure application techniques, manure storage and processing techniques, are applied.

SSP2 for China: This scenario assumes moderate improvements in the food system of China. Food demand increases because of population growth and rapid urbanization. The consumption of meat decreases, but not as much as in SPP1, and that of milk, eggs, beans and fruit increases. Food supply relies on both increasing local productivities with less agricultural land and imports of food and feeds from other countries. Animal production develops towards intensive industrial farming in China, but not as fast as in SSP1. Technologies for increasing food productivities, recycling residues, manure, and food waste, and mitigating nutrient losses are applied in regions where the food systems are more developed, but not in all regions in China.

SSP3 for China: This scenario assumes a future in which food security and self-sufficiency are the first priority. Due to the fast population growth and a preference for meat, food demand in China increases faster than in other SSP scenarios. All food is produced in China. Imports of feed and food are strongly constrained. In crop production, increasing amounts of synthetic fertilizer are used. Animal production takes place in both intensive industrial farming and traditional small-scale farming. There are limited environmental policies and technologies for recycling crop residues, animal manure and food wastes, and mitigating nutrient losses in the food system of China.

SSP4 for China: This scenario is characterized by inequalities in China. Food demand increases, but not in all parts of China. It is lower in low-income regions, and medium in high-income regions. In this scenario, more and more food is from intensive industrial farming with a high-income. Animal production develops fast towards intensive industrial farming in high-income regions, while in low-income regions traditional small-scale animal farms dominate. Moderate imports of feed and food also contribute to feeding the relatively large population. In high-income regions there is some recycling of crop residues, animal manure and food wastes in the food system, with relatively strict environmental policies. Very limited attention is paid to environmental improvement in low-income regions. 
SSP5 for China: This scenario assumes a conventional food system in the future. In SSP5, food demand and consumption increase because of increasing preference for meat-rich diets. The population growth is somewhat lower than in other SSPs. Food is produced with increasing productivity on less agricultural land. In addition, there is import of food and feed. Animal production develops fast towards intensive industrial farming with high productivity. Technologies are developed for recycling crop residues, animal manure and food waste in the food system within the region. 


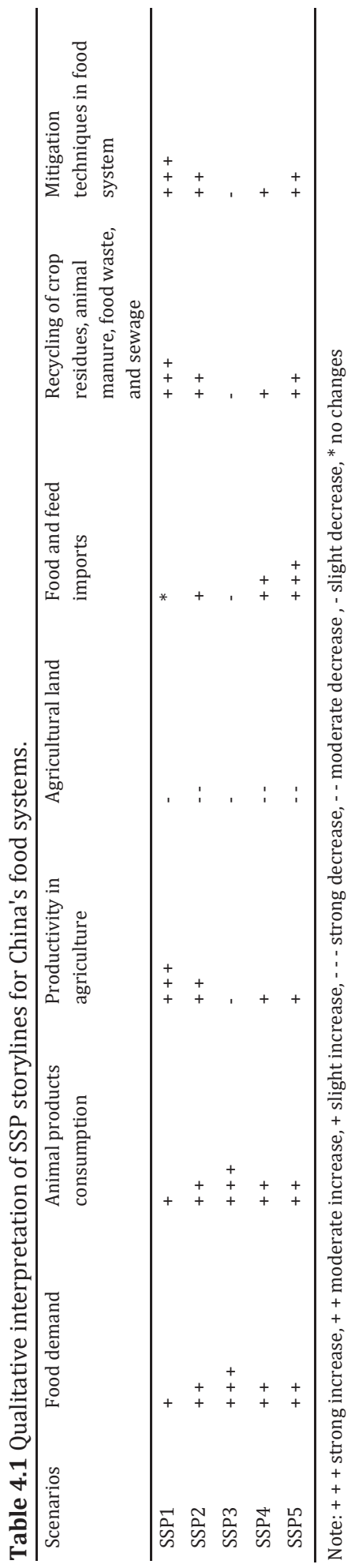




\subsection{Calculating Nr losses by NUFER}

We used the NUFER model to analyze future trends in Nr losses from China's food system under the socio-economic development as described in the SSP storylines (Figure 4.1). This model has been widely used to analyze the nutrient flows along the food productionconsumption chain in China (Bai et al., 2014b; Bai et al., 2013; Hou et al., 2013; Ma et al., 2010; Ma et al., 2012b; Ma et al., 2013a; Ma et al., 2013b). China's food system is perceived as a food production-consumption chain in NUFER (Figure 4.3). This chain consists of crop production, animal production, food processing, and household consumption. NUFER is a visual and simple tool to analyze N and P flows in the food system (Figure 4.3) (Ma et al., 2012b). N and P flows are quantified by NUFER at regional (provincial and county) and national scales for past (1980-2010), current (2013) and future years (2030 and 2050). Based on the $\mathrm{N}$ and $\mathrm{P}$ flows, $\mathrm{N}$ and $\mathrm{P}$ losses from the food production chain can be calculated using NUFER.

In this study, the year 2010 was used as a reference. The five SSP scenarios were implemented in NUFER for 2030 and 2050. This quantitative scenario analysis follows from the SSP storylines for China's food system as presented in Section 4.3 (Table 4.1). We used information on the food system in 2010 (Ma et al., 2012b; MOA, 2011), expert knowledge and some current food production and environmental policies (Chinese Nutrition Society, 2007; MOA, 2015c; Zheng, 2013). The quantitative SSP scenarios include the changes in the model inputs for crop production (e.g., crop yield, synthetic fertilizer application) (Tables S1 and S2 in Appendix III), animal production (e.g., animal number, industrialization of animal production, manure management, Figure 4.3, Tables S3 - S5 in Appendix III), food processing (e.g., treatment and recycle of processing waste, Figure 4.3), and household consumption (e.g., treatment and recycle of food waste, import of food, Figure 4.3, Tables S6 - S10 in Appendix III). Table 4.2 summarizes the model inputs for 2030 and 2050 that are included in Appendix III.

We calculated Nr losses from the food system for 2010, 2030 and 2050. The Nr losses to the air include emissions of ammonia $\left(\mathrm{NH}_{3}\right)$, nitrous oxide $\left(\mathrm{N}_{2} \mathrm{O}\right)$ and nitrogen oxides $\left(\mathrm{NO}_{\mathrm{x}}\right) . \mathrm{Nr}$ losses to water include leaching, runoff, erosion, and discharge of animal manure, food waste, human manure and kitchen residues to rivers. In NUFER the losses of $\mathrm{Nr}$ are quantified based on either $\mathrm{N}$-source specific fractions (e.g., fraction of $\mathrm{NH}_{3}$ losses from animal manure) or soil, geomorphology and climate-specific fractions (e.g., fraction 
of $\mathrm{N}$ leaching in soil) (Ma et al., 2010). Most of these fractions (e.g., the fraction for the discharge of animal manure) are a function of nutrient management. Therefore, the fractions vary among SSP scenarios (see Figure 4.3). A detailed description of calculations in NUFER is presented in Ma et al. (2010) and Ma et al. (2012b).

We also calculated the $\mathrm{N}$ use efficiencies of crop production, animal production, food processing, and the whole food production-consumption chain in China using NUFER. N use efficiency is calculated by dividing $\mathrm{N}$ contents in the main products (e.g., crops, meat, milk, egg and etc.) by $\mathrm{N}$ inputs to food system compartments (see the equation in Box 4.1). The detailed calculation method is available in earlier studies (Ma et al., 2012b; Wang et al., 2018a).

Box 4.1 Equation to quantify nitrogen use efficiency (NUE) in the food system by the NUFER (NUtrient Flows in Food chains, Environment and Resources use) model. The equation is based on Ma et al. (2012b) and Wang et al. (2018a).

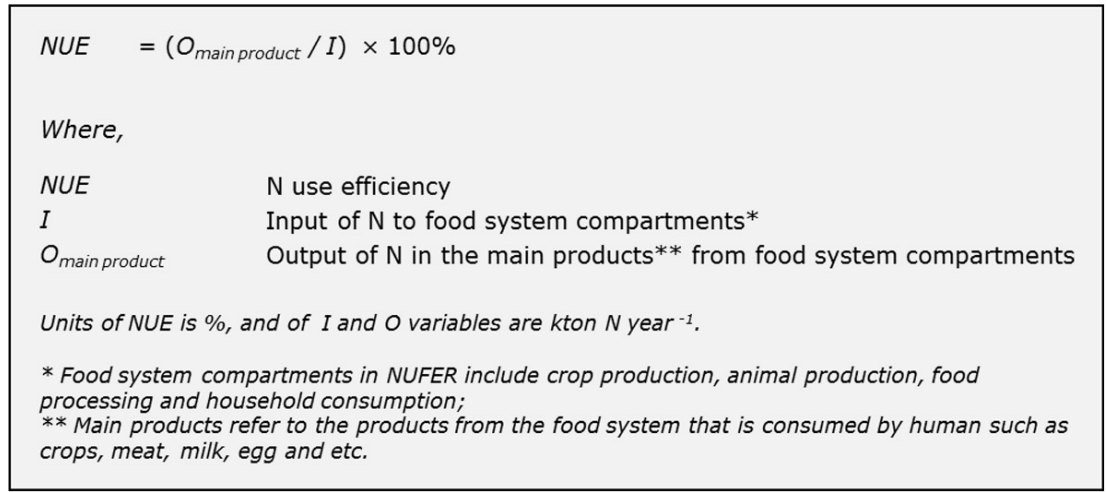

Table 4.2 Model inputs for NUFER (NUtrient Flows in Food chains, Environment and Resources use) in 2010, 2030 and 2050 in five SSP scenarios. N refers to nitrogen.

\begin{tabular}{ll}
\hline Model inputs of & Numbers are available in \\
\hline The emission factors for N losses to waters and the air & Figure 4.3 \\
Application of synthetic fertilizers & Table S1 in Appendix III \\
Yield of crops & Table S2 in Appendix III \\
Animal numbers & Table S3 in Appendix III \\
Percentage of intensive-industrial farming & Table S4 in Appendix III \\
Manure nitrogen excretion & Table S5 in Appendix III \\
Population in urban and rural area & Table S6 in Appendix III \\
Import of crop food & Table S7 in Appendix III \\
Import of animal food & Table S8 in Appendix III \\
Food consumption of crop products in urban and rural area & Table S9 in Appendix III \\
Food consumption of animal products in urban and rural area & Table S10 in Appendix III \\
\hline
\end{tabular}




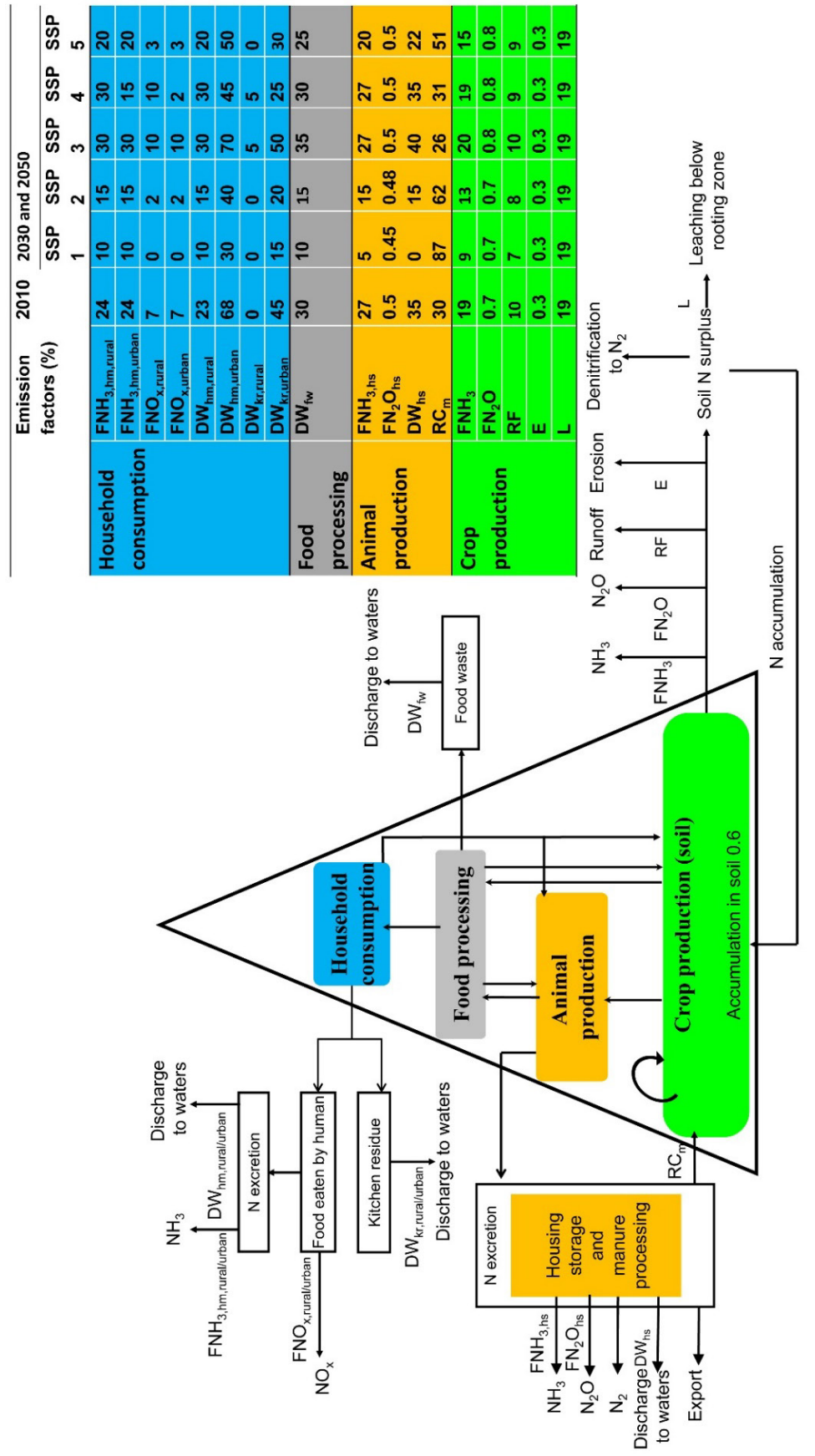

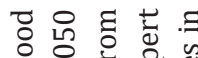

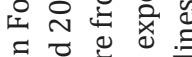
$\exists$ 范菏

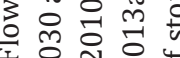

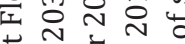

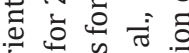
E 芯焉要

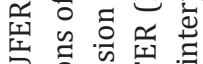
完记要 要

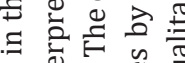

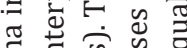

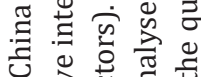
记陆

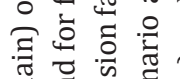

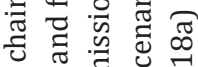
¿ 0 ह छ थ 융

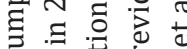
ป क :

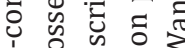
흥음 임 乙

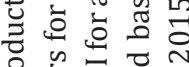

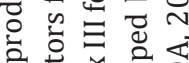

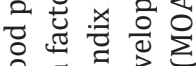

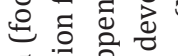

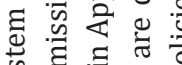
की एव के जै जि ¿ $\frac{0}{0}$ $\stackrel{0}{2} \stackrel{0}{0}$ Ð ¿ छ ⿻ 丨

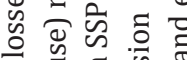
ż

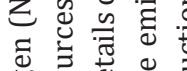
๑ొ

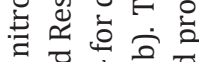

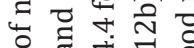
釈 站泀 m.

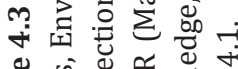

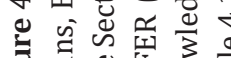

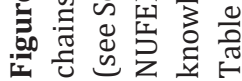




\subsection{Future $\mathrm{Nr}$ losses}

Future Nr losses differ largely among scenarios. Nr losses from the whole food system of China to the environment are projected to increase in SSP3 and SSP4 (Figures 4.4 and 4.5). The peak in 2030 can be explained by a peak in the population. In 2050, the total Nr losses to the air are 45\% higher than in 2010 in SSP3, and 16\% higher in SSP4. Total Nr losses to waters are projected to increase by $9 \mathrm{Tg}$ in SSP3, and by $2 \mathrm{Tg}$ in SSP4 between 2010 and 2050. Future trends in Nr losses from crop production, animal production, food processing and household consumption in SSP3 and SSP4 are similar to the trends for the whole system (Figures 4.4 and 4.5). The Nr losses from all four compartments increase between 2010 and 2050 in SSP3 and SSP4 except for the household compartment. $\mathrm{Nr}$ losses from the household compartment increase in SSP3 and decrease in SSP4 between 2010 and 2050. The relative share of the four compartments in total $\mathrm{Nr}$ losses changes over time (Figures 4.4 and 4.5). This can be explained by differences in $\mathrm{N}$ use, nutrient management and treatment. For example, in $205040 \%$ of total $\mathrm{Nr}$ losses to the air and $50 \%$ of total $\mathrm{Nr}$ losses to waters are from animal production in SSP3. This is much higher than in 2010 when $31 \%$ of total $\mathrm{Nr}$ losses to the air and $37 \%$ of total $\mathrm{Nr}$ losses to waters were from animal production. This is because of the poor recycling of animal manure, and poor control of $\mathrm{NH}_{3}$ and $\mathrm{N}_{2} \mathrm{O}$ emissions during storage of animal manure in SSP3 (Figure 4.3, Table 4.1).

In SSP5 food production is assumed to develop following a conventional pathway. As a result, Nr losses to the air and waters are slightly lower than in 2010 (Figures 4.4 and 4.5). In 2050, the total losses of Nr to the air in SSP5 are 15\% lower, and to waters are $10 \%$ lower than in 2010. The share of the four compartments in total Nr losses does not change much over time in this scenario. In $2050 \mathrm{Nr}$ losses to the air are dominated by $\mathrm{NH}_{3}$ emission from crop production (53\% of total $\mathrm{Nr}$ losses to the air) and $\mathrm{NH}_{3}$ emission from animal production (36\% of total $\mathrm{Nr}$ losses to the air) in SSP5. The major source of $\mathrm{Nr}$ losses to waters is the discharge of animal manure to waters (36\% of total $\mathrm{Nr}$ losses to waters), runoff (28\% of total $\mathrm{Nr}$ losses to waters) and leaching (18\% of total $\mathrm{Nr}$ losses to waters) of $\mathrm{N}$ to waters from crop production in SSP5 in 2050.

SSP1 and SSP2 are more sustainable scenarios, with relatively low $\mathrm{Nr}$ losses to the environment while ensuring food security (Figures 4.4 and 4.5). The total Nr losses to the air in 2050 in SSP1 are projected to be 72\% lower, and in SSP2 34\% lower than in 2010. 
In SSP1 the total Nr losses to waters in 2050 are $10 \mathrm{Tg}$ lower than in 2010, and in SSP2 4 Tg lower. The Nr losses from all four compartments decrease between 2010 and 2050 in SSP1 and SSP2 (Figures 4.4 and 4.5). The share of the four compartments in total Nr losses changes over time in SSP1 and SSP2 (Figures 4.4 and 4.5). For example, in 2050 22\% of total $\mathrm{Nr}$ losses to the air and $0 \%$ of total $\mathrm{Nr}$ losses to waters are from animal production in SSP1. This is much lower than in 2010 when $31 \%$ of total $\mathrm{Nr}$ losses to the air and 37\% of total $\mathrm{Nr}$ losses to waters were from animal production. This change is resulted from the improvements in the recycling of animal manure, and mitigating $\mathrm{NH}_{3}$ and $\mathrm{N}_{2} \mathrm{O}$ emissions during the storage of animal manure in SSP1 (Figures 4.2 and 4.3).

The Nr losses from food systems are the net effect of total $\mathrm{N}$ inputs, the $\mathrm{N}$ use efficiencies, and the management of $\mathrm{N}$ in the food system (e.g., recycling of $\mathrm{N}$, and mitigation of $\mathrm{Nr}$ losses). The $\mathrm{N}$ use efficiencies are, therefore, calculated for China's food system (Table 4.3). Results show that the $\mathrm{N}$ use efficiencies of the whole food production-consumption chain is low in 2010 (9\%) and varies from 6 to 21\% among SSP scenarios in 2050. The N use efficiencies increase from 9 to $21 \%$ in SSP1, and from 9 to $12 \%$ in SSP2. In SSP3, however, $\mathrm{N}$ use efficiencies decrease from 9 to 6\% between 2010 and 2050, and in SSP4 and SSP5 they stabilize at their low levels of 2010. The trends in NUEs of crop production and animal production in SSPs between 2010 and 2050 are similar to the trends in NUEs of the whole food production-consumption chain. For food processing, however, we calculate that the high $\mathrm{N}$ use efficiency (76\% in 2010) do not change over time. Our results indicate that the increases in Nr losses in SSP3 and SSP4 are related to the high inputs of $\mathrm{N}$ to food production, the low $\mathrm{N}$ use efficiencies, the low recycling of $\mathrm{N}$, and low mitigation of Nr losses (Figure 4.3, Tables S1 - S10 in Appendix III, see Table 4.2 for the summary of the information that is included in Tables S1 - S10). The decreases in Nr losses in SSP1, SSP2 and SSP5 result from the relatively low input of $N$ to food production, high $\mathrm{N}$ use efficiencies, high recycling of $\mathrm{N}$, and high mitigation of Nr losses (Figure 4.3, Tables S1 S10 in Appendix III, see Table 4.2 for the summary of the information that is included in Tables S1 - S10).

Our quantitative interpretations of the five SSPs may help policymakers to decide on socio-economic and environmental policies, and technologies for a sustainable future food system in China. For instance, in SSP1 and SSP2 China may continue developing fast economically (high GDP in 2050 as indicated in Figure 4.2), but with low population 
growth and low environmental pollution (low Nr losses in 2050 as indicated in Figures 4.4 and 4.5). The low losses of Nr in SSP1 and SSP2 scenarios follow from improvements in the food system such as increased productivity in agriculture, and recycling of waste in the food production-consumption chain. In SSP5, the economy may develop faster than in SSP1 and SSP2 as indicated by the higher GDP in 2030 and 2050 (Figure 4.2). However, the Nr losses from the food system are also higher than in SSP1 and SSP2. If China would develop as described in SSP5, additional environmental policies would be needed to limit the pollution. For instance, policies aiming at higher recycling of waste in the food system, and technologies for pollution mitigation that were included in SSP1 and SSP2 could be considered to reduce Nr losses to the environment. In SSP3 and SSP4 the economy does not develop as fast as in the other SSPs scenarios (see GDP in Figure 4.2), while the $\mathrm{Nr}$ losses from the food system are much higher than in the other SSPs. To avoid this, additional socio-economic and environmental policies would be needed in these two futures. 


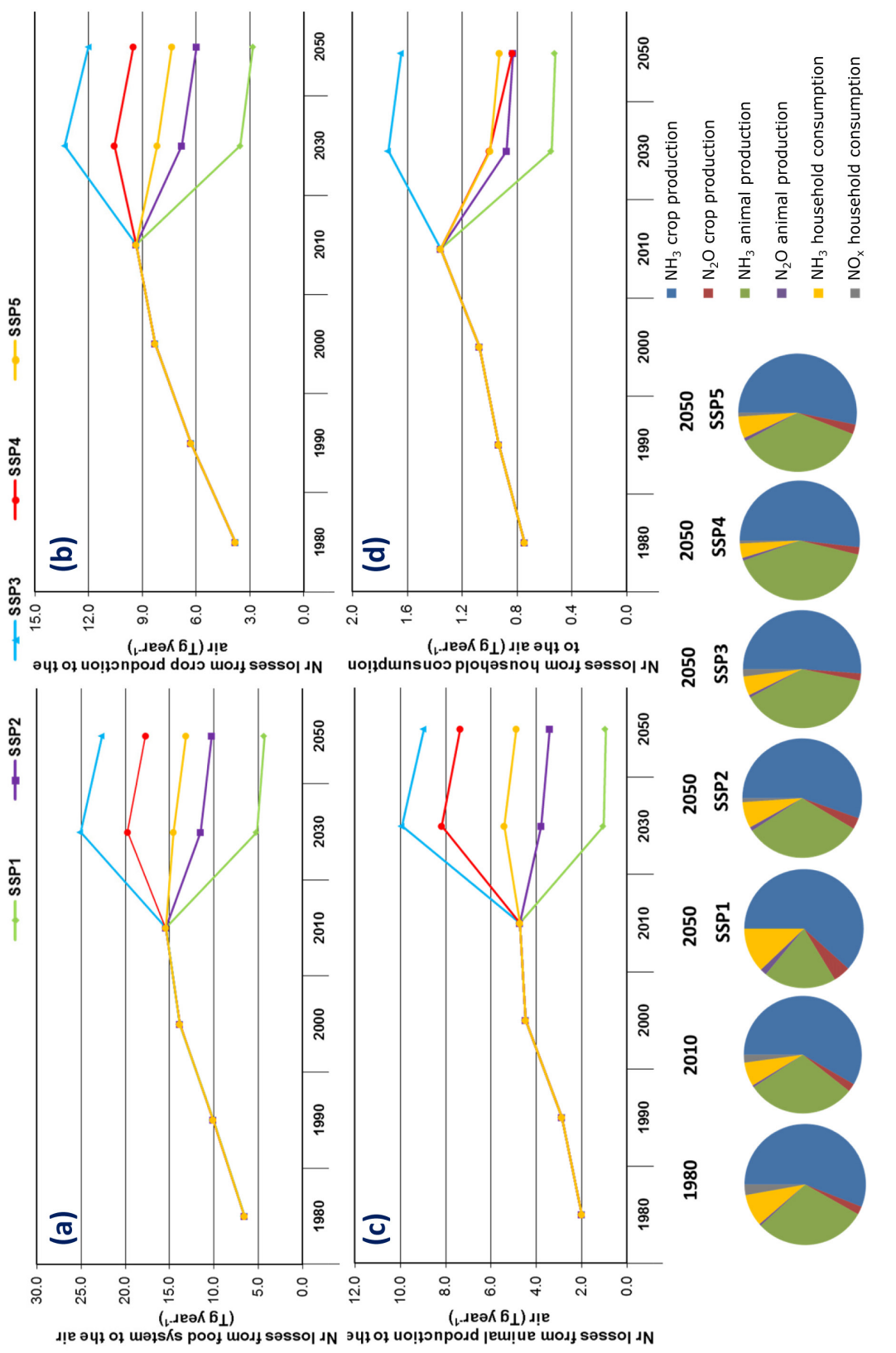

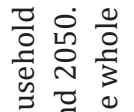

芩芯

휘요

웡 d

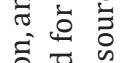

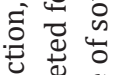

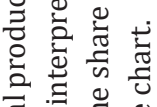

䒕芯氙.气

तี

త

竞

ड़े के

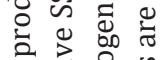

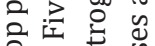

○ें

อ 유을

घี ত

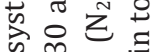

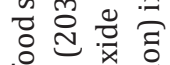

잉

品壱告

记

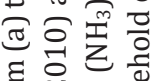

घี จ

离䓠

क

๖

00

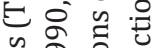

क्षे के

○े की

艺氖它

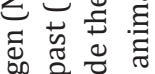

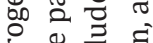

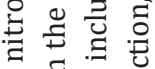

$\stackrel{\Xi}{\Xi}$.

元焉

픈

○

†.

+ 完它

衰言言善 


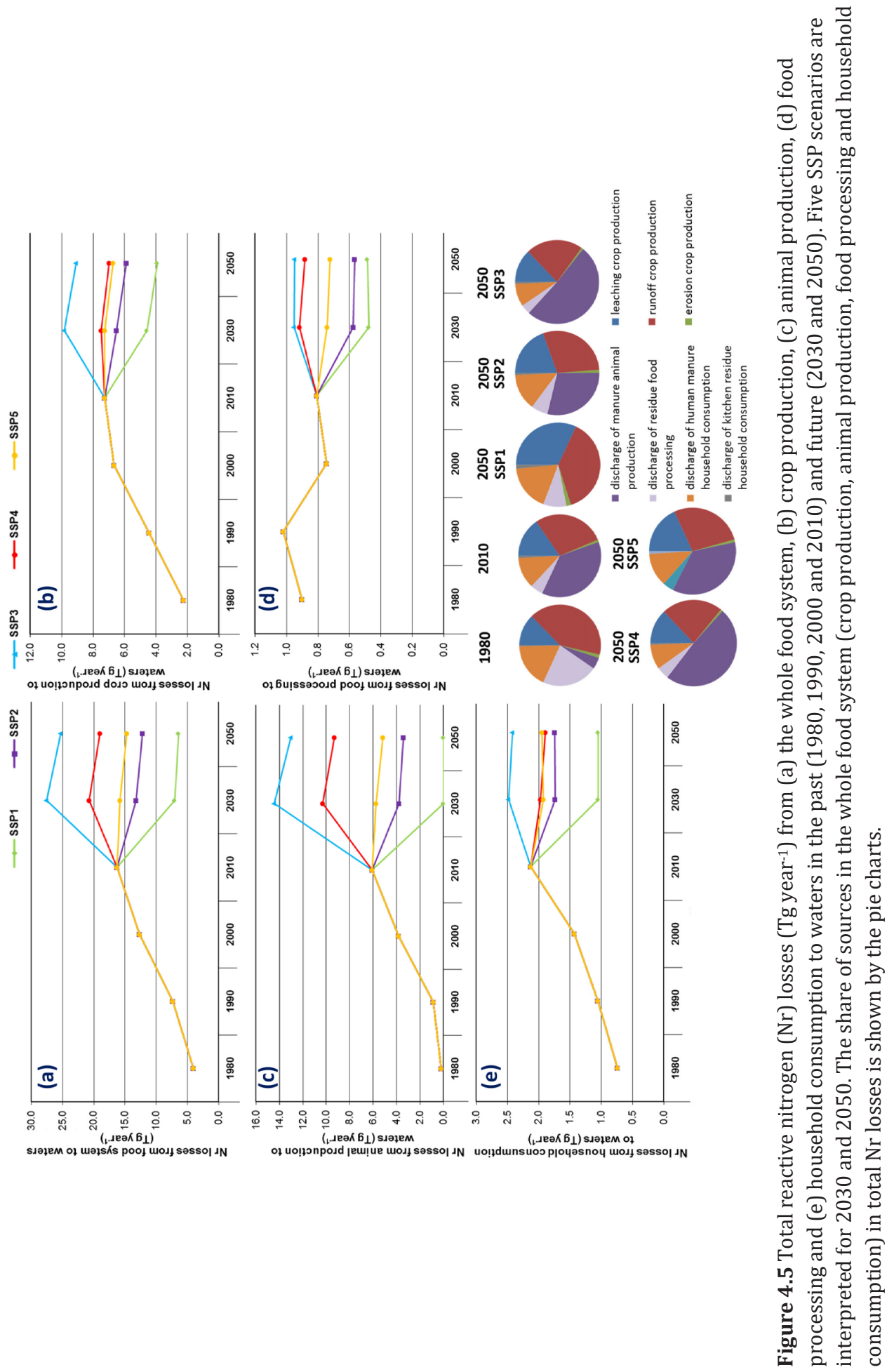


Table 4.3 Nitrogen use efficiencies of crop production (NUEc), animal production (NUEa), food processing (NUEfp), and the whole food system (NUEf) in 2010 and 2050. Five SSP scenarios are interpreted for 2050. The equation for calculating NUEs is available in Box 4.1.

\begin{tabular}{ll|lllll}
\hline & 2010 & 2050 & & & & \\
& & SSP1 & SSP2 & SSP3 & SSP4 & SSP5 \\
\hline NUEc & 29 & 36 & 34 & 27 & 30 & 31 \\
NUEa & 13 & 14 & 13 & 10 & 11 & 12 \\
NUEfp & 76 & 75 & 76 & 75 & 77 & 77 \\
NUEf & 9 & 21 & 12 & 6 & 8 & 9 \\
\hline
\end{tabular}

\subsection{Comparison with earlier scenario studies}

Scenario analysis is commonly used to explore future changes in the environment, and to assess options for reducing the negative environmental impacts from the food system. Earlier studies mainly differ from our study in the methods to develop scenarios. Earlier studies either developed scenarios from population and food demand prospects (Gu et al., 2015; Ma et al., 2013a), or from existing quantitative interpretation of scenarios (MA scenarios in Qu and Kroeze, 2012; Strokal et al., 2017; and FAO projections in Wang et al., 2018a) (Table S12 in Appendix III). Some studies incorporate the current environmental policies regarding food production-consumption (Oenema et al., 2009), and some studies do not (Table S12 in Appendix III).

Here we use the recent global scenarios - SSPs as the basic storylines, and interpreted specific scenarios for food systems in China. This was not done by other studies so far. By using the SSPs as a basis for storylines, we also incorporated the current and possible future environmental policies for the food system in our scenarios. In addition, the SSP scenarios were interpreted here specifically for China's food system, while other studies mainly focus on the global levels or for other systems (Arnell and Lloyd-Hughes, 2014; Burek et al., 2016; Popp et al., 2017; Rao et al., 2017; Valdivia et al., 2015; Van Puijenbroek et al., 2015; Wiebe et al., 2015). For example, the representative agricultural pathways and scenarios (RAPs) were developed for the global agricultural system (Rosenzweig et al., 2013; Valdivia et al., 2015). Arnell and Lloyd-Hughes (2014); Burek et al. (2016) implemented the SSPs in climate models to assess the global future water situation such as water demand and water scarcity. Future global air pollution (Rao et al., 2017), sanitation and wastewater flows (Van Puijenbroek et al., 2015) were also studied using the SSPs. 
Similar to all other scenario studies, there are uncertainties in the model, and in the assumptions for future trends. The uncertainties in the NUFER model relate to the model inputs, the coefficients that are used in the model and model structure. The model inputs in 2010 are taken from the Chinese statistical yearbook, which we believe is the most reliable data source in China. The coefficient refers to the nutrient content fractions (e.g., $\mathrm{N}$ content in crops and animals), and the nutrient emission factors that are used in the model. These factors in 2010 are mostly taken from other peer-reviewed papers and results of interviewing farmers in China as it is described in Ma et al. (2010). There are also uncertainties related to the assumptions about possible development pathways of the food system in our SSP scenarios. For example, in SSP1 it is assumed that animal manure is not discharged from the production sector to rivers at all. This is an ideal state that may be difficult to reach in the future. Despite these uncertainties, we believe that our study gives useful insights in possible future trends in $\mathrm{Nr}$ losses under different socioeconomic pathways. It can be used for policy development aimed at reducing the negative environmental impacts (Nr losses) of food production and consumption. The step-wise approach that we developed to interpret qualitative storylines into quantitative scenarios can be used as an example for other studies or other world regions. In addition, our qualitative and quantitative interpretations of the SSPs can be useful for other studies to explore possible future changes in the food system of China.

\subsection{Conclusion}

We explored future trends in losses of $\mathrm{Nr}$ from China's food system based on the SSP storylines. We interpreted the SSP storylines into qualitative storylines for China's food system. Five quantitative SSP scenarios for 2030 and 2050 were interpreted based on the qualitative storylines, and were implemented in the NUFER model.

Our analysis shows that $\mathrm{Nr}$ losses from food system of China vary among SSPs. The $\mathrm{Nr}$ losses to the environment will likely increase in the coming decades under the socioeconomic development in SSP3 and SSP4. In SSP3, the total Nr losses to the air and waters are projected to increase by $45 \%$ and $55 \%$ between 2010 and 2050, respectively. This leads to a future with higher potentials for pollution than today. The food system of China in SSP5 takes a more conventional path under which the Nr losses are slightly lower than today (15\% lower for air and 10\% lower for waters). Even lower $\mathrm{Nr}$ losses are projected for SSP1 and SSP2. The total Nr losses to the air in 2050 in SSP1 are reduced by more than 
two-thirds relative to 2010, and in SSP2 by one third. The total Nr losses to waters are projected to have a $60 \%$ reduction in SSP1, and a 25\% reduction in SSP2 between 2010 and 2050.

Our study shows how future trends in $\mathrm{Nr}$ losses may be affected by socio-economic development and environmental policies. The scenarios with high $\mathrm{Nr}$ losses under fast (SSP5) or slow economic (SSP3 and SSP4) development show that improvement in both socio-economic and environmental policies are important for China's food system. The scenario analysis also reveals potential pathways to ensure food security and fast economic development with relatively low environmental pollution as indicated by the lower Nr losses in SSP1 and SSP2 scenarios. These low losses follow from improvements in food production and consumption such as increased productivity in agriculture, and recycling of waste in the food production-consumption chain. Our study is the first to interpret SSPs for China's food system. The qualitative and quantitative SSP scenarios that are interpreted in this study can provide a good basis for other studies aiming to explore future trends in the food system of China. The step-wise approach that we developed to interpret quantitatively the SSPs may also be used for scenario analysis of food system in other world regions.

\section{Acknowledgments}

We acknowledge The National Key Research and Development Program of China (2016YFD0800106), Wageningen Institute for Environment and Climate Research (WIMEK) of Wageningen University \& Research, and Netherlands Organization for Scientific Research (NWO) for financial support. We also acknowledge the Chinese National Basic Research Program (2015CB150405), the National Natural Science Foundation of China (31572210), President's International Fellowship Initiative, PIFI of the Chinese Academy of Science (2015VEA025), the Hundred Talent Program of the Chinese Academy of Science for supporting this research. 



\title{
Chapter 5. Global Change Can Make Coastal Eutrophication Control in China More Difficult
}

\begin{abstract}
Fast socio-economic development in agriculture and urbanization resulted in increasing nutrient export by rivers, causing coastal eutrophication in China. In addition, climate change may affect hydrology, and as a result, affect nutrient flows from land to sea. This study aims at a better understanding of how future socio-economic and climatic changes may affect coastal eutrophication in China. We modeled river export of total dissolved nitrogen (TDN) and phosphorus (TDP) in 2050 for six scenarios combining Socioeconomic Pathways (SSPs) and Representative Concentration Pathways (RCPs). We used the newly developed MARINA 2.0 (Model to Assess River Inputs of Nutrients to seAs) model. We found that global change can make coastal eutrophication control in China more difficult. In 2050 coastal waters may be considerably more polluted or considerably cleaner than today depending on the SSP-RCP scenarios. By 2050, river export of TDN and TDP is 52\% and 56\% higher than in 2012, respectively, in SSP3-RCP8.5 (assuming large challenges for sustainable socio-economic development, and severe climate change). In contrast, river export of nutrients could be 56\% (TDN) and 85\% (TDP) lower in 2050 than in 2012 in SSP1-RCP2.6 (assuming sustainable socio-economic development, and low climate change). Climate change alone may increase river export of nutrients considerably through hydrology: We calculate $24 \%$ higher river export of TDN and $16 \%$ higher TDP for the SSP2 scenario assuming severe climate change compared to the same scenario with low climate change (SSP2-RCP8.5 versus SSP2-RCP2.6). Policies and relevant technologies combining improved nutrient management and climate mitigation may help to improve water quality in rivers and coastal waters of China.
\end{abstract}

Submitted as:

Wang M., Kroeze C., Strokal M., Van Vliet M.T.H., Ma L. Global change can make coastal eutrophication control in China more difficult. Submitted for publication in Earth's Future (this chapter is based on the manuscript that was resubmitted after positive review). 


\subsection{Introduction}

Socio-economic changes associated with population growth, urbanization, food production and waste management alter biogeochemical cycles (Billen et al., 2010; Bouwman et al., 2009; Van Puijenbroek et al., 2019). In many world regions, this has resulted in increasing nutrient inputs to rivers. These nutrients are transported by rivers to seas, causing coastal eutrophication (Amin et al., 2017; McCrackin et al., 2015; Pedde et al., 2017; Seitzinger et al., 2010).

China experienced rapid socio-economic development in the last decades (Bai et al., 2014a). Urbanization and food production have caused increasing nutrient inputs to water systems (Ma et al., 2014b; Yu et al., 2019). The use of synthetic fertilizers in China increased by almost 5 fold from 13 Mton in 1980 to 58 Mton in 2012 (NBSC, 2013). However, typically less than half of these nutrients are used by crops (Ma et al., 2012b; Wang et al., 2018a). The remainder is lost to air or waters. In 2012, China produced in total 68,000 Mton of wastewater (MEP, 2012). Only 60-82\% of this was treated before being discharged to rivers (MOHURD, 2012). As a result, rivers transport increasing amounts of nutrients to coastal waters, causing coastal eutrophication and harmful algal blooms (Dai et al., 2010; Huan et al., 2016; Zhou et al., 2018). Such blooms occurred approximately 500 times in coastal areas of China between 2006 and 2012 (SOA, 2012). The area affected by coastal eutrophication in China has been increasing to be $98,000 \mathrm{~km}^{2}$ in 2012 (SOA, 2012).

There may be more coastal eutrophication in the future as a result of socio-economic development (Crespo Cuaresma, 2017). In addition, global climate change may affect hydrology, and as a result river discharge. This may, in turn, affect nutrient levels in rivers (Han et al., 2009; Howarth et al., 2006; Rabalais et al., 2009; Sinha et al., 2017). For example, Sinha et al. (2017) concluded that climate change-induced changes in precipitation alone may substantially increase river export of nitrogen in Eastern China by the end of the century. To what extent, however, is unclear. A better understanding of the combined impacts of socio-economic and climate change on future river export of nutrients will contribute to the formulation of effective policies for improving water quality in China, and other rapidly developing world regions.

Modeling studies exist to quantify past trends in river export of nutrients to seas in China (Liu et al., 2019; Liu et al., 2018a; Qu and Kroeze, 2010; Strokal et al., 2016a; UNEP, 2016b). 
A few of these studies (Qu and Kroeze, 2010; Strokal et al., 2016a; Strokal et al., 2017) explored future trends, and focused on the impacts of socio-economic change based on the old Millennium Ecosystem Assessment (MEA) scenarios (Alcamo et al., 2005). So far, the Shared Socio-economic Pathways (SSPs) and Representative Concentration Pathways (RCPs) have not yet been used to assess future nutrient export by rivers in China. SSPs are global storylines describing future socio-economic development such as changes in population, urbanization and gross domestic product (O'Neill et al., 2014). RCPs are four pathways covering a range of radiative forcing values of the year 2100 from 2.6 to 8.5 $\mathrm{W} / \mathrm{m}^{2}$ (Van Vuuren et al., 2011). Such an assessment is important, since socio-economic development and climate change together may strongly impact river export of nutrients to coastal waters (Sinha et al., 2019).

This study, therefore, aims at a better understanding of how future socio-economic and climatic changes may affect coastal eutrophication through future river export of total dissolved nitrogen (TDN) and phosphorus (TDP) by Chinese rivers at the sub-basin scale, and by source. To this end, we applied the newly developed MARINA 2.0 (Model to Assess River Inputs of Nutrients to seAs) model for 12 Chinese rivers and their sub-basins. For 2050, six scenarios combining the SSPs and RCPs were explored. We analyzed hotspots of $\mathrm{N}$ and P losses to rivers in 2012 and 2050 to better understand the impacts of socioeconomic change on river export of nutrients. We also analyzed the potential impacts of climate change on river export of nutrients. Finally, we discussed opportunities for improving water quality in Chinese rivers and coastal waters. This study will contribute to a better understanding of future coastal eutrophication as affected by global change in China. The MARINA 2.0 model and the scenario approach have potential applications to other regions of the world.

\subsection{Method}

\subsubsection{The study area}

We quantified river export of total dissolved nitrogen (TDN) and phosphorus (TDP) for 12 Chinese rivers draining into four seas: Bohai Gulf, Yellow Sea, East China Sea and South China Sea (Figure 5.1). The drainage area of these rivers covers $40 \%$ of China's land (around 4 million $\mathrm{km}^{-2}$ ). The drainage basins of Yellow (1.92 million $\mathrm{km}^{-2}$ ), Yangtze (0.76 million $\mathrm{km}^{-2}$ ) and Pearl (0.43 million $\mathrm{km}^{-2}$ ) were divided into upstream, middle-steam and 
downstream sub-basins largely following Strokal et al. (2016a) (see Figure 5.2, and

Figures A.3-A.5 in Appendix IV for names and locations of sub-basins).

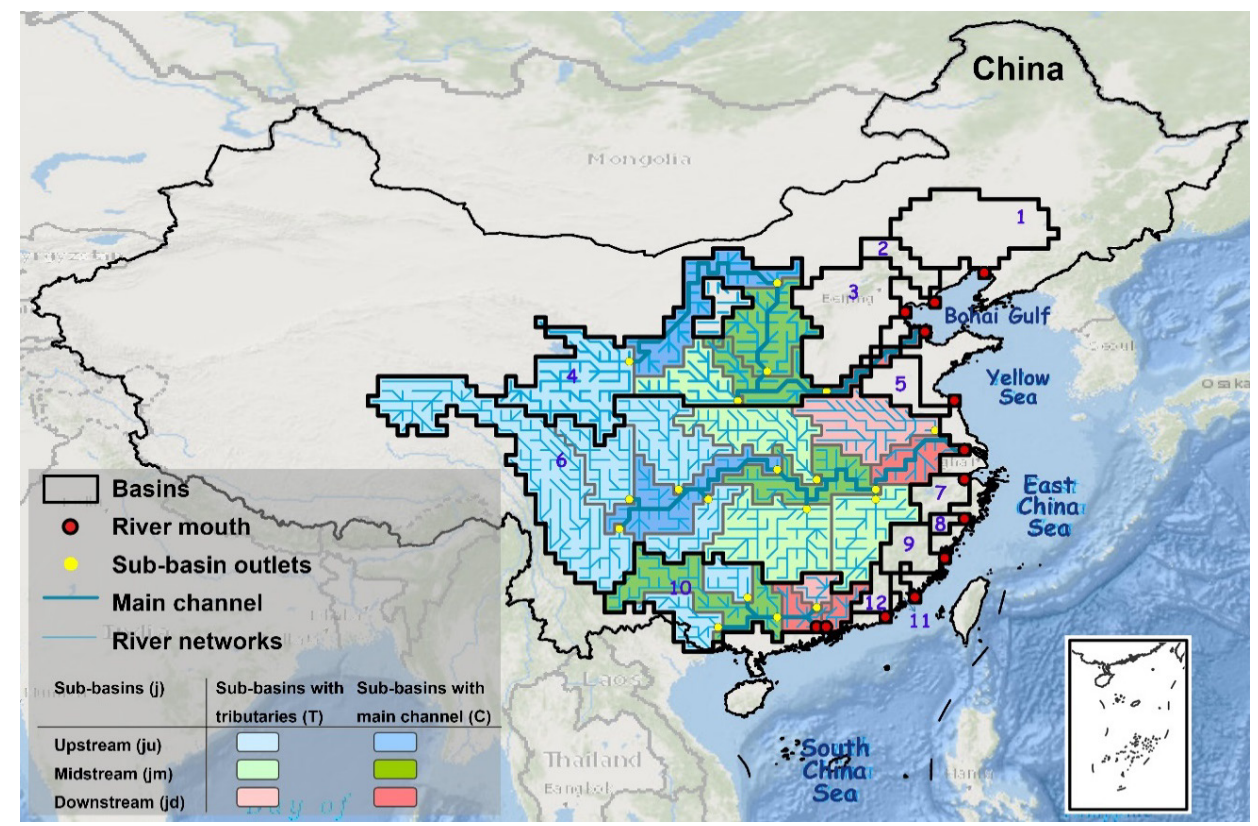

Figure 5.1 The 12 Chinese rivers draining into the Bohai Gulf, Yellow Sea, East China Sea and South China Sea. The names of the rivers are: the Liao (1 in the Figure), Luan (2), Hai (3) and Yellow (4) rivers draining into the Bohai Gulf; the Huai River (5) draining into the Yellow Sea; the Yangtze (6), Fuchun (7), Oujiang (8) and Menjiang (9) rivers draining into the East China Sea; the Pearl (10), Jiulong (11) and Hanjiang (12) rivers draining into the South China Sea. River drainage area is based on the global drainage direction map on 30 arc minute resolution (DDM-30) (Döll and Lehner, 2002). The country boundary for China is from (RESDC, 2013).

\subsubsection{MARINA 2.0}

We developed the MARINA 2.0 model to quantify river export of total dissolved N (TDN) and P (TDP) by 12 Chinese rivers, by source, and at the sub-basins scale for 2012 and 2050. TDN is the sum of dissolved inorganic (DIN) and dissolved organic (DON) N. TDP is the sum of dissolved inorganic (DIP) and dissolved organic (DOP) P. MARINA 2.0 quantifies dissolved $\mathrm{N}$ and $\mathrm{P}$ export by rivers as a function of $\mathrm{N}$ and $\mathrm{P}$ inputs to surface waters (rivers) from diffuse and point sources and retentions of $\mathrm{N}$ and $\mathrm{P}$ in rivers based on the overall equation below:

$M_{F . y . j}=\left(R_{\text {Sdiff.y.j }}+\right.$ RSpntF.y.j $)$ FEriv.F.outlet.j $\cdot$ FEriv.F.mouth.j

Where $\mathrm{M}_{\mathrm{F} . \mathrm{y} . \mathrm{j}}$ is river export of $\mathrm{N}$ and P in form F (DIN, DON, DIP, DOP) by source y from sub-basin $\mathrm{j}^{\left(\mathrm{kg}_{\text {year }}{ }^{-1}\right) \text {. RSdif }}$.y.j is $\mathrm{N}$ and $\mathrm{P}$ inputs in form $\mathrm{F}$ to rivers (surface waters) from 
diffuse sources y in sub-basin $\mathrm{j}\left(\mathrm{kg}_{\text {year }}{ }^{-1}\right)$. RSpntF.y.j is $\mathrm{N}$ and $\mathrm{P}$ inputs in form $\mathrm{F}$ to rivers from point sources y in sub-basin $\mathrm{j}\left(\mathrm{kg}_{\text {year-1). }}{ }^{-}\right.$. FE riv.F.outlet.j is the fraction of $\mathrm{N}$ and $\mathrm{P}$ in form F exported to the outlet of sub-basin $\mathrm{j}(0-1)$. FEriv.F.mouth.j is the fraction of $\mathrm{N}$ and $\mathrm{P}$ in form $\mathrm{F}$ exported from the outlet of sub-basin $\mathrm{j}$ to the river mouth $(0-1)$. We summarized the

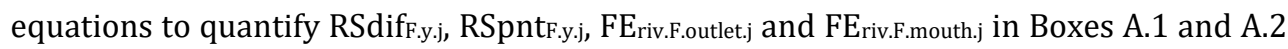
in Appendix IV.

The MARINA 2.0 model is based on the MARINA 1.0 model, developed by Strokal et al. (2016a) for six large rivers: Yellow, Yangtze, Pearl, Liao, Huai and Hai rivers in China. MARINA 1.0 quantifies river export of four nutrient forms (DIN, DON, DIP and DOP) to the river mouth by sub-basins from different sources. In this study, the MARINA 1.0 model was modified and updated to MARINA 2.0 for 12 rivers in China. We focused on rivers that contain at least five grids of $0.5^{\circ} \times 0.5^{\circ}$ spatial resolution.

MARINA 2.0 differs from MARINA 1.0 in the following aspects. First, we included in MARINA 2.0 human waste from rural population that is connected to sewage systems. This source was not considered in MARINA 1.0 because the rural population in China had very limited connections to sewage systems in 2000 (MOHURD, 2001). Second, we created the basin delineation for the Chinese rivers using the 30 -arcminute $\left(0.5^{\circ} \times 0.5^{\circ}\right)$ spatial resolution Drainage Direction Map (DDM-30) (Döll and Lehner, 2002). The MARINA 1.0 model (Strokal et al., 2016) used the 30-arcminute Simulated Topological Networks (STN-30) (Vörösmarty et al., 2000). Third, we updated the approach to quantify human waste based on the MARINA-Global model (Strokal et al., 2019). This was done by adjusting the method to calculate protein N intake using units of 2005 US\$ instead of 1995 US\$ for GDP (gross domestic product) (see equations in Box A.1 in Appendix IV).

To apply the MARINA 2.0 model to Chinese rivers for 2012, we updated the model inputs for 1) point sources (e.g., GDP, population, sewage connections, sewage treatment) mainly based on Chinese statistics, 2) diffuse sources (e.g., use of synthetic fertilizers, animal manure) based on the NUFER (NUtrient flows in Food chains, Environment and Resources use) model at the county scale (Wang et al., 2018b), and 3) hydrology (e.g., river discharge) with simulations based on the VIC (Variable Infiltration Capacity) model from the study of (Van Vliet et al., 2016a; Van Vliet et al., 2016b). The overview of the updated model inputs, their sources, and the methods that we used to aggregate these model inputs to the sub-basin scale are in Figure A.1, Tables A.2 and A.3 in Appendix IV. 
We evaluated the performance of MARINA 2.0 by comparing the modeled results with measurements at the river mouths (Supplementary Information D in Appendix IV), modeled results from other studies, and by performing sensitivity analysis. The results show that MARINA 2.0 has an acceptable performance in quantifying river export of nutrients for China. Detailed discussions on our model evaluation are available in Section 5.4.1.

Indicator for Coastal Eutrophication. Based on the modeled results by MARINA 2.0, we assessed the potential impacts of river export of TDN and TDP on coastal eutrophication in 2012 and 2050 for the SSP-RCP scenarios. To do this, the Indicator for Coastal Eutrophication Potential (ICEP) developed by Billen and Garnier (2007) was used. The ICEP indicator expresses the potential for the new production of non-siliceous (possibly harmful) algae in coastal waters when receiving excess $\mathrm{N}$ or $\mathrm{P}$ over silica from rivers. ICEP is calculated by comparing the N, P and Si loads to the Redfield molar ratios (C:N:P: Si ratios 106:16:1:20) (see the equations below to quantify ICEP). Either N- or P- ICEPs were calculated depending on if $\mathrm{N}$ or $\mathrm{P}$ is limiting (i.e. $\mathrm{N}$ is limiting if $\mathrm{N}: \mathrm{P}<16$; otherwise $\mathrm{P}$ is limiting). Positive ICEP value indicates an excess of $\mathrm{N}$ or $\mathrm{P}$ over $\mathrm{Si}$, which leads to high potentials for blooms of harmful algae. Negative ICEP value indicates a low potential for blooms of harmful algae. Following the approach of (Garnier et al., 2010), we calculated ICEPs for the 12 Chinese rivers following the equations:

$$
\begin{aligned}
& \mathrm{N}-\mathrm{ICEP}=[\mathrm{TDNflx} /(14 \cdot 16)-\mathrm{DSifl} /(28 \cdot 20)] \cdot 106 \cdot 12 \text { if } \mathrm{N}: \mathrm{P}<16(\mathrm{~N} \text { is limiting }) \\
& \mathrm{P}-\mathrm{ICEP}=[\mathrm{TDPfl} / 31-\mathrm{DSifl} /(28 \cdot 20)] \cdot 106 \cdot 12 \text { if } \mathrm{N}: \mathrm{P}>16(\mathrm{P} \text { is limiting })
\end{aligned}
$$

Where TDNflx, TDNflx are the fluxes of total dissolved nitrogen and phosphorus, respectively ( $\mathrm{kg} \mathrm{km}^{-2}$ year-1). DSiflx is the flux of total dissolved silica $\left(\mathrm{kg} \mathrm{km}^{-2}\right.$ year-1). TDNflx and TDPflx were based on our quantified river export of TDN and TDP by the MARINA 2.0 model in this study. DSiflx were from the Global NEWS-2 (Global Nutrient Export from WaterSheds) model (Seitzinger et al., 2010) (see Table A.5 in Appendix IV for values of DSi). 
The Yellow River: 6 sub-basins

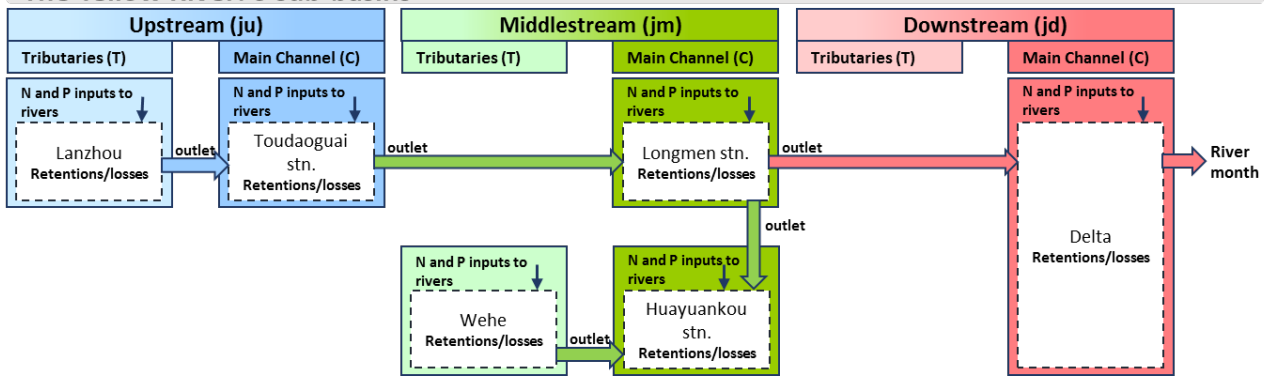

The Yangtze River: 11 sub-basins
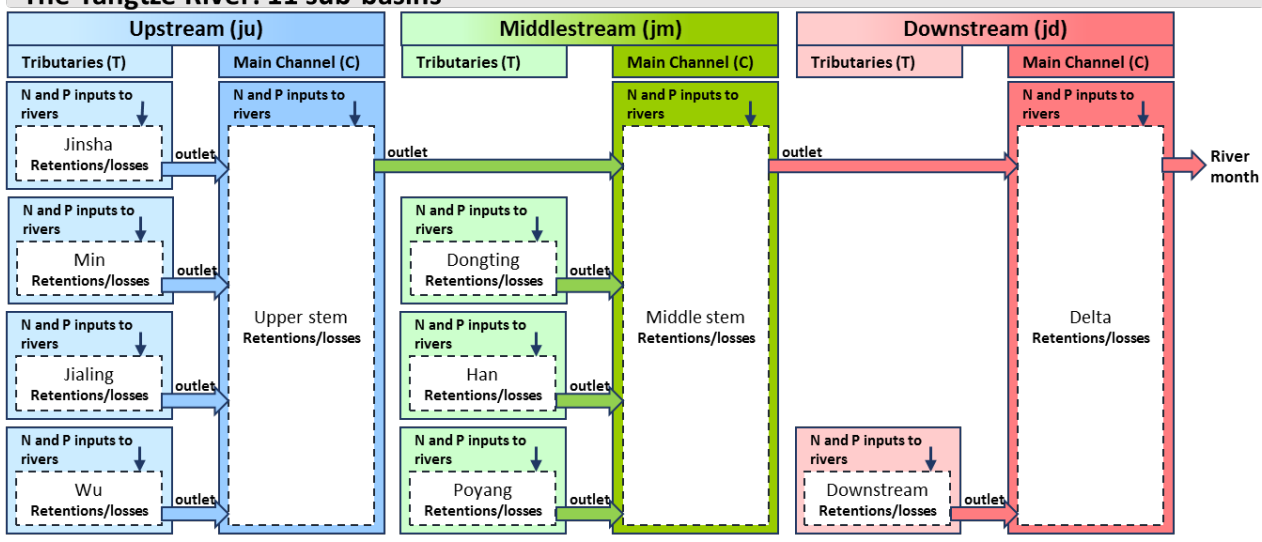

The Pearl River: 6 sub-basins
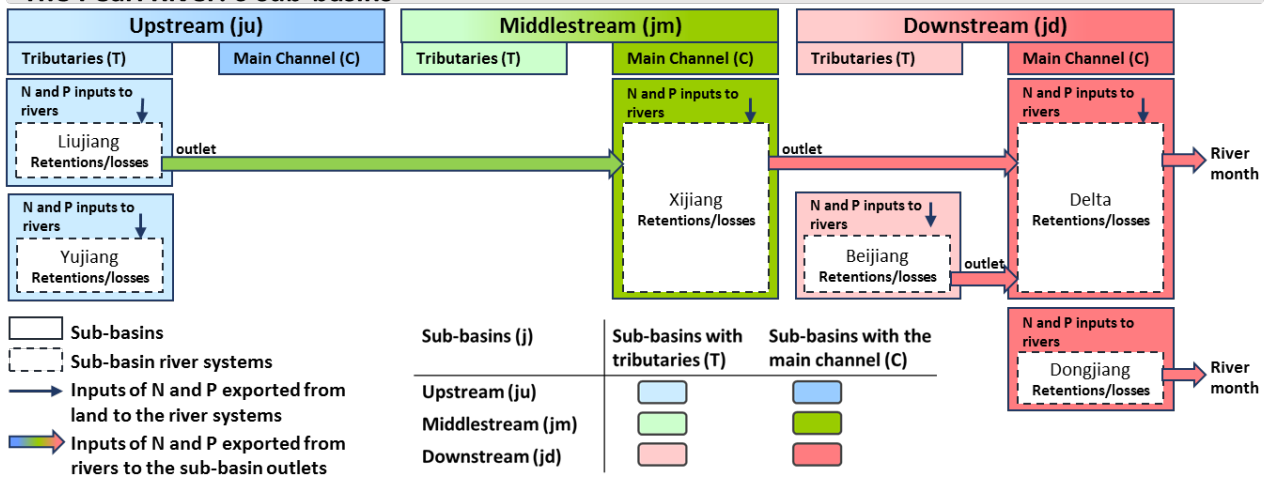

Figure 5.2 The sub-basin scale modeling framework for the Yellow, Yangtze and Pearl rivers in the MARINA 2.0 model (Model to Assess River Inputs of Nutrients to seAs). This modeling framework is developed based on the MARINA 1.0 model (Strokal et al., 2016a). See Figure 5.1, and Figures A.3-A.5 in Appendix IV for the locations of the rivers and their sub-basins. 


\subsubsection{Scenarios}

We quantified river export of TDN and TDP by rivers for 2050. Five Shared Socioeconomic Pathways (SSPs) were selected for strong, rapid (SSP1 - "Sustainability", SSP5"Fossil-fueled"), moderate (SSP2 - "Middle of the Road"), and slow (SSP3 - "Regional Rivalry", SSP4 - "Inequality") socio-economic developments (O'Neill et al., 2014). SSP1 assumes a gradual shift towards sustainability with low population growth, economic growth, efficient use of resources, high technic solutions and improved environmental policies for environmental pollution. SSP2 assumes moderate progress towards sustainability, moderate population growth, some improvements in resource use efficiencies, environment policies only for local pollution. SSP3 assumes a fragmented world to achieve energy and food security goals with high challenges including strong environmental degradation, high population growth, limited improvements in techniques and environmental policies for pollution. SSP4 assumes inequalities in population growth, urbanization, economy, and environmental policies among countries and regions in the countries. SSP5 assumes integrated global societies with low population growth, high consumption of fossil fuels, and highly management environmental conditions at local scales (O’Neill et al., 2017). Two Representative Concentration Pathways (RCPs) were selected for the lowest (RCP2.6) and highest (RCP8.5) greenhouse gas (GHGs) concentrations for climate change (Van Vuuren et al., 2011).

The six scenarios combining SSPs and RCPs are: SSP1-RCP2.6, SSP2-RCP2.6, SSP2-RCP8.5, SSP3-RCP8.5, SSP4-RCP2.6, SSP5-RCP8.5. These combinations were selected based on the SSP-RCP matrix from Kok (2016), and added SSP2-RCP8.5 to analyze the impact of climate change on river export of nutrients.

Model inputs for MARINA 2.0 for hydrology (e.g., river discharge) for RCP 2.6 and RCP8.5 were from Van Vliet et al. (2016a). Van Vliet et al. (2016a) implemented bias-corrected model output of five global climate models from ISI-MIP (Inter-Sectoral Impact Model Intercomparison Project) (Hempel et al., 2013) for both RCP2.6 and RCP8.5 in the VIC hydrological model (Figure A.1 and Table A.3 in Appendix IV). Most model inputs for agricultural activities were derived by implementing the SSP storylines for China's food system from Wang et al. (2017) to the NUFER model at the county scale (Figure A.1 and Table A.3 in Appendix IV). Model inputs for calculating nutrient export from human waste for the selected scenarios were estimated based on our assumptions on 1) the fraction of 
population connected to sewage systems; and 2) $\mathrm{N}$ and $\mathrm{P}$ removal efficiencies during sewage treatment based on the SSP-RCP storylines and existing studies (O'Neill et al., 2017; Van Drecht et al., 2009) (Table A.4 in Appendix IV). Important model inputs are presented in Figures B.1-B.8 in Appendix IV.

(A)

SSP1

SSP2

SSP3

SSP4

SSP5

(Sustainability) (Middle of the

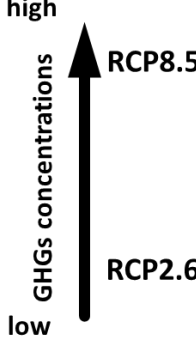

Road)

(Regional

(Inequality)

(Fossil-fuelled

Rivalry)

Development)

(B)

\begin{tabular}{|c|c|c|c|c|c|c|c|c|}
\hline \multirow[t]{2}{*}{ Scenarios } & \multicolumn{5}{|l|}{ Agriculture } & \multicolumn{2}{|c|}{ Sewage system } & \multirow{2}{*}{$\begin{array}{l}\text { Climate } \\
\text { mitigation }\end{array}$} \\
\hline & Productivity & $\begin{array}{l}\text { Agricultural } \\
\text { Land }\end{array}$ & $\begin{array}{l}\text { Food and } \\
\text { feed } \\
\text { imports }\end{array}$ & $\begin{array}{l}\text { Recycling of } \\
\text { nutrients } \\
\text { (e.g., crop } \\
\text { residues, } \\
\text { animal } \\
\text { manure) }\end{array}$ & $\begin{array}{l}\text { Mitigation } \\
\text { of nutrient } \\
\text { losses to } \\
\text { the air }\end{array}$ & $\begin{array}{l}\text { Sewage } \\
\text { connection }\end{array}$ & $\begin{array}{l}\text { Sewage } \\
\text { treatment }\end{array}$ & \\
\hline SSP1-RCP2.6 & +++ & - & * & +++ & +++ & +++ & +++ & +++ \\
\hline SSP2-RCP2.6 & ++ & $\cdots$ & + & ++ & ++ & ++ & ++ & +++ \\
\hline SSP2-RCP8.5 & ++ & $\cdots$ & + & ++ & ++ & ++ & ++ & - \\
\hline SSP3-RCP8.5 & - & - & - & - & - & $*$ & $*$ & - \\
\hline SSP4-RCP2.6 & + & - & ++ & + & + & + & + & +++ \\
\hline SSP5-RCP8.5 & + & -- & +++ & +++ & ++ & ++ & ++ & - \\
\hline
\end{tabular}

Note: + + + strong increase, + + moderate increase, + slight increase, - - - strong decrease, - - moderate decrease, - slight decrease, * no changes

Figure 5.3 (A) The six scenarios combining Shared Socio-economic Pathways (SSPs) and Representative Concentration Pathways (RCPs), presented in a SSP-RCP matrix, (B) the qualitative storylines for agriculture, sewage systems and climate mitigation in China in the SSPRCP scenarios. SSP2-RCP8.5 is not the most likely combination, but was added to analyze the impact of climate change on river export of nutrients. The storylines for agriculture are based on Wang et al. (2017). The quantitative scenarios for nutrient flows in agriculture and sewage systems, and for hydrology (e.g., river discharge) are available in Figures B.1-B.8 in Appendix IV. 


\subsubsection{Hotspots for $N$ and $P$ losses}

In order to better understand how socio-economic change influences future river export of nutrients, we analyzed hotpots for $\mathrm{N}$ and $\mathrm{P}$ losses to rivers from diffuse (agriculture) and point (agriculture and human waste) sources for the SSPs. "Hotspots" are the regions where $\mathrm{N}$ and $\mathrm{P}$ losses to rivers are higher than in other regions, as a result of nutrient management in socio-economic activities. We followed the approach of Wang et al. (2018b) to identify hotspots for $\mathrm{N}$ and $\mathrm{P}$ losses from agriculture. Our hotspots are presented at the gridded $\left(0.5^{\circ} \times 0.5^{\circ}\right)$ scale. Below we describe our approach to identify the hotspots for diffuse and point sources.

Diffuse sources include leaching, runoff and erosion of nutrients (e.g., synthetic fertilizers, animal manure) in agriculture. To identify the hotspots, we first took the NUFER results for $\mathrm{N}$ and $\mathrm{P}$ losses to waters (rivers and groundwater) from leaching, runoff and erosion in agriculture at the county scale. These results were aggregated from counties to grids $\left(0.5^{\circ} \times 0.5^{\circ}\right)$ as described in Table A. 3 and Figure A. 2 in Appendix IV. Next, the N and P losses were averaged by the area of the grids and were grouped into four groups (Figure 5.9 and Figure C. 4 in Appendix IV). The ranges of $\mathrm{N}$ and P losses for the four groups were the same as defined by Wang et al. (2018b), who identified hotspots for N and P losses from food production to the environment. The top group (ranking by $\mathrm{N}$ and P losses) were considered as hotspots. Based on Wang et al. (2018b), for N, grids with losses to waters from diffuse sources exceeding $2452 \mathrm{~kg} \mathrm{~N} \mathrm{~km}^{-2}$ year-1 were qualified as hotspots. For $\mathrm{P}$, grids with losses from diffuse sources exceeding $147 \mathrm{~kg} \mathrm{P} \mathrm{km}^{-2}$ year-1 were considered as hotspots.

Point sources include direct discharge of manure, discharge of collected (through sewage systems) and uncollected (not through sewage systems) human waste. The same approach to identify hotspots as for diffuse sources was applied. The $\mathrm{N}$ and P losses to rivers from discharge of collected and uncollected human waste were not available from NUFER. Thus we quantified these on the gridded scale using the MARINA approach (see Box A.1) based on the gridded population and their connection to sewage systems (Figure A.1 in Appendix IV). Wang et al. (2018b) did not identify the hotspots for $\mathrm{N}$ and P losses to rivers from discharge of collected and uncollected human waste. We, therefore, identified the hotspots for these sources based on the number of hotspots (grids) for the diffuse source. For diffuse sources, we identified around 575 grids as hotpots (i.e. 14\% 
grid cells with highest $\mathrm{N}$ and $\mathrm{P}$ losses). The range of $\mathrm{N}$ and $\mathrm{P}$ losses for these top 575 grids (ranking by $\mathrm{N}$ or P losses) was thus used as the range for hotpots for discharge of collected and uncollected human waste (Figure 5.9, and Figure C.4 in Appendix IV).

\subsection{Results}

\subsubsection{River export of nutrients in $\mathbf{2 0 1 2}$}

In 2012 the 12 Chinese rivers exported in total 3287 kton of DIN, 1567 kton of DON, 295 kton of DIP, and 411 kton of DOP to seas (Figure 5.4). Around half of these nutrients were exported to the East China Sea. The remainder was exported to the Bohai Gulf $111-23 \%$ depending on the nutrient form), Yellow Sea (5-13\%) and South China Sea (14-32\%). The Hai and Liao rivers, Yangtze River, and Pearl River were the dominant exporters of nutrients to the Bohai Gulf, East China Sea and South China Sea, respectively. Below we describe the nutrient export by rivers to each of the four seas and their sources at the subbasin scale in 2012 .

The Bohai Gulf received 370 kton of DIN, 309 kton of DON, 62kton of DIP, and 94 kton of DOP in 2012 (Figure 5.4). About 68-80\% of these nutrients originated from human activities (e.g., use of synthetic fertilizers, direct discharge of manure and uncollected human waste) in the Hai and Liao rivers (Figures 5.5 and 5.6, Figures C.1 and C.2 in Appendix IV). Direct discharge of animal manure to rivers was responsible for $41 \%$ of DIN, and for 68-83\% of DON, DIP and DOP exports to the Bohai Gulf. The Yellow River has a large drainage area of $758,715 \mathrm{~km}^{2}$ but only contributed by less than $17 \%$ to the TDN and TDP export to the Bohai Gulf in 2012. This is largely caused by the relatively high retentions of nutrients in the Yellow River basin (Figure C.6 in Appendix IV).

The Huai River is the only river flowing into the Yellow Sea in our model. In 2012, the Huai River exported 174 kton of DIN, 77 kton of DON, 37 kton of DIP and 26 kton of DOP to the Yellow Sea (Figure 5.4). Direct discharge of animal manure was the most important source for DON, DIP and DOP, and accounted for 63-86\% of these forms entering the Yellow Sea (Figures 5.5 and 5.6, Figures C.1 and C.2 in Appendix IV). Use of synthetic fertilizers was another important source and accounted for $42 \%$ of the DIN inputs to the Yellow Sea.

The East China Sea received 1699 kton of DIN, 904 kton of DON, 140 kton of DIP and 233 kton of DOP in 2012 (Figure 5.4). More than $90 \%$ of these nutrients were exported by the Yangtze River. Close to $85 \%$ of DIN and DIP, and 66-69\% of DON and DOP exported by the 
Yangtze River to coastal waters were from activities in middle-stream and downstream sub-basins (Figures 5.5 and 5.6, Figures C.1, C.2 and C.5 in Appendix IV). The main sources of nutrient export to the East China Sea were direct discharge of animal manure, use of synthetic fertilizers, and discharge of uncollected human waste (Figures 5.5 and 5.6, Figures C.1 and C.2 in Appendix IV). In 2012, these sources accounted for 53-94\% of the nutrient inputs to seas depending on the nutrient forms. Direct discharge of animal manure was the most important source for DON, DIP and DOP. Other sources such as use of synthetic fertilizers are important for DIN inputs to the East China Sea.

The South China Sea received 1044 kton of DIN, 275 kton of DON, 55 kton of DIP and 59 kton of DOP in 2012 (Figure 5.4). About 72-84\% of these nutrients were exported by the Pearl River. Most of the nutrients (79-86\%) exported by the Pearl River to coastal waters originated from the activities in middle-stream and downstream sub-basins (Figures 5.5 and 5.6, Figures C.1, C.2 and C.5 in Appendix IV). Direct discharge of animal manure, and direct discharge of uncollected human waste were responsible for $57 \%$ of DON, $64 \%$ of DIP, and 88\% of DOP entering the South China Sea in 2012 (Figures 5.5 and 5.6, Figures C.1 and C.2 in Appendix IV). Atmospheric N deposition, biological N2 fixation and use of synthetic fertilizers were the main sources of DIN export to the South China Sea.

Based on the river export of TDN and TDP, we assessed the potential impacts on coastal eutrophication for the 12 rivers. In 2012, the ICEP values range from -39 to +51 C-eq. km${ }^{2}$ year $^{-1}$ among rivers (Figure 5.8). Almost all 12 rivers have positive ICEP values $(>0)$, indicating high potentials for harmful algae to develop in coastal waters. The Oujiang and Fuchun rivers are exceptions with negative ICEP values $(<0)$, indicating low potentials to cause coastal eutrophication. 


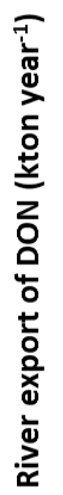

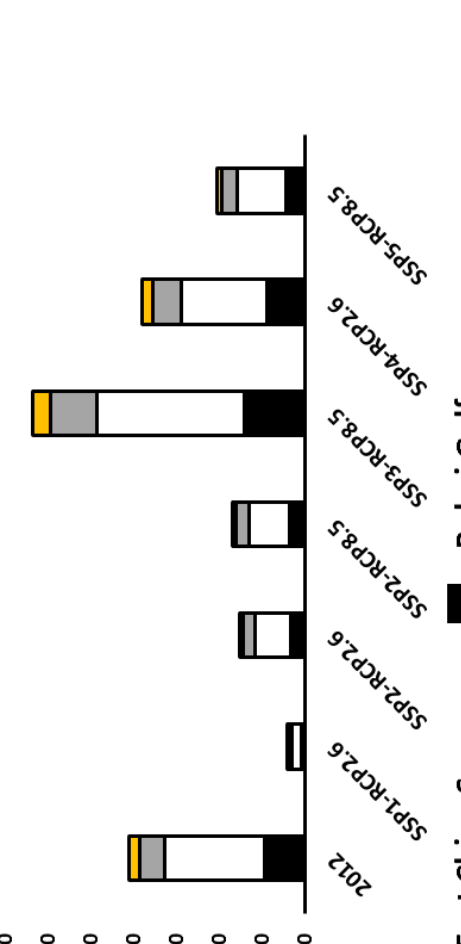

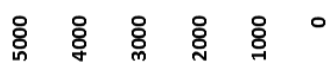

용 \& \& 8 융 \&

હే ๘ั

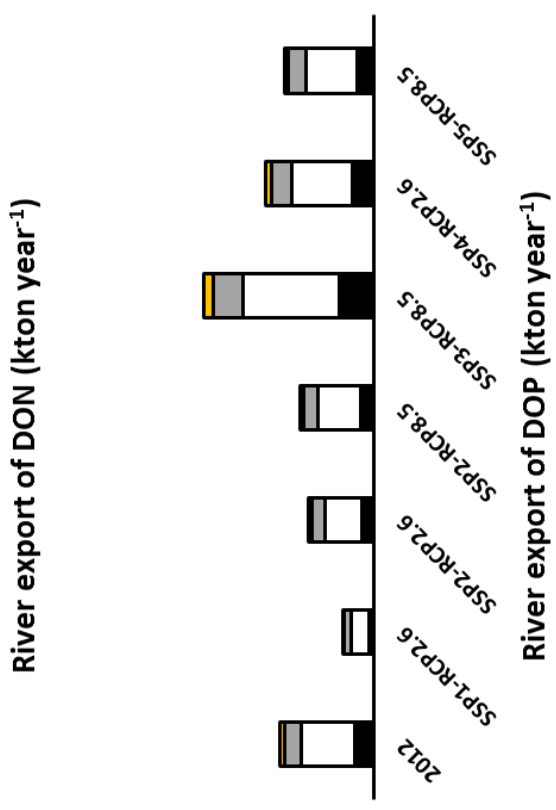

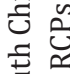

苛

कृ

का

च

告 焉

品

ఏ్d

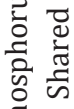

릉

ซี

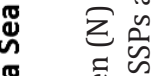

๕

坣芯.

(ิ)

递

Ż :

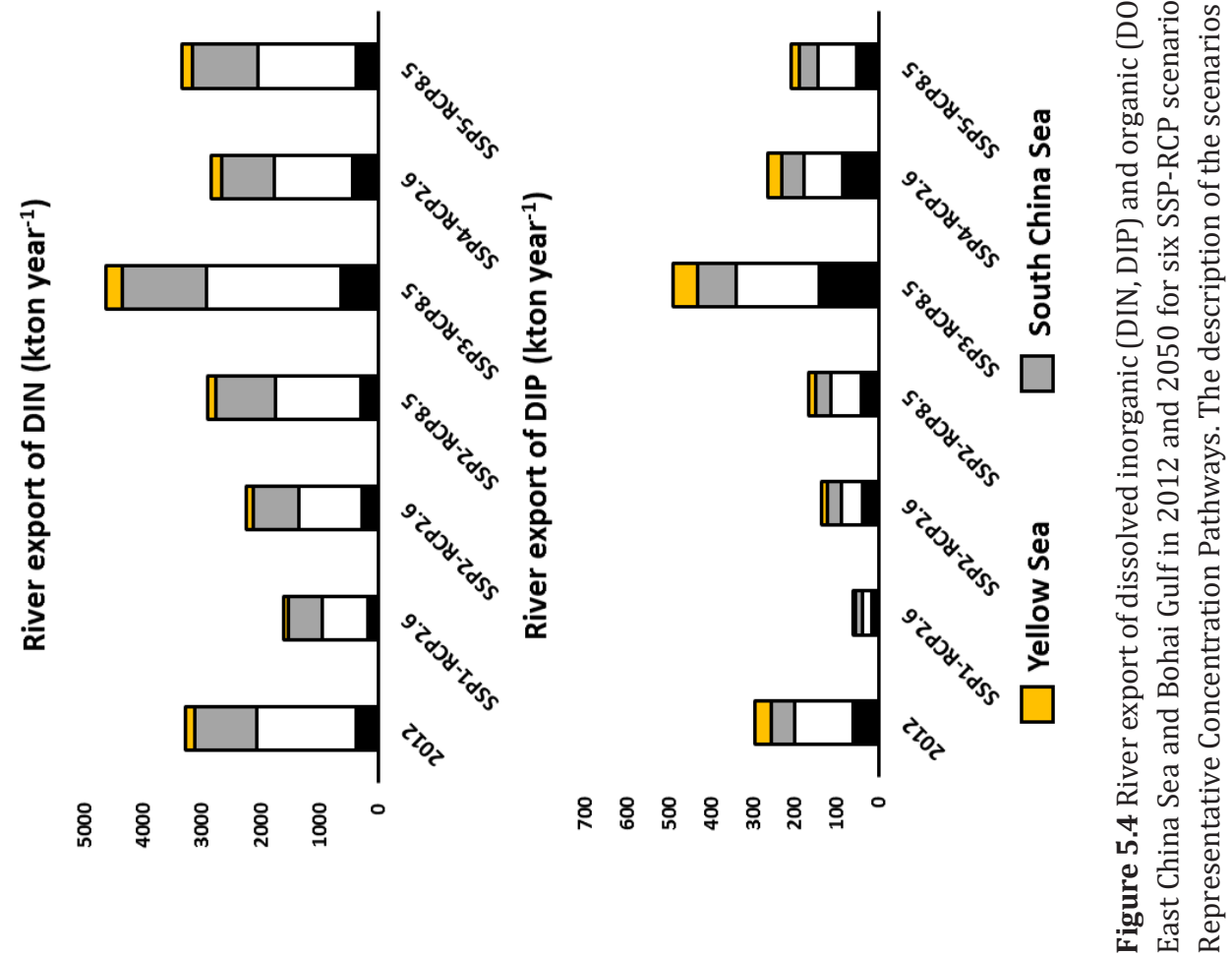



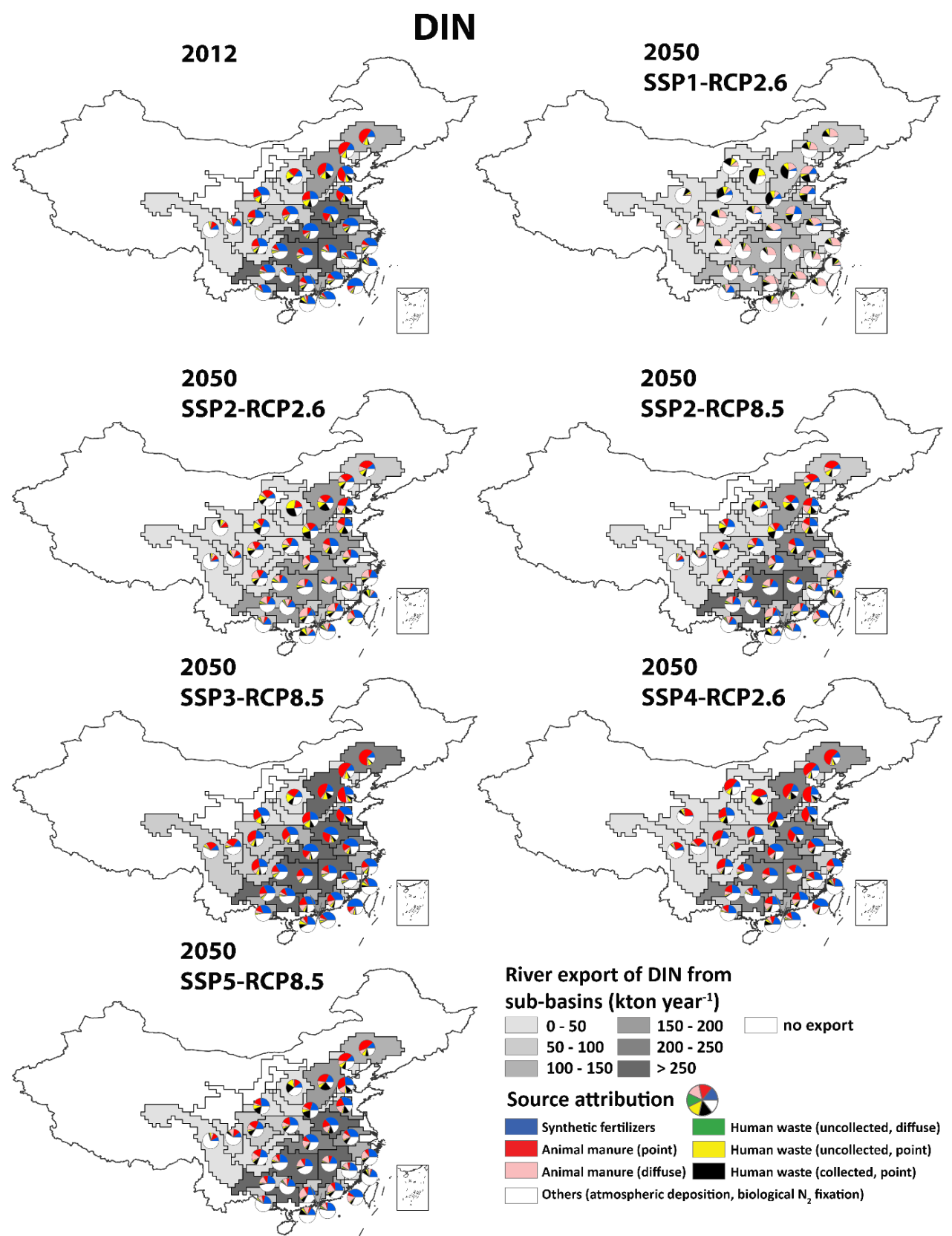

River export of DIN from sub-basins (kton year-1)
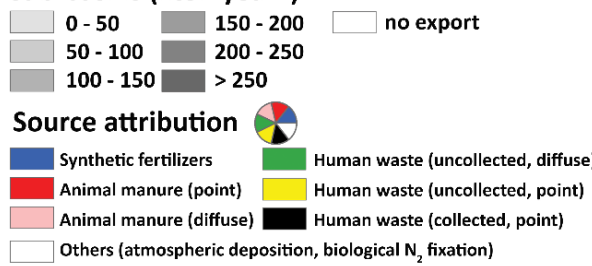

Figure 5.5 River export of dissolved inorganic nitrogen (DIN, kton year-1) from sub-basins of 12 Chinese rivers by source in 2012 and 2050 for six SSP-RCP scenarios. SSPs are the Shared Socioeconomic Pathways. RCPs are the Representative Concentration Pathways. Details on the SSP-RCP scenarios are in Section 5.2.3. The names and locations of the rivers are in Figure 5.1, and Figures A.3-A.5 in Appendix IV. 


\section{DIP}
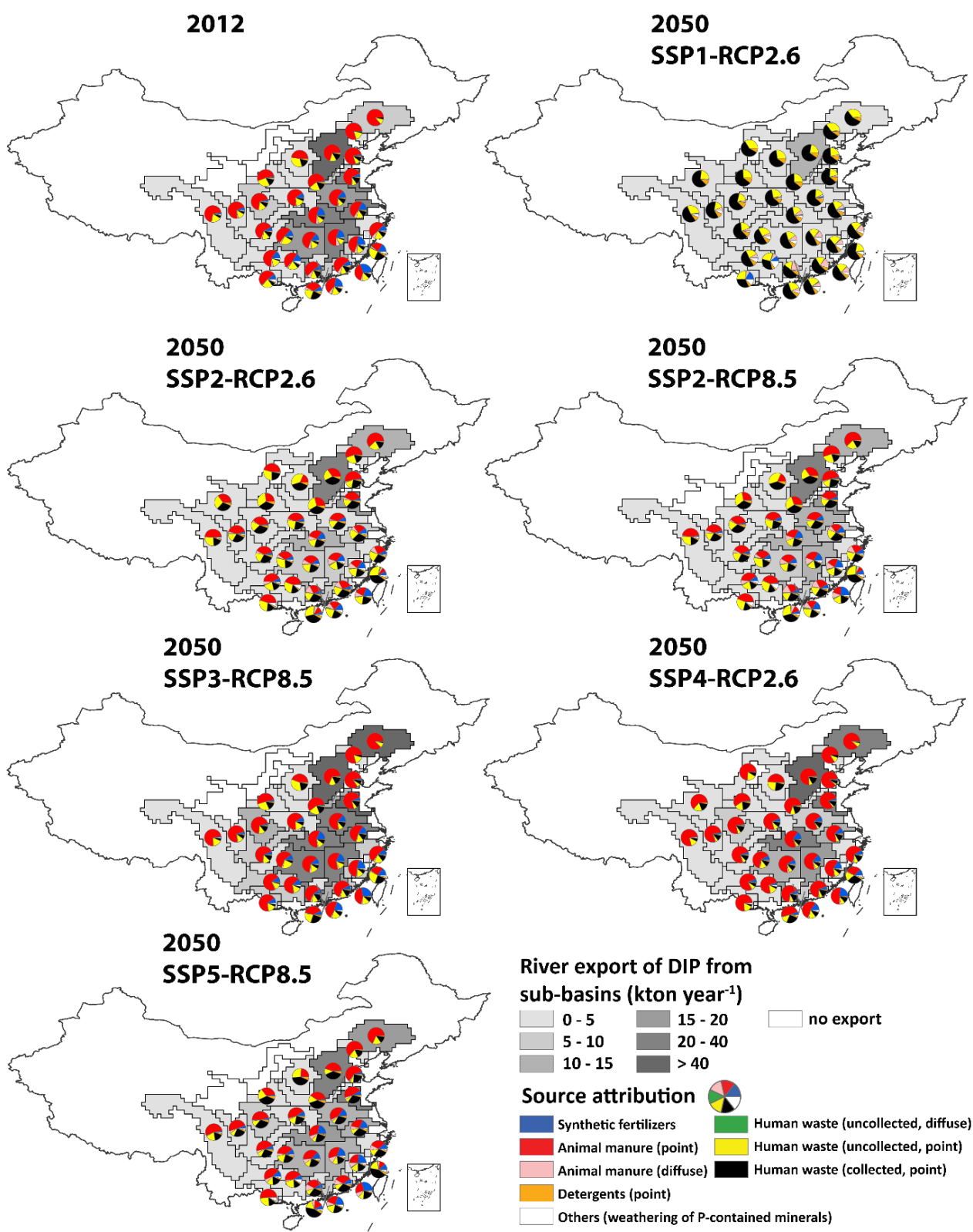

River export of DIP from

sub-basins (kton year ${ }^{-1}$ )

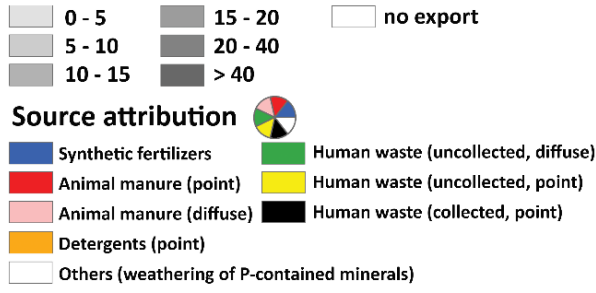

Figure 5.6 River export of dissolved inorganic phosphorus (DIP, kton year-1) from sub-basins of 12 Chinese rivers by source in 2012 and 2050 for six SSP-RCP scenarios. SSPs are the Shared Socio-economic Pathways. RCPs are the Representative Concentration Pathways. Details on the SSP-RCP scenarios are in Section 5.2.3. The names and locations of the rivers are in Figure 5.1, and Figures A.3-A.5 in Appendix IV. 


\subsubsection{Future river export of nutrients}

In 2050 rivers and coastal waters may be much more polluted or much cleaner than today, depending on the SSP-RCP scenarios. Below we describe the changes in river export of nutrients for each of the six scenarios in detail.

In SSP1-RCP2.6, total river export of dissolved N and P by Chinese rivers in 2050 is projected to be $50-90 \%$ lower than in 2012 depending on the nutrient forms (Figure 5.4). The decreases also vary among sub-basins. In most sub-basins the river export of $\mathrm{N}$ or $\mathrm{P}$ is reduced by up to half of the amount in 2012 (Figure 5.7 and C.3). The large decreases in nutrients export are explained by the assumed restricted discharge of animal manure, reduced use of synthetic fertilizers, and improved sewage connections and wastewater treatment in this scenario. As a result, atmosphere $\mathrm{N}$ deposition and biological $\mathrm{N} 2$ fixation become the dominant sources of DIN in river export, and are responsible for up to $87 \%$ of DIN exported from sub-basins. Discharge of collected (through sewage systems) and uncollected human waste are the most important sources of DON, DIP, and DOP in this scenario (Figures 5.5 and 5.6, Figures C.1 and C.2 in Appendix IV).

SSP2-RCP2.6 and SSP2-RCP8.5 assume the same moderate socio-economic developments, but with different level (low for RCP2.6, high for RCP8.5) of greenhouse gas concentrations and related changes in climate in 2050. River export to all four seas is projected to decrease between 2012 and 2050 for both scenarios. The four $\mathrm{N}$ and $\mathrm{P}$ forms entering Chinese Seas are 30-58\% lower in SSP2-RCP2.6 than in 2012, and are 11-54\% lower in SSP2-RCP8.5 (Figure 5.4). Direct discharge of animal manure is projected to reduce, but may still remain the main source of DON, DIP, and DOP. For DIN, other sources such as atmospheric $\mathrm{N}$ deposition and biological $\mathrm{N} 2$ fixation will become the dominant sources for most sub-basins (Figures 5.5 and 5.6, Figures C. 1 and C.2 in Appendix IV).

River export of dissolved N and P is projected to increase considerably between 2012 and 2050 in SSP3-RCP8.5. We calculate increases in river export of DIN by $41 \%$, of DIP by $67 \%$ and of DOP by 54\% (Figure 5.4). The amount of DON may almost double between 2012 and 2050. The large increase in nutrients transported to coastal waters is related to the intensive human activities in agriculture and urbanization, and the poor nutrient management (e.g., overuse of synthetic fertilizers, insufficient wastewater treatment) in SSP3-RCP8.5. Direct discharge of manure is expected to remain the dominant source of DON, DIP, and DOP. Use of synthetic fertilizers is expected to be an important source, and 
contributes by up to $50 \%$ to river export of DIN among the sub-basins (Figures 5.5 and 5.6, Figures C.1 and C.2 in Appendix IV).

In SSP4-RCP2.6 and SSP5-RCP8.5 scenarios, river export of dissolved N and P in 2050 is slightly lower or slightly higher than in 2012 depending on the nutrient forms (Figure 5.4). Changes in nutrients export vary largely among sub-basins and nutrient forms as a net effect of future changes in human activities, nutrient management, and river discharges. For example, most sub-basins of the Pearl River are expected to deliver increased amounts of DON, but decreased amounts of DIN to seas in SSP4-RCP2.6 in 2050. This is due to the reduced use of fertilizers for crops, and the reduced direct discharge of manure in this scenario. In these two scenarios, direct discharge of manure is expected to remain the dominant source of DON, DIP, and DOP. Use of synthetic fertilizers remains an important source for river export of DIN (Figures 5.5 and 5.6, Figures C.1 and C.2 in Appendix IV).

As a result of the abovementioned trends, the potential of the river export of nutrients to cause coastal eutrophication may be higher or lower in the future depending on the SSPRCP scenarios (Figure 5.8). In 2012, the ICEP values range from -39 to +51 C-eq. $\mathrm{km}^{-2}$ year 1 among rivers. For the scenario focusing on sustainability (SSP1-RCP2.6) the ICEP values are with a range of -60 to $+8 \mathrm{C}$-eq. $\mathrm{km}^{-2}$ year-1 among rivers, which are much lower than that in 2012. Several rivers (e.g., rivers draining into the South China Sea and East China Sea, and the Luan River) have negative ICEP values, indicating low potentials for harmful algae to develop. In contrast, the potentials for coastal eutrophication are much higher for all rivers in SSP3-RCP8.5 than in 2012. The ICEP values of rivers range from -30 to +94 Ceq. $\mathrm{km}^{-2}$ year-1. Positive ICEP values are calculated for all rivers except for the Oujiang River. The ICEP values are lower in 2050 than in 2012 in both SSP2-RCP2.6 and SSP2RCP8.5. They range from -49 to +32 C-eq. $\mathrm{km}^{-2}$ year-1 $^{-1}$ in SSP2-RCP2.6, and from -47 to +37 C-eq. $\mathrm{km}^{-2}$ year-1 SSP2-RCP8.5 among rivers. However, the potentials of most rivers except for Oujiang and Fuchun to cause coastal eutrophication remain high in these two scenarios indicated by the positive ICEP values. ICEP values are slightly lower in SSP5RCP8.5 than in 2012, ranging from -45 to +45 C-eq. $\mathrm{km}^{-2}$ year-1. In SSP4-RCP2.6, the potentials for coastal eutrophication are higher or lower than in 2012 depending on the rivers. For example, ICEP is calculated to increase at the river mouth of Hai. We quantified increased river export of TDN, and decreased river export of TDP by Hai in SSP4-RCP2.6 
(Figure 5.7). According to the N:P ratio, $\mathrm{N}$ is limiting for this river (Figure C.1 in Appendix IV). Thus a higher ICEP is calculated for Hai taking into account changes in DSi export (see equations 2 and 3 in Section 5.2.2). Positive ICEP values are calculated for all rivers except for the Oujiang and Fuchun rivers in these SSP4-RCP2.6 and SSP5-RCP8.5. 

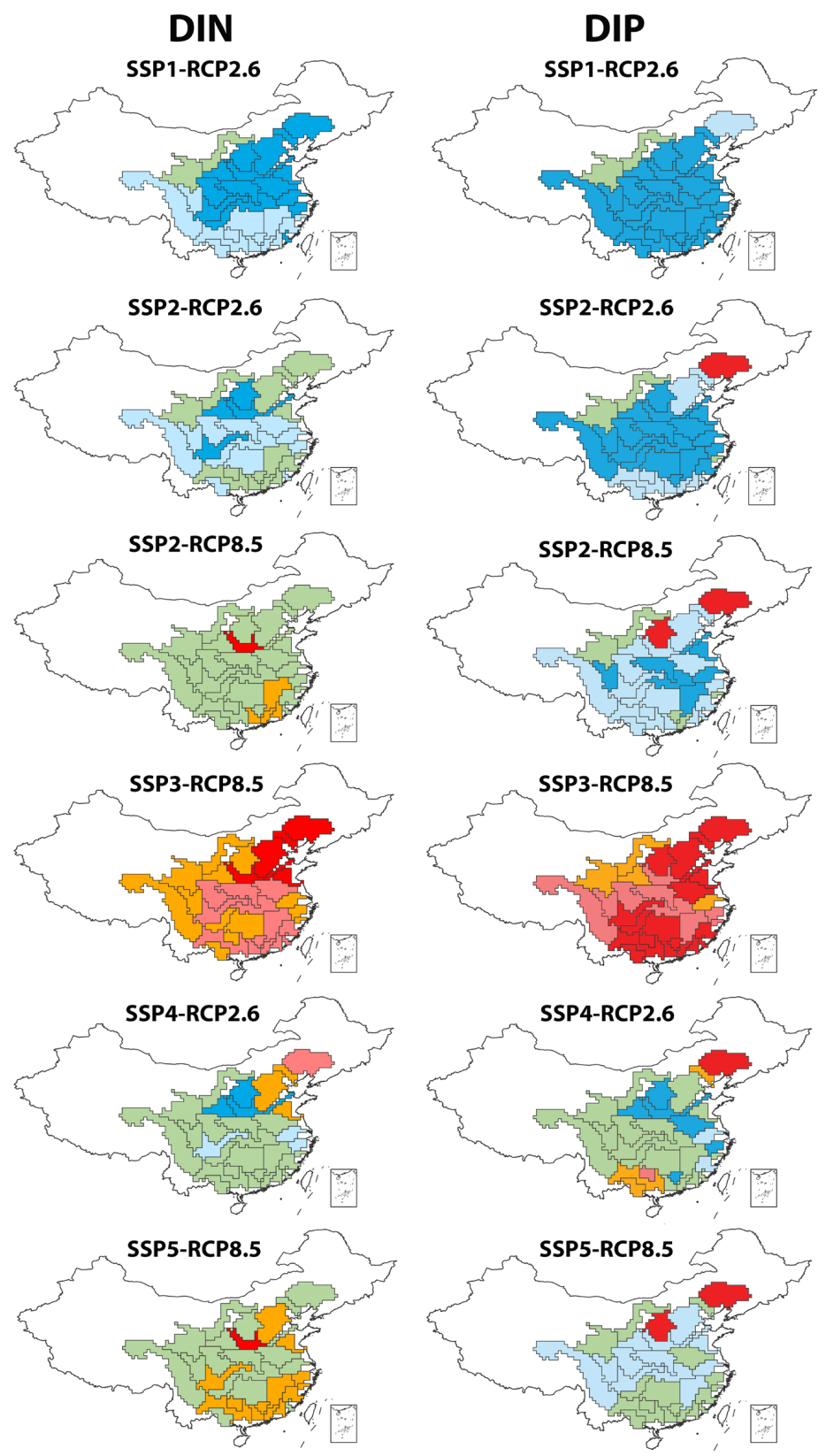

Changes in river export of DIN and DIP between 2012 and 2050

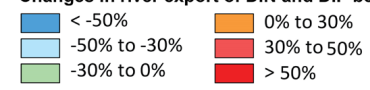

Figure 5.7 Changes (\%) in river export of dissolved N (DIN) and P (DIP) by sub-basins of the Chinese rivers between 2012 and 2050 for six SSP-RCP scenarios. SSPs are the Shared Socioeconomic Pathways. RCPs are the Representative Concentration Pathways. Details on the SSP-RCP scenarios are in Section 5.2.3. The names and locations of the rivers are in Figure 5.1, and Figures A.4-A.6 in Appendix IV. 


\section{ICEP}

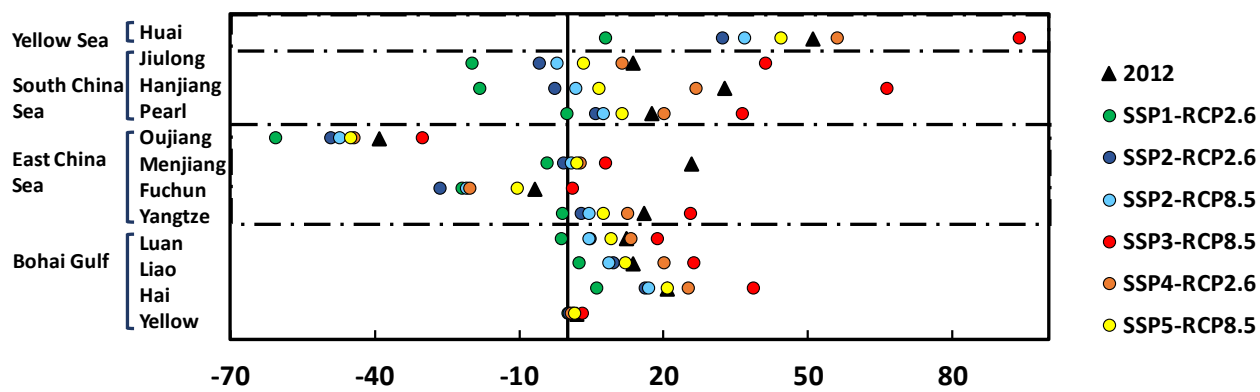

Figure 5.8 Indicator for Coastal Eutrophication Potential (ICEP, kg C-eq. $\mathrm{km}^{-2}$ day-1) for the Chinese rivers in 2012 and 2050 for six SSP-RCP scenarios. Positive ICEP values indicate high risks for coastal eutrophication. Negative ICEP value indicates low risks for coastal eutrophication. The approach to quantify ICEP is described in Section 5.2.2. SSPs are the Shared Socio-economic Pathways. RCPs are the Representative Concentration Pathways. Details on the SSP-RCP scenarios are in Section 5.2.3.

\subsubsection{Role of socio-economic change: hotspots for pollution}

We identified hotspots where $\mathrm{N}$ and $\mathrm{P}$ losses to rivers are higher than in other regions at the gridded $\left(0.5^{\circ} \times 0.5^{\circ}\right)$ scale for China. We compared changes in hotspots for $\mathrm{N}$ and $\mathrm{P}$ losses to rivers between 2012 and 2050 for the SSP scenarios (Figure 5.9, Figure C.4 in Appendix IV). We found that socio-economic change has a large impact on hotspots of $\mathrm{N}$ and $\mathrm{P}$ losses to rivers, and thus has a large impact on river export of TDN and TDP. In SSP3 (with high challenges towards sustainability), the number of hotpots for both diffuse (agriculture) and point (agriculture and human waste) sources increase considerably. In 2012, up to 0.9 million $\mathrm{km}^{2}$ of area (367 grids, $24 \%$ of the total river drainage area) in the river basins were identified as hotspots for $\mathrm{N}$ or $\mathrm{P}$ losses to rivers from the selected sources. The area of hotspots increases by 10-76\% (36-280 grids) for N losses, and by 3096\% (109-354 grids) for P losses from different sources in SSP3 compared to 2012 (Figure 5.9, Figure C.4 in Appendix IV). This results in increasing river export of TDN and TDP in SSP3 (Figure 5.4, Section 5.3.2).

In the SSP1 and SSP2 scenarios (focusing on more sustainability), the number of hotspots decreases for $\mathrm{N}$ and $\mathrm{P}$ losses from diffuse (agriculture) and point (agriculture and uncollected human waste) sources between 2012 and 2050. In these two scenarios, 13$68 \%$ (46-249 grids) of the hotspots for $\mathrm{N}$, and 20-68\% (75-245 grids) of the hotspots for P in 2012 are identified as nonhotspot area in 2050 (Figure 5.9, Figure C.4 in Appendix 
IV). This explains the relatively low river export of nutrients to seas in SSP1 and SSP2 (Figure 5.4, Section 5.3.2). However, the number of hotspots for $\mathrm{N}$ and $\mathrm{P}$ losses to rivers from sewage systems (collected human waste) increases in SSP1 and SSP2 as a result of a higher fraction of the population with sewage connections (Figure 5.9, Figure C.4 in Appendix IV).

The number of hotspots for $\mathrm{N}$ and $\mathrm{P}$ losses from agriculture and human waste in SSP4 and SSP5 either increase or decrease depending on the SSP storylines (Figure 5.9, Figure C.4 in Appendix IV). This explains the trends in river export of nutrients (Figure 5.4, Section 5.3.2). For example, the increases in hotspots for $\mathrm{N}$ and $\mathrm{P}$ losses to rivers from direct discharge of manure explain the relatively high river export of DON and DOP in SSP4 in 2050 . 


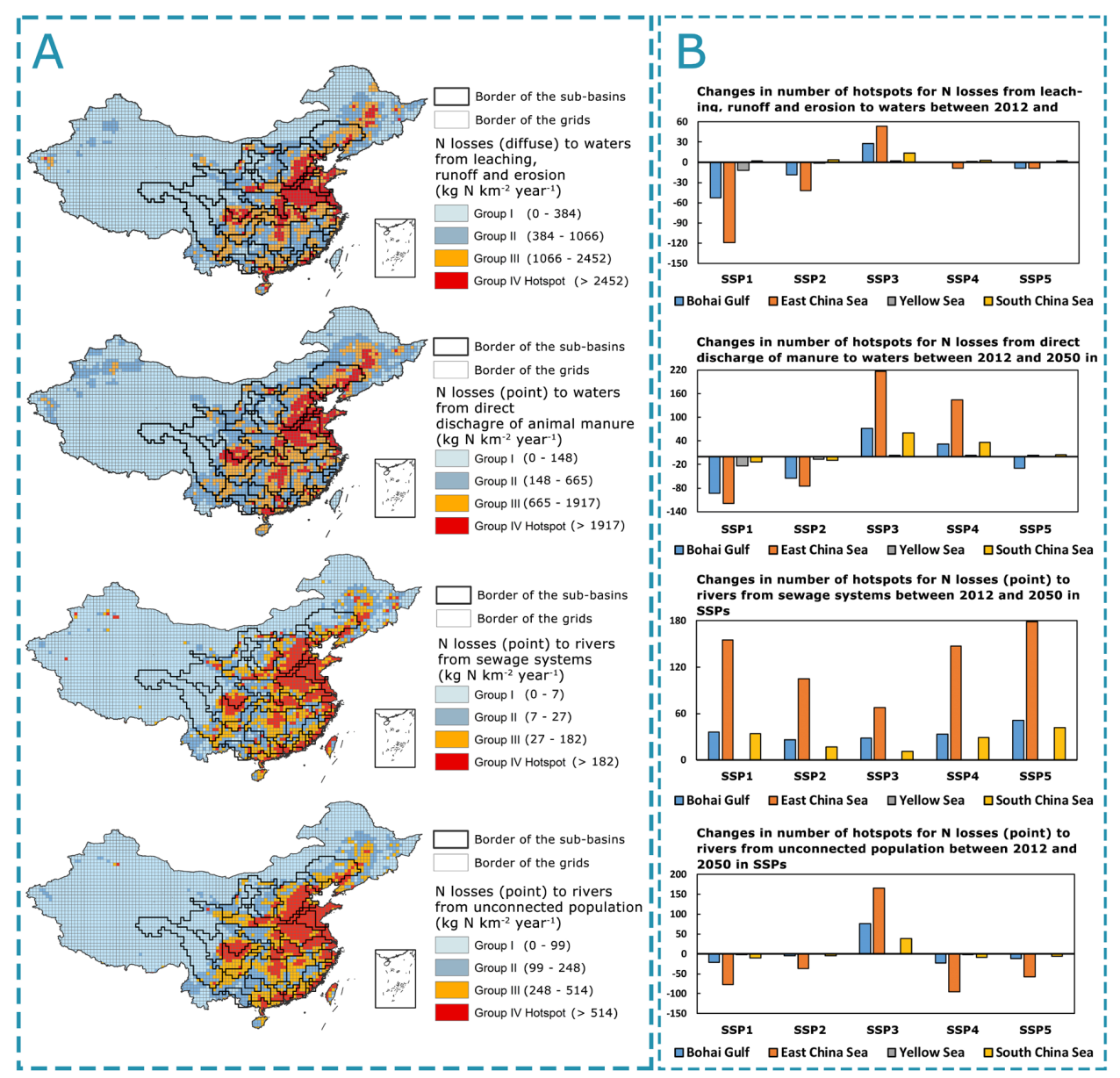

Figure 5.9 (A) Hotspots for N losses to waters (including rivers and groundwater) from leaching, runoff and erosion, and from direct discharge of animal manure, and hotspots for $\mathrm{N}$ losses to rivers from sewage systems and from unconnected population (discharge of uncollected human waste that is not connected to sewage systems) in 2012; (B) changes in the number of hotspots in the rivers that drain into the Bohai Gulf, East China Sea, Yellow Sea and South China Sea between 2012 and 2050 for the five SSPs. The hotspots were identified on the gridded scale following the approach described in Section 5.2.4. SSPs are the Shared Socio-economic Pathways. Details on the SSPs scenarios are in Section 5.2.3. 


\subsubsection{Role of climate change}

Climate change will impact surface and subsurface runoff, and river discharge patterns at different orders of magnitude (depending on the RCP, global climate model and hydrological model used). These hydrological changes can impact retentions of nutrients on land and in rivers, as well as nutrient runoff, and thus affect river export of nutrients to seas. For example, in China, natural river discharge by most of the sub-basins may increase (up to 21\%) in RCP8.5 (assuming severe climate change) and decrease (up to 68\%) in RCP2.6 (assuming lower climate change) between 2012 and 2050 (Figure B.8 in Appendix IV). The impacts of these hydrological changes on river export of nutrients are discussed below.

We found that river export of TDN and TDP in SSP3-RCP8.5 is 3-16 times that in SSP1RCP2.6 (Figure 5.4). This difference, however, cannot be completely attributed to climate change, since SSP1 and SSP3 differ in the assumed socio-economic change. To better understand the impacts of climate change alone on future river export of nutrients, we compared scenarios SSP2-RCP2.6 (low climate change) and SSP2-RCP8.5 (severe climate change). Our analysis indicates that climate change may increase nutrient inputs to seas considerably: nutrient export by the 12 Chinese rivers is higher in SSP-RCP8.5 than in SSP-RCP2.6. The difference is $24 \%$ for TDN and $16 \%$ for TDP (Figure 5.4). This is explained by the lower retentions of nutrients on land and in rivers resulted from the higher runoff and river discharge in RCP8.5 compared to RCP2.6 (Figures B.7, B.8, and C.6 in Appendix IV).

The impacts of climate change on river export of nutrients differ largely among sub-basins and nutrient forms. For most sub-basins except for Toudaoguai (sub-basin of Yellow River), Liao and Luan rivers, river export of DIN in 2050 in SSP2-RCP8.5 is 5-525\% higher than that in SSP2-RCP2.6. This range is 3-192\% for DON, 5-275\% for DIP, 3-174\% for DOP. River export of nutrient forms in SSP2-RCP8.5 is slightly lower (up to 9\%) than in SSP2RCP2.6 for Toudaoguai, Liao and Luan sub-basin (rivers). This is explained by the lower river discharge in SSP3-RCP8.5 than in SSP3-RCP2.6 in these sub-basins (Figures B.7 and B.8 in Appendix IV). 


\subsection{Discussion}

\subsubsection{Comparison with measurements and other studies}

We compared the MARINA 2.0 modeled loads of DIN, DIP, TDN, and TDP with measurements (Figure D.1 in Appendix IV). Based on the comparison, we assessed the model performance using three indicators: Pearson' coefficient of determination $\left(\mathrm{Rp}^{2}, 0\right.$ 1), the Nash-Sutcliffe efficiency (NSE, $-\infty-1$ ) and the root mean square error to the standard deviation of measured data (RSR, $0-+\infty$ ) (see Section D in Appendix IV for details). According to the performance ratings from Moriasi et al. (2007), our model shows a good performance with the indicators: Rp2 is 0.85 , NSE is 0.72 , RSR is 0.53 .

We also compared our modeled results with other modeling studies for the Yangtze River (Li et al., 2011a; Liu et al., 2018a; Tong et al., 2017; Yan et al., 2010). Yan et al. (2010) and Li et al. (2011a) modeled DIN and DIP exports to coastal waters from the Yangtze River in 2003. Liu et al. (2018a) estimated river export of TN and TP by Yangtze in 2010, while Tong et al. (2017) quantified the source attribution of riverine TN and TP export by Yangtze from 2006 to 2012. The comparison shows that our modeled DIN (1544 kton) export by the Yangtze River is comparable with the study of Yan et al. (2010) (1611 kton of DIN). We modeled higher DIP (128 kton) than Li et al. (2011a) (22-25 kton of DIP) knowing that Li et al. (2011a) did not include direct discharge of manure to rivers in their calculations. Our modeled TDN (2374 kton) and TDP (344 kton) is lower than the estimates of TN (5708) and TP (371) by Liu et al. (2018a). One reason for this difference is that we did not include the particulate forms in this study. Another reason is that Liu et al. (2018a) considers other sources that are not included in MARINA 2.0 such as aquaculture, vegetation in floodplains and industrial wastewater. Comparing the TDN and TDP loads to available measurements (Table D.1 in Appendix IV), our modeled result is closer to the measurements. Results of this study and Tong et al. (2017) both show that the contribution to river export of nutrients increases from upstream to downstream subbasins of Yangtze River. Tong et al. (2017) conclude that non-point sources are important for $\mathrm{N}(36 \%$ of TN) and $\mathrm{P}$ ( $63 \%$ of TP) export by the river. This is different from our results showing that point sources are dominant for DON, DIP, and DOP (see Section 5.3.1) because Tong et al. (2017) did not consider direct discharge of manure to rivers as a point source. Based on the above, we consider the MARINA 2.0 model acceptable for quantifying 
river export of nutrients (DIN, DON, DIP, and DOP) by sources, at the sub-basin scale for China.

Although our modeled results compare reasonably well to the measurements and other modeling studies, we need to realize our model has also uncertainties. Uncertainties in our model are related to the model structure, model inputs and parameters, and scenarios for the future. Uncertainties in the model structure are related to simplifications of nutrient flows from land to seas. The MARINA approach has been validated for large rivers (the Yellow, Yangtze, Pearl, Huai, Hai, and Liao rivers) (Strokal et al., 2016a) and several lakes (Lake Taihu, Dianchi, Baiyangdian and Guanting) in China (Li et al., 2019b; Wang et al., 2019a; Yang et al., 2019). These studies indicate an acceptable performance for MARINA 1.0 for quantifying river export of nutrients.

Uncertainties also exist in model inputs and parameters. Most of the inputs were taken from country statistics (e.g., GDP, sewage treatment plants), peer-reviewed papers, as well as published models (e.g., NUFER and VIC), which we believe, are the most reliable sources for China (see Figure A.1 and Table A.2 in Appendix IV). The MARINA approach (Strokal et al., 2016a) was developed based on the Global NEWS-2 model (Mayorga et al., 2010). The Global NEWS-2 model has been calibrated and validated for rivers worldwide (Amin et al., 2017; Mayorga et al., 2010; Pedde et al., 2017; Strokal and Kroeze, 2013) and for large Chinese rivers (the Yellow, Yangtze and Pearl) (Strokal et al., 2016a). We tested the sensitivity of model outputs to changes in selected model inputs and parameters (Figures E.1-E.4 in Appendix IV). Our modeled river exports of DIN, DON, DIP, and DOP for 2012 are relatively sensitive to changes in river discharge, direct discharge of animal manure and manure excretion. Most of these model inputs were derived from modeling studies published in peer-reviewed journals and published statistics, which are the most reliable datasets for China to our knowledge. Model inputs for river discharge were derived from the simulations by the VIC hydrological model (Van Vliet et al., 2016a) and Chinese Water Resources Bulletins (MWR, 2013) (see Table A.3 in Appendix IV for detailed approach). Our river discharge at the river mouth of the Yangtze $\left(859 \mathrm{~km}^{3}\right)$, Yellow $\left(78 \mathrm{~km}^{3}\right)$ and Pearl $\left(302 \mathrm{~km}^{3}\right)$ rivers are within the range of measurements (Table D.1 in Appendix IV). The model inputs for direct discharge of animal manure and manure excretion were calculated by the NUFER model based on Chinese statistics, peer-reviewed papers and surveys to farmers (Ma et al., 2012b; Wang et al., 2018b). 
There are also uncertainties related to the assumptions for future socio-economic developments and climate change in the SSPs-RCPs scenarios. For example, in the SSP1RCP2.6 scenario, we assumed some optimistic environmental management strategies (e.g., no direct discharge of manure), which may be very challenging to reach in the future. Despite these uncertainties, our scenarios provide a better understanding of future coastal water pollution for a wide range of possible changes in society and climate. This will help to identify potential improved water pollution management options for China. The optimistic environmental management strategies can be taken by the policymakers as a basis to identify feasible or optimal solutions.

\subsubsection{Implications for future policies}

This is the first assessment of river export of nutrients by Chinese rivers combining the impacts of SSPs (socio-economic change) and RCPs (climate change). Results show that future rivers and coastal waters in China may be considerably more polluted or considerably cleaner depending on the SSPs and RCPs. For example, by 2050, river export of total dissolved nitrogen (TDN) and phosphorus (TDP) is $52 \%$ and $56 \%$ higher than in 2012, respectively, in SSP3-RCP8.5. In contrast, river export of nutrients could be 56\% (TDN) and 85\% (TDP) lower in 2050 than in 2012 in SSP1-RCP2.6. In particular, manure management will have an important impact on future river export of nutrients. In addition to this, global climate change was found to be an important driver of coastal eutrophication in Chinese seas. We calculate considerable increases in nutrient export by rivers as a result of climate change. This indicates that effective policies aiming at reducing river and coastal water pollution in China do not only focus on improved nutrient management, but also on climate mitigation.

Improved nutrient management implies: 1) reducing discharge of manure, 2) improving nutrient use efficiencies in agriculture, and 3) improving sewage treatment in the middlestream and downstream sub-basins of rivers. Many technologies exist to support these policies. For example, animal manure can be recycled on cropland after treatment (e.g., composting) (Dong et al., 2018; Hou et al., 2017; Jia, 2014). Nutrient use efficiency of crop production can be improved by fertilizing crops according to their needs for nutrients (Zhang et al., 2012). Nutrients inputs to rivers from sewage can be much reduced by improved wastewater treatment (e.g., tertiary and quaternary treatment) (Van Puijenbroek et al., 2019). River export of N and P can be reduced by up to $90 \%$ depending 
on the nutrient forms in China between 2012 and 2050 for SSP1-RCP2.6, in which we assumed direct discharge of manure is completely prohibited. This confirms that current Chinese policies on restricting manure discharge (MOA, 2017) and increase manure recycling (MOA, 2015c) are effective and necessary to improve water quality in China.

Climate mitigation is a second way to reduce coastal eutrophication. As indicated above, coastal water pollution may increase as a result of climate change-induced changes in river discharge. This is indicated by the higher river export of TDN (24\% higher) and TDP (16\% higher) for the higher climate change scenario (SSP2-RCP8.5 versus SSP2-RCP2.6) in 2050. This is in line with Sinha et al. (2017), who concluded that climate changeinduced precipitation changes will likely increase future riverine nitrogen loading to seas in the United States (by about 20\%). Considerable increases in temperature and precipitation in the future are projected in China as a result of climate change (Lee et al., 2014; Sun et al., 2015a; Wang and Chen, 2014; Zhou et al., 2014a). For example, in Sun et al. (2015a), precipitation in China is projected to increase during the $21^{\text {st }}$ century by 13.39 $\pm 12.58 \%$ for RCP8.5. Thus to improve water quality in China, not only improved nutrient management in China is needed, but also climate change mitigation worldwide. Our most optimistic scenario (SSP1-RCP2.6) indicates that it is possible to reduce nutrient pollutions to relatively low levels to avoid coastal eutrophication, and to achieve climate mitigation and sustainable socio-economic development.

Global change impacts coastal water pollution not only in China, but also in other world regions. For example, Wang et al. (2019b) applied the MARINA 1.0 model to the Indus River and explored future trends in river export of N for selected SSPs and RCPs. A similar conclusion has been drawn by Wang et al. (2019b), who conclude that global change can increase coastal eutrophication at the river mouth of Indus. Suggestions for improved policies and technologies were given to the Indus basin based on the modeling results. Strokal et al. (2019) developed and applied the MARINA-Global model for rivers worldwide, and quantified river export of multiple pollutants (e.g., nutrients, plastics, and etc.) in 2010. Applying our approach of scenario analysis based on SSPs and RCPs using MARINA-Global can be the next step to analyze the impacts of global change on coastal water pollution, and to identify improved water pollution management options in other world regions. 


\subsection{Conclusions}

In this study, we described and applied the newly developed MARINA 2.0 model to analyze nutrient export by 12 Chinese Rivers for 2012 and 2050. We explore future trends in nutrient export by Chinese rivers as affected by socio-economic and climate change for the Shared Socio-economic Pathways (SSPs) and Representative Concentration Pathways (RCPs). In the following, we summarize our findings for China, which may also be relevant to other world regions.

Rivers and coastal waters in China are highly polluted in 2012. Rivers export 4854 kton of TDN, and 706 kton of TDP to Chinese seas. In the future rivers and coastal waters may be considerably more polluted or considerably cleaner than in 2012, depending on socioeconomic and climate changes. River export of TDN and TDP is projected to be $52 \%$ and $56 \%$ higher in 2050 than today, respectively, in the scenarios with high challenges towards sustainability (SSP3-RCP8.5 and SSP4-RCP2.6). River export of TDN and TDP is projected to be $56 \%$ and $85 \%$ lower in 2050 than today in the scenarios assuming more attention for sustainability (SSP1-RCP2.6, SSP2-RCP8.5, and SSP5-RCP8.5). Two-thirds of the rivers in 2050 in SSP1-RCP2.6 are projected to have low risks for coastal eutrophication.

Future nutrient management is expected to have a large impact on future river export of TDN and TDP, as indicated by our analysis of future hotspots. The largest hotspot area is found in scenario SSP3-RCP8.5. Our scenario analysis confirms that current policies in China on restricting manure discharge are effective in reducing nutrient water pollution. In addition to implementing and complying with current policies, we suggest further reductions in synthetic fertilizer use, increases in recycling of manure and improvements in wastewater treatment.

An important conclusion of our study is that global change can make coastal eutrophication control in China more difficult. Our scenarios indicate that climate change may increase nutrient pollution in rivers and coastal seas. Taking, for instance, the storylines for nutrient management in SSP2, we projected river export of TDN is $24 \%$, and TDP 16\% higher in SSP2-RCP8.5 (more climate change) than in SSP2-RCP2.6 (less climate change). Thus nutrient management may be less effective in a future world with severe climate change. Climate mitigation may, therefore, help to improve water quality in rivers and coastal waters of China, and likely of other countries, in the future. 


\section{Acknowledgments}

This study is funded by the National Key R\&D Program of China [project number 2016YFE0103100], the WIMEK (Wageningen Institute for Environment and Climate Research) fellowship [project number 5160957306], the KNAW (Koninklijke Nederlandse Akademie van Wetenschappen) project SURE+ [project number PSASA- E01], and the NWO Earth and Life Sciences (ALW) project [project numbers 863.14.008] and the NWO (Netherlands Organization for Scientific Research) project Multi Pollutant Modelling [project number 5160957330 DEELPRJ_1, 2016-2018]. Sources of the data sets used in this study are available in Table A.2 in Appendix IV. 



\section{Chapter 6. Exploring Nutrient Management Options to Increase Nitrogen and Phosphorus Use Efficiencies in Food Production of China}

\section{Abstract}

Low nitrogen (NUE) and phosphorus (PUE) use efficiencies in food production in China result in large losses of nitrogen $(\mathrm{N})$ and phosphorus (P) to the environment. The Chinese government formulated policies to increase the NUEs and PUEs. Recent policies aim for zero growth in synthetic fertilizer use after 2020 while ensuring food security. In this study we analyzed how current and improved nutrient management in China can affect future nutrient use efficiencies and nutrient losses from food production. The NUEs and PUEs of food production were quantified using the NUFER (NUtrient flows in Food chains, Environment and Resources use) model for 31 provinces and China in 2013, 2020 and 2050. Results show that national NUE (20\%) and PUE (24\%) in 2013 are low but vary largely among provinces (12-33\% for NUE, $10-53 \%$ for PUE). The N and P losses to the air (14 $\mathrm{Tg}$ year ${ }^{-1}$ of $\mathrm{N}$ ) and waters (12 $\mathrm{Tg}_{\text {year }}{ }^{-1}$ of N, 2 Tg year $^{-1}$ of P) are consequently high in 2013. Three scenarios were analyzed for 2020 and 2050 to explore future trends in NUEs and PUEs, assuming Business As Usual (BAU) trends, Zero Fertilizer (ZF) growth from 2020, and Improved Nutrient Management (INM). In the BAU scenario, the NUEs and PUEs roughly remain at their low 2013 levels, while nutrient inputs to agriculture are increasing. The losses to the air therefore increase by $37 \%$ for $\mathrm{N}$ and to waters by $40 \%$ for $\mathrm{N}$ and 48\% for P between 2013 and 2050. In the $Z F$ scenario, the NUEs and PUEs are a few percent higher than in $B A U$. The associated $\mathrm{N}$ and $\mathrm{P}$ losses to waters are 8-16\% lower than in $B A U$ because of increased recycling of manure to cropland, but still higher than in 2013. Improved nutrient management, as assumed in our INM scenario, may increase NUEs and PUEs to $33 \%$ and $59 \%$ in 2050. Meanwhile, $\mathrm{N}$ and P losses to waters in 2050 are $47 \%$ and $83 \%$ lower than in 2013 , and losses of $\mathrm{N}$ to the air $20 \%$ lower. We conclude that the policy aimed at zero growth in fertilizer use is a good start, but not very effective in reducing nutrient pollution in China. To substantially reduce $\mathrm{N}$ and $\mathrm{P}$ losses to the environment it is needed to improve nutrient management by not only reducing fertilization without yield losses, but also by improved manure management and animal production with lower nutrient excretion. 
Published as:

Wang M., Ma L., Strokal M., Chu Y., Kroeze C. Exploring nutrient management options to increase nitrogen and phosphorus use efficiencies in food production of China. Agricultural Systems 2018; 163: 58-72. 


\subsection{Introduction}

Nitrogen (N) and phosphorus (P) are essential elements for food production (Galloway et al., 2004). The use of $\mathrm{N}$ and $\mathrm{P}$ in agriculture has been increasing very fast in China since the 1980 s to feed the large population. According to FAO (2015b), synthetic fertilizer use in China is the highest worldwide, and almost twice that in the United States (US). Meanwhile China is becoming a large livestock producer. More than $50 \%$ of the global pork production, and 40\% of eggs took place in China in 2005 (FAO, 2015a; Scanes, 2007). However, nutrients are not used very efficiently in Chinese agriculture: the N and P use efficiencies (NUE and PUE) are very low because of poor nutrient management (Bai et al., 2014b; Bai et al., 2013; Liu et al., 2008; Ma et al., 2012b). Ma et al. (2010) concluded that the NUEs and PUEs of Chinese food production in 2005 was much lower than in European countries (EU) and the US.

As a result of this, the losses of $\mathrm{N}$ and $\mathrm{P}$ to the environment are large, causing air and water pollution. Agricultural greenhouse gas emissions (e.g., $\mathrm{N}_{2} \mathrm{O}$ ) contributed $17 \%$ to the national emissions from China (Chai et al., 2013; IEA, 2007; Nayak et al., 2013). Discharge of $\mathrm{N}$ and $\mathrm{P}$ to rivers results in eutrophication in many Chinese rivers (e.g., Yangtze, Yellow and Pearl rivers) and seas such as the Bohai Gulf, Yellow sea and South China sea (Liu et al., 2009; Müller et al., 2008; Qu and Kroeze, 2010; Strokal et al., 2014a; Strokal et al., 2014b; Sumei et al., 2008; Xie et al., 2014). This pollution will likely continue to increase in the future, as a result of the food demand by a growing population in China (Alexandratos and Bruinsma, 2012).

Since the early 2000s, the Chinese government has been recognizing these environmental challenges in food production (Zheng, 2013). There are several policies and regulations for sustainable agricultural production. For example, a "Discharge Standard of Pollutants for Livestock and Poultry Breeding" has been introduced in water polluted areas since 2003 to reduce pollution from livestock production (MEP, 2001). Another example is the so-called "Double High Agriculture" project, aiming for high yield of crops, and high nutrient use efficiency. This project is being implemented by the government, scientists, and farmers in Chinese agriculture (Chen et al., 2011; Fan et al., 2008; Fan et al., 2009; Zhang et al., 2011). Recently, the "Zero Growth in Synthetic Fertilizer Use from 2020" policy was introduced by the ministry of agriculture in China to increase nutrient use efficiencies, and to reduce pollution from agriculture (MOA, 2015c). It includes specific 
reduction targets for synthetic fertilizer use, manure recycling, and nutrient management for the period 2015 - 2020 (MOA, 2015b). Clearly, the Chinese government demonstrates willingness to move to a more sustainable food production.

The effectiveness of nutrient management strategies for Chinese agriculture is not well studied. Existing studies typically assess selective nutrient management practices, but not the effects of more integrated policies (Chai et al., 2013; Ermolieva et al., 2009; Huang et al., 2015; Ju et al., 2009; Ma et al., 2013a; Ma et al., 2013b; Nayak et al., 2013; Qu and Kroeze, 2012; Sims et al., 2013; Zhang et al., 2015). There are no integrated assessments of current policies as done for some other countries (Oenema et al., 2009; Velthof et al., 2014). Thus, a study that evaluates the effectiveness of current policies in China in improving $\mathrm{N}$ and $\mathrm{P}$ use efficiencies, and reducing nutrient pollution is needed. This would show us whether the current Chinese policies are effective. And if current policies are not effective, what would be needed for sustainable food production in the future.

In this study, we aim to analyze how current and improved nutrient management in China can affect future nutrient use efficiencies and nutrient losses from food production in all 31 provinces. To this end, we analyzed three scenarios for 2020 and 2050. These scenarios are assuming Business As Usual (BAU) trends, Zero Fertilizer (ZF) growth from 2020 representing current nutrient management, and Improved Nutrient Management (INM) in China. We updated the NUFER model to the year 2013 in order to quantify the current situation of NUEs and PUEs, and $\mathrm{N}$ and $\mathrm{P}$ losses from agriculture, and to implement the scenarios. This way, we can evaluate the effectiveness of current policies, and help to formulate nutrient management strategies to ensure food security with low environmental pollution in China.

\subsection{Method}

\subsubsection{Study area}

We analyzed the $\mathrm{N}$ and $\mathrm{P}$ use efficiencies of food production including crop and animal production for all 31 Chinese provinces and China (Figure 6.1). The 31 provinces are located in eight regions: North China, Northeast China, East China, Central China, South China, Southwest China, Plateau and Northwest China (Figure 6.1). Most crop production is in the eastern regions (North China, Northeast China, East China, Central China, South China, Southwest China), where more than $15 \%$ of the total land area is cultivated (Table 6.1). Animal production is more intensive in North China, Northeast China, East China, 
Central China, Southwest China, and Northwest China (Table 6.1). For example, the total number of animals in North China in 2013 (more than 30 million livestock units) was twice that in South China (less than 16 million).

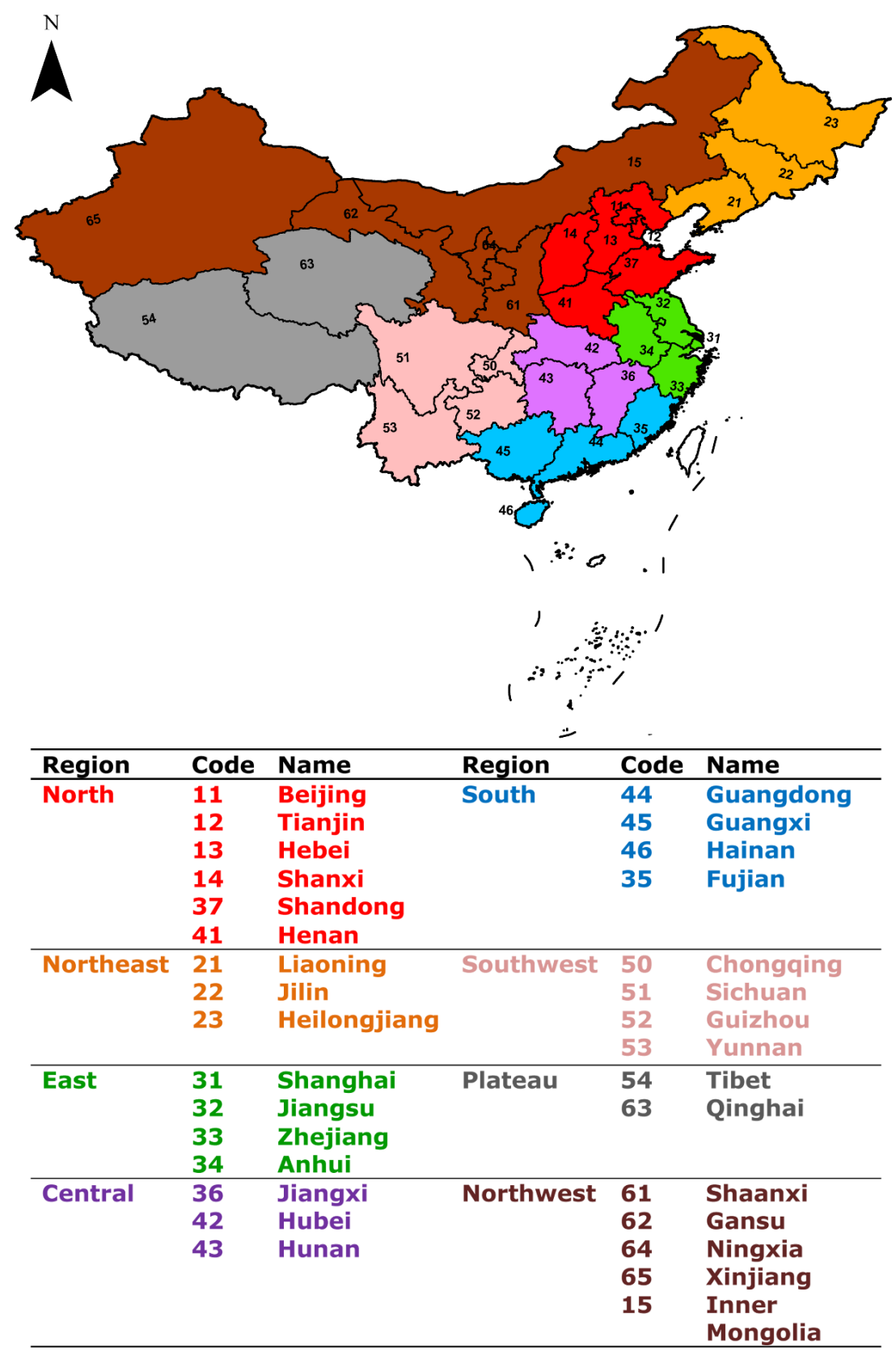

Figure 6.1 Overview of all 31 provinces in China, located in eight regions: North China, Northeast China, East China, Central China, South China, Southwest China, Plateau, and Northwest China. 


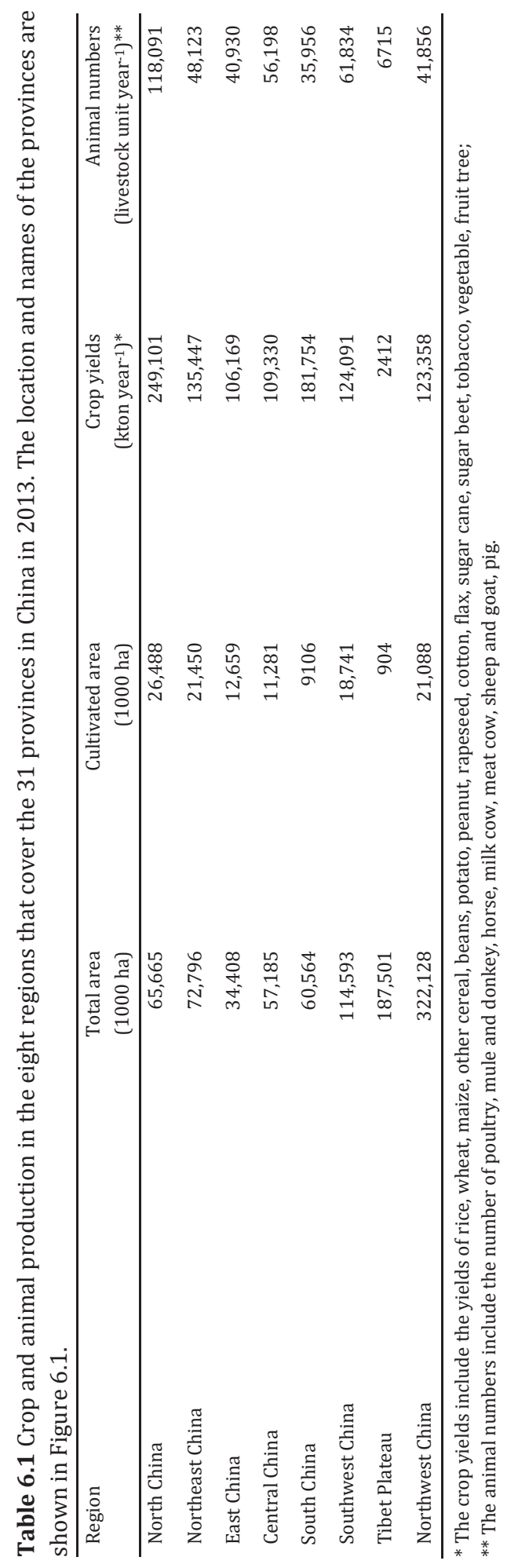




\subsubsection{NUFER}

We used the NUFER (NUtrient flows in Food chains, Environment and Resources use) model to quantify the $\mathrm{N}$ and $\mathrm{P}$ use efficiencies of food production for 2013, 2020 and 2050 (Ma et al., 2010; Ma et al., 2012b). NUFER was developed to quantify nutrient flows in the food chain of China from 1980 to 2005, and in 2030. This model also quantifies nutrient flows in the food chain of all 31 provinces in China in 1980 and 2005. NUFER has been widely applied to quantify nutrient flows in the food chain (Bai et al., 2014b; Bai et al., 2013; Hou et al., 2013; Ma et al., 2013b; Qin et al., 2012) and as a basis for policies aimed at improved nutrient management in China (MOA, 2015c). The food chain in NUFER includes crop production, animal production, food processing, and food consumption. The nutrient import to, output from and cycling within the food chain are quantified by this model.

For this study, we updated NUFER to the year 2013 for 31 provinces in China. The model inputs of NUFER are divided into three categories: 1) human activities in food production and consumption; 2) transformation and portioning coefficients; 3) N and P content and loss factors (Table 6.2). These input data for 2013 were collected from statistic yearbooks, survey reports and other studies (Ma et al., 2010; MOA, 2014; NBSC, 2014; Xu et al., 2015b).

NUFER quantifies $\mathrm{N}$ and $\mathrm{P}$ use efficiencies of food production (including crop and animal production) based on the nutrient flows in the food chain. Here the $\mathrm{N}$ and $\mathrm{P}$ use efficiencies are calculated as the $\mathrm{N}$ and $\mathrm{P}$ content in crop and animal products (e.g., yield of crops, animal meat) divided by total $\mathrm{N}$ and $\mathrm{P}$ inputs to crop and animal production (e.g., synthetic fertilizer, $\mathrm{N}$ deposition, animal feeds). Detailed information on the calculation method for $\mathrm{N}$ and $\mathrm{P}$ use efficiencies can be found in the equations in Box 6.1.

We also used NUFER to quantify the $\mathrm{N}$ and $\mathrm{P}$ losses to the air and aquatic systems from food production (including crop and animal production) based on the nutrient flows in the food chain (Ma et al., 2010; Wang et al., 2018b). The N losses to the air are the emissions of ammonia $\left(\mathrm{NH}_{3}\right)$ and nitrous oxide $\left(\mathrm{N}_{2} \mathrm{O}\right)$ from agriculture. The denitrification product dinitrogen $\left(\mathrm{N}_{2}\right)$ is also calculated in NUFER, but not considered here because it is not a pollutant. The $\mathrm{N}$ and $\mathrm{P}$ losses to aquatic systems (surface and ground waters) include surface runoff, leaching, and erosion of $\mathrm{N}$ and $\mathrm{P}$ from cropland, and direct discharge of animal manure to rivers. 
Table 6.2 The NUFER model inputs and their sources in 2013 for 31 provinces.

\begin{tabular}{lll}
\hline Model input & Examples & Data sources \\
\hline $\begin{array}{lll}\text { Human activity in food production and } \\
\text { consumption }\end{array}$ & $\begin{array}{l}\text { Crop yields; } \\
\text { Animal numbers; } \\
\text { Population }\end{array}$ & (MOA, 2014; NBSC, 2014) \\
Transformation and partitioning coefficients & $\begin{array}{l}\text { Ratio of straw to crop yield; } \\
\text { Ratio of edible part in animals }\end{array}$ & (Ma et al., 2010; Xu et al., \\
& & \\
2015b) & \\
N and P content and loss factors & N and P content in compound & (Ma et al., 2010) \\
& fertilizer; & \\
\hline
\end{tabular}

Box 6.1 Equations to quantify nitrogen use efficiency (NUEca) and phosphorus use efficiency (PUEca) in food production (crop and animal production) by the NUFER model. The equations are modified from (Ma et al., 2012b).

\begin{tabular}{|c|c|c|}
\hline NUECa or PUEca & $=\left(\right.$ Oca $_{\text {main product }} /$ Ica $) \times 100 \%$ & (1) \\
\hline Oca ${ }_{\text {main product }}$ & $=O c_{\text {crop product }}-I a_{\text {crop }}+O a_{\text {main product }}$ & (2) \\
\hline$O c_{\text {main product }}$ & $=O c_{\text {crop product }}$ & (3) \\
\hline Oamain product & $=O a_{\text {meat }}+O a_{e g g}+O a_{\text {milk }}+O a_{\text {fish }}+O a_{b y \text {-products to food }}$ & (4) \\
\hline Ica & $\begin{aligned}= & I c_{f e r t i l i z e r}+I c_{\text {human waste }}+I c_{\text {wet deposition }}+I c_{\text {dry deposition }}+I c_{\text {seed }}+ \\
& I c_{f i x a t i o n}+I c_{\text {irrigation }}+I c_{\text {straw residues }}+I c_{\text {crop by-products }}+ \\
& I a_{\text {crop by-products }}+I a_{\text {animal by-products }}+I a_{\text {kitchen residues }}+I a_{\text {other feed }}\end{aligned}$ & (5) \\
\hline \multicolumn{3}{|l|}{ Where, } \\
\hline Ica & Input of $\mathrm{N}$ or $\mathrm{P}$ to crop and animal production & \\
\hline Oca & Output of $\mathrm{N}$ or $\mathrm{P}$ from crop and animal production & \\
\hline Ic & Input of $\mathrm{N}$ or $\mathrm{P}$ to crop production & \\
\hline Ia & Input of $\mathrm{N}$ or $\mathrm{P}$ to animal production & \\
\hline Oc & Output of $\mathrm{N}$ or $\mathrm{P}$ from crop production & \\
\hline Oa & Output of $\mathrm{N}$ or $\mathrm{P}$ from animal production & \\
\hline$I c_{\text {wet deposition }}$ & Input of $\mathrm{N}$ via atmospheric wet nitrogen deposition & \\
\hline$I c_{d r y}$ deposition & Input of $\mathrm{N}$ via atmospheric dry nitrogen deposition & \\
\hline$I C_{\text {fertilizer }}$ & $\begin{array}{l}\text { Input of } \mathrm{N} \text { or } \mathrm{P} \text { via synthetic fertilizer including single and } \\
\text { compound fertilizer }\end{array}$ & \\
\hline$I c_{\text {manure }}$ & Input of $\mathrm{N}$ or $\mathrm{P}$ via animal manure application on land & \\
\hline IC $C_{\text {human waste }}$ & Input of $\mathrm{N}$ or $\mathrm{P}$ via human waste application on land & \\
\hline$I C_{\text {seed }}$ & Input of $\mathrm{N}$ or $\mathrm{P}$ via seed & \\
\hline$I c_{\text {fixation }}$ & Input of $\mathrm{N}$ via biological nitrogen deposition & \\
\hline I $c_{\text {irrigation }}$ & Input of $\mathrm{N}$ or $\mathrm{P}$ via irrigation & \\
\hline$I C_{\text {straw residues }}$ & Input of $\mathrm{N}$ or $\mathrm{P}$ via straw residues & \\
\hline$I C_{\text {crop by-products }}$ & Input of $\mathrm{N}$ or $\mathrm{P}$ via crop by-products from food processing & \\
\hline$I a_{\text {crop products }}$ & Input of $\mathrm{N}$ or $\mathrm{P}$ via grain and roots & \\
\hline$I a_{\text {straw }}$ & Input of $\mathrm{N}$ or $\mathrm{P}$ via straw & \\
\hline Ia grass & Input of $\mathrm{N}$ or $\mathrm{P}$ via grass from natural grass land & \\
\hline$I a_{\text {crop by-products }}$ & Input of $\mathrm{N}$ or $\mathrm{P}$ via crop by-products from food processing & \\
\hline$I a_{\text {animal by-products }}$ & Input of $\mathrm{N}$ or $\mathrm{P}$ via animal by-products from food processing & \\
\hline Ia $a_{\text {kitchen residues }}$ & Input of $\mathrm{N}$ or $\mathrm{P}$ via kitchen residues & \\
\hline Ia $a_{\text {otherfeed }}$ & Input of $\mathrm{N}$ or $\mathrm{P}$ via rough grazing and forests, and other source & \\
\hline
\end{tabular}




\subsubsection{Scenarios}

We analyzed three scenarios using NUFER to assess the effects of nutrient management on $\mathrm{N}$ and $\mathrm{P}$ use efficiencies and $\mathrm{N}$ and $\mathrm{P}$ losses. One scenario is a Business As Usual (BAU) scenario. The other two scenarios are alternatives to this BAU scenario, assuming Zero Fertilizer (ZF) growth from 2020, and Improved Nutrient Management (INM) (Table 6.3). The $B A U$ and $Z F$ scenarios were implemented for 2020 and 2050, while INM is only implemented for 2050. We choose the year 2020 because this is the current target year for the Chinese government to reach no growth in synthetic fertilizer use. And 2050 is chosen in line with the Millennium Ecosystem Assessment scenarios (Alcamo et al., 2005); for 2050 input data are readily available, for instance from FAO outlooks on food production (Alexandratos and Bruinsma, 2012).

\section{Business As Usual (BAU)}

In the $B A U$ scenario, crop and animal production in China is assumed to increase in order to meet the growing demand for food in the future. The expected larger population is assumed to have a dietary preference for animal products. The FAO projections for crop and animal production in Asian countries are used as a baseline for agricultural production in this scenario (Alexandratos and Bruinsma, 2012). The crop yields are assumed to increase by 8\% between 2013 and 2020, and by 28\% between 2013 and 2050 . Synthetic fertilizer application is assumed to increase by $6 \%$ in 2020 relative to 2013 and by $22 \%$ in 2050 . The total number of animals is calculated to increase by $13 \%$ in 2020 , and $59 \%$ in 2050 relative to 2013.

\section{Zero Fertilizer (ZF) growth from 2020}

The $Z F$ scenario was inspired by the current policy aimed at zero growth in synthetic fertilizer use from 2020 onwards (MOA, 2015c). This policy has specified goals for synthetic fertilizer use, animal manure recycling, and other nutrient management from 2014 to 2020 (Table S1 in Appendix V). Based on this, we assumed that the growth in synthetic fertilizer use will be zero from the year 2020. More manure (60\%) is assumed to be recycled and applied to cropland in 2020 and 2050 (Table 6.3).

\section{Improved Nutrient Management (INM)}

The INM scenario assumes further improvements in nutrient management (Table 6.3). Under this scenario, several improved nutrient management options are assumed to be 
implemented in food production in China from 2020 onwards. In 2020, China is assumed to reach the target of no growth in synthetic fertilizer use, as assumed in the ZF scenario. In INM, balanced fertilization ensures that $\mathrm{N}$ and $\mathrm{P}$ requirements of crops are met in a more efficient way (Ma et al., 2013a; Oenema et al., 2009). This is assumed to further reduce synthetic fertilizer use in 2050 without yield losses (S2 in Appendix V). As a result, in INM the synthetic fertilizer $\mathrm{N}$ input to crop production is reduced by $55 \%$ compared to $Z F$, and of $\mathrm{P}$ by $83 \%$ (Table 6.3). INM also assumes that the $\mathrm{N}$ and $\mathrm{P}$ excretion by animals is reduced by $20-30 \%$ in 2050 compared to $Z F$. This reduction is achieved by using improved animal feeding (less $\mathrm{N}$ and $\mathrm{P}$ in animal rations), and genetically modified animals that take up nutrients more efficiently (Oenema et al., 2009; Wang, 2005a; Wang, 2005b). Better manure management is incorporated to reduce $\mathrm{NH}_{3}$ emission from animal manure, and to increase the manure recycling to 90\% (Ma et al., 2013a; Zheng, 2013). 


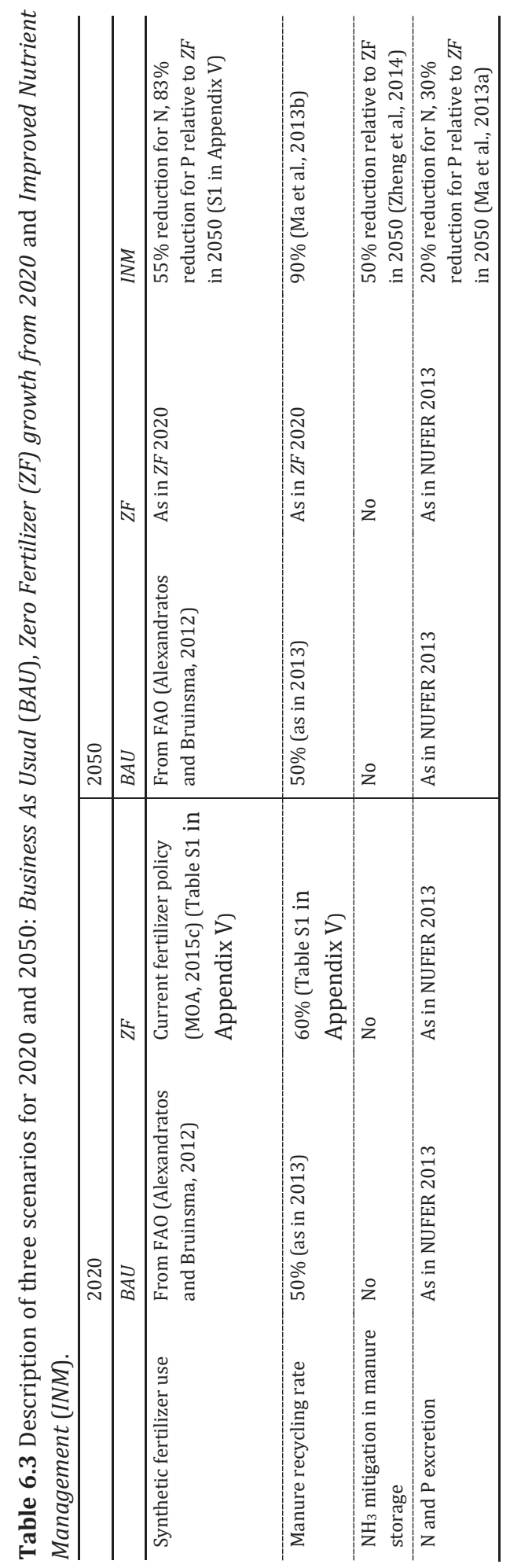




\subsection{Results}

\subsubsection{Results for 2013}

We calculate low N (20\%) and P (24\%) use efficiencies at the national level in 2013 (Figure 6.2). However, they vary largely among provinces: from 12 to $33 \%$ for NUE, and from 10 to 53\% for PUE (Figure 6.3). The N and P use efficiencies are generally higher in Northeast, North, East, Central and South China than in the other regions (See Figure 6.1 for locations). One of the reasons for the higher $\mathrm{N}$ and $\mathrm{P}$ use efficiencies in these regions is the more intensive and more productive agriculture than in the rest of China (MOA, 2014; Wang et al., 2018b). For example, in North China N and P use efficiencies are high as a result of productive maize and wheat double-cropping production systems (Liu et al., 2003; MOA, 2014; Wang et al., 2011b; Wu et al., 2015).

As a result of low $\mathrm{N}$ and $\mathrm{P}$ use efficiencies, the $\mathrm{N}$ and $\mathrm{P}$ losses to the air and aquatic systems are high in China in 2013. The crop and animal production together emitted $14 \mathrm{Tg}_{\text {year }}{ }^{-1}$ of $\mathrm{N}$ as $\mathrm{NH}_{3}$ and $\mathrm{N}_{2} \mathrm{O}$ (Figure 6.4). This is much higher than in the past: more than two times that in 1980 (Cai and Yan, 2011; Ma et al., 2012b). These losses to air vary among

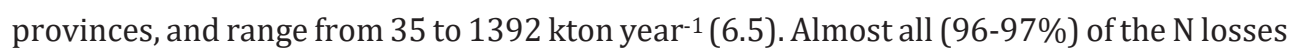
to the air are from $\mathrm{NH}_{3}$ emissions in crop and animal production (6.5). In some regions, such as Plateau, animal production dominates agriculture (Bai et al., 2013; MOA, 2014; NBSC, 2014). In such regions $\mathrm{NH}_{3}$ emissions from animal production exceed those from crop production. In other regions this is opposite. Meanwhile, $12 \mathrm{Tg}_{\text {year-1 }}$ of $^{\mathrm{N}}$ and $2 \mathrm{Tg}$ year $^{-1}$ of $\mathrm{P}$ are lost to aquatic systems from agriculture in 2013 (Figure 6.4). The losses of $\mathrm{N}$ to aquatic systems vary from 30 to 1113 kton year-1 $^{-1}$ (Figure 6.6), and that of $\mathrm{P}$ vary from 5 to 251 kton year $^{-1}$ (Figure 6.7). In most provinces $65-93 \%$ of these $\mathrm{N}$ losses are from synthetic fertilizers and direct discharge of animal manure (point source manure input) to waters (Figure 6.6). The point source manure input contributes $94-95 \%$ to total $\mathrm{N}$ inputs to aquatic systems because of the dairy and goat production in Plateau. More than half (54-97\%) of total P inputs to aquatic systems are from point source manure inputs, except for the Yunnan province (47\%) (Figure 6.7). Synthetic fertilizers contribute 2-38\% to the total P inputs to aquatic systems in the provinces. Our results clearly show that direct discharge of animal manure is the dominant source of nutrient pollution in surface waters in China. 


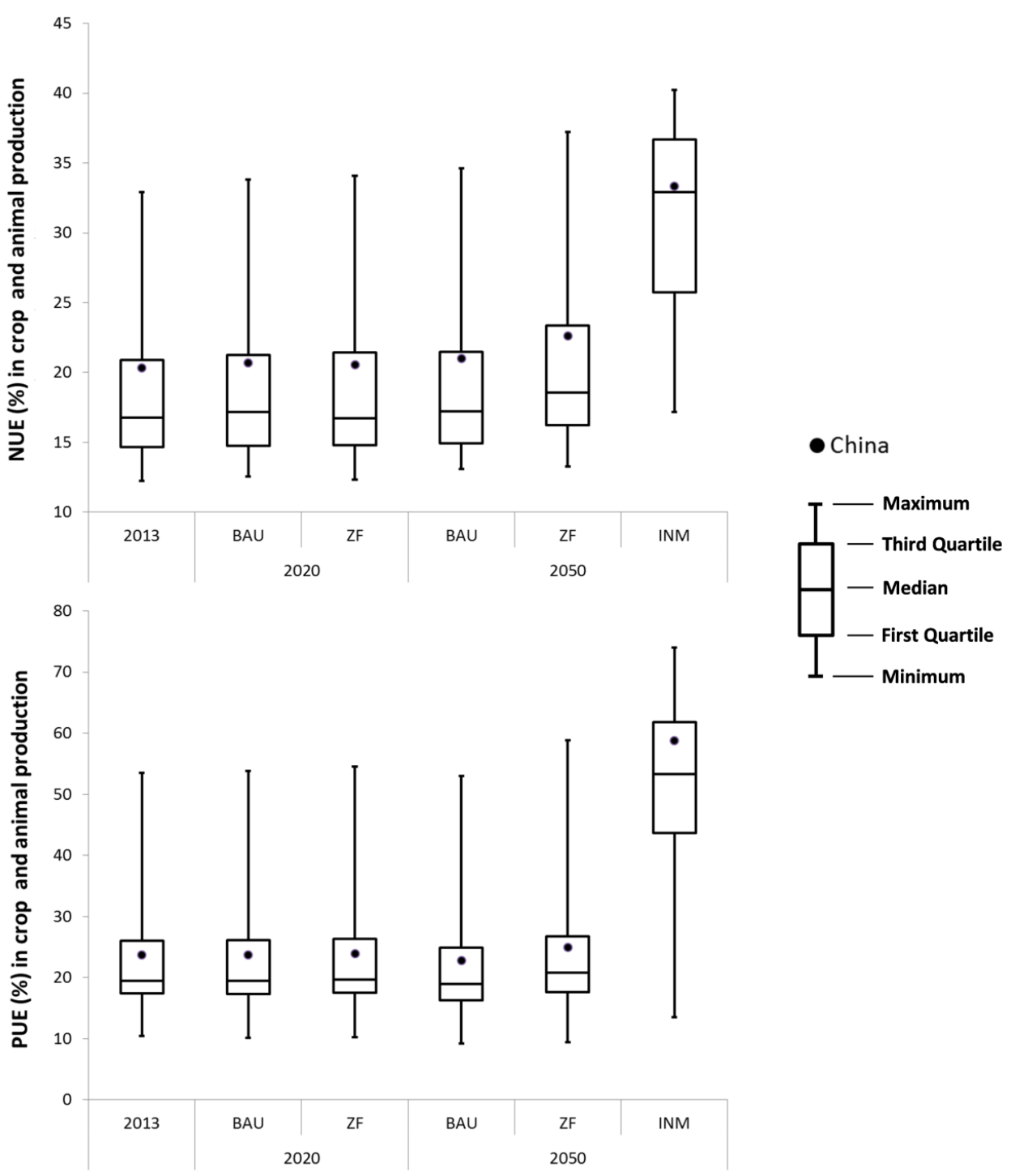

Figure 6.2 Nitrogen (NUE,\%) and phosphorus (PUE,\%) use efficiencies of crop and animal production for China in 2013, 2020 and 2050. The graph shows the national value for China (dot) and the quartile plot of values for 31 provinces. Scenarios for 2020 and 2050 assume Business As Usual (BAU) trends, Zero Fertilizer growth (ZF) and Improved Nutrient Management (INM). See Table 6.3 for scenario description. 

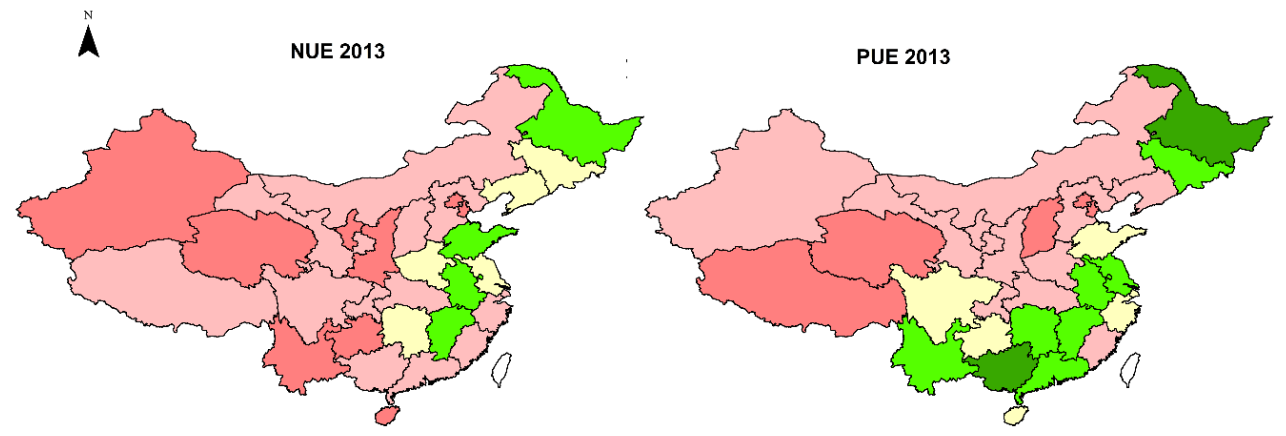

NUE (\%) and PUE(\%)

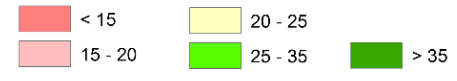

Figure 6.3 Nitrogen (NUE, \%) and phosphorus (PUE, \%) use efficiencies of crop and animal production for 31 provinces in China in 2013. 


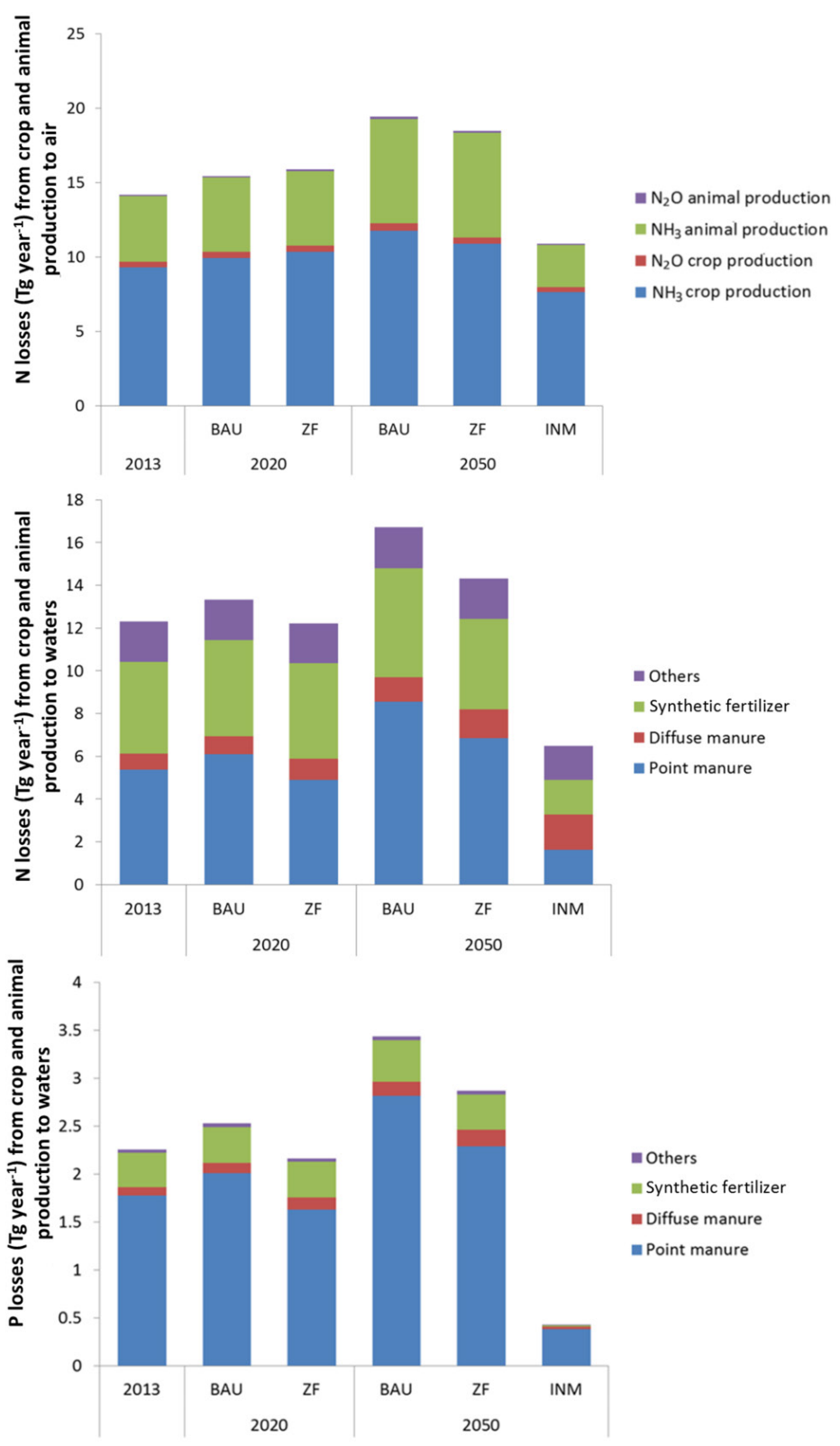

Figure 6.4 Total nitrogen $(\mathrm{N})$ and phosphorus $(\mathrm{P})$ losses $\left(\mathrm{Tg}^{\mathrm{year}}{ }^{-1}\right)$ to the air and aquatic systems and the share of sources (\%) in total losses for China in 2013, 2020 and 2050. Point manure refers to the $\mathrm{N}$ and $\mathrm{P}$ losses from direct discharging of animal manure to rivers. Diffuse manure is the $\mathrm{N}$ and $\mathrm{P}$ losses from manure that is applied to cropland. Others include $\mathrm{N}$ and $\mathrm{P}$ losses from cropland (e.g., atmospheric $\mathrm{N}$ deposition, biological $\mathrm{N}$ fixation, irrigation) except for synthetic fertilizer and diffuse manure (Wang et al., 2018b). Scenarios for 2020 and 2050 assume Business As Usual (BAU) trends, Zero Fertilizer growth $(Z F)$ and Improved Nutrient Management (INM). See Table 6.3 for scenario description. 

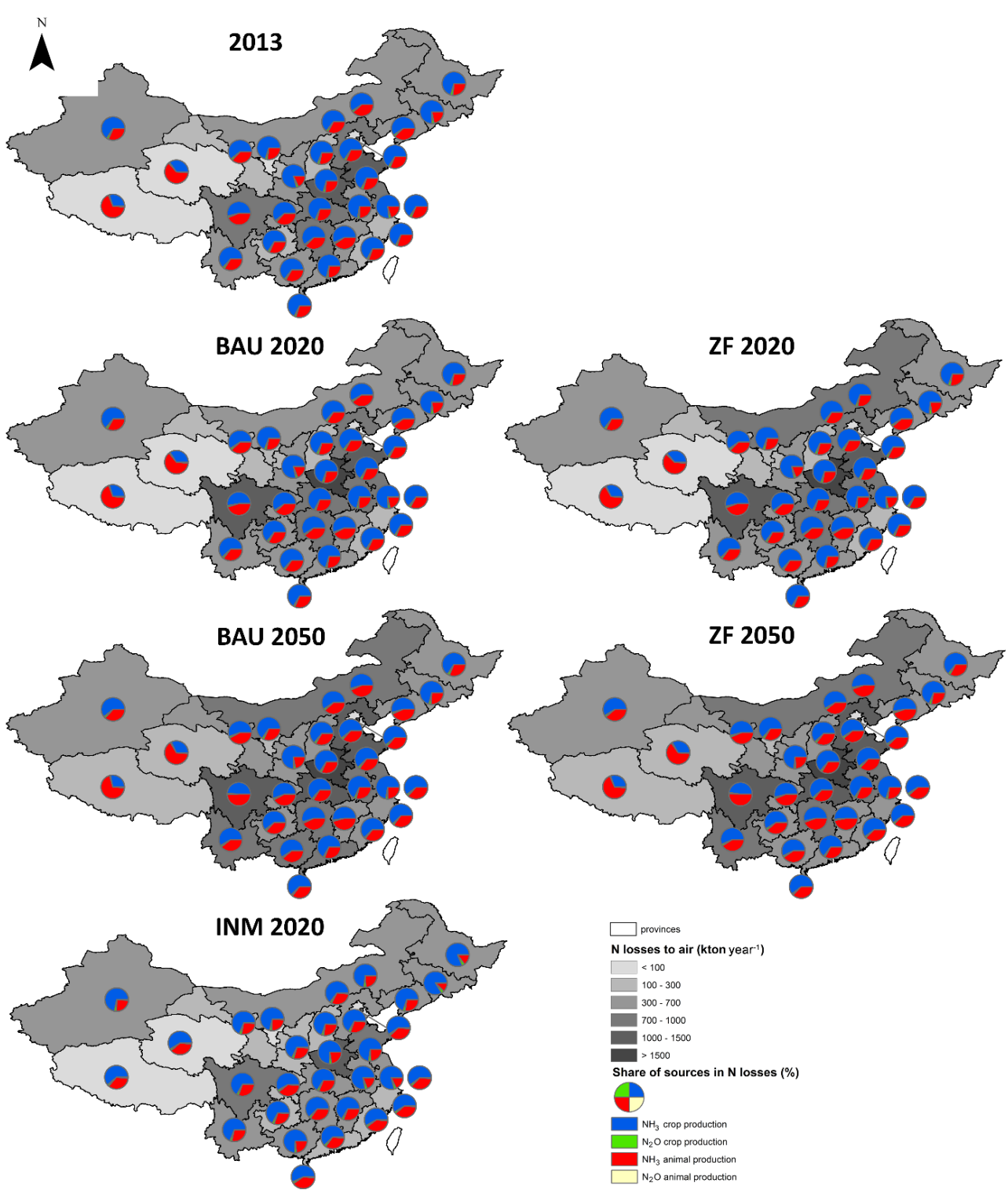

Figure 6.5 Nitrogen (N) losses to the air ( kton year $^{-1}$ ) and the share of sources (\%) in total losses for 31 provinces in 2013, 2020 and 2050. Scenarios for 2020 and 2050 assume Business As Usual $(B A U)$ trends, Zero Fertilizer growth (ZF) and Improved Nutrient Management (INM). See Table 6.3 for scenario description. 

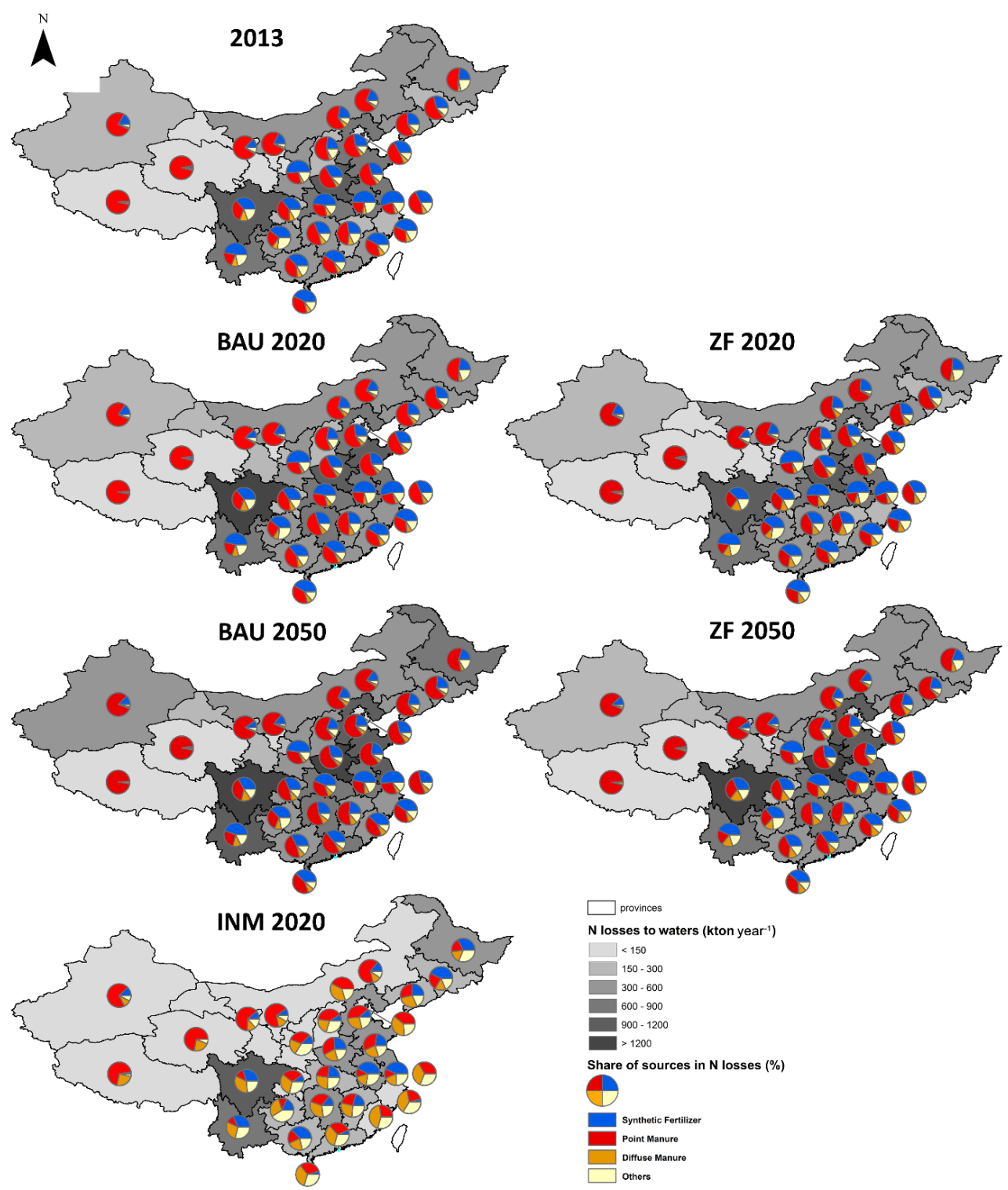

Figure 6.6 Nitrogen (N) losses to surface waters (kton year-1) and the share of sources (\%) in total losses for 31 provinces in 2013, 2020 and 2050. Point manure refers to the $\mathrm{N}$ and $\mathrm{P}$ losses from direct discharging of animal manure to rivers. Diffuse manure is the $\mathrm{N}$ and $\mathrm{P}$ losses from manure that is applied to cropland. Others include $\mathrm{N}$ and $\mathrm{P}$ losses from cropland (e.g., atmospheric $\mathrm{N}$ deposition, biological $\mathrm{N}$ fixation, irrigation) except for synthetic fertilizer and diffuse manure (Wang et al., 2018b). Scenarios for 2020 and 2050 assume Business As Usual (BAU) trends, Zero Fertilizer growth $(Z F)$ and Improved Nutrient Management (INM). See Table 6.3 for scenario description. 

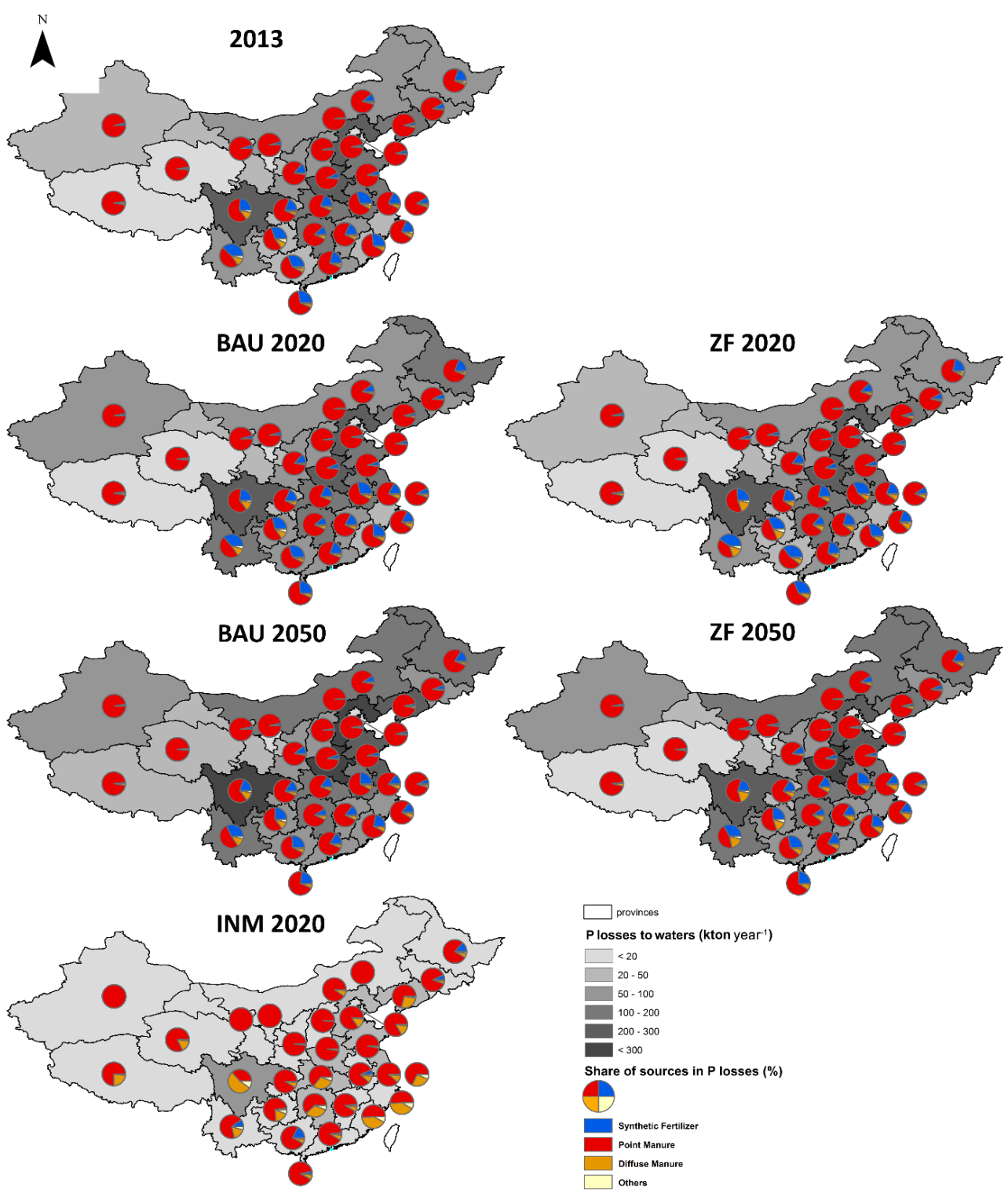

Figure 6.7 Phosphorus (P) losses to surface waters (kton year-1) and the share of sources (\%) in total losses for 31 provinces in 2013, 2020 and 2050. Point manure refers to the $\mathrm{N}$ and $\mathrm{P}$ losses from direct discharging of animal manure to rivers. Diffuse manure is the $\mathrm{N}$ and $\mathrm{P}$ losses from manure that is applied to cropland. Others include $\mathrm{N}$ and $\mathrm{P}$ losses from cropland (e.g., atmospheric $\mathrm{N}$ deposition, biological $\mathrm{N}$ fixation, irrigation) except for synthetic fertilizer and diffuse manure (Wang et al., 2018b). Scenarios for 2020 and 2050 assume Business As Usual (BAU) trends, Zero Fertilizer growth $(Z F)$ and Improved Nutrient Management (INM). See Table 6.3 for scenario description. 


\subsubsection{Scenario analysis: 2020 and 2050}

\subsubsection{Business As Usual (BAU)}

In the $B A U$ scenario, the $\mathrm{N}$ and $\mathrm{P}$ use efficiencies at the national level are projected to remain at their low 2013 levels in 2020 and 2050 (Figure 6.2). The changes in these use efficiencies between 2013 and 2020 are small in most provinces (Figures 6.9 and 6.10). Slight increases are projected for Heilongjiang, Jiangsu and Anhui provinces, while a small decrease is projected for Qinghai province between 2013 and 2020. In the other provinces, $\mathrm{N}$ and $\mathrm{P}$ use efficiencies are very comparable during this period.

The changes in $\mathrm{N}$ and P use efficiencies between 2013 and 2050 vary among the provinces (Figures 6.9 and 6.10). The $\mathrm{N}$ use efficiencies are projected to slightly increase in Northeast, North, East and South China, and slightly decrease in Plateau between 2013 and 2050. In other regions, the $\mathrm{N}$ use efficiencies in 2050 are comparable with the level of 2013. The $P$ use efficiencies are projected to slightly decrease in most provinces during this period except for Henan, Anhui, Hubei, Shaanxi, Chongqing, Fujian, Guangdong and Xinjiang, where PUEs are comparable with that in 2013. The decreases in PUEs are related to the fast increasing P inputs (e.g., synthetic fertilizer and animal feeds) in agricultural production (Figure 6.8).

The associated N and P losses are consequently higher in 2020 and 2050 than in 2013. This results from the low $\mathrm{N}$ and $\mathrm{P}$ use efficiencies and the increasing $\mathrm{N}$ and $\mathrm{P}$ inputs to agriculture in 2020 and 2050 under BAU (Figure 6.8). The $\mathrm{N}$ and $\mathrm{P}$ losses to the air, therefore, increase by $9 \%$ for $\mathrm{N}$, and to aquatic systems by $8 \%$ for $\mathrm{N}$ and $12 \%$ for $\mathrm{P}$ between 2013 and 2020 (Figure 6.4). Larger increases are projected for the period 20132050: a 37\% increase in $\mathrm{N}$ emissions to the air, and 36\% (N) and 52\% (P) to aquatic systems (Figure 6.4). The dominant sources of, and the variation in $\mathrm{N}$ and $\mathrm{P}$ losses among provinces in 2020 and 2050 are similar to that in 2013 because nutrient management is assumed to not change (Figures 6.5, 6.6 and 6.7). Direct discharge of animal manure to surface waters contributes slightly more to the $\mathrm{N}$ and $\mathrm{P}$ losses to waters in the future. This is caused by the increasing animal production to meet the meat demand in China. 


\subsubsection{Zero growth in synthetic Fertilizer use (ZF)}

In the $Z F$ scenario, the $\mathrm{N}$ and $\mathrm{P}$ use efficiencies for China do not change much between 2013 and 2020 (Figure 6.2). For 2050 we calculate $N$ and $P$ use efficiencies that are slightly higher than in 2013 (NUE increases from 20 to 23\%, and PUE from 24 to 25\%). This shows that policies aimed at a zero growth in fertilizers from 2020 are not very effective in increasing $\mathrm{N}$ and $\mathrm{P}$ use efficiencies. This result may seem surprising but can be explained by the inputs and outputs of $\mathrm{N}$ and $\mathrm{P}$ in food production underlying the $\mathrm{N}$ and $\mathrm{P}$ use efficiencies (Figure 6.8). The total output of $\mathrm{N}$ and $\mathrm{P}$ in food products in $\mathrm{ZF}$ is the same as in BAU. The total input of $\mathrm{N}$ and $\mathrm{P}$ in food production in $\mathrm{ZF}$ is comparable to that in 2020 and slightly lower than in 2050 in BAU. Although we assumed no increase in synthetic fertilizer use after 2020 in ZF, the reductions in N and P inputs are not large enough to make big changes in $\mathrm{N}$ and $\mathrm{P}$ use efficiencies. The higher manure recycling in ZF does not reduce the total $\mathrm{N}$ and $\mathrm{P}$ inputs to food production, because this is considered an internal nutrient exchange between crop and animal production.

The changes in N and P use efficiencies in ZF between 2013 and 2020, and between 2013 and 2050 vary among provinces. By 2020, the N use efficiencies increase in Northeast, North, East and South China, while in the other regions they remain comparable to 2013 or decrease slightly (Figure 6.9). The $P$ use efficiencies in 2020 are roughly the same as in 2013 with a slight increase in Heilongjiang and Yunnan provinces (Figure 6.9). Between 2013 and 2050, the $\mathrm{N}$ use efficiencies are projected to increase in most provinces except for Plateau, where NUEs decrease slightly (Figure 6.9). The largest increase from 33\% in 2013 to $37 \%$ in 2050 is calculated for Heilongjiang province. The P use efficiencies may either increase or remain at the level of 2013 in most provinces except for Liaoning province and Plateau region (Figure 6.9).

Our results show that $Z F$ reduces the $\mathrm{N}$ and $\mathrm{P}$ losses to the air and waters in 2020 and 2050 because of lower use of synthetic fertilizers and more recycling of animal manure on land. The variation in $\mathrm{N}$ and $\mathrm{P}$ losses among provinces in 2050 is similar to that in 2050 in $B A U$, because the nutrient management is assumed to improve to the same extent in all provinces in $Z F$. In 2020 the $Z F$ losses to the air are comparable to $B A U$. The $\mathrm{N}$ losses to the air are $5 \%$ lower in $Z F$ than in $B A U$ in 2050 (Figure 6.4). However, the $\mathrm{N}$ losses to the air in ZF 2050 are still higher than in 2013. In 2020, the losses of $\mathrm{N}$ to aquatic systems in $Z F$ are $8 \%$ lower than in $B A U$, and of $\mathrm{P} 14 \%$ lower (Figure 6.6). The $Z F$ scenario also 
manages to reduce the $\mathrm{N}$ and $\mathrm{P}$ losses to waters in 2020 to the level of 2013. In 2050, the $\mathrm{N}$ and $\mathrm{P}$ losses to waters in $Z F$ are $14-16 \%$ lower than in $B A U$, however higher than in 2013. The major source of the $\mathrm{N}$ and $\mathrm{P}$ losses to waters does not change much during the period 2013-2050 (Figures 6.9 and 6.10). Although considerable amounts of manure are recycled to land, direct discharge of manure remains an important source because of the increasing animal production. For example, the number of animals increases by almost 60\% between 2013 and 2050 in ZF (Table 6.3), while manure recycling only increases from 50 to $60 \%$. Thus, our $Z F$ scenario shows that the policies aimed at a zero growth in fertilizers from 2020 could help to reduce $\mathrm{N}$ and $\mathrm{P}$ losses to the air and waters in the future. However, nutrient pollution will still increase in the coming decades. Further nutrient management is needed to reduce the $\mathrm{N}$ and $\mathrm{P}$ losses to the environment.

\subsubsection{Improved Nutrient Management (INM)}

In the INM scenario, the NUE and PUE of crop and animal production in China are improved in 2050. The $\mathrm{N}$ use efficiency is projected to be $33 \%$, and $\mathrm{P}$ use efficiency to be $59 \%$ in 2050 under INM (Figure 6.2). This is much higher than in 2013 and higher than in $B A U$ and $Z F$ for 2050 . The $\mathrm{N}$ and $\mathrm{P}$ use efficiencies by province increase fast between 2013 and 2050. During this period, $\mathrm{N}$ use efficiencies increase $2-23 \%$ by province, and $\mathrm{P}$ use efficiencies increase 3-47\% (absolute change, see Figures 6.9 and 6.10).

As a result, the $\mathrm{N}$ and $\mathrm{P}$ losses to the air and aquatic systems are also reduced largely in $I N M$ by 2050 . N losses to the air and waters are $44 \%$ and $61 \%$ lower in INM than in $B A U$ in 2050, respectively (Figure 6.4). P losses to waters are 88\% lower (Figure 6.4). Our analysis also shows that in INM the N and P losses in 2050 are lower than in 2013. The losses of $\mathrm{N}$ to the air in 2050 in INM are 23\% lower than in 2013 (Figure 6.4). $\mathrm{N}$ and $\mathrm{P}$ losses to waters decrease as well, by $47 \%$ for $\mathrm{N}$ and $81 \%$ for $\mathrm{P}$ (Figure 6.4). This will largely reduce air and water pollution in China. The variation in $\mathrm{N}$ and $\mathrm{P}$ losses among provinces in 2050 in $I N M$ is similar to that in 2050 in $B A U$ because the nutrient management is assumed to improve to the same extent in all provinces in INM. The shares of sources in N and P losses are very different in the INM than in 2013 and the other two scenarios. This is related to higher manure recycling (90\%) and lower synthetic fertilizer use in INM (Table 6.3). For example, the share of point source manure inputs and synthetic fertilizers in total N losses to waters in INM 2050 is much lower (27-82\%) than in 2013 (65-96\%) (Figure 6.6). Synthetic fertilizer (on average 2\%) almost does not contribute to 
P losses to waters, while point manure (36-99\%) is still the dominant source of P losses to waters (Figure 6.7). This indicates that treatment of manure (e.g., anaerobic digestion that produces biogas) before discharge is needed to reduce the $\mathrm{N}$ and $\mathrm{P}$ pollution of water in China.

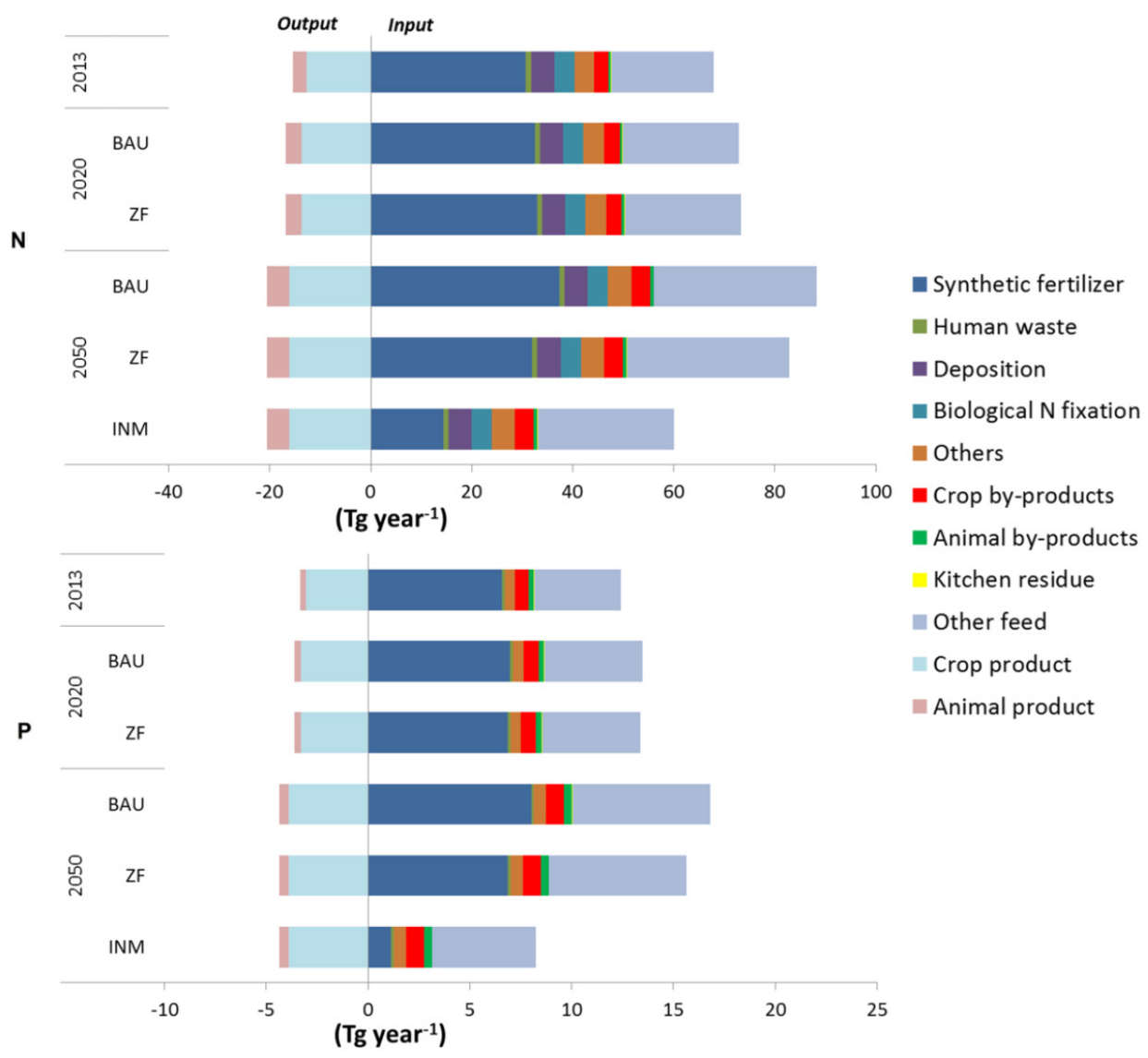

Figure 6.8 Input of nitrogen $(\mathrm{N})$ and phosphorus $(\mathrm{P})$ to, and output of nitrogen $(\mathrm{N})$ and phosphorus (P) via products from crop and animal production (Tg year-1) for China in 2013, 2020 and 2050. Scenarios for 2020 and 2050 assume Business As Usual (BAU) trends, Zero Fertilizer growth (ZF) and Improved Nutrient Management (INM). See Table 6.3 for scenario description. 

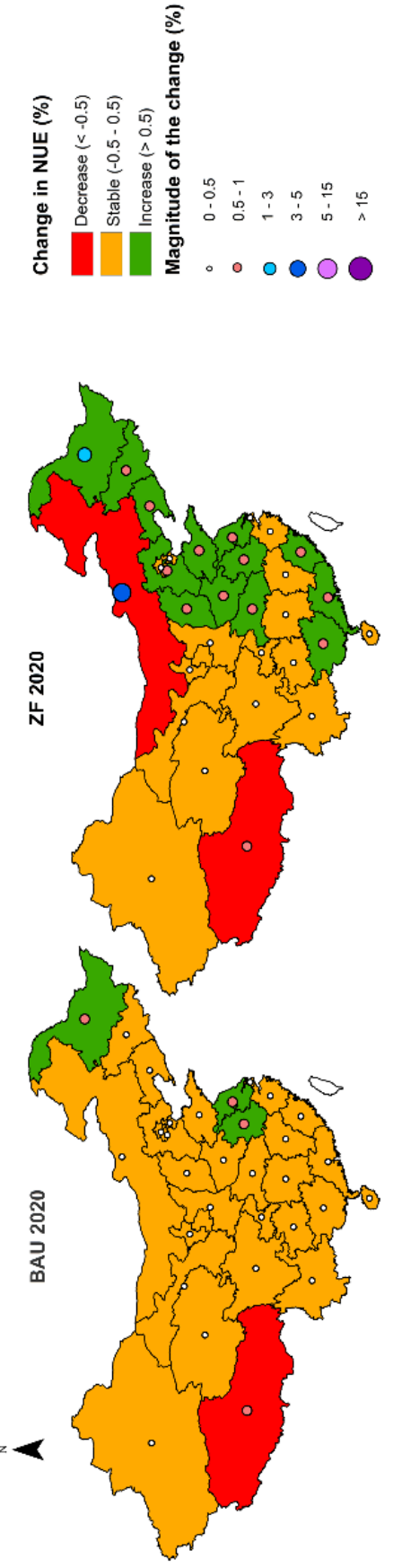

용 चี 峁 mi พิ ㄴํㅇ ¿ छี ํ ปั ฐัँ ํํㄹㄹ 窤 กิ ฐั๊ 온 $\Xi z \pi$ पू के 을 त्ञ ले 퐁 을 운 ปั
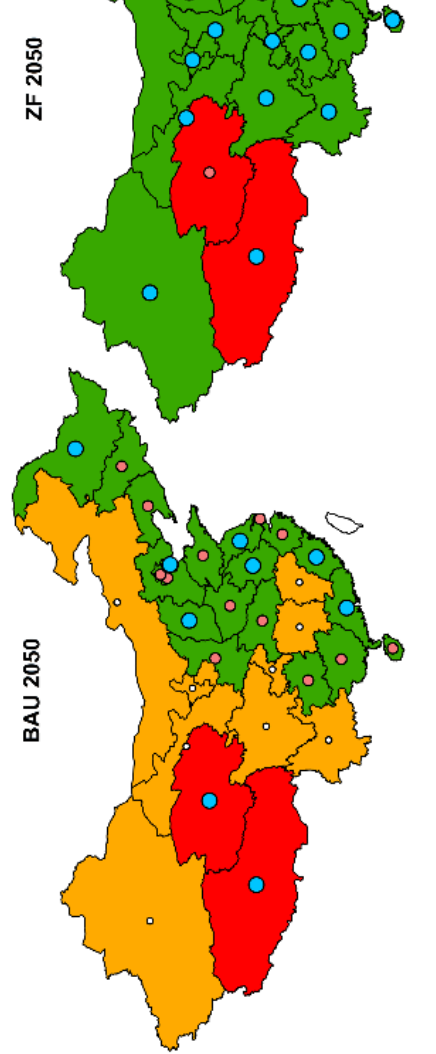

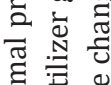

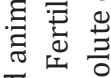
들 ڤ 윤 บ 넌 四㐫

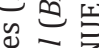
幽 胥 ฮิ 电要 ڤ્仓 ป 원 兄豆 ฮ

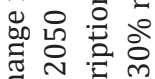
已 ర

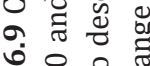
๑ 은 은 ํํㄹ 워

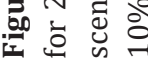



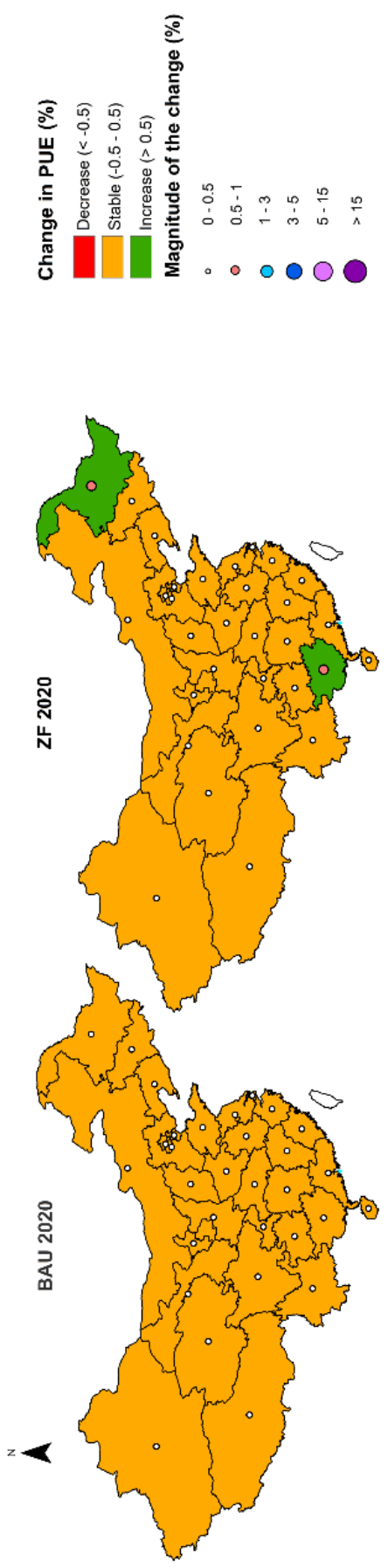

을 홍

สํํ

岕泀

它

กิ

范家

品

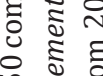

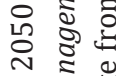

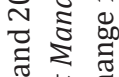

กับ

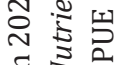

$\Xi \sum \frac{2}{\pi}$

\& 8

मี

है हี

के च

$\Xi \stackrel{0}{*}$

远

흥 范

ة.

똟

䒕

동

ㅇำ 원

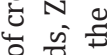

폼

氮焉

可

ํㅗㄴ

氙

is

范

है

究

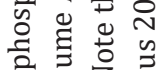

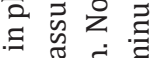

济

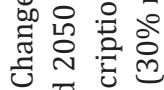

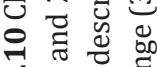

ํํ유을

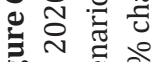

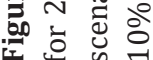




\subsection{Discussion}

\subsubsection{The need for improved nutrient management}

From the results, it is clear that we need to do more than the current policy to increase $\mathrm{N}$ and $\mathrm{P}$ use efficiencies, and thus to reduce $\mathrm{N}$ and $\mathrm{P}$ losses to the environment. This will require other and better nutrient management policies and techniques. The INM scenario is a good example of better nutrient management for Chinese food production. INM assumes techniques (e.g., Integrated Soil-Crop System Management by Zhang et al. (2011)) that increase the crop productivity and help to reduce fertilization without yield losses (Fan et al., 2008; Fan et al., 2009; Zhang et al., 2011). Improved manure management techniques can reduce the $\mathrm{N}$ and $\mathrm{P}$ losses from animal manure. For example, emissions of $\mathrm{NH}_{3}$ from manure storage can be reduced to a large extent (Brink, 2003; Hou et al., 2015; Zheng et al., 2014). More animal manure can be processed and applied on cropland to replace synthetic fertilizer rather than discharged directly to waters as a waste stream. This reduces water pollution. The use of higher-quality animal feeds and genetically modified animals could decrease the nutrient excretion (Bai et al., 2014b; Oenema and Tamminga, 2005; Wang et al., 2014a; Yang, 2013). In addition to the nutrient management in $I N M$, the treatment of animal manure before it is discharged to waters is also crucial to reduce water pollution. For example, anaerobic digestion of manure and nutrient recovery techniques can be used to produce energy, recover nutrients, and reduce the risk of water pollution by nutrients and chemicals (Nasir et al., 2012; Yilmazel and Demirer, 2011). In order to ensure food security in the future with lower environmental impact, all above improvement in fertilizer use, manure management and recycling, and feed quality are needed in Chinese agriculture. The feasibility of implementing the improved nutrient management options in our scenarios in China and the strengths and weaknesses of this study are discussed in Sections 6.4.2 and 6.4.3.

\subsubsection{Feasibility of the scenarios}

Results indicate that the INM scenario is an effective way to improve $\mathrm{N}$ and $\mathrm{P}$ use efficiencies of food production in China. It may reduce $\mathrm{N}$ and $\mathrm{P}$ losses to the environment. The feasibility of implementing this scenario can be discussed with respect to technical, practical and economical aspects. The technical feasibility of INM is high. Many technical measures to improve nutrient management in crop and animal production have already been applied in China and in other world regions such as Europe. For example, Integrated 
Soil-Crop System Management (ISSM) was developed by China Agricultural University to increase crop productivity and improve nutrient use efficiency (Zhang et al., 2011). This ISSM has been successfully applied to different cropping systems in China to ensure food security while reducing environmental pollution by nutrients (Chen et al., 2011; Zhang et al., 2012). The techniques of manure management that are applied in Europe (Oenema et al., 2009), such as low-emission manure application practices and low-protein feeding could be adjusted and applied in animal production in China. The practical feasibility of the INM scenario needs some more attention. Farmers in China need to be better educated to apply improved nutrient management in food production. Fortunately, the Chinese government is aware of this and is providing such education to farmers. For example, the "Double High Agriculture" project supported by the central government organizes master students from agricultural universities in China to guide farmers on managing nutrients, soil and water in their agricultural production activities (CAU, 2016). The economic feasibility depends on the costs and benefits of the INM scenario. (Zheng et al., 2014) summarized the costs and effectiveness of several manure management options in China, which helps to choose effective management options that are relatively cheap. Developing technical measures that are effective in managing nutrients in food production and also relatively cheap should be given more attention in the future. Summarizing, we conclude that implementing INM is technically feasible, can become practically feasible. To what extent it is economically affordable needs to be analyzed. But it is clear that INM includes options (e.g., ISSM) that are economically affordable.

\subsubsection{Strengths and weaknesses of this study}

As any other modeling study, our study has weaknesses. An important source of uncertainty in our scenario analyses is associated with model inputs and parameters that are based on assumptions. For example, in the $B A U$ scenario, we assume that the crop yields in all provinces of China will increase by the same percent between 2013 and 2020, and between 2013 and 2050. However, the increase in crop yield may differ among provinces in the coming decades as it also differed in the past (Ma et al., 2012b; MOA, 2014). We realize that this assumption may affect the results, but assumed that the effects would not influence the main message of our study. It should be noted that the absolute increase in crop yields differ among provinces in 2020 and 2050 in $B A U$. This is because crop yields vary among provinces in NUFER in 2013, for which we use the data from Chinese statistical yearbook that is the most reliable source of Chinese agricultural data 
that is available. Another example is that we assume that $90 \%$ of the collected animal manure is recycled to crop production in the INM scenario. However, in some provinces (e.g., Beijing and Fujian) the total $\mathrm{N}$ and $\mathrm{P}$ in recycled manure exceeds the amount of $\mathrm{N}$ and $\mathrm{P}$ required by crops. This means that in these provinces, we applied slightly more nutrients from manure to crop production than what crops need. However, the effect of this on the overall results is likely small because it happens only in a few provinces (four of the 31), and the extra amount is not large.

Despite the weaknesses, we believe that our study provides valuable results for future food production, and may help to reduce the environmental pollution in China. Our study has four main strengths. First, we used a nutrient model that allows quantifying $\mathrm{N}$ and $\mathrm{P}$ use efficiencies and the associated $\mathrm{N}$ and $\mathrm{P}$ losses to the environment in the food production level. This offers the possibility to look at nutrient use efficiencies and losses in the production of both crop and animal products, while other studies only focus on either crop or animal production (Bai et al., 2014b; Cui et al., 2010; Ma et al., 2008; Wang et al., 2014a). We think that crop and animal production are equally important in China because of the large production and consumption of meat, milk, and eggs (Bai et al., 2013; Schneider, 2011). Second, we assessed the effectiveness of current fertilizer policy on $\mathrm{N}$ and $\mathrm{P}$ use efficiencies in food production, and $\mathrm{N}$ and $\mathrm{P}$ losses to the environment. This was not done by others before. It allows policymakers and other stakeholders to evaluate the effectiveness of current policies in improving nutrient use efficiencies and in reducing environmental pollution. Third, we explored other improved nutrient management options for sustainable food production in China. Some of these options are well studied and applied in other world regions. For example, balanced fertilization was assessed by Oenema et al. (2009) in European countries. We believe that our results can be used as a good basis for better nutrient management in food production of China. Last but not least, we used the NUFER model that is well accepted in China. This model was developed for China in 2010 and has been widely applied to study nutrient flows in food production (Bai et al., 2014b; Bai et al., 2013; Hou et al., 2013; Ma et al., 2013b; Qin et al., 2012). The results of NUFER were also used by the policymakers to develop nutrient management policies in China (MOA, 2015c). This also makes the results of this study convincing to the policymakers and other stakeholders. 


\subsection{Conclusion}

In this study, the $\mathrm{N}$ and $\mathrm{P}$ use efficiencies of food production in China were quantified for the year 2013, and for future years (2020 and 2050). The related $\mathrm{N}$ and P losses to the environment were also calculated. Our analyses cover 31 provinces and China. We updated the NUFER model for 2013. We developed, implemented and analyzed three scenarios using NUFER to assess policies, and to explore future trends in N and P use efficiencies in 2020 and 2050. These three scenarios are named Business As Usual (BAU), Zero Fertilizer (ZF), and Improved Nutrient Management (INM).

Our analysis for 2013 shows that current $\mathrm{N}$ and P use efficiencies are low (20\% for NUE, and $24 \%$ for PUE). The N (12-33\%) and P (10-53\%) use efficiencies vary largely among provinces because of differences in agricultural production systems. Low N and P use efficiencies result in high $\mathrm{N}$ and $\mathrm{P}$ losses to the environment. Emissions from crop and animal production amounted to more than $14 \mathrm{Tg}_{\text {year }}{ }^{-1}$ of $\mathrm{N}$ as ammonia and nitrous oxide in 2013. Over $12 \mathrm{Tg}$ year $^{-1}$ of $\mathrm{N}$ and $2 \mathrm{Tg}$ year ${ }^{-1}$ of $\mathrm{P}$ are lost to aquatic systems.

In the BAU scenario, the NUEs and PUEs remain low (around the 2013 level). As a result, the $\mathrm{N}$ and $\mathrm{P}$ losses increase because of higher $\mathrm{N}$ and $\mathrm{P}$ inputs to agriculture to meet the increasing food demand. Losses of $\mathrm{N}$ to waters are projected to increase by $40 \%$, and of $\mathrm{P}$ by $48 \%$ between 2013 and 2050 . An increase of $37 \%$ is projected for $\mathrm{N}$ losses to the air.

Our result for the $Z F$ scenario indicates that the current policy aimed at zero growth in synthetic fertilizer use after 2020 is not very effective in increasing $\mathrm{N}$ and $\mathrm{P}$ use efficiencies in agriculture in China. In this $Z F$ scenario, the $\mathrm{N}$ and $\mathrm{P}$ use efficiencies are about the same as in the $B A U$ scenario for 2020. By 2050 the $\mathrm{N}$ and $\mathrm{P}$ use efficiencies are a few percents higher in $Z F$ than in $B A U$. Thus the new policy may only improve the $\mathrm{N}$ and $\mathrm{P}$ use efficiencies to a small extent. However, it may help to reduce $\mathrm{N}$ and $\mathrm{P}$ losses to waters because of the increased recycling of manure. The associated $\mathrm{N}$ and $\mathrm{P}$ losses to waters on the national level are 8-16\% lower than in BAU. However, these losses are still higher than in 2013. In contrast, the $\mathrm{N}$ losses to the air are not reduced much by these recent policies: $\mathrm{N}$ losses to the air are comparable in the $B A U$ and $Z F$ scenarios.

The INM scenario is more effective than $Z F$ in improving $\mathrm{N}$ and $\mathrm{P}$ use efficiencies and in reducing nutrient pollution. In the INM scenario, the $\mathrm{N}$ and $\mathrm{P}$ use efficiencies are projected to increase to $33 \%$ and $59 \%$, respectively, in 2050 . The $\mathrm{N}$ and $\mathrm{P}$ losses to the air and aquatic systems are much lower in INM than in 2013. The losses of $\mathrm{N}$ and $\mathrm{P}$ to waters are 
projected to be $47 \%$ and $83 \%$ lower than in 2013 . And the losses of $\mathrm{N}$ to the air are $20 \%$ lower than in 2013.

Our analysis indicates that the current fertilizer policy is a good start, but is not enough to improve $\mathrm{N}$ and $\mathrm{P}$ use efficiencies in China. It also has a limited effect on $\mathrm{N}$ and $\mathrm{P}$ losses from agriculture to the environment. Further improvements in nutrient management are required. In our INM scenario we accounted for several effective improvements including: 1) technologies to reduce fertilization without yield losses, 2) better manure management (e.g., more manure recycling, emission mitigation during storage), 3) animal production with lower $\mathrm{N}$ and $\mathrm{P}$ excretion (genetically modified animal species, higher quality feeds). We show that there is a large potential to improve NUEs and PUEs, and to reduce environmental pollution simultaneously. Treatment of animal manure before it is discharged to waters can further reduce nutrient pollution. We conclude that there is a need to develop effective nutrient management options in addition to the current policies.

\section{Acknowledgments}

We acknowledge Wageningen Institute for Environment and Climate Research (WIMEK) of Wageningen University, and Netherlands Organization for Scientific Research (NWO) for financial support. We also acknowledge the Chinese National Basic Research Program (2015CB150405), President's International Fellowship Initiative, PIFI of the Chinese Academy of Science (2015VEA025), the Hundred Talent Program of the Chinese Academy of Science for supporting this research. 



\title{
Chapter 7. Reducing Nutrient Pollution in Rivers and Coastal Waters in China: Implications for the Sustainable Development Goals
}

\begin{abstract}
Reducing nutrient pollution in rivers and coastal waters is important for China, given its commitments to the Sustainable Development Goals (SDGs). The aim of this study is to explore future scenarios that contribute to meeting SDGs 6 - "clean water and sanitation" and 14 - "life below water" from the perspective of nutrient pollution in China. We quantified total dissolved nitrogen (TDN) and phosphorus (TDP) inputs to six Chinese rivers, and river export of TDN and TDP to seas at the sub-basin scale in 2012 and 2050 for six future scenarios. This was done using the MARINA 2.0 (Model to Assess River Inputs of Nutrients to seAs) model. Indicators were identified for assessing SDGs 6 and 14. We first analyze a baseline scenario. Our results show that it is difficult to meet SDGs 6 and 14 in the baseline scenario. Nutrient pollution in rivers and coastal waters will remain at high levels until 2050. Close to $90 \%$ of the 26 sub-basins do not meet SDG 6 at their outlets. Six large rivers do not meet SDG 14, and have a high potential for coastal eutrophication at the river mouths in the baseline. Next, we analyze alternative scenarios. Results show that combing improved nutrient management in agriculture and sewage systems, healthy and efficient food consumption, as well as climate mitigation. This combination may be effective to meet SDGs 6 and 14. Results show that in 2050, all subbasins may meet SDG 6 for P pollution at their outlets, while $32 \%$ of these sub-basins meet SDG 6 for N pollution. Three of the six large rivers in this study may meet SDG 14 in 2050. Our alternative scenarios may also contribute to meeting other SDGs such as SDG 2 - "zero hunger", SDG 11 - "sustainable cities and communities", SDG 13 - "climate action" and others. This study may help in formulating future policies to ensure good water quality in rivers and coastal waters for SDGs 6 and 14, and to meet other related SDGs.
\end{abstract}

To be submitted:

Wang M., Kroeze C., Ma L. Reducing nutrient pollution in rivers and coastal waters in China: implications for the Sustainable Development Goals. To be submitted. 


\subsection{Introduction}

Reducing nutrient pollution in rivers and coastal waters is important for China given its commitments to the Sustainable Development Goals (SDG) under the United Nation's (UN) Agenda 2030 (United Nations, 2015). This holds in particular for SDG 6 - "Ensure access to water and sanitation for all" and SDG 14 - "Conserve and sustainably use the oceans, seas and marine resources". China started the implementation of the 2030 agenda for sustainable development in 2016 (GPRC, 2016). Improved nutrient management in sewage systems and agriculture is included in the national policies for meeting SDGs 6 and 14. However, based on the assessment by Stiftung (2018), with current implementations China is not on track towards meeting most of the SDGs in 2030. Significant challenges (e.g., fast population growth, show progress towards sustainable production and consumption) still exist that make it difficult to meet the SDGs, in particular, SDGs 6 and 14.

Food production and sewage discharge are important causes of water pollution in China. For example, food production has increased in the last decades at the expense of high nutrient application. Consumption of synthetic fertilizers in China has skyrocketed, increasing from <0.1 Mton in 1950 to 50 Mton in 2010 (Chen et al., 2018; NBSC, 2011). However, typically more than half of these nutrients are not used by crops and are lost to the environment, because of the low nutrient use efficiencies in Chinese agriculture (Ma et al., 2012b; Wang et al., 2018a; Yu et al., 2019). China currently has nearly 50\% of the worlds' pig population, but with poor manure management (Bai et al., 2014b; Bai et al., 2018b; NBSC, 2014). In 2012, about 5.5 kton of the $\mathrm{N}$ and 2 kton of the $\mathrm{P}$ in the manure was discharged to rivers without proper treatment (Wang et al., 2018b). The wastewater discharge from urban areas has also increased in China, to nearly 69.5 billion $\mathrm{m}^{3}$ in 2013 (MEP, 2013a). On average less than 70\% of this human waste was treated in wastewater treatment plants (MOHURD, 2012; MEP, 2013a).

The increasing amount of nutrients in rivers from agriculture and sewage has deteriorated water quality in China, posing threats to society and ecosystems (Bai et al., 2018a; Gu et al., 2013; Yu et al., 2019). Between 2000 and 2012, 28\% of groundwater samples over China exceeded the WHO's maximum contaminant level $\left(10 \mathrm{mg} \mathrm{N} \mathrm{L}^{-1}\right)(\mathrm{Gu}$ et al., 2013). In 2010, up to $36 \%$ of the river sections and $40 \%$ of the major lakes did not meet the quality criteria for drinking water sources (MWR, 2010). Excessive nutrients in 
rivers are further transported to seas, which has caused approximately 500 times harmful algae blooms in the coastal area of China between 2006 and 2012 (SOA, 2012). This situation is expected to worsen in the future under the expected socio-economic developments and climate change (Crespo Cuaresma, 2017; Howarth et al., 2006; Sinha et al., 2017; Strokal et al., 2016b; Wang et al., Under Review).

This study explores future scenarios that contribute to meeting SDGs 6 and 14 from the perspective of nutrient pollution in China. We quantified total dissolved N (TDN) and P (TDP) inputs to six Chinese rivers, and river export of TDN and TDP to seas in 2012 and 2050. The year 2050 was selected because meeting all SDGs in 2030 is challenging for developing countries (Nhamo et al., 2019; Stiftung, 2018; Van Puijenbroek et al., 2019). Six scenarios were analyzed for 2050. A baseline scenario (referred to as SSP5-RCP8.5) from Wang et al. (Under Review) was selected by combining one of the Shared Socioeconomic Pathways (SSP5) and one of the Representative Concentration Pathways (RCP8.5). This baseline reflects a future with a relatively high global warming. Five alternative scenarios were developed assuming improved sewage treatment (SE), improved nutrient use efficiencies in agriculture (AG), a combination of AG and SE $(\mathrm{SE}+\mathrm{AG})$, sustainable food consumption in addition to $\mathrm{AG}+\mathrm{SE}(\mathrm{AG}+\mathrm{SE}+\mathrm{SFC})$, climate mitigation in addition to $\mathrm{AG}+\mathrm{SE}+\mathrm{SFC}(\mathrm{AG}+\mathrm{SE}+\mathrm{SFC}+\mathrm{CLI})$. The scenarios were implemented in the MARINA 2.0 (Model to Assess River Inputs of Nutrients to seAs) model. Based on the results, we analyzed the contributions of the scenarios to meet SDGs 6 and 14. We also discussed the effects on other SDGs based on the assumptions in the scenarios. Our study ends with implications for future policies to reduce nutrient pollution in waters and to meet SDGs in China.

\subsection{Methodology}

\subsubsection{Study area}

We modeled nutrient pollution in rivers and coastal waters of six large rivers in China (Figure 7.1). These rivers are: the Liao, Hai and Yellow rivers draining into the Bohai Gulf; the Huai River draining into the Yellow Sea; the Yangtze River draining into the East China Sea; and the Pear River draining into the South China Sea. We selected these rivers because they contribute largely to the nutrient pollution in the coastal waters of China. According to Wang et al. (Under Review), these six rivers contribute by around $90 \%$ to river export of TDN and TDP to Chinese seas in 2012. The drainage basins of Yellow, 
Yangtze, and Pearl were divided into upstream, middle-stream and downstream subbasins following (Wang et al., Under Review). The names of the sub-basins are available in Figure A.1 in Appendix VI.

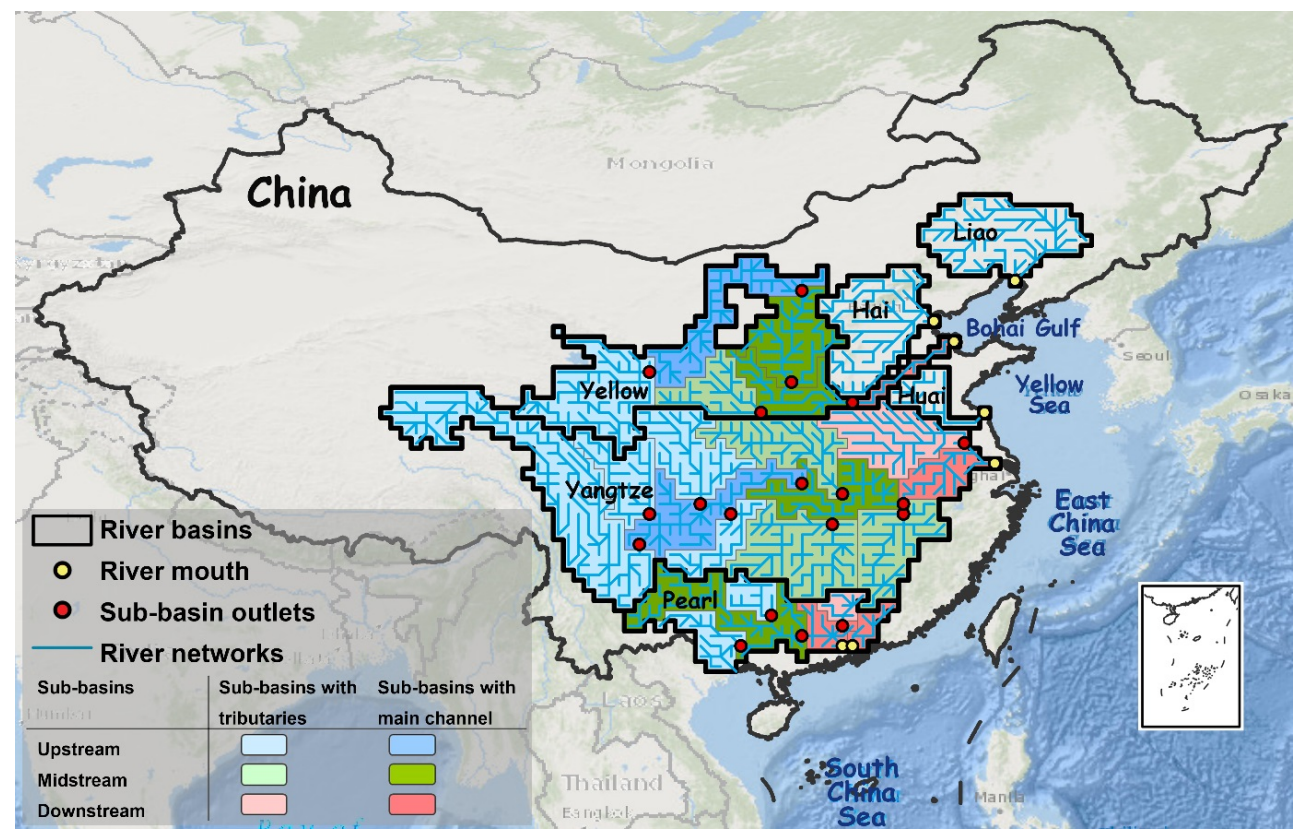

Figure 7.1 Overview of the study area. We consider six large Chinese rivers: the Liao, Hai and Yellow rivers draining into the Bohai Gulf; the Huai River draining into the Yellow Sea; the Yangtze River draining into the East China Sea; and the Pearl River draining into the South China Sea. River drainage areas are delineated using the global drainage direction map (DDM-30, 30 arc minute resolution) (Döll and Lehner, 2002). The country boundary is from (RESDC, 2013).

\subsubsection{The MARINA 2.0 model}

We used the MARINA model version 2.0 (Wang et al., Under Review) to explore future nutrient pollution in rivers and coastal waters of China. The MARINA 2.0 model was developed to quantify river export of TDN and TDP in four forms by rivers at the subbasin scale from different sources (Wang et al., Under Review). The four nutrient forms are: dissolved inorganic (DIN) and organic (DON) N, and dissolved inorganic (DIP) and organic (DOP) P. TDN is the sum of DIN and DON, and TDP is the sum of DIP and DOP.

MARINA 2.0 quantifies river export of TDN and TDP as a function of $\mathrm{N}$ and $\mathrm{P}$ inputs to surface waters (rivers) from diffuse and point sources, and retentions of $\mathrm{N}$ and $\mathrm{P}$ in rivers based on the overall equation below (Strokal et al., 2016a; Wang et al., Under Review):

$M_{F . y . j}=\left(R_{S d i f}\right.$.y.j.j + RSpntF.y.j $)$ FEriv.F.outlet.j $\cdot$ FEriv.F.mouth.j 
Where MF.y.j is river export of $\mathrm{N}$ and $\mathrm{P}$ in form F (DIN, DON, DIP, DOP) by source y from sub-basin $\mathrm{j}\left(\mathrm{kg}\right.$ year-1). ${ }^{-1}$ Sdiff.y.j is $\mathrm{N}$ and $\mathrm{P}$ inputs in form $\mathrm{F}$ to rivers (surface waters) from diffuse sources $y$ in sub-basin $\mathrm{j}\left(\mathrm{kg}_{\mathrm{g}} \mathrm{year}^{-1}\right)$. RSpntF.y.j is $\mathrm{N}$ and $\mathrm{P}$ inputs in form $\mathrm{F}$ to rivers from point sources $\mathrm{y}$ in sub-basin $\mathrm{j}\left(\mathrm{kg}\right.$ year $\left.{ }^{-1}\right)$. FEriv.F.outlet.j is the fraction of $\mathrm{N}$ and $\mathrm{P}$ in form $\mathrm{F}$ exported to the outlet of sub-basin $\mathrm{j}(0-1)$. FEriv.F.mouth.j is the fraction of $\mathrm{N}$ and $\mathrm{P}$ in form $\mathrm{F}$ exported from the outlet of sub-basin $\mathrm{j}$ to the river mouth (0-1). The detailed equations to quantify RSdiff.y.j, RSpntF.y.j, FEriv.F.outlet.j and FEriv.F.mouth.j are available in the SI of Wang et al. (Under Review).

\subsubsection{Indicators for SDGs 6 and 14}

Two indicators were calculated from the MARINA 2.0 results to assess whether SDGs 6 and 14 are met. We used water quality standards for $\mathrm{N}$ and $\mathrm{P}$ concentrations as the indicator for SDG 6, and the Indicator for Coastal Eutrophication Potential (ICEP) for SDG 14. Below we describe how these indicators were chosen and calculated.

The goal of SDG 6 is to "ensure availability and sustainable management of water and sanitation for all" (UN, 2015). One important indicator for assessing SDG 6 is "6.3.2 proportion of bodies of water with good ambient water quality", according to the global indicator list from the UN (IAEG, 2016). The Chinese government aims to meet SDG 6 by implementing the "Water Pollution Prevention and Control Action Plan" (SCPRC, 2015), which defines the standard for good ambient water quality in China. This standard is based on the Chinese "Environmental Quality Standard for Surface Water" for "thirdgrade" (grade-III) water quality (MEP, 2002). For "grade III" water in rivers, the concentration of ammonia ( $\mathrm{NH}_{3}$ ) may not exceed $1.0 \mathrm{mg}-\mathrm{N} / \mathrm{L}$, and total phosphorus (TP) may not exceed $0.2 \mathrm{mg}-\mathrm{P} / \mathrm{L}$. The MARINA 2.0 model quantifies DIN (includes ammonia $\left(\mathrm{NH}_{3}\right)$, nitrate $\left(\mathrm{NO}_{3}^{-}\right)$and nitrite $\left(\mathrm{NO}_{2}^{-}\right)$) and TDP, but not $\mathrm{NH}_{3}$ and TP. Therefore, we calculated $\mathrm{N}$ and $\mathrm{P}$ concentrations at the outlets of sub-basins using modeled DIN and TDP loads and river discharges at the outlets. We compared the calculated concentrations of DIN and TDP with the water quality standards for "grade-III" water, and discussed whether our scenarios contribute to the achievement of SDG 6.

The goal of SDG 14 is to "conserve and sustainably use the oceans, seas and marine resources for sustainable development" (UN, 2015). The global indicator list from the UN suggests "14.1.1 Index of coastal eutrophication" as an indicator for this SDG (IAEG, 2016). Therefore, we took ICEP as an indicator for assessing SDG 14 in our study. ICEP indicates 
the potential for new production of harmful (non-siliceous) algae in coastal waters. This indicator is calculated by comparing the N, P and Si loads and the Redfield molar ratios (C:N:P:Si ratios 106:16:1:20) (see Garnier et al. (2010) for the detailed approach to quantify ICEP). Positive ICEP values indicate relatively high potentials for harmful algal blooms when rivers deliver excess $\mathrm{N}$ or P over silica (Si) to the sea. Negative ICEP values indicate relatively low potentials for harmful algal blooms. We calculated the ICEP values for the six Chinese rivers using modeled river export of TDN and TDP by MARINA 2.0. Based on the results we discussed whether our scenarios contribute to the achievement of SDG 14.

\subsubsection{Scenarios}

We developed six scenarios and assessed their impacts on future nutrient pollution in rivers and coastal waters in China. The storylines of these scenarios combine improved nutrient management in agriculture and sewage, efficiency and healthy food production, and climate mitigation. The main assumptions for the scenarios are also summarized in Table 7.1.

The baseline SSP5-RCP8.5 scenario assumes relatively low population growth, fast economic growth, high fossil-fuel consumption, high international trade, increasing productivity in agriculture and environmental policies for local issues (O'Neill et al., 2017; Wang et al., 2017; Wang et al., Under Review). As a result, in 2050 sewage systems will be slightly improved compared to today. Not all wastewater will be connected to sewage systems, especially in the rural areas where only $10 \%$ of the wastewater will be collected (Table 7.1). Nutrient removal during treatment will remain low or moderate at around $12-47 \%$ for $\mathrm{N}$, and $44-75 \%$ for $\mathrm{P}$ in rural and urban areas (Table 7.1). Crops will be produced with fewer resources (e.g., nutrient, land and water) because of the increased productivity. Animal production will be intensive and industrialized to meet the increasing preference for meat-rich diets. Improved manure management is implemented to reduce emissions of ammonia $\left(\mathrm{NH}_{3}\right)$ and nitrous oxide $\left(\mathrm{N}_{2} \mathrm{O}\right)$ during manure storage and housing. About $15-41 \%$ of crop residues and $70 \%$ of animal manure will be recycled in agriculture (Table 7.1). The rest is lost to the environment. The import of food for consumption will be $17 \%$ higher in 2050 than in 2012 (Table 7.1). The greenhouse gas emissions by China, and in other countries, will be high as the result of high fossil-fuel consumption. 
The SE (improved sewage treatment) scenario builds on SSP5-RCP8.5 and assumes further improved sewage systems in 2050. According to current Chinese policies, wastewater connected to sewage systems will reach to $70-95 \%$ in urban areas, and $60 \%$ in one-third counties of China including both rural and urban areas (Table 7.1). We, therefore, assume in this scenario that in 2050 all wastewater will be connected to centralized (in urban areas) or decentralized (in rural areas) sewage systems, following Strokal et al. (2017). Nutrient removal during treatment is assumed to reach $80 \%$ for $\mathrm{N}$, and $90 \%$ for P by adopting the best treatment technologies (Strokal et al., 2017; Van Puijenbroek et al., 2019) (Table 7.1).

The AG (improved nutrient use efficiencies in agriculture) scenario builds on SSP5RCP8.5 and assumes further improved nutrient use efficiencies in agriculture in 2050. In this scenario, crops will be fertilized according to their needs for nutrients base on a balanced fertilization approach (Ma et al., 2013a; Wang et al., 2018a). As a result, the use of synthetic fertilizers will be largely reduced compared to the baseline, without yield losses. Recycling of straw residues on cropland will increase to $80 \%$ in order to reduce air pollution from straw burning (Table 7.1). Animal production will be more efficient by using improved animal feeding and genetically modified animals that take up nutrients more efficiently (Oenema et al., 2009; Wang, 2005a; Wang, 2005b). In AG, N and P excretion are thus $12 \%$ lower than in the baseline SSP5-RCP8.5 (Table 7.1). Improved manure management is incorporated to reduce emissions of $\mathrm{NH}_{3}$ and $\mathrm{N}_{2} \mathrm{O}$ during manure storage (Hou et al., 2017; Hou et al., 2015; Zheng et al., 2014). In AG, direct discharge of manure will be restricted by policies, thus all manure is assumed to be treated and recycled on cropland.

The AG+SE (combination of AG and SE) scenario combines the storylines of SE and AG. It assumes improved sewage systems and improved nutrient use efficiencies in agriculture.

The AG+SE+SFC (sustainable food consumption in addition to AG+SE) scenario builds on AG+SE and assumes healthier diets and less food waste in 2050. In this scenario, the society will follow the Chinese food dietary guidelines (CDGs) (Chinese Nutrition Society, 2007), which recommends consuming less meat but more milk, eggs, vegetables, and fruits. Food waste will be reduced by $20 \%$ through responsible consumptions, improved food processing, and storage facilities (Ma et al., 2019). The reduction in meat 
consumption and food waste will result in a reduction of $20 \%$ in requirements for crop and animal production. China may still remain as a large importer of soybean due to limited land resources and increasing food demand (FAO, 2019). For soybeans, we assumed that around $80 \%$ of the soybean consumption in 2050 will be imported from abroad following the assumption in Ma et al. (2019). In addition to the above assumptions, this scenario assumes further improved management of animal manure. According to the $\mathrm{AG}+\mathrm{SE}$ scenario, many river basins has too few land area to recycling all manure produced in the basins. Therefore $\mathrm{AG}+\mathrm{SE}+\mathrm{SFC}$ assumes that the excessive manure will be either treated or exported to other regions to be recycled in China. Last but not least, the atmospheric $\mathrm{N}$ deposition will be reduced by $50 \%$ relative to SSP5-RCP8.5 by mitigating $\mathrm{NH}_{3}$ and $\mathrm{N}_{2} \mathrm{O}$ emissions in the agriculture and non-agriculture sectors (e.g., control of $\mathrm{NH}_{3}$ and $\mathrm{N}_{2} \mathrm{O}$ emissions from industries).

The $\mathbf{A G}+\mathbf{S E}+\mathbf{S F C}+\mathbf{C L I}$ (climate mitigation in addition to $\mathbf{A G}+\mathbf{S E}+\mathbf{S F C}$ ) scenario builds on $\mathrm{AG}+\mathrm{SE}+\mathrm{SFC}$ and assumes a global effort in climate mitigation in 2050. In the MARINA 2.0 model (Wang et al., Under Review), The baseline SSP5-RCP8.5 scenario assumes high greenhouse gas (GHG) concentrations under higher fossil-fuel consumption. This will lead to a high level of climate change and thus affect hydrology (e.g, river discharge). $\mathrm{AG}+\mathrm{SE}+\mathrm{SFC}+\mathrm{CLI}$ assumes that GHG emissions will be reduced to the level of RCP2.6 in 2050. This implies efforts in reducing GHG emissions by countries worldwide to achieve the Paris temperature targets (UNFCCC, 2015). The lower GHG emissions in the future may result in less increases in precipitation and river discharge than in the baseline, and thus less decreases in in-river retention of nutrients. River export of nutrients may thus be reduced by climate mitigation in this scenario, compared to the baseline. 


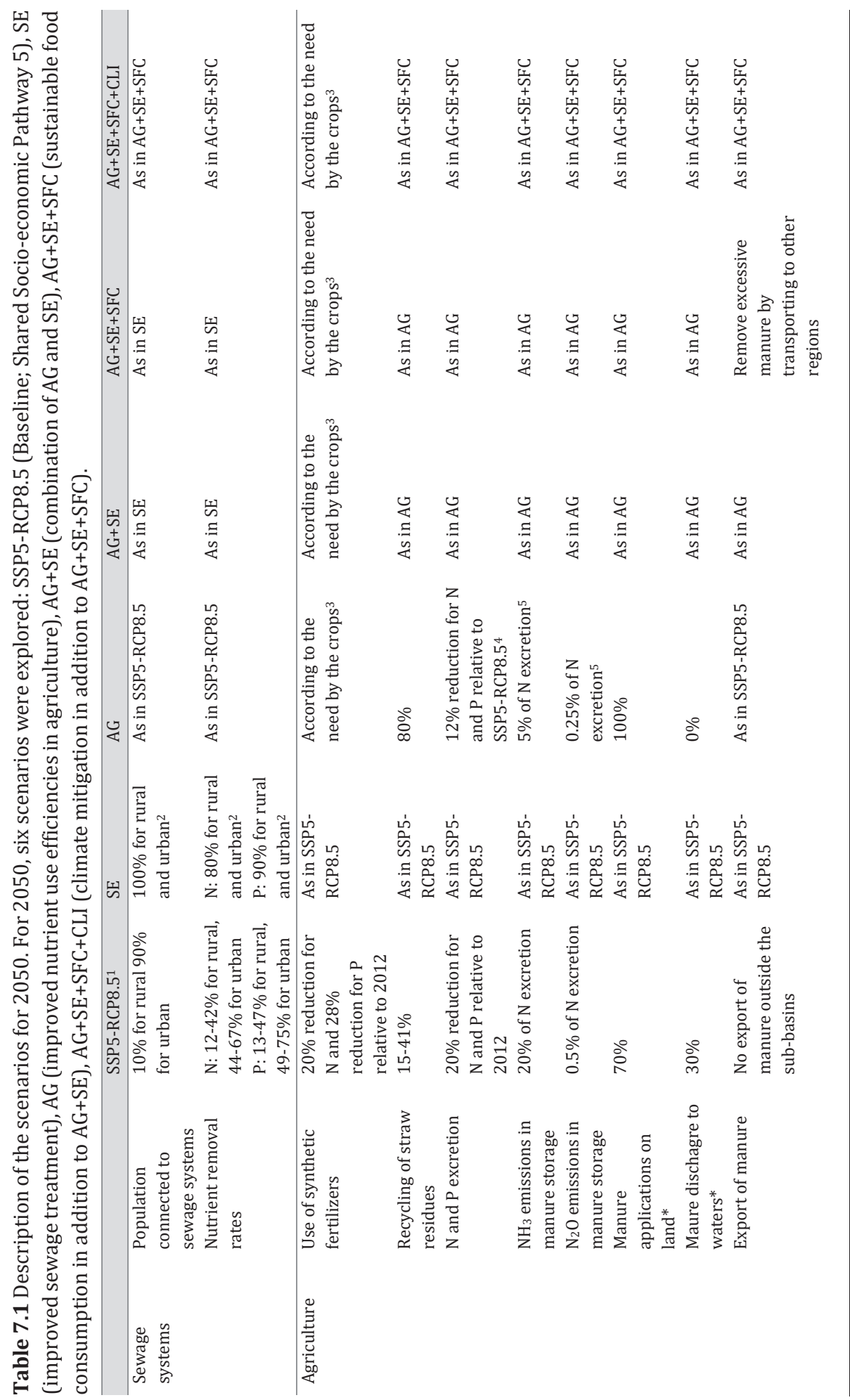




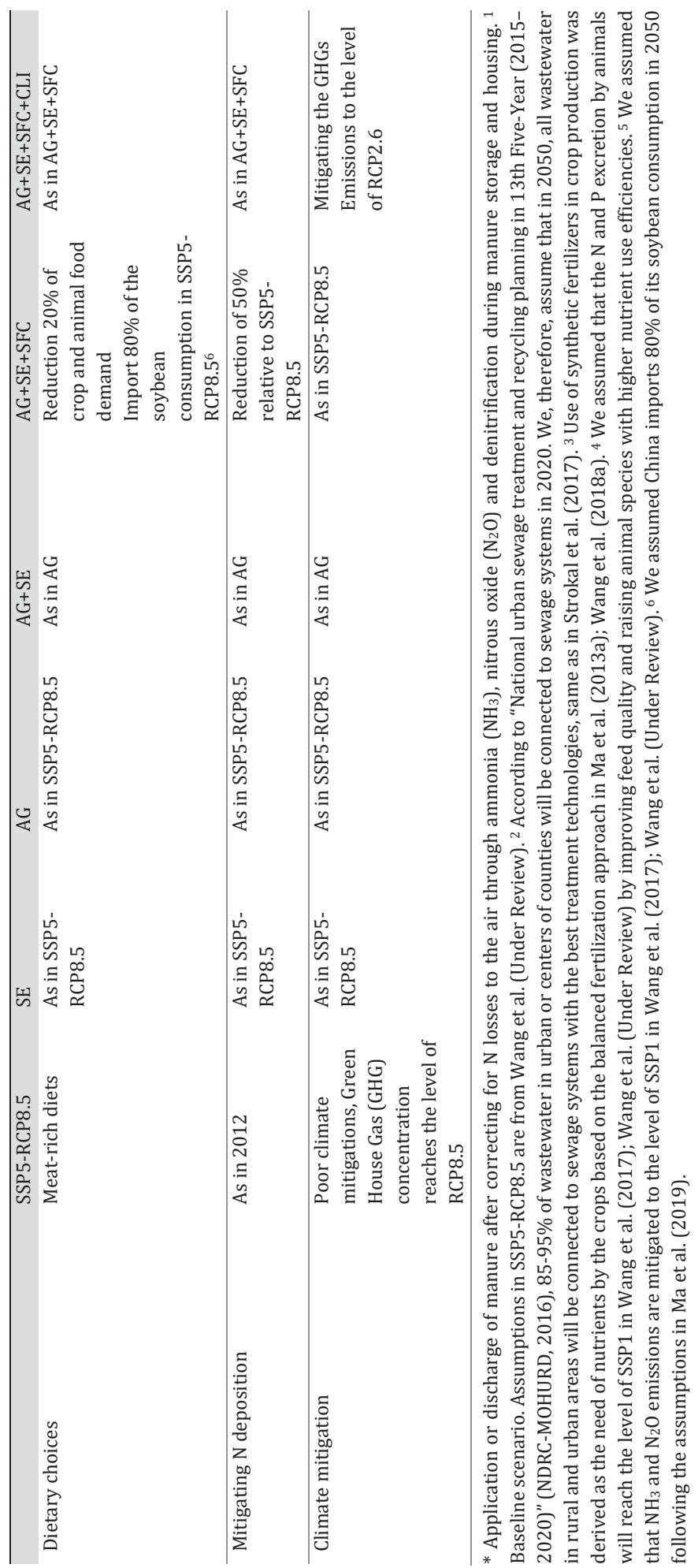




\subsection{Reducing nutrient pollution in rivers and coastal waters}

2012. Nutrient pollution levels in the six large rivers and coastal waters were relatively high in 2012. We calculate 18,553 kton of TDN and 2143 kton of TDP inputs to rivers (Figure 7.2). These nutrients are further transported by the rivers, leading to export of 4341 kton of TDN and 644 kton of TDP to the Chinese seas (Figure 7.2). More than half of these TDN and TDP are exported to the East China Sea (Figure 7.3). The rest is exported to the Bohai Gulf (15\% of TDN, 23\% of TDP), Yellow Sea (6\% of TDN, $10 \%$ of TDP) and South China Sea (25\% of TDP, 14\% of TDP). River export of TDN and TDP in 2012 mainly origins from human activities on land including direct discharge of animal manure, use of synthetic fertilizers, and discharge of (treated or untreated) human waste (Figure 7.2, Figures B.1-B.4 in Appendix VI).

Baseline scenario SSP5-RCP8.5. In SSP5-RCP8.5, TDN input to rivers and river export of TDN to seas in 2050 are projected to remain as high as in 2012. However, TDP input to rivers in 2050 is 38\% lower than that in 2012. As a result, river export of TDP is $42 \%$ lower compared to 2012. The decrease in P pollution can be largely explained by the improved manure management in SSP5-RCP8.5. In this scenario, recycling of animal manure (the amount available for application after losses during manure storage) is assumed to improve from 46\% to 70\% between 2012 and 2050 (Wang et al., 2017) (Table 7.1). This reduces largely the direct discharge of manure to rivers in 2050. However, TDN input to rivers from direct discharge of manure to rivers remains high in SSP5-RCP8.5. This is the net effect of increased manure recycling, and reduced ammonia $\left(\mathrm{NH}_{3}\right)$ emissions during manure storage and housing (increased $\mathrm{N}$ in manure available for application) in SSP5-RCP8.5 (Table 7.1). Human activities on land (e.g., direct discharge of animal manure, use of synthetic fertilizers, and discharge of human waste) remain the dominant sources of river export of TDN and TDP to Chinese seas (Figure 7.2, Figures B.1B.4 in Appendix VI).

Future nutrient pollution in rivers and coastal waters may be lower in 2050, but the level varies among the alternative scenarios (Figures 7.2 and 7.3). AG+SE+SFC+CLI is found to be the most effective scenario to improve water quality. Below we discuss the result of each scenario for 2050 .

Alternative scenario SE. In this scenario, all wastewater from rural and urban areas is connected to sewage systems with relatively high nutrient removal efficiencies. Thus in 
2050, TDN and TDP inputs to rivers from treated and untreated human waste is calculated to reduce by $54 \%$ and $79 \%$, respectively, compared to SSP5-RCP8.5. River export of nutrients is $9 \%$ lower for TDN, and 25\% lower for TDP in SE than in the baseline SSP5RCP8.5.

Alternative scenario AG. Improved nutrient management in agriculture in AG is projected to be more effective than SE to reduce future nutrient pollution in rivers and coastal water. We quantified 12,091 kton of TDN and 551 kton of TDP inputs to rivers in 2050 in AG. River export of TDN is 39\% lower than in the baseline SSP5-RCP8.5, and of TDP 63\% lower. Nutrient pollution in rivers and coastal waters are much reduced by restricting direct discharge of animal manure, recycling of manure on land, and reducing over-fertilization in crop production.

Alternative scenario AG+SE. This scenario combines the improved nutrient management of $\mathrm{SE}$ and $\mathrm{AG}$, and thus combines their effectiveness in reducing nutrient pollution in rivers and coastal waters in 2050. Nutrient inputs to rivers in AG+SE are projected to be $42 \%$ lower for TDN, and $88 \%$ lower for TDP compared to the baseline SSP5-RCP8.5. River export of TDN is 48\% lower, and of TDP is $89 \%$ lower than in SSP5RCP8.5. $\mathrm{N}$ and $\mathrm{P}$ inputs to the sub-basins from agriculture and human waste in this scenario.

Alternative scenario $\mathbf{A G}+\mathbf{S E}+\mathbf{S F C}$. We calculate further reduction in nutrient pollution in rivers and coastal waters in this scenario compared to $\mathrm{AG}+\mathrm{SE}$. This scenario builds on $\mathrm{AG}+\mathrm{SE}$ and assumes healthy and efficient food consumption, treatment of excessive manure, and mitigation of $\mathrm{N}$ deposition. Nutrient use in crop and animal production is lower than in $\mathrm{AG}+\mathrm{SE}$ because of reduced consumption of meat, less food waste and import of soybeans. We calculate $7870 \mathrm{kton}$ of TDN, and $46 \mathrm{kton}$ of TDP inputs to rivers in 2050 in AG+SE+SFC. These nutrient inputs to rivers result in export of $1714 \mathrm{kton}$ of TDN and 39 kton of TDP to seas. This amount is $60 \%$ and $90 \%$ lower for TDN and TDP, respectively, compared to the baseline SSP5-RCP8.5.

Alternative scenario $\mathbf{A G + S E + S F C + C L I}$. This scenario is found to be the most effective to reduce nutrient pollution in rivers and coastal waters in China. In addition to the improve nutrient management in $\mathrm{AG}+\mathrm{SE}+\mathrm{SFC}$, this scenario assumes optimistic climate mitigation by countries in the future. The climate-induced decreases in water runoff and discharge are projected to reduce TDN and TDP inputs to rivers by $15 \%$ and $9 \%$ for TDN and TDP, 
respectively, compared to $\mathrm{AG}+\mathrm{SE}+\mathrm{SFC}$. As a result, in 2050, river export of $\mathrm{TDN}$ is $68 \%$ lower, and of TDP 91\% lower in AG+SE+SEF+CLI than in SSP5-RCP8.5. Combing the best nutrient management in all other alternative scenarios, $\mathrm{N}$ and $\mathrm{P}$ inputs to rivers from human activities (i.e., agriculture and human waste) are much reduced in this scenario (Figure 7.2). Thus other sources (i.e., biological $\mathrm{N}_{2}$ fixation, atmospheric $\mathrm{N}$ deposition, $\mathrm{P}$ weathering, and leaching of organic matters) may become important sources of nutrient pollution in rivers and coastal waters in 2050 (Figure 7.2, Figures B.1-B.4 in Appendix VI).

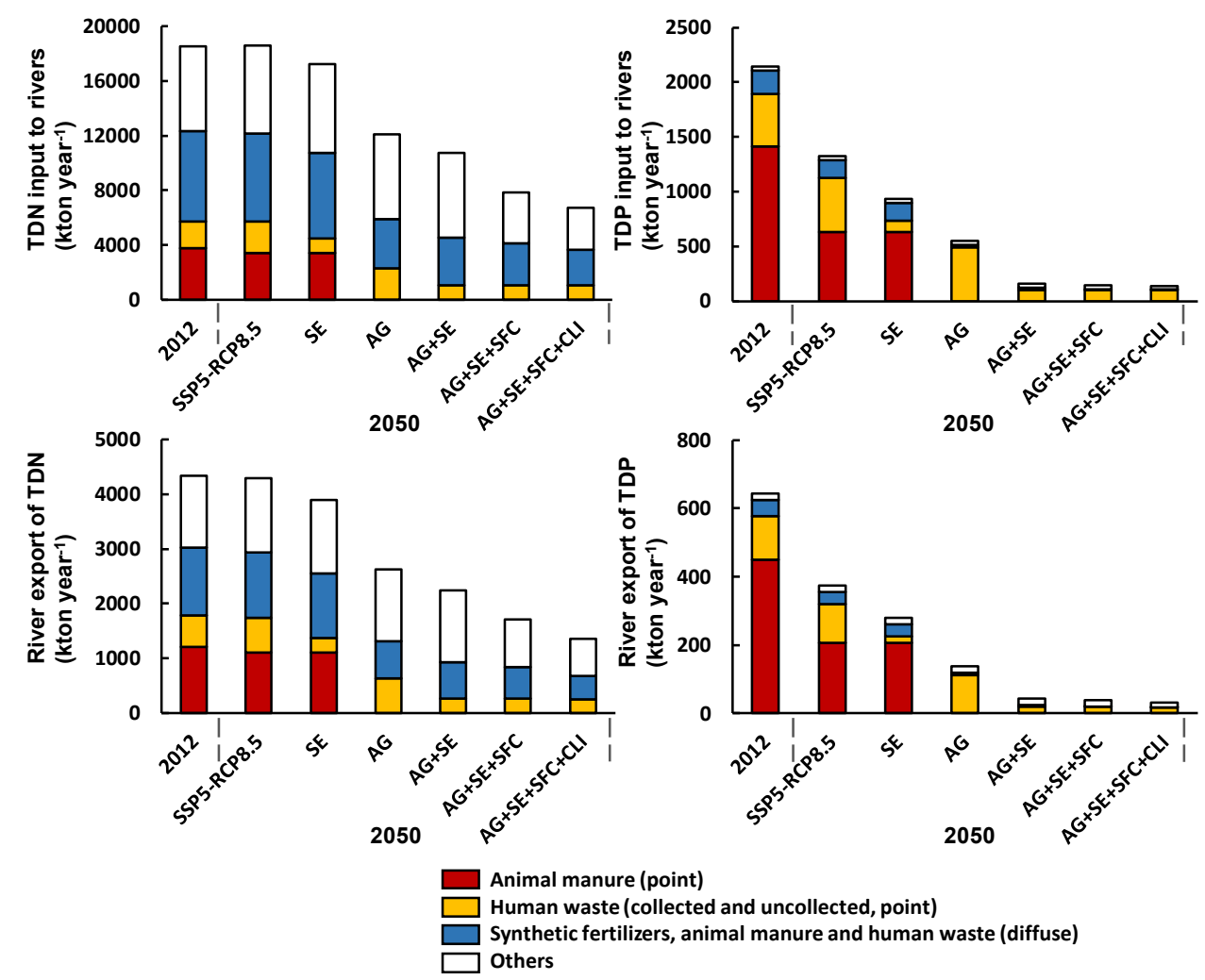

Figure 7.2 Total dissolved nitrogen (TDN) and phosphorus (TDP) inputs to rivers, and river export of TDN and TD) by source from six large rivers to Chinese seas in 2012 and 2050 (kton year-1 $^{-1}$. The six large rivers are: Liao, Hai, Yellow, Huai, Yangtze and Pearl rivers. For 2050, six scenarios were explored: SSP5-RCP8.5 (Shared Socio-economic Pathway 5-Representative Concentration Pathway 8.5), SE (improved sewage treatment), AG (improved nutrient use efficiencies in agriculture), AG+SE (combination of AG and SE), AG+SE+SFC (sustainable food consumption in addition to $\mathrm{AG}+\mathrm{SE}$ ), $\mathrm{AG}+\mathrm{SE}+\mathrm{SFC}+\mathrm{CLI}$ (climate mitigation in addition to $\mathrm{AG}+\mathrm{SE}+\mathrm{SFC}$ ). Others include biological $\mathrm{N}_{2}$ fixation, atmospheric $\mathrm{N}$ deposition and leaching of organic matter for TDN, and include $\mathrm{P}$ weathering and leaching of organic matter for TDP. 


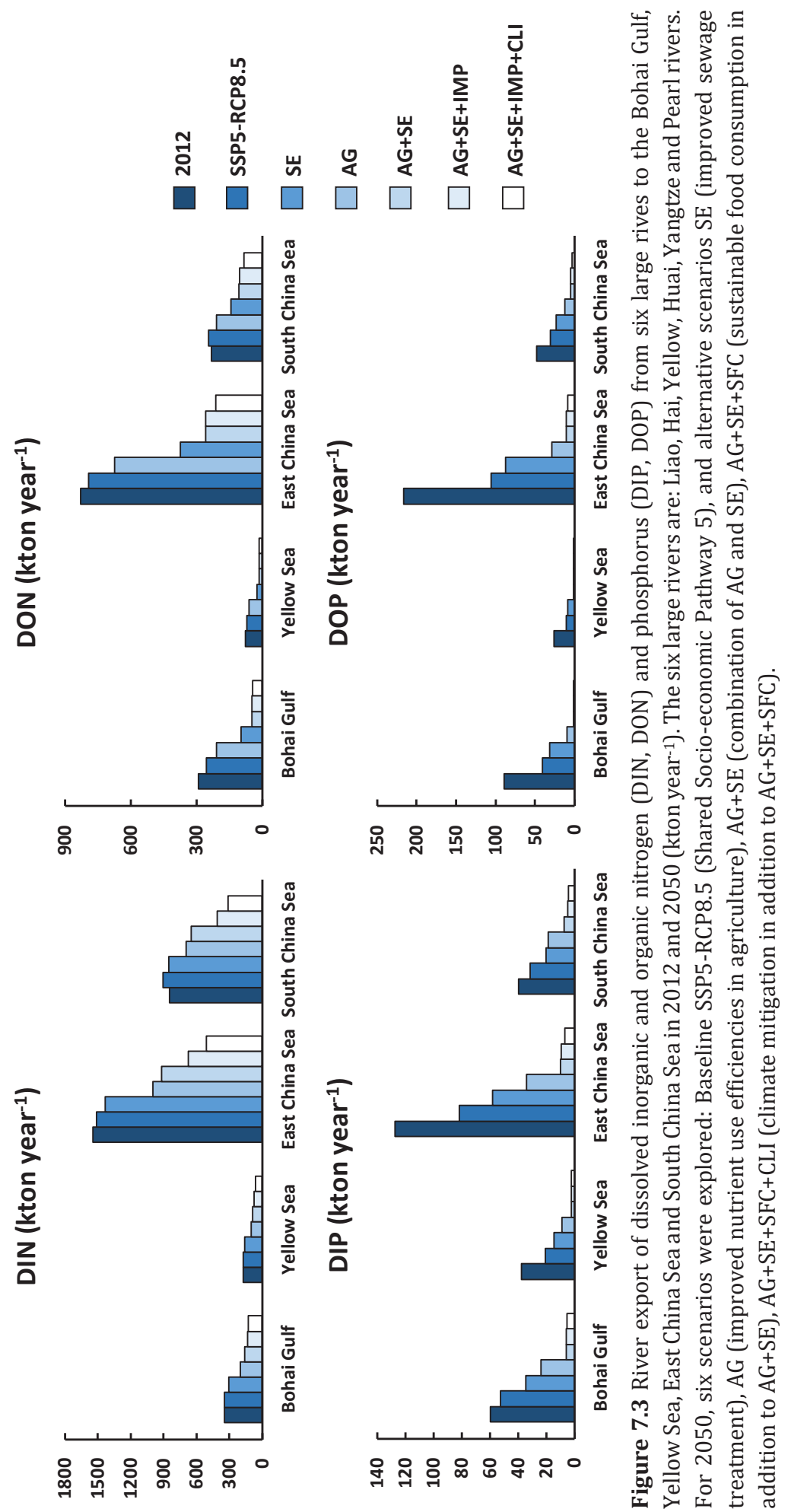




\subsection{Achieving the SDGs in China}

Our scenario analysis shows that improved nutrient management in agriculture and sewage systems, healthy and efficient food consumption and climate mitigation may contribute to meeting SDGs 6 and 14, and several other SDGs in the future. Below we discuss the contributions to the SDGs.

\subsubsection{SDG 6 and 14}

SDG 6. We calculated that water quality at 23 out of the 26 sub-basin outlets is worse than "grade-III" water for either DIN (1.0 mg/L) or TDP (0.2 mg/L) in 2012 (Figures 7.4 and 7.5). The levels of nutrient pollution in rivers remain high in 2050 in the baseline SSPRCP8.5 scenario, as a result of high nutrient inputs to rivers (Figures 7.4 and 7.5). Results show that the alternative scenarios may be effective to meet SDG 6 by reducing nutrient pollution in rivers in the future (Figures 7.4 and 7.5). The effectiveness varies among scenarios and sub-basins, as we discussed in Section 7.3.1. In the most effective scenario $\mathrm{AG}+\mathrm{SE}+\mathrm{SFC}+\mathrm{CLI}$, river water quality may be improved considerably compared to SSP5RCP8.5 (Figures 7.4 and 7.5). We estimated water quality at all sub-basin outlets better than "grade III" base on TDP concentrations. Only outlets of 5 sub-basins may have DIN concentrations meeting the standard for 'grade III'. However, in this study, we compared the DIN concentrations with the standard for $\mathrm{NH}_{3}$. Therefore in those outlets where other inorganic nitrogen forms (e.g., $\mathrm{NO}_{2}^{-}, \mathrm{NO}_{3}^{-}$) dominant in water, the concentrations of $\mathrm{NH}_{3}$ may meet the standard for "grade III" water. Nevertheless, the results show that one of the challenges to meet SDG 6 for China is to reduce $\mathrm{N}$ pollution in rivers. This is in line with the study of Yu et al. (2019) who concluded that reducing $\mathrm{N}$ pollution for a clean water environment in China is challenging because of the additional sources of $\mathrm{N}$ losses (e.g., atmospheric $\mathrm{N}$ deposition, biological $\mathrm{N}_{2}$ fixation).

SDG 14. We calculated high potentials of coastal eutrophication at the river mouth of all six rivers in 2012 and 2050 for SSP5-RCP8.5, indicated by positive ICEP values (Figure

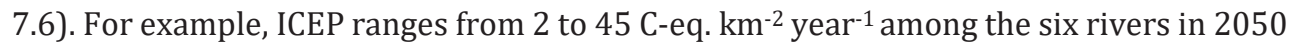
for SSP5-RCP8.5. Improved management in the alternative scenarios may contribute to meeting SDG 14 by reducing the potential for coastal eutrophication: we calculated lower ICEP values in the alternative scenarios compared to SSP5-RCP8.5 (Figure 7.6). In the $\mathrm{AG}+\mathrm{SE}+\mathrm{SFC}+\mathrm{CLI}$ scenario, negative ICEP values are projected for Huai, Yangtze, and Pearl rivers, indicating low potentials for coastal eutrophication. However, the Liao, Hai and 
Yellow rivers draining into the Bohai Gulf may still have high potentials for coastal eutrophication, indicated by the positive ICEP values.

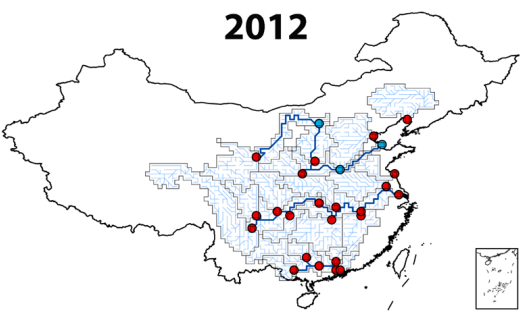

\section{DIN}
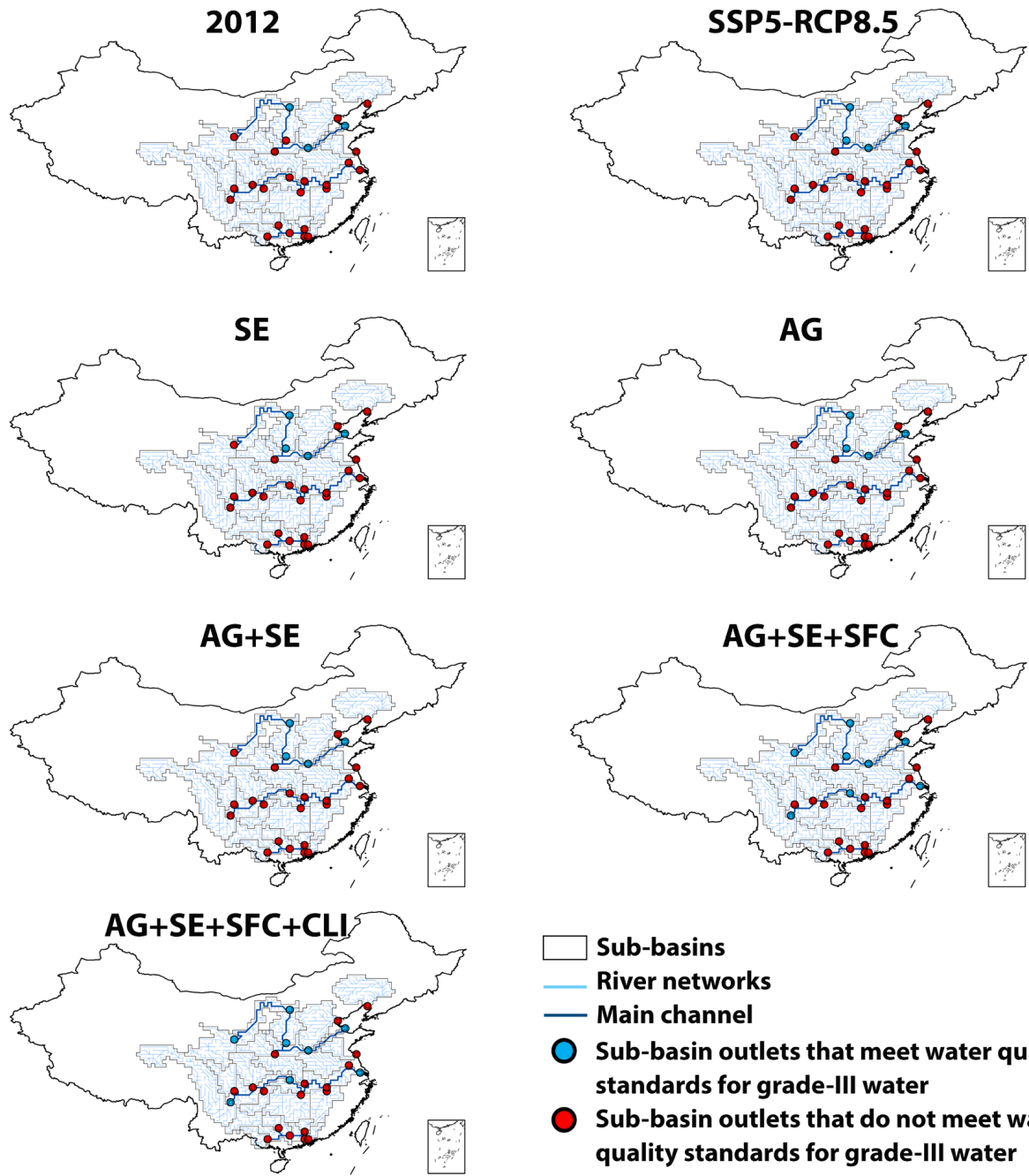

Sub-basins

River networks

Main channel

Sub-basin outlets that meet water quality standards for grade-III water

Sub-basin outlets that do not meet water quality standards for grade-III water

Figure 7.4 Concentrations of dissolved organic nitrogen (DIN) at the outlets of sub-basins versus the water quality standards for "grade-III" water in China (concentrations of ammonia- $\mathrm{NH}_{3}<1$ $\mathrm{mg} \mathrm{N} / \mathrm{L}$ ) in 2012 and 2050. This comparison is to assess if SDG 6 - "Ensure access to water and sanitation for all" is met at the sub-basin outlets. The water quality standards for "grade-III" water are from the Chinese "Environmental Quality Standard for Surface Water" (MEP, 2002). For 2050, six scenarios were explored: Baseline SSP5-RCP8.5 (Shared Socio-economic Pathway 5), and alternative scenarios SE (improved sewage treatment), AG (improved nutrient use efficiencies in agriculture), $\mathrm{AG}+\mathrm{SE}$ (combination of $\mathrm{AG}$ and $\mathrm{SE}$ ), $\mathrm{AG}+\mathrm{SE}+\mathrm{SFC}$ (sustainable food consumption in addition to $\mathrm{AG}+\mathrm{SE}$ ), $\mathrm{AG}+\mathrm{SE}+\mathrm{SFC}+\mathrm{CLI}$ (climate mitigation in addition to $\mathrm{AG}+\mathrm{SE}+\mathrm{SFC}$ ). 


\section{TDP}
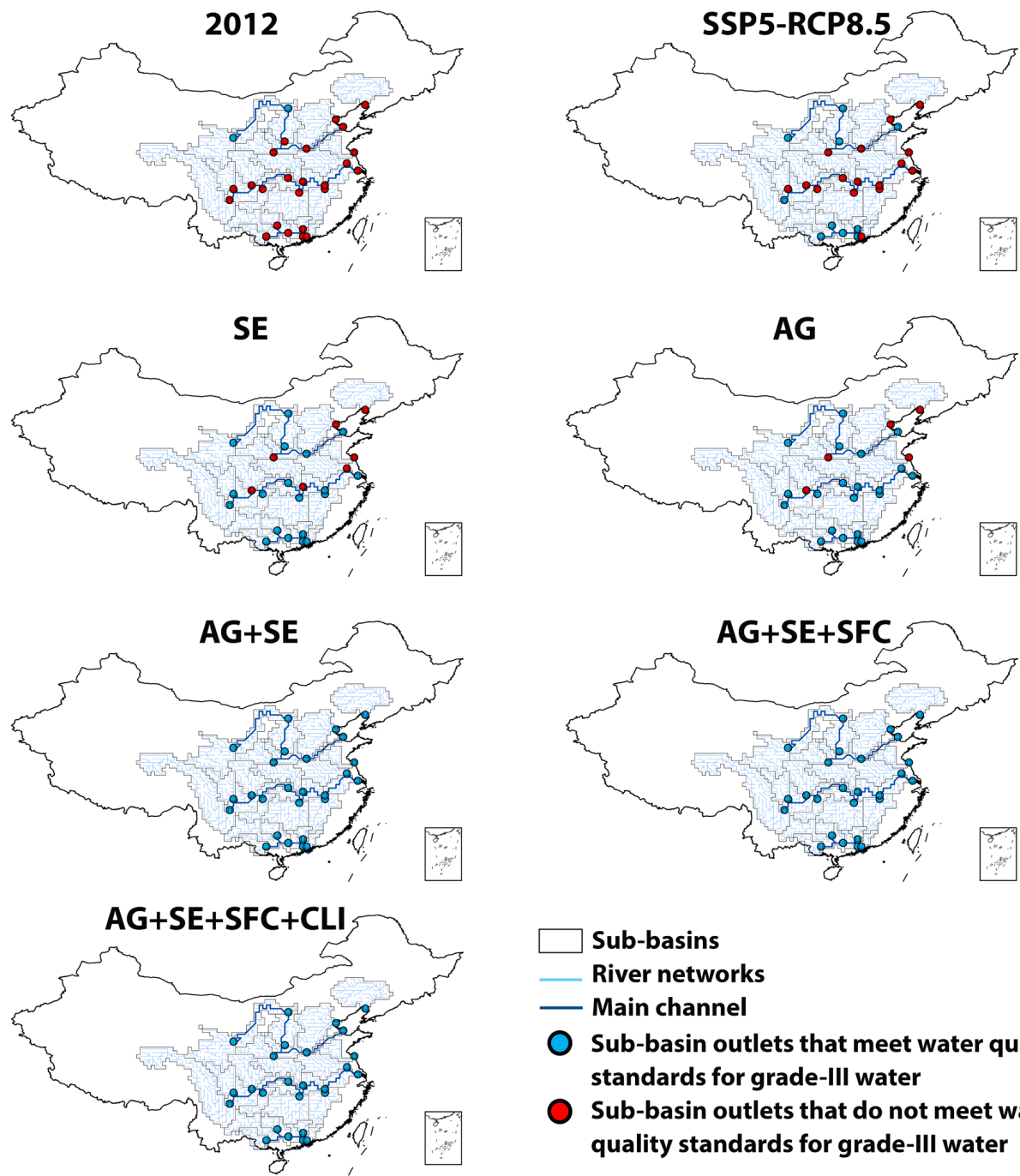

Sub-basins

River networks

Main channel

Sub-basin outlets that meet water quality standards for grade-III water

Sub-basin outlets that do not meet water quality standards for grade-III water

Figure 7.5 Concentrations of total dissolved phosphorus (TDP) at the outlets of sub-basins versus the water quality standards for "grade-III" water in China (concentrations of total phosphorus < $0.2 \mathrm{mg} \mathrm{N} / \mathrm{L}$ ) in 2012 and 2050. This comparison is to assess if SDG 6 - "Ensure access to water and sanitation for all" is met at the sub-basin outlets. The water quality standards for "grade-III" water are from the Chinese "Environmental Quality Standard for Surface Water" (MEP, 2002). For 2050, six scenarios were explored: Baseline SSP5-RCP8.5 (Shared Socio-economic Pathway 5), and alternative scenarios SE (improved sewage treatment), AG (improved nutrient use efficiencies in agriculture), $\mathrm{AG}+\mathrm{SE}$ (combination of $\mathrm{AG}$ and $\mathrm{SE}$ ), $\mathrm{AG}+\mathrm{SE}+\mathrm{SFC}$ (sustainable food consumption in addition to $\mathrm{AG}+\mathrm{SE}$ ), $\mathrm{AG}+\mathrm{SE}+\mathrm{SFC}+\mathrm{CLI}$ (climate mitigation in addition to $\mathrm{AG}+\mathrm{SE}+\mathrm{SFC}$ ). 


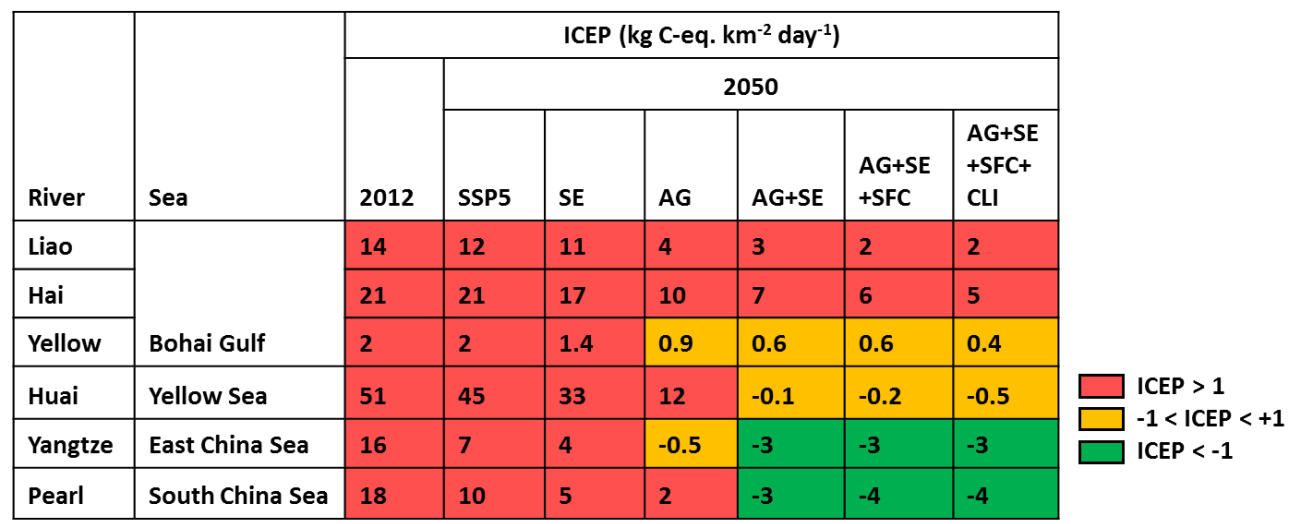

Figure 7.6 Indicator for Coastal Eutrophication Potential (ICEP) for the six large rivers in China

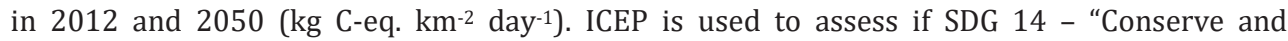
sustainably use the oceans, seas and marine resources" is met at the river mouths. A positive ICEP value indicates that river export of nutrients is in excess over silica, and thus indicates high potentials for coastal eutrophication. A negative ICEP value indicates low potentials for coastal eutrophication. Following the approach of (Garnier et al., 2010), we calculated ICEPs based on our modeled river export of dissolved nitrogen and phosphorus, and river export of Si from the Global NEWS-2 model (Seitzinger et al., 2010). For 2050, six scenarios were explored: Baseline SSP5RCP8.5 (a combination of Shared Socio-economic Pathway 5 and Representative Concertation Pathway 8.5), and alternative scenarios SE (improved sewage treatment), AG (improved nutrient use efficiencies in agriculture), AG+SE (combination of AG and SE), AG+SE+SFC (sustainable food consumption in addition to $\mathrm{AG}+\mathrm{SE}$ ), $\mathrm{AG}+\mathrm{SE}+\mathrm{SFC}+\mathrm{CLI}$ (climate mitigation in addition to $\mathrm{AG}+\mathrm{SE}+\mathrm{SFC}$.

\subsubsection{Other SDGs.}

From the above is clear that our future scenarios contribute to meeting SDGs 6 and 14 . Here we discuss the implications of our scenarios for some other SDGs (Figure 7.7, Table 7.1). There may be several effects. For instance, increased nutrient use efficiencies in agriculture as assumed in the AG scenario may contribute to meeting SDG 2 - "zero hunger". The improved fertilizer and manure management that mitigates $\mathrm{NH}_{3}$ and $\mathrm{N}_{2} \mathrm{O}$ emissions may reduce atmospheric $\mathrm{N}$ deposition. This will likely contribute to reducing threats to biodiversity in the ecosystems, and thus contribute to SDG 15 - "Sustainably manage forests, combat desertification, halt and reverse land degradation, halt biodiversity loss". In SE, all wastewater from rural and urban areas is assumed to be connected to the sewage systems with improved nutrient treatment efficiency. This will likely contribute to meeting SDG 11 - "Make cities inclusive, safe, resilient and sustainable". The diet change towards lower meat consumption, and reducing food waste in $\mathrm{AG}+\mathrm{SE}+\mathrm{SFC}$ would result in a reduction of the requirements for crop and animal 
production. This, therefore, may contribute to SDG 12 - "Ensure sustainable consumption and production patterns". Last but not least, the climate mitigation efforts by countries in the AG+SE+SFC+CLI scenario would contribute to SDG 13 - "Take urgent action to combat climate change and its impacts".

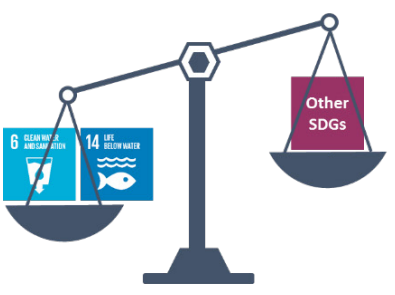

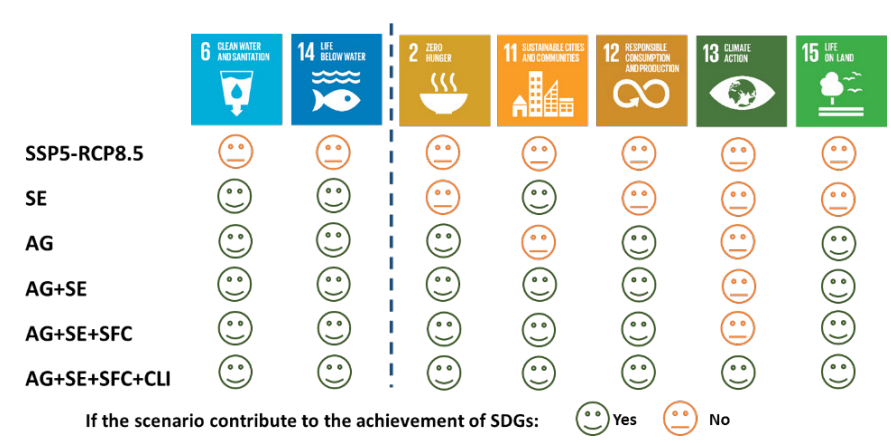

Figure 7.7 Contribution of the six scenarios to the achievement of seven relevant SDGs. 'Yes' indicates the scenario may contribute to meeting the SDGs. 'No' indicates that the scenario may not contribute to meeting the SDGs. This assessment is based on the modeled nutrient pollution in rivers and coastal waters (Figures 7.2 and 7.3), and the assumptions on nutrient management in agriculture and sewage, food consumption and climate mitigation in this study (Table 7.1). A detailed explanation of how the SDGs are assessed for the six scenarios is available in Table B.1 in Appendix VI.

\subsection{Discussion}

We explored future scenarios to reduce nutrient pollution in rivers and coastal waters of China. We analyzed how these future scenarios will contribute to SDGs 6 and 14 from the perspective of nutrient pollution. Results show that combing improved nutrient management in agriculture and sewage systems, healthy and efficient food consumption, and climate mitigation may be effective to improve water quality. These scenarios may also contribute to other SDGs. Our results may suggest directions for future policies for meeting SDGs in China. Based on our analysis, we may conclude that it is important for such policies to consider the following:

\section{i. $\quad$ Taking appropriate indicators for SDGs 6 and 14}

Following the current national plan on the implementation of SDGs, we took the Chinese "Environmental Quality Standard for Surface Water" for "grade III" water as the standard for good ambient water in SDG 6. According to this standard, the concentration of $\mathrm{NH}_{3}$ in rivers may not exceed $1.0 \mathrm{mg}-\mathrm{N} / \mathrm{L}$, and TP may not exceed $0.2 \mathrm{mg}-\mathrm{P} / \mathrm{L}$. This standard, however, does not provide information on the concentrations of other $\mathrm{N}$ forms (e.g., nitrite, nitrate, total nitrogen). This might 
lead to a biased assessment of water quality in practice. For example, water in some river sections may have low concentrations of $\mathrm{NH}_{3}$ but high concentrations of other $\mathrm{N}$ forms that can lead to eutrophication. Water quality in these river sections might then be qualified as good if only based on the $\mathrm{NH}_{3}$ concentrations. Therefore, appropriate indicators for $\mathrm{N}$ pollution in rivers is needed for China. Such indicators could be developed by improving the current water quality standards in China. For example, concentrations for other nutrient forms could be added. Differentiating the standards for water bodies based on their purpose of use (e.g., drinking water, irrigation water and etc.) may also be useful for the implementation at the regional scale. Examples of such standards from other countries or world regions (e.g., the United States, European Union) are summarized in Table C.1 in Appendix VI.

China does not have a specific indicator (e.g., standards for nutrient concentrations) to monitor the changes in coastal eutrophication for SDG 14. We used in this study the ICEP indicator to assess the potential of coastal eutrophication. We realized that there are some limitations related to ICEP. For example, ICEP is calculated on an annual basis and thus does not consider the seasonality of coastal eutrophication. Nevertheless, this indicator could be adopted as the first step to indicate the potential for harmful algal blooms in China.

\section{ii. Trade-offs and synergies between SDGs}

There are trade-offs and synergies between SDGs 6 and 14, and the other SDGs. Policies that lack consideration of the trade-offs and synergies may meet SDGs 6 and 14 at the expense of some other SDGs (Figure 7.7). One example is the recent Chinese policy on banning livestock production in the so-called Non-Livestock Production Regions (NLPRs) (SCPRC, 2015; Wang et al., 2018a). The aim of this policy is to control water pollution in the vulnerable water bodies by moving pig production from NLPRs to southwest and northeast provinces. This policy is estimated to reduce nutrient losses to waters by up to $27 \%$ for $\mathrm{N}$, and $48 \%$ for P in southeast China, which contributes to meeting SDG 6 in NLPRs (Bai et al., under revision). However, this policy may increase $\mathrm{N}$ losses to the air and to groundwater in the southeast and northeast, which has adverse impacts on SDGs 6 and 15 (biodiversity on land) in those regions (Bai et al., under revision). Thus, it is 
important to account the interactions between the SDGs across sectors and regions in future policies to increase positive synergy effects, and to avoid adverse impact of policies for SDGs 6 and 14 on the other SDGs. Our most effective scenario $\mathrm{AG}+\mathrm{SE}+\mathrm{SFC}+\mathrm{CLI}$ is an example of such future policies that contribute simultaneously to the achievement of SDGs 6 and 14, and other SDGs.

We explored future scenarios to reduce nutrient pollution in rivers and coastal waters of China using the MARINA 2.0 model. Uncertainties in our results are mainly related to the model approach and the assumptions in the scenarios. There are some limitations to the model approach of MARINA 2.0. For example, the MARINA 2.0 model does not consider N and $\mathrm{P}$ accumulation in the soil and sediments over time (Strokal et al., 2016a; Wang et al., Under Review). This accumulation is important especially for $\mathrm{P}$ because of its strong adsorption/desorption process (Beusen et al., 2016; Sharpley et al., 2013), and can lead to delays in the effects of the management options (Strokal et al., 2017). However, the dominant sources of $\mathrm{P}$ inputs to rivers are point sources rather than diffuse sources in China. The study of Strokal and de Vries (2012) shows that point sources have larger effects on river export of nutrients than including $\mathrm{P}$ accumulation in the soil for Chinese rivers. Thus this limitation does not influence our main conclusion to a large extent. There are other uncertainties related to the model approach, such as model inputs and parameters. Wang et al. (Under Review) have validated the MARINA 2.0 model for several large rivers in China. This builds trust in using the model.

In addition, there are limitations to our scenarios, related to the assumptions for the future. For example, we assume that sewage treatment will reach an optimal level according to existing technologies (Khiewwijit, 2016; Van Puijenbroek et al., 2019). We assume that all manure will be recycled in agriculture or will be treated as wastewater. Many technologies such as anaerobic digestion and composting are available to efficiently recycle or treat manure (Burton and Turner, 2003; Chadwick et al., 2015; Liu et al., 2018b; Loyon et al., 2016; Yang, 2018). We realized that the full implementation of these technologies in all river basins as assumed may be challenging. We did not study the economical, practical and institutional feasibilities. However, China is expected to develop fast in economy in the coming decades (Crespo Cuaresma, 2017). Opportunities exist for development and implementation of advanced technologies. Therefore, we consider our scenario challenging to achieve, but not impossible in 2050 given also the current 
ambitious aim to meet the SDGs in 2030. Climate mitigation by countries is also assumed to be optimistic to reach a low level of climate change. This is ambitious, but we aim to explore the largest potential to reduce water pollution by climate mitigation.

\subsection{Conclusion}

In this study, we explored how future scenarios in agriculture, sewage, food consumption, and climate mitigation may affect nutrient pollution in rivers and coastal waters, and contribute to meeting SDGs 6 and 14. We quantified total dissolved N (TDN) and P (TDP) inputs to six large rivers, and river export of TDN and TDP to seas at the sub-basin scale in 2012 and 2050 using the MARINA 2.0 model. For 2050, we took the SSP5-RCP8.5 (Shared Socio-economic Pathway 5 - Representative Concentration Pathway 8.5) from Wang et al. (Under Review) as the baseline scenario. Based on SSP5-RCP8.5, five alternative scenarios were developed named SE (improved sewage treatment), AG (improved nutrient use efficiencies in agriculture), $A G+S E$ (combination of $A G$ and $S E$ ), $\mathrm{AG}+\mathrm{SE}+\mathrm{SFC}$ (sustainable food consumption in addition to $\mathrm{AG}+\mathrm{SE}$ ), $\mathrm{AG}+\mathrm{SE}+\mathrm{SFC}+\mathrm{CLI}$ (climate mitigation in addition to $\mathrm{AG}+\mathrm{SE}+\mathrm{SFC}$ ).

Our results show that it is difficult to meet SDGs 6 and 14 in the baseline scenario (SSP5RCP8.5). In SSP5-RCP8.5, nutrient pollution in rivers and coastal waters will remain at high levels until 2050. We projected 18,612 kton of TDN, and $1327 \mathrm{kton}$ of TDP from land to rivers in 2050. As a result, river water at 23 out of the 26 sub-basin outlets do not meet the water quality standard for SDG 6 in China. About 4299 kton (TDN) and 373 kton (TDP) of these nutrients are transported by rivers to coastal waters. This will likely result in high potentials for coastal eutrophication by all six rivers (ICEP $>0$ ), and makes it difficult to meet SDG 14.

SDGs 6 and 14 may be met in our alternative scenarios. For example, AG+SE+SFC+CLI is found to be the most effective scenario and will likely reduce nutrient inputs to rivers by 64\% (TDN), and 90\% (TDP) in 2050 compared to SSP5-RCP8.5, contributing to SDG 6. We estimated water quality at all sub-basins meet the water quality standard based on TDP concentrations at their outlets, whereas only outlets of 5 sub-basins may have DIN concentrations meeting the standard. Thus that one of the challenges to meet SDG 6 for China is to reduce $\mathrm{N}$ pollution in rivers. In this scenario, river export of nutrients to seas could be 68\% (TDN) and 91\% (TDP) lower than in SSP5-RCP8.5. The potential for coastal eutrophication is therefore much lower than in SSP5-RCP8.5, contributing to SDG 14. Huai, 
Yangtze, and Pearl rivers are estimated to cause low potentials for coastal eutrophication. However, the Liao, Hai and Yellow rivers draining into the Bohai Gulf may still have high potentials for coastal eutrophication.

Future scenarios that contribute to SDGs 6 and 14 may also contribute to other SDGs. Our results show that effective future scenarios for SDGs 6 and 14 include improved nutrient management in agriculture and sewage systems, healthy and efficient food consumption, and climate mitigation. These scenarios may thus also contribute to meeting other SDGs such as SDG 2 - "zero hunger", SDG 11 - "sustainable cities and communities", SDG 13 "climate action" and etc.

Our study may help in formulating future policies to ensure good water quality in rivers and coastal waters, and to meet relevant SDGs. Our alternative scenarios suggest directions for future policies to meet SDGs 6 and 14 in China. When formulating such policies in China, it is important to consider 1) taking appropriate indicators for SDGs 6 and 14, and 2) trade-offs and synergies between SDGs. We discussed the strengths and weaknesses of the indicators for SDGs used in this study. They could be adopted for China with some further improvement. Our most effective scenario AG+SE+SFC+CLI is an example of such future policies that contribute simultaneously to the achievement of SDGs 6 and 14, and other SDGs.

\section{Acknowledgments}

This study is funded by the National Key R\&D Program of China [project number 2016YFE0103100], the WIMEK (Wageningen Institute for Environment and Climate Research) fellowship [project number 5160957306], and the KNAW (Koninklijke Nederlandse Akademie van Wetenschappen) project SURE+ [project number PSASA-E$01]$. 



\section{Chapter 8. Discussion and Conclusions}

In this chapter, a general discussion and conclusions of the research in this thesis are presented. First, I summarize the main findings of the thesis in Section 8.1. Next, I reflect on the methodology used in this thesis, focusing on the modeling approach (Section 8.2), indicators for nutrient pollution in water systems (Section 8.3), and scenarios analysis (Section 8.4). Finally, in Section 8.5, I give a future outlook by drawing important lessons for nutrient modeling in general, and by discussing the implications of this thesis for future policies for improving water quality in China.

\subsection{Nutrient pollution in water systems in China: Objectives and main findings of this thesis}

The objective of this $\mathrm{PhD}$ thesis is to improve our understanding of nutrient pollution in water systems in China, with a focus on the challenges, trends, and solutions. To this end, I formulated six sub-objectives for my research on nutrient pollution in water systems (sub-objectives 2, 4 and 6) from human activities on land (sub-objectives 1, 3 and 5).

Sub-objectives focusing on the challenges:

1. To identify hotspots for nutrient losses from food production (Chapter 2)

2. To compare nutrient loads to Lake Taihu with the critical nutrient loads of the lake (Chapter 3, case study)

Sub-objectives focusing on the trends:

3. To analyze how socio-economic development affects future nutrient losses from food production (Chapter 4)

4. To analyze how global change affects future river export of nutrients to seas (Chapter 5)

Sub-objectives focusing on the solutions:

5. To explore how current and improved nutrient management affects future nutrient losses from food production (Chapter 6)

6. To explore future scenarios to reduce nutrient pollution in water systems and to meet the SDGs (Chapter 7)

These objectives have been addressed in six scientific papers, resented in Chapters 2-7. The main findings and novelties of Chapters 2-7 are discussed in Sections 8.1.1 to 8.1.3) with the focus on the challenges, trends, and solutions for nutrient pollution in water 
systems in China. Figure 8.1 shows an overview of the research presented in this thesis, including the main conclusions from the chapters.

\subsubsection{Main findings focusing on the challenges}

\section{Chapter 2:}

- In the recent past, nutrient losses from food production increased dramatically in China

- Hotspots covered less than $10 \%$ of the Chinese land area, but contributed by more than half to the $N$ and P losses to the environment in 2012

This chapter provides novel insights in hotspots for $\mathrm{N}$ and $\mathrm{P}$ losses to the environment from food production based on a newly developed county scale modeling approach. Between 1990 and 2012, the area of hotspots for nutrient losses to the environment from food production expanded by a factor of 3 for nitrogen $(\mathrm{N})$, and 24 for phosphorus (P) at the county scale. In 2012, hotspots covered less than $10 \%$ of the Chinese land area but contributed by more than half to the $\mathrm{N}$ and $\mathrm{P}$ losses to the environment. Most of the hotpots were distributed in the North China Plain. Direct discharge of animal manure was an important cause of $\mathrm{N}$ and $\mathrm{P}$ losses in the hotspots. The hotspots were found to have more intensive agricultural production, a higher number of people working in agriculture, and higher incomes of farmers than in other counties. This hotspot analysis shows that region-specific nutrient management is needed to reduce nutrient pollution in China.

\section{Chapter 3:}

- To meet critical nutrient loads, river export of total dissolved $N$ and P to Lake Taihu needs to be reduced by $46-92 \%$

- Opportunities for reducing nutrient pollution in Lake Taihu are reducing synthetic fertilizer and improving wastewater treatment

The main novelties of this chapter are the analyses of sources of river export of different nutrient forms to Lake Taihu in relation to the critical nutrient loads based on a newly developed sub-basin modeling approach. Rivers exported $61 \mathrm{kton}$ of total dissolved N (TDN), and 2 kton of total dissolved P (TDP) to Lake Taihu in 2012. To meet the critical nutrient loads, this amount needs to be reduced by $46-92 \%$, depending on the desired level of chlorophyll-a. More than half of the river export of nutrients to Lake Taihu was from human activities in agriculture and urbanization. Diffuse sources contributed $90 \%$ to TDN with a relatively large share of synthetic fertilizers. Point sources contributed $52 \%$ to TDP with a relatively large share of 
sewage. Therefore, reducing $\mathrm{N}$ inputs from synthetic fertilizers and $\mathrm{P}$ from sewage may be sufficient to meet the least strict critical loads. Meeting the most strict critical loads needs a combination of reductions in diffuse and point sources, for which a further scenario analysis can be done to explore the possibilities.

\subsubsection{Main findings focusing on the trends}

Chapter 4:

- Nutrient losses from food production to water systems in the future may increase by up to $65 \%$ relative to today if nutrient management does not improve

The novelties of this chapter include the assessment of future nutrient losses to the environment from food production for different socio-economic development in the SSPs. Reactive N (Nr) losses from food production will likely increase by 2050 if nutrient management does not improve. I explored future trends in losses of $\mathrm{Nr}$ from China's food system based on the Shared Socio-economic Pathways (SSPs). In the SSPs with limited improvements in nutrient management (SSPs 3 and 4), Nr losses to water systems from food production may be $22-65 \%$ higher in 2050 than in 2010. Food production in SSP5 takes a more conventional path and slightly improved nutrient management. Nr losses in SSP5 is thus slightly lower than today's level. Potential SSPs to ensure food security with relatively low nutrient pollution in water systems may be SSPs 1 and 2, indicated by the lower Nr losses (30-71\% lower in 2050 than in 2010).

\section{Chapter 5:}

- Climate change makes water pollution control in China more difficult

This chapter is novel in providing insights on how socio-economic and climatic changes together may affect coastal water pollution in China. In 2050, coastal waters may be considerably more polluted or considerably cleaner than today depending on socio-economic and climate change. I explored future trends in river export of nutrients for the Shared Socio-economic Pathways (SSPs) and Representative Concentration Pathways (RCPs). River export of TDN and TDP is projected to be 52\% and 56\% higher in 2050 than in 2012, respectively, in SSP3-RCP8.5 assuming high challenges towards sustainable socio-economic development and severe climate change. River export of TDN and TDP is projected to be $56 \%$ and $85 \%$ lower in 2050 than today in SSP1-RCP2.6 assuming sustainable socio-economic development and low climate change. Climate change alone may increase river export of nutrients by 
24\% for TDN, and 16\% for TDP between 2012 and 2050, given the same trends in socio-economic development (SSP2-RCP8.5 versus SSP2-RCP2.6). The ICEP (Indicator for Coastal Eutrophication Potential) values for 12 Chinese rivers are calculated to assess their potential to cause coastal eutrophication in the future.

\subsubsection{Main findings focusing on the solutions}

Chapter 6:

- Current policies aimed at zero growth in fertilizer use are not very effective in reducing nutrient pollution from food production in China

The main novelty of this chapter is the insight in the effectiveness of current policies on reducing water pollution from food production in China. In 2050, N and P use efficiencies are about the same or a few percents higher in ZF (Zero growth in Fertilizer use after 2020) than in a BAU (Business As Usual), varying among provinces in China. The associated $\mathrm{N}$ and $\mathrm{P}$ losses to waters are $8-16 \%$ lower than in BAU at the national level, but still higher than today. Therefore, current policies aimed at zero growth in fertilizer use are good starts, but not very effective in reducing nutrient pollution from food production in China. To further reduce $\mathrm{N}$ and $\mathrm{P}$ losses from food production, additional options are suggested such as reducing fertilization without yield losses, better manure management, and reducing nutrient excretion in animal production.

\section{Chapter 7:}

- SDGs 6 and 14 can be met with improved nutrient management in agriculture and sewage systems, efficient food consumption, and climate mitigation

- Future scenarios contributing to SDGs 6 and 14 may also contribute to other SDGs

This chapter is novel in exploring future scenarios to achieve the Sustainable Development Goals (SDGs) for improved river and coastal water quality in China. SDGs 6 for "clean water and sanitation" and 14 for "life below water" are difficult to meet in the baseline scenario (SSP5-RCP8.5). In 2050, 23 of the 26 sub-basins do not meet SDG 6 at the sub-basins' outlets. Six large rivers do not meet SDG 14, with high potential for coastal eutrophication at the river mouths. Future scenarios combing improved nutrient management in agriculture and sewage systems, healthy and efficient food consumption, and climate mitigation may be effective to meet SDGs 6 and 14 . Following these scenarios, nutrient inputs to rivers may be reduced by $64 \%$ for TDN 
and $90 \%$ for TDP in 2050 compared to the baseline scenario. As a result, more than $30 \%$ of the sub-basins of six large rivers in China may be able to meet SDG 6 at their outlets, indicated by low $\mathrm{N}$ and $\mathrm{P}$ concentrations. River export of nutrients is projected to be $68 \%$ and $91 \%$ lower for TDN and TDP than in the baseline scenario. Therefore, three large rivers (the Huai, Yangtze, and Pearl rivers) may meet SDG 14 with low potential to cause coastal eutrophication, indicated by negative ICEP values. Future scenarios that contribute to SDG 6 and 14 may also contribute to other SDGs, focusing on zero hunger (SDG 2), responsible consumption and production (SDG 12), climate action (SDG 13) and others. 

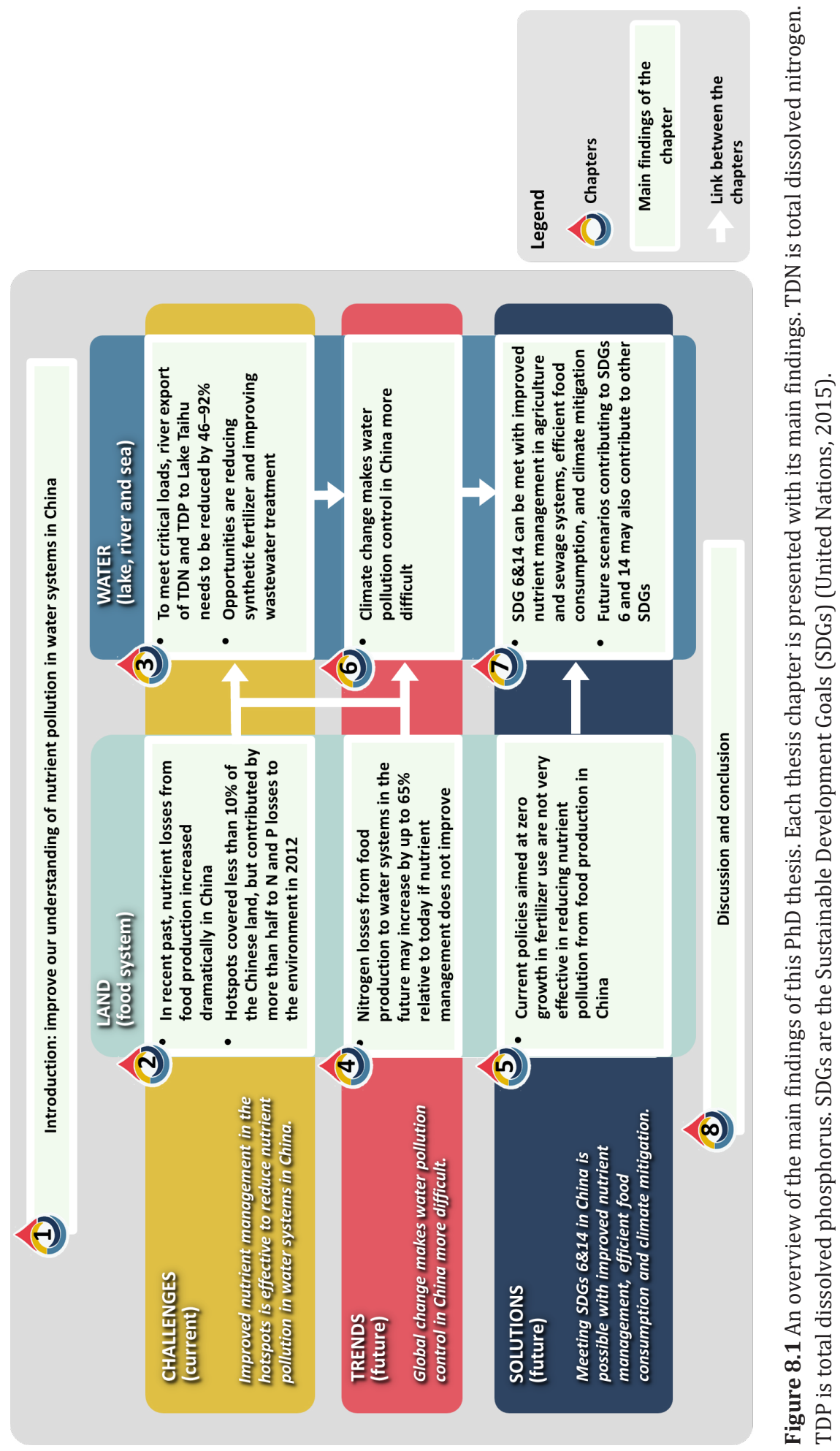


\subsection{Modeling nutrient pollution in water systems in China}

In this section, I first reflect on the modeling approaches applied in this thesis, with a focus on why NUFER and MARINA models were selected. I discuss both models' strengths (Section 8.2.1) and weaknesses (Section 8.2.3). Next, I discuss the NUFER and MARINA models' performance, following a step-wise approach for model evaluation (Section 8.2.2). Last, suggestions for future improvements in the modeling approaches are given based on their weaknesses (Section 8.2.3).

\subsubsection{Modeling approach}

\section{NUFER (NUtrient flows in Food chains, Environment and Resources use)}

To better understand the impact of human activities in food production on nutrient pollution in water systems, I used the NUFER model. The original NUFER model was developed to quantify past and future $\mathrm{N}$ and $\mathrm{P}$ flows in the food chain for 31 provinces and China (Ma et al., 2010; Ma et al., 2013a; Ma et al., 2013b). Based on the original model, Zhao et al. (2017) developed and applied a NUFER-farm model for farms in the North China Plain. This thesis is the first to develop and apply a new NUFER-county model for all counties in China for the years 1990, 2000, 2012, and 2050. Table 8.1 summarizes the characteristics of different versions of the NUFER model.

Many models exist for China to quantify nutrient flows in food production at regional and national scales (Chen et al., 2008; Cui et al., 2013; Gu et al., 2015; Gu et al., 2012; Liu et al., 2004; Ma et al., 2012a; Ti et al., 2012) (see Table 1.1 in Chapter 1 for examples of these models). I chose the NUFER model for research in this thesis given its following strengths:

○ The food chain approach

NUFER is the first to model nutrient losses from the food production-consumption chain in China using a food chain approach (Ma et al., 2010). The food chain consists of four compartments: crop production (18 crop categories), animal production (12 animal categories), food processing, and food consumption. For each compartment, an inputoutput balance is calculated. This balance includes 1) $\mathrm{N}$ and $\mathrm{P}$ inputs to the food chain via synthetic fertilizers, animal manure, biological $\mathrm{N}_{2}$ fixation, atmospheric deposition and etc; 2) N and P outputs from the food chain via food products (e.g. crop yield and animal meat) and losses to the air and water systems; and 3) $\mathrm{N}$ and P cycling within the food chain among different compartments via crop and animal products, residues, animal 
manure, and human excreta. The food chain approach makes NUFER flexible for studying $\mathrm{N}$ and $\mathrm{P}$ losses in each compartment and the entire food chain, with clear system boundaries. Based on the modeled nutrient balance, $\mathrm{N}$ and $\mathrm{P}$ use efficiencies in the food chain are also calculated, indicating the $\mathrm{N}$ and $\mathrm{P}$ cost of food production and consumption. A few other models also quantify $\mathrm{N}$ and $\mathrm{P}$ flows in part of the food productionconsumption system in China (Chen et al., 2008; Cui et al., 2013; Gu et al., 2015; Gu et al., 2012; Liu et al., 2004; Ti et al., 2012). However, these models do not always apply clear system boundaries. And they focus on either N or P, but not on both elements, as NUFER does.

○ Multiple spatial scales

After further development in this thesis, NUFER is able to quantify past and future $\mathrm{N}$ and P losses to water systems for farms, counties, provinces, and China, in general. Most of these are all important administrative scales upon which decisions by the governments regarding socio-economic development and food production are often made. This makes NUFER a powerful tool to support effective nutrient management in food production of China at regional and national scales. For example, applying NUFER to provinces and China, as a whole, can help to assess potential nutrient management options or policies at the provincial and national levels. Using NUFER at the county scale can help to formulate region-specific nutrient management with a focus on those hotspots where nutrient losses are higher than in other counties. Applying NUFER to farms will broaden the understanding of $\mathrm{N}$ and $\mathrm{P}$ use efficiencies in different types of farms (e.g., industrial animal farms vs. smallholder animal farms), and assess potential nutrient management at the farm level. Most of the existing nutrient cycling models are available for China at the national level (Chen et al., 2008; Cui et al., 2013; Gu et al., 2012; Liu et al., 2004; Ti et al., 2012). A few models are available for other scales. For example, the Urban Rural Complex N Cycling (URCNC) model (Gu et al., 2015) quantifies an N budget for China at a $1 \mathrm{~km} \times 1$ $\mathrm{km}$ resolution. However, none of these models covers the multiple scales in NUFER from production (farm) to administrative scales (county, province, country). 


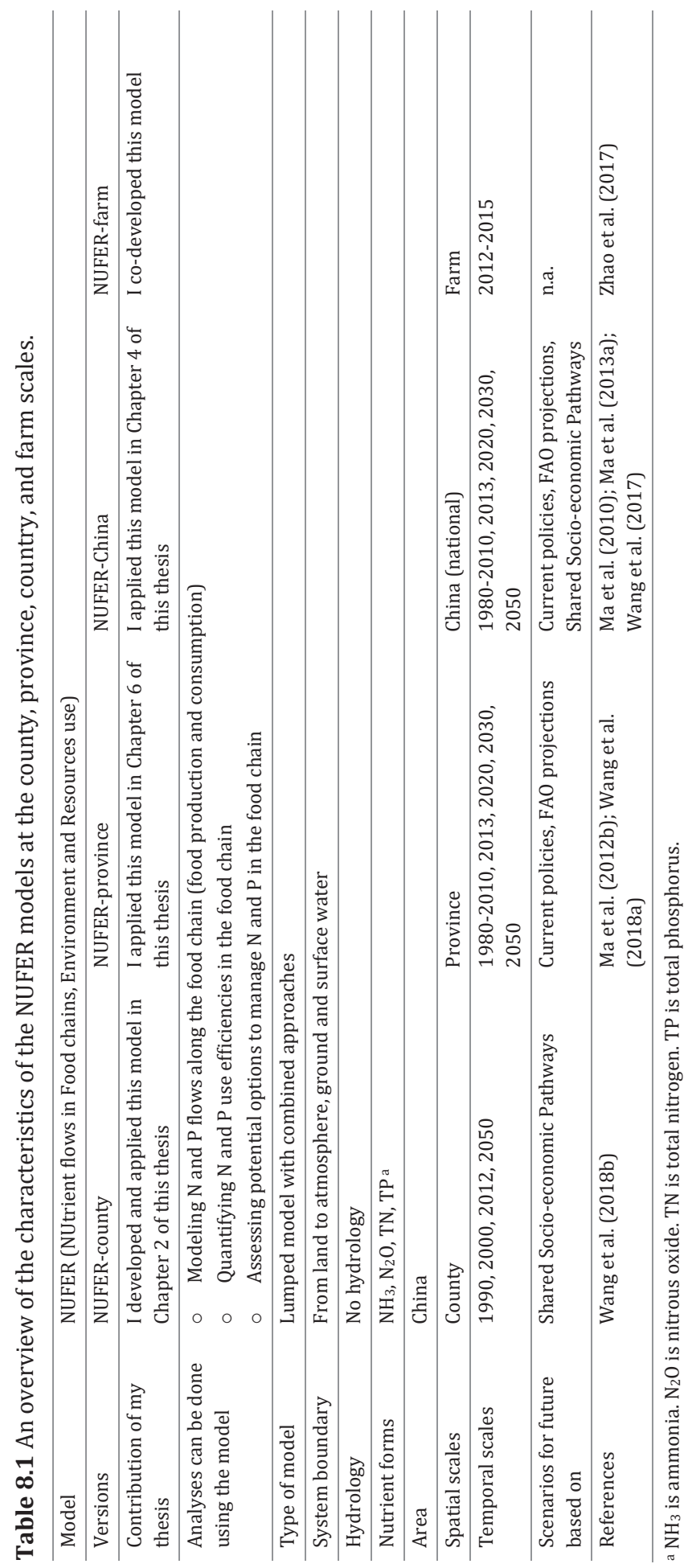




\section{MARINA (Model to Assess River Inputs of Nutrients to seAs or lAkes)}

To better understand nutrient flows from land to sea in China, I developed and applied the MARINA 2.0 model. MARINA 2.0 models current and future river export of total dissolved N (TDN) and P (TDP) by 16 Chinese rivers, by sources, at the sub-basin scale. MARINA 2.0 was developed by linking MARINA 1.0 and NUFER-county, and with information from other sources (see Annex IV for model description).

MARINA 2.0 differs from MARINA 1.0 in several ways. First, MARINA 2.0 quantifies the recent or current (2012) river export of nutrients, whereas MARINA 1.0 was developed for the past $(1970,2000)$ (Strokal et al., 2016a). However, both models are available for quantifying future (2050) river export of nutrients. A novel aspect of MARINA 2.0 is that it quantifies how socio-economic development and climate change together affect river export of nutrients based on the SSPs (O'Neill et al., 2014) and RCPs (Van Vuuren et al., 2011). To do this, the hydrology (e.g., runoff and river discharge) in MARINA 2.0 is updated based on simulations in the VIC (Variable Infiltration Capacity) model (Van Vliet et al., 2016a; Van Vliet et al., 2016b) that allows for RCPs analysis. MARINA 1.0 is mainly based on the Millennium Ecosystem Assessment (MEA) scenarios (Alcamo et al., 2005), focusing on socio-economic development. MARINA 1.0 takes hydrology from WBM (Water Balance Model) (Fekete et al., 2010), which is not available for RCPs.

Second, MARINA 2.0 uses information that better presents Chinese situations. MARINA 2.0 inputs are largely based on models for China and Chinese statistics, whereas MARINA 1.0 inputs are largely based on national estimates by global models or international statistics (Bouwman et al., 2009; Fekete et al., 2010; Van Drecht et al., 2009). This holds for the model inputs of MARINA 2.0 for 1 ) diffuse sources (e.g., use of synthetic fertilizers, animal manure) based on the nutrient balances in food production from the NUFER model at the county scale (Wang et al., 2018b), and 2) point sources (e.g., population, sewage connection and treatment) mainly based on Chinese statistics.

Third, MARINA 2.0 includes an additional source of nutrient losses to water systems: human waste from the rural population that is connected to sewage systems. This source was not included in the MARINA 1.0 model because of the Chinese rural population's limited connection to sewage systems in 2000. Last, the approach to quantify human waste is updated based on the MARINA-Global model (Strokal et al., 2019). 
I also developed the MARINA-Lake model for China's Lake Taihu to analyze nutrient export by rivers to the lake in 2012 (Wang et al., 2019a). MARINA-Lake uses similar modeling approaches and inputs as MARINA 2.0 except for hydrology (e.g., runoff, and river discharge). MARINA-Lake takes hydrology simulations from the Community WATer Model (CWATM) that has a resolution of $0.083^{\circ} \times 0.083^{\circ}$ grid (Burek et al., 2017). This fine resolution was chosen because of the relatively small drainage area of the Taihu basin. Similar to MARINA 2.0, the MARINA-Lake model includes human waste from the rural population connected to sewage systems. The approach to quantify human waste has been updated based on the MARINA-Global model (Strokal et al., 2019).

Many other models exist that quantify river export of nutrients to seas in China (Arnold et al., 2012; Beusen et al., 2016; Liu et al., 2019; Mayorga et al., 2010; UNEP, 2016b) (see Table 1.2 in Chapter 1 for examples of these models). I used MARINA 2.0 and MARINALake to model nutrient pollution in water systems in China as affected by human activities because of their following strengths:

○ Identifying the causes of nutrient pollution

MARINA 2.0 and MARINA-Lake are strong in modeling the causes of nutrient pollution in three unique ways. First, the model quantifies river export of nutrients for both diffuse and point sources. Diffuse sources include N and P losses from agricultural and nonagricultural areas via the use of synthetic fertilizers, animal manure, atmospheric $\mathrm{N}$ deposition, biological $\mathrm{N}_{2}$ fixation and etc. Point sources include $\mathrm{N}$ and $\mathrm{P}$ losses via direct discharge of animal manure and collected or uncollected human waste. The MARINA 1.0 model was the first to include direct discharge of manure to rivers and uncollected human waste in modeling river export of nutrients (Strokal et al., 2016a). These two sources are important in China because of poor manure management in animal production, and limited connection to sewage systems in rural areas. Second, MARINA 2.0 and MARINALake quantify river export of nutrients for multiple nutrient forms: dissolved inorganic (DIN and DIP) and organic (DON and DOP) N and P. This is important for identifying the sources of water pollution because different forms may have different sources. For example, human waste and animal manure are often the sources of DON and DOP, whereas synthetic fertilizers are important sources of DIN and DIP (Mayorga et al., 2010; Seitzinger et al., 2005). Third, MARINA 2.0 and MARINA-Lake quantify river export of nutrients at the sub-basin scale. This allows analyzing the contributions of sub-basins to 
the total river export of nutrients. This is particularly important for the management of large river basins (e.g., the Yellow, Yangtze, and Pearl rivers) where is a lack of knowledge on the underlying spatial patterns of nutrient export.

- Combining bio-geophysical and administrative scales (linking NUFER-county and MARINA 1.0)

MARINA 2.0 and MARINA-Lake combine the bio-geophysical (sub-basin) and administrative (county) scales by coupling with NUFER-county. The MARINA 1.0 model was coupled with NUFER-province (Strokal et al., 2016a). However, the links between the two models were limited. MARINA 1.0 only used the information for Chinese provinces from NUFER to improve the modeling of animal manure and human waste from rural population. Based on the MARINA 1.0 approach, MARINA 2.0 and MARINA-Lake further take the nutrient balance in crop and animal production at the county scale from NUFERcounty. The information from NUFER-county is aggregated from the county to the subbasin scale via grids $\left(0.5^{\circ} \times 0.5^{\circ}\right.$ or $\left.0.083^{\circ} \times 0.083^{\circ}\right)$ for MARINA 2.0 and MARINA-Lake. This creates a linked MARINA-NUFER model system that combines the sub-basin and county scales. The modeled results for sub-basins provide quantitative information on the level of pollution at the bio-geophysical scale. Nutrient balances for counties show the causes (hotspots) of the pollution at the administrative scale where policy decisions are often made. Such a new model system is a useful tool for formulating region-specific policies to improve water quality in large river basins.

- Modeling impacts of socio-economic and climatic changes

MARINA 2.0 is novel in quantifying how socio-economic and climatic changes together may impact future nutrient pollution in water systems in China, taking the SSPs and RCPs as a starting point. A few modeling studies have attempted to quantify future trends in river export of nutrients in China, and have mainly focused on the impacts of socioeconomic development (Qu and Kroeze, 2010; Strokal et al., 2016a; Strokal et al., 2017). Other studies (Howarth et al., 2006; Sinha et al., 2017) have analyzed how climate change may affect nutrient levels in water systems via river discharge in other world regions (e.g., United States), but not for China. In this thesis, I implemented the SSPs and RCPs scenarios in MARINA 2.0. The results show that socio-economic developments and climate change together may strongly impact nutrient pollution in water systems in China. A novel finding 
of this thesis is that climate change, alone, may considerably increase future river export of nutrients in China.

- Indicating potential impacts of nutrient export on coastal waters

Another strong point of MARINA 2.0 is it can be used to analyze the potential impacts of nutrient export on coastal waters. This is done by quantifying the Indicator for Coastal Eutrophication Potential (ICEP) at the river mouth. ICEP indicates the potential for the new production of harmful algae in coastal waters when receiving excess nutrients over silica from rivers (Billen and Garnier, 2007). Positive ICEP values indicate high potentials for harmful algal blooms, whereas negative ICEP values indicate low potentials for harmful algal blooms. 


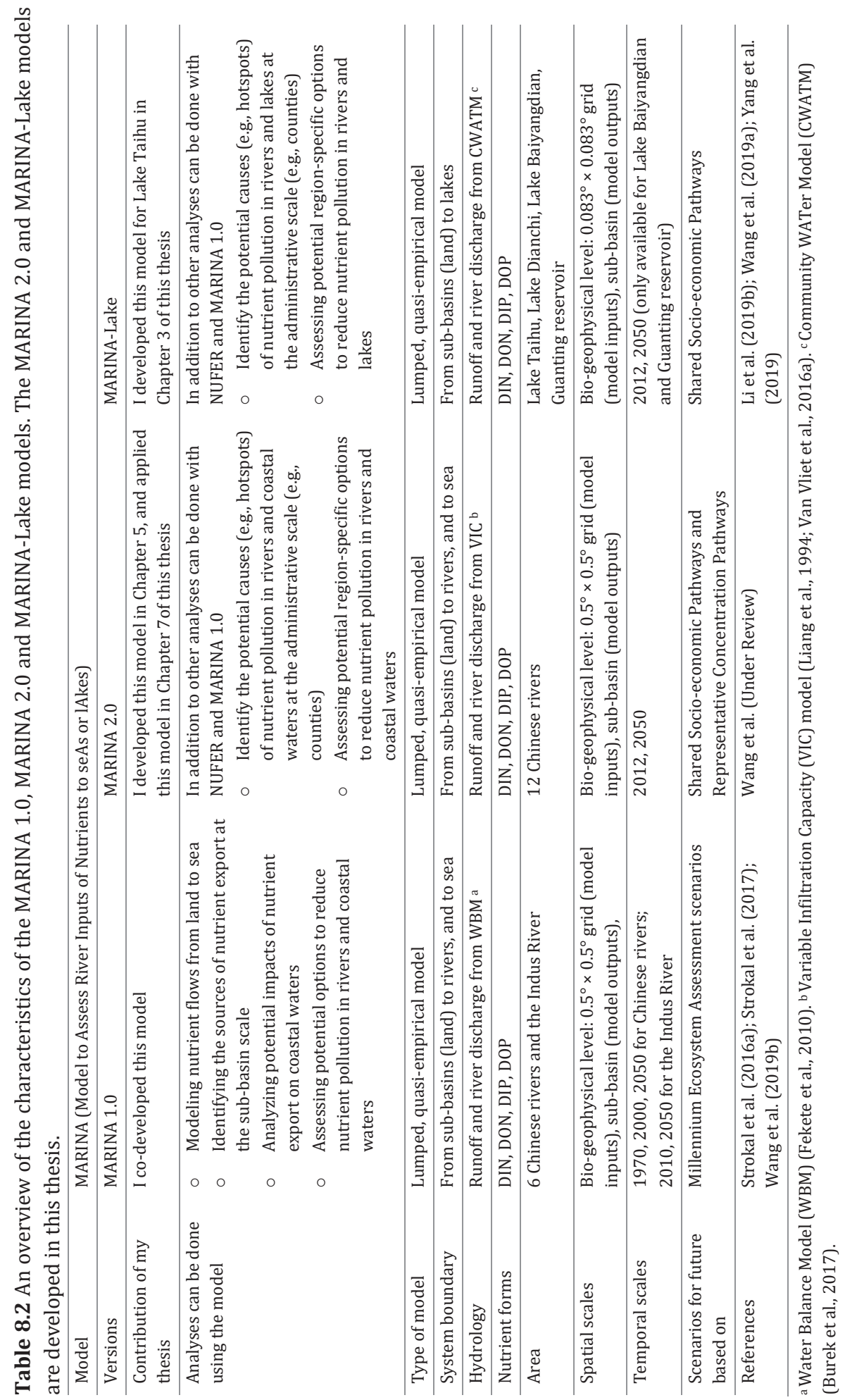




\subsubsection{Uncertainties and model performance}

Like all models, NUFER and MARINA are simplified representations of real systems. In this thesis, the uncertainties in NUFER and MARINA are mainly related to the model structure, model inputs and parameters, and the scenarios for the future. Uncertainties in the model structure are related to the main approach (equations) and the underlying assumptions for quantifying nutrient flows from land to water systems. The models presented in this thesis are based on existing modeling equations (e.g., mass balance approach in NUFER, lumped process in MARINA 2.0 and MARINA-Lake) that are commonly used and accepted in other scientific studies (Cui et al., 2013; Gu et al., 2015; Mayorga et al., 2010; Strokal et al., 2016a; UNEP, 2016b). Uncertainties also exist in model inputs and parameters. NUFER, MARINA 2.0 and MARINA-Lake take model inputs and parameters from Chinese statistics, published datasets, and other models (e.g., MARINA 1.0) that were validated for Chinese rivers (Ma et al., 2010; Strokal et al., 2016a; Wang et al., 2018b). To my knowledge, these sources are the most reliable sources for data in China. Moreover, there are also uncertainties in the scenarios for the future. For example, when implementing the SSP-RCP scenarios, I ignored the potential impacts of climate change on crop growth, which might influence nutrient flows from crop production to water systems. Being aware of the above uncertainties, it is thus important to evaluate the models' performance for China.

There are multiple ways to evaluate if the models provide sufficiently accurate representations. One of the traditional and widely used ways is to compare modeling results with observations (validation). Such validation, however, is highly dependent on the number of available measurements and their qualities (Kroeze et al., 2012). This makes it difficult to validate large scale models in regions (e.g., China) where observations on water quality are limited. Augusiak et al. (2014) introduced an "evaludation" approach that merges model evaluation and validation processes to evaluate the performance of ecological models. Strokal (2016) summarized a set of options for building trust in nutrient modeling. Based on the approaches of Augusiak et al. (2014) and Strokal (2016), I discuss the model performance for NUFER and MARINA, with emphasis on 1) comparing modeled results with measurements (validation), 2) model structure and its underlying assumptions, 3) model input data, 4) sensitivity analysis, and 5) comparing modeled results with other modeling studies. 
First, I validated the MARINA 2.0 model for Chinese rivers and the MARINA-Lake model for Lake Taihu. This was done by comparing the modeled river export of $\mathrm{N}$ and $\mathrm{P}$ to measured N and P concentrations (see Figure D.1 in Appendix VI). Three indicators were used to evaluate the result of validation: Pearson' coefficient of determination $\left(\mathrm{Rp}^{2}, 0-1\right)$, the Nash-Sutcliffe efficiency (NSE, $-\infty-1$ ) and the root mean square error to the standard deviation of measured data (RSR, $0-+\infty$ ) (Moriasi et al., 2007). According to the performance ratings from Moriasi et al. (2007), MARINA 2.0 shows a good performance with the following indicators: $\mathrm{R}^{2}$ is 0.85 , NSE is 0.72 , RSR is 0.53 . Nevertheless, it is important to realize that available measurements for model validation are very limited for Chinese rivers. Such limitation makes it challenging and difficult to validate the MARINA and NUFER models. Therefore, other ways to evaluate model performance are important and are performed.

Second, I assessed the model structure and its underlying assumptions via expert knowledge. I did this by publishing or submitting research presented in Chapters 2-7 using the newly developed NUFER-county, MARINA 2.0 and MARINA-Lake models in peer-reviewed journals. A detailed description of the model structure and its underlying assumptions are presented in the supplementary materials of the manuscripts (see model descriptions in Annex I-VI). The modeling approach and results were also presented to experts for feedback in international and Chinese conferences and symposiums. In addition, I developed the NUFER-county, MARINA 2.0 and MARINA-Lake models based on NUFER and MARINA 1.0, which have been published and widely used in scientific studies to analyze nutrient pollution in water systems in China.

Third, I assessed the model input data. NUFER mainly uses data from statistic yearbooks, existing literature, surveys, and interviews with farmers (Ma et al., 2010; Ma et al., 2012b; Wang et al., 2018b). The statistical yearbooks are, to my knowledge, the most reliable data sources in China. Making use of data from both existing literature and farmer surveys helps to avoid unrealistic model inputs and parameters. Many model parameters in MARINA 2.0 and MARINA-Lake models are from the MARINA 1.0 and Global NEWS-2 (Global Nutrient Export from WaterSheds) models. MARINA 1.0 was validated for large Chinese rivers (Strokal et al., 2016a) and the Indus River (Wang et al., 2019b). Global NEWS-2 was validated for rivers worldwide (Mayorga et al., 2010). Other model inputs and parameters are mainly from NUFER, statistic yearbooks, and published datasets (see 
Chapter 5). These sources are relatively reliable and ensure the quality of the data for running the models.

Next, I performed the sensitivity analysis. The sensitivity analysis indicates which model inputs and parameters have a large influence on model outputs. The results of the sensitivity analysis for the MARINA 2.0 model show that modeled river export of nutrients is sensitive to changes in river discharge, direct discharge of animal manure, manure excretion and use of synthetic fertilizers (see Chapter 5). This gives a better understanding of important model inputs and parameters for further checks. Such sensitivity analysis provides an example and can be applied to NUFER and MARINA-Lake models in the next steps.

Lastly, I compared the modeled results in this thesis with other modeling studies. I did this by comparing modeled results with existing NUFER or MARINA studies, as well as studies using other nutrient models. Below I discuss the results of the comparisons.

\section{Comparison with existing NUFER and MARINA studies}

Modeled results presented in this thesis are in line with existing studies using NUFER. In this thesis, NUFER quantifies increasing $\mathrm{N}$ and $\mathrm{P}$ losses from food production between 1990 and 2050 if nutrient management does not improve (Section 8.1). The increasing trends are explained by the decreasing $\mathrm{N}$ and $\mathrm{P}$ use efficiencies in food production of China. These results are in accordance with the findings of existing NUFER studies (Bai et al., 2016; Hou et al., 2013; Ma et al., 2010; Ma et al., 2012b; Ma et al., 2013a). Another important finding of this thesis using NUFER is that in the 2010s direct discharge of manure is still the main source of $\mathrm{N}$ and $\mathrm{P}$ losses to water systems from food production in China. This is also in line with previous NUFER studies (Bai et al., 2016; Bai et al., 2014b; Bai et al., 2013; Ma et al., 2019; Ma et al., 2012b; Ma et al., 2013a). For example, Bai et al. (2016) estimated that $\mathrm{P}$ losses from direct discharge of manure increased from negligible small amounts to $2.0 \mathrm{Tg}$ between 1950 and 2010.

MARINA 2.0 quantifies higher river export of TDN and TDP than MARINA 1.0 for past years (2012 versus 2000, Figure 8.2). For example, river export of TDN and TDP in 2012 in MARINA 2.0 is more than two times that in 2000 in MARINA 1.0. This is mainly explained by intensive food production and fast urbanization after 2000 in China (Bai et al., 2018b; Ju et al., 2005; Schneider, 2011; Yu et al., 2019). For example, the use of synthetic fertilizers in agriculture has increased by more than $65 \%$ between 2000 and 
2010 in China (Chen et al., 2018). The number of urban population increased by $55 \%$ between 2000 and 2012 (NBSC, 2013).

The differences in the modeled results may also be explained by the different modeling approaches and input data. The MARINA 2.0 model was developed based on MARINA 1.0 with an improved modeling approach and updated datasets. The improvements in the modeling approach are: 1) including an additional source of nutrients from rural population connected to sewage systems; 2) updating the approach to quantify human waste by adjusting the method to calculate protein N intake units of 2005 US\$ instead of 1995US\$ for gross domestic project; 3) creating new sub-basin delineation for updating hydrology based on the VIC model. The model inputs for MARINA 2.0 are mainly from NUFER and Chinese statistics, whereas MARINA 1.0 is largely based on national estimates by global models (Bouwman et al., 2009; Fekete et al., 2010; Van Drecht et al., 2009). Model validation shows that river export of TDN and TDP by MARINA 2.0 compares well with measurements (see Figure D.1 in Appendix VI). Moreover, MARINA 2.0 agrees with MARINA 1.0 that direct discharge of manure, use of synthetic fertilizers, and discharge of human waste are important sources of nutrients in water systems. Both models quantify that middle-stream and downstream sub-basins transport more nutrients to sea than upstream sub-basins. Based on the above, I consider MARINA 2.0 provides reasonable results for Chinese rivers in 2012.

MARINA 2.0 quantifies higher or lower river export of TDN and TDP than MARINA 1.0 for the future, depending on the scenarios. For example, Strokal et al. (2016a) analyzed future river export of nutrients by six large Chinese rivers in a "worst-case" based on the Global Orchestration (GO) scenario. Results showed that river export of TDN and TDP may increase by $43-80 \%$ depending on the nutrient forms between 2000 and 2050 (Figure 8.2). Assuming limited attention to sustainable development and severe climate change, MARINA 2.0 projects a higher level of pollution in 2050 for SSP3-RCP8.5 than in GO by MARINA 1.0. In contrast, river export of nutrients is $42 \%$ (TDN) and $78 \%$ (TDP) lower in SSP1-RCP2.6 than in GO in 2050 assuming sustainable socio-economic development and low climate change. These differences in 2050 are explained by the different modeling approaches (see the last paragraph) and scenarios. The GO scenario in MARINA 1.0 was developed based on the Millennium Ecosystem Assessment (MEA) scenarios (Alcamo et al., 2005). I developed the scenarios for MARINA 2.0 based on global SSP and RCP 
storylines. Despite the differences in the modeled nutrient levels, both MARINA 2.0 and MARINA 1.0 quantify that nutrient pollution in water systems in China will likely increase by 2050 if nutrient management does not improve.

MARINA-Lake shows higher river export of TDN and TDP to Lake Taihu than the critical nutrient loads, indicating a high potential for negative effects of lake eutrophication. A similar message is found for Lake Dianchi in China by applying MARINA-Lake. Li et al. (2019b) quantified that river export of TDN and TDP exceed the critical nutrient loads by 82 times and 17 times of Caohai and Waihai (part of Dianchi), respectively. Using MARINA-Lake allows for the identification of nutrient loads to the lakes by source. The main sources differ between lakes depending on the human activities in the river basins draining into the lakes (Li et al., 2019b; Yang et al., 2019). 

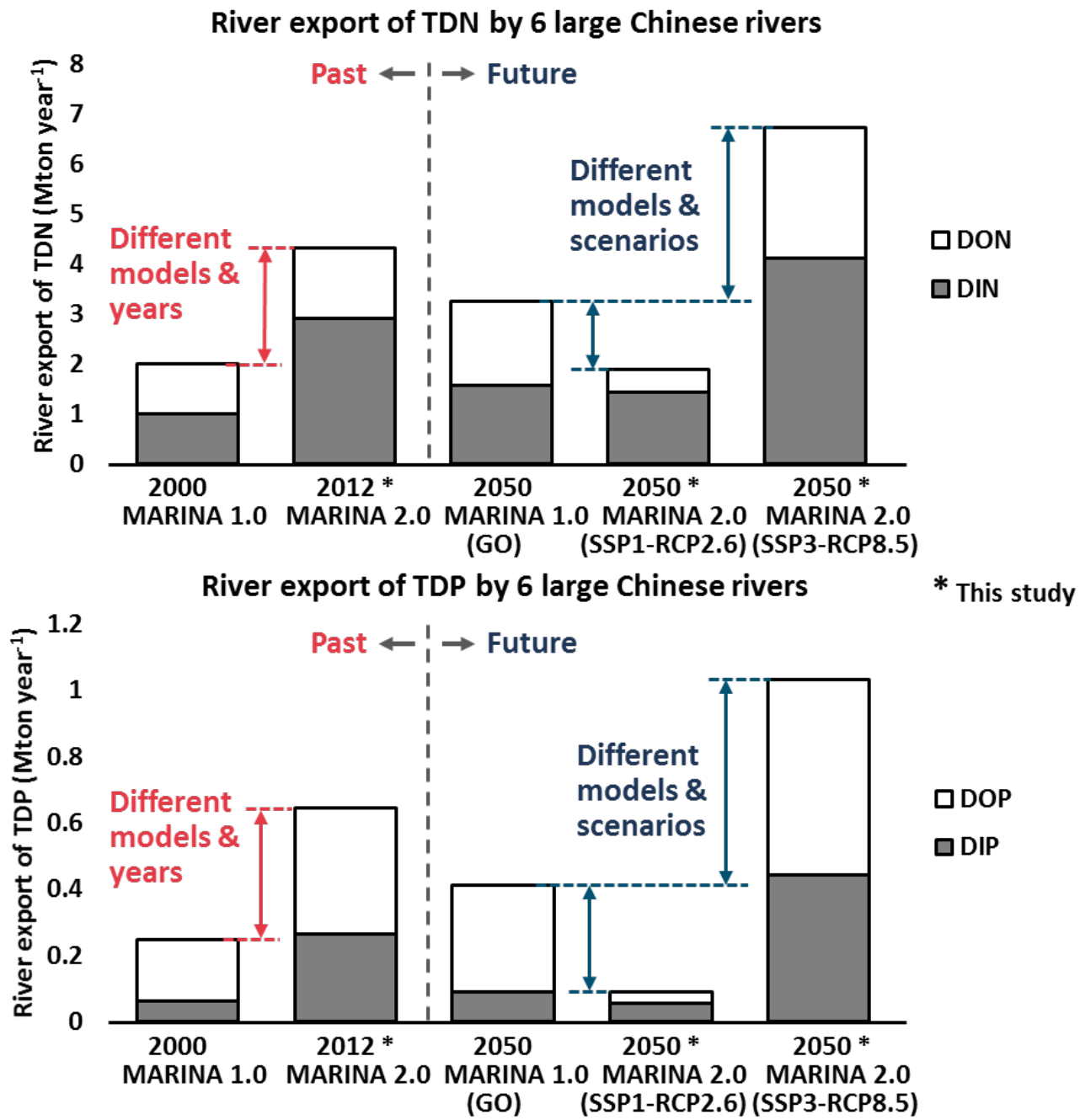

Figure 8.2 Modeled past $(2000,2012)$ and future (2050) river export of total dissolved nitrogen (TDN) and phosphorus (TDP) by six Chinese rivers using the MARINA 1.0 (Model to Assess River Inputs of Nutrients to seAs) and MARINA 2.0 models. The results of MARINA 1.0 are from Strokal et al. (2016a). The six rivers are the Yellow, Yangtze, Pearl, Liao, Huai and Hai rivers. TDN includes dissolved inorganic (DIN) and organic (DON) nitrogen. TDP includes dissolved inorganic (DIP) and organic (DOP) phosphorus. MARINA is brief for Model to Access River Inputs of Nutrients to seAs. GO is brief for the Global Orchestration scenario. SSP1-RCP2.6 is brief for a scenario combines Shared Socio-economic Pathway 1 and Representative Concentration Pathways 2.6. SSP3-RCP8.5 is brief for a scenario combines Shared Socio-economic Pathway 3 and Representative Concentration Pathways 8.5. 


\section{Comparison with studies using other models}

Many models exist to quantify nutrient flows from land to water systems in China. In this thesis, I compared the results of NUFER, MARINA 2.0 and MARINA-Lake with other modeling studies for lakes, rivers, and seas in China (see the discussion sections in Chapters 2-7). The comparisons show that modeled results in this thesis generally agree with other studies on 1) the spatial distribution of hotspots for nutrient losses to water systems, 2) the main sources of nutrients in the water systems, 3) the increasing trend in nutrient losses from food production to water systems, and 4) the increasing trend in river export of nutrients to seas.

However, there are also differences between the results in this study and other modeling studies (Crippa et al., 2016; Gu et al., 2012; Li et al., 2011a; Liu et al., 2018a; Mayorga et al., 2010; Zhou et al., 2014b) (see the discussion sections in Chapters 2-7 for details). The differences are mainly explained by the following reasons. First, the applied system boundaries are different between models in this thesis and in other studies. For example, straw is considered as the output (product) of the crop production system in NUFER. Therefore, $\mathrm{N}_{2} \mathrm{O}$ emission from the burning of straw is not considered in the model. As a result, I quantified a lower level of $\mathrm{N}_{2} \mathrm{O}$ emissions than other studies (Crippa et al., 2016; Gu et al., 2012; Zhou et al., 2014b). Another example is that NUFER only quantifies nutrient flows in the food chain, thus does not consider indirect $\mathrm{N}_{2} \mathrm{O}$ losses in the water systems. This, however, does not affect the main conclusions of this thesis because the system boundaries are clearly presented in the method sections of Chapters 2-7.

Second, the sources of nutrients are different between models in this thesis and in other studies. For example, NUFER and MARINA include direct discharge of manure, while many other models for China do not (Beusen et al., 2016; Li et al., 2011a; Liu et al., 2018a; Mayorga et al., 2010). This may results in higher estimates of nutrient levels in water systems in this thesis compared to other studies. For example, Li et al. (2011a) did not consider direct discharge of manure and thus quantified lower river export of DIP (22-25 kton of DIP) for the Yangtze river than MARINA 2.0 (128 kton) for 2012. There are also sources in other models that were not considered in the models presented in this thesis. For example, Liu et al. (2018a) included other sources of nutrients such as aquaculture, industrial wastewater, and vegetation in floodplains. These sources, however, are negligible for large river basins compared to other sources from agriculture and sewage 
(Liu et al., 2018a). Therefore, ignoring such sources does not largely influence the conclusions of this thesis.

Third, MARINA 2.0 does not quantify particulate forms of $\mathrm{N}$ and P. The ratio between particulate and dissolved nutrient forms in total $\mathrm{N}$ and $\mathrm{P}$ can differ largely among rivers (Mayorga et al., 2010; Pan et al., 2013; Yao et al., 2009). This makes it challenging to compare MARINA 2.0 results with other studies that quantify total $\mathrm{N}$ and $\mathrm{P}$ including particulate forms. Nevertheless, the results of MARINA 2.0 on dissolved nutrient forms are comparable with other studies (see model validation in Chapter 5).

Based on the above, I consider NUFER, MARINA 2.0 and MARINA-Lake appropriate for modeling nutrient pollution in water systems in China.

\subsubsection{Beyond NUFER and MARINA 2.0: future model improvements}

The modeling approaches in this thesis have a number of weaknesses, based on which research needs for model improvement are identified for NUFER, MARINA 2.0 (or -Lake) models.

- Modeling the inter-annual variations

NUFER, MARINA 2.0 and MARINA-Lake are applied on an annual basis, contributing to a better understanding of the magnitudes, sources, spatial patterns and trends in nutrient export to water systems. This annual approach, however, is limited in addressing the inter-annual variations in water pollution by nutrients. Such inter-annual variations are important because nutrient transport to rivers, lakes and coastal waters are often affected by the seasonality in human activities and climate. For example, crop production contributes different amounts of nutrients to rivers among seasons. Climate variables (e.g., river discharge and water temperature) show seasonality, and affect the retention of nutrients on land and in rivers (Basu et al., 2010; McCrackin et al., 2014; Wang et al., 2014b). A quantification of seasonal river export to seas will also provide more insights into the seasonality of eutrophication such as harmful algal blooms. Chen et al. (2019a) developed and applied the MARINA 1.1 model that accounts for seasonality to the Yangtze River to analyze river export of DIN in 2000. The seasonality approach of MARINA 1.1 can be used as an example, and be extended for seasonal simulations in other Chinese rivers and for different nutrient forms. 
Including particulate forms

MARINA 2.0 and MARINA-Lake focus on river export of dissolved forms. River export of dissolved $\mathrm{N}$ and $\mathrm{P}$ are used to calculate ICEP, indicating the potential for coastal eutrophication. I consider this as an acceptable approach, as dissolved N and P forms generally have higher bioavailability for harmful algae than particulate forms (Garnier et al., 2010). However, this approach may lead to an underestimation of nutrient pollution in water systems, especially in rivers where particulate forms are important. Future research is needed to add particulate forms to the MARINA 2.0 (or Lake) models. The module in the Global NEWS-2 model for quantifying river export of particulate $\mathrm{N}$ and $\mathrm{P}$ can be used as the basis (Beusen et al., 2005; Mayorga et al., 2010).

Including missing sources

MARINA 2.0 and MARINA-Lake include important sources such as agriculture and urbanization for nutrient losses to water systems. However, as discussed in Section 8.2, some other local sources are missing in the model. This holds for sources such as aquaculture, industrial wastewater, and direct atmospheric $\mathrm{N}$ deposition on water. Such sources may be negligible for nutrient pollution in large rivers, but can be important for analyzing local problems. For example, mining activities around Lake Dianchi are intensive and are important sources of DIP loads to the lake (Luo et al., 2004). Adding mining as the sources of $\mathrm{P}$ in the MARINA-Lake model for Lake Dianchi has helped to better understand river export of $\mathrm{P}$ to the lake (Li et al., 2019b). Therefore, including missing sources in MARINA 2.0 (or -Lake) may be needed for analyzing nutrient pollution at local scales (e.g., lakes).

○ More monitoring data for model validation and calibration

More monitoring data on nutrient flows in the food chain, and on nutrient concentrations in water systems are needed for model validation and calibration. As discussed in Section 8.2.2, validating the NUFER and MARINA models requires a large number of measurements. Such data are not always available in China. All versions of MARINA were thus validated or evaluated with limited available datasets (Li et al., 2019b; Strokal et al., 2016a; Wang et al., Under Review; Wang et al., 2019a; Yang et al., 2019). A new dataset with sufficient measurements would be valuable for validating NUFER and MARINA. Moreover, the parameters in MARINA 2.0 are largely based on the Global NEWS-2 model (Strokal et al., 2016a). A few of these parameters were calibrated on a global scale. I did 
not recalibrate the MARINA 2.0 and MARINA-Lake model for China because available monitoring data are limited. However, this does not influence the main findings of this thesis proven by the promising model performance (Section 8.2.2). Nevertheless, a more complete dataset on water quality will allow to re-calibrate the MARINA 2.0 model for China if needed.

\subsection{Indicators for nutrient pollution in water systems}

Several indicators were used in Chapters 2-7 to analyze nutrient pollution in water systems in China. These indicators help to better interpret and communicate the model results of this thesis. In this section, I discuss the indicators based on the Drivers, Pressure, State, Impact and Response (DIPSIR) framework (Kristensen, 2004) (see Figure 8.3 that presents Chapters 2-7 and the indicators in a DPSIR model of intervention framework). I reflect on the indicators, with emphasis on their strengths, weaknesses, and potential for use in assessing SDGs (Table 8.3).

O Nutrient use efficiency (Pressure-DPSIR)

Based on the NUFER model, I analyzed N and P use efficiency in food production for counties, provinces, and China as a whole (Chapters 2, 4 and 6). $\mathrm{N}$ and $\mathrm{P}$ use efficiency indicates the pressure of nutrient pollution in water systems from the perspective of DPSIR. This indicator is quantified by dividing N or P contents in the main products by $\mathrm{N}$ or P inputs to food production (Wang et al., 2018a).

The strength of this indicator is showing how much nutrients that enter food production are used efficiently by crops and animals. The rest is lost to the air and water systems. Another strength is that this indicator is flexible and can be applied for different food production systems and spatial scales. For example, in Chapter 4, $\mathrm{N}$ use efficiencies are quantified for crop production, animal production, and the whole food chain. This helps to better understand the use-efficiencies of nutrients in the whole food chain, as well as its individual parts. A weakness of adopting $\mathrm{N}$ and $\mathrm{P}$ use-efficiency is that it cannot be applied to assess nutrient pollution in water systems. The whole nutrient balance of food production must always be considered to indicate the magnitude of nutrient losses to water systems. Moreover, this indicator is difficult to measure and validate, particularly at local scales. 
Nutrient use efficiency can be used to assess SDGs such as SDG 12 - "responsible consumption and production" as it reflects whether nutrients are used efficiently in agriculture. In Chapter 7, I mainly focused on SDGs 6 - "clean water and sanitation" and 14 - "life below water" for water pollution. Therefore, I only selected indicators for quantitatively assessing these two SDGs in China. Adopting nutrient use efficiency in the future assessment can help to understand if future water pollution control options in China will contribute to meet the SDGs related to nutrient use in agriculture (e.g., SDGs 2 and 12).

- Critical nutrient loads (Impact-DPSIR)

In Chapter 3, I compared river export of TDN and TDP to lake Taihu with the critical nutrient loads. Critical nutrient loads are used as ecological thresholds, and exceeding these thresholds leads to harmful algae blooms in the lake (Janssen et al., 2017; Xu et al., 2015a). This indicates the potential impact of excessive nutrient loads to lakes. In this thesis, I took the critical nutrient loads for Lake Taihu from Janssen et al. (2017) based on the PCLake model using a food web approach.

Using critical nutrient loads allows indicating the ecological response to excessive nutrient loads to Lake Taihu. The critical loads for the same lake differ depending on the desired level of chlorophyll-a, which can be used to set resorting goals for lakes regarding its functions for usage. For example, lakes that serve as sources of drinking water must have a low level of chlorophyll-a (e.g., below $20 \mu \mathrm{g} \mathrm{Chl-a/L).} \mathrm{As} \mathrm{a} \mathrm{result,} \mathrm{the} \mathrm{critical} \mathrm{loads}$ are low for $\mathrm{N}$ and $\mathrm{P}$. There are also weaknesses in using critical nutrient loads. It is difficult to measure and validate the critical nutrient loads, particularly for lakes with spatial variation in their critical loads. Over- or underestimation of critical loads may lead to improper restoring goals for lakes. The critical loads for Lake Taihu used in this study were validated and generally agree with previous measurements and lab experiments (Janssen et al., 2017).

Critical nutrient loads indicate water quality in lakes. When assessing the SDGs for China (Chapter 7), I focused on rivers and the seas and thus did not use this indicator for lakes. Nevertheless, critical nutrient loads can be used in future research to assess SDG 6 for water quality in lakes as it shows whether the lakes receive excessive nutrient loads that can cause eutrophication. 
○ Water quality standards (State and Impact-DPSIR)

In Chapter 7, I used water quality standards as indicators to assess whether SDG 6 is achieved for rivers in China. I did this by comparing modeled $\mathrm{N}$ and $\mathrm{P}$ concentrations at the sub-basin outlets with the water quality standard of "grade III water" for rivers in "Environmental Quality Standards for Surface Water" in China (MEP, 2002). These standards provide quantitative criteria such as $\mathrm{N}$ and $\mathrm{P}$ concentrations to assess water quality. Nevertheless, water quality standards in China are only available for limited nutrient forms. For example, the "Environmental Quality Standards for Surface Water" were only for ammonia ( $\mathrm{NH}_{3}$ ) and total P (TP). This may lead to a biased assessment of water quality. For example, in some river sections water may be highly polluted with low concentrations of $\mathrm{NH}_{3}$ and high concentrations of other forms (e.g., nitrate). One will assess such river sections with good water quality only using standards for $\mathrm{NH}_{3}$. Therefore, other nutrient forms need to be considered in water quality standards. Another limitation of existing water quality standards in China is that they do not consider regional differences. Su et al. (2017) introduced a method to set nutrient standards for lakes on regional scales and identified a looser standard for the "Yungui" region. Therefore, existing water quality standards need to be improved for using them to assess SDG 6 and to support efficient water management in China. Water quality standards in developed countries, such as in the United States and the European Union, can be used as examples for the improvements (Su et al., 2017) (see Appendix VI).

○ ICEP (Impact-DPSIR)

In Chapter 5, I quantified the Indicator for Coastal Eutrophication Potential (ICEP) at the river mouth of 12 Chinese rivers using modeled results of MARINA 2.0. I also used ICEP as an indicator to assess whether China meets SDG 14 in Chapter 7. Applying ICEP contributes to a better understanding of coastal waters' ecological responses to excessive nutrient loads. The ICEP approach, however, is generic and does not consider differences in requirements of nutrients for diatoms among coastal waters. Particulate $\mathrm{N}$ and $\mathrm{P}$ forms were not considered when calculating ICEP in this thesis. ICEP is calculated using river export of nutrients on the annual basis, and thus does not consider the seasonality of coastal eutrophication. Moreover, it is difficult to measure and validate ICEP due to the complexity of harmful algae blooms. Therefore, it is important to interpret the ICEP values with care. A negative ICEP value indicates a high potential for coastal eutrophication. A 
positive ICEP value indicates a low potential, but not no occurrence of harmful algal blooms at the river mouth.

\section{Nutrient pollution in water systems in China}

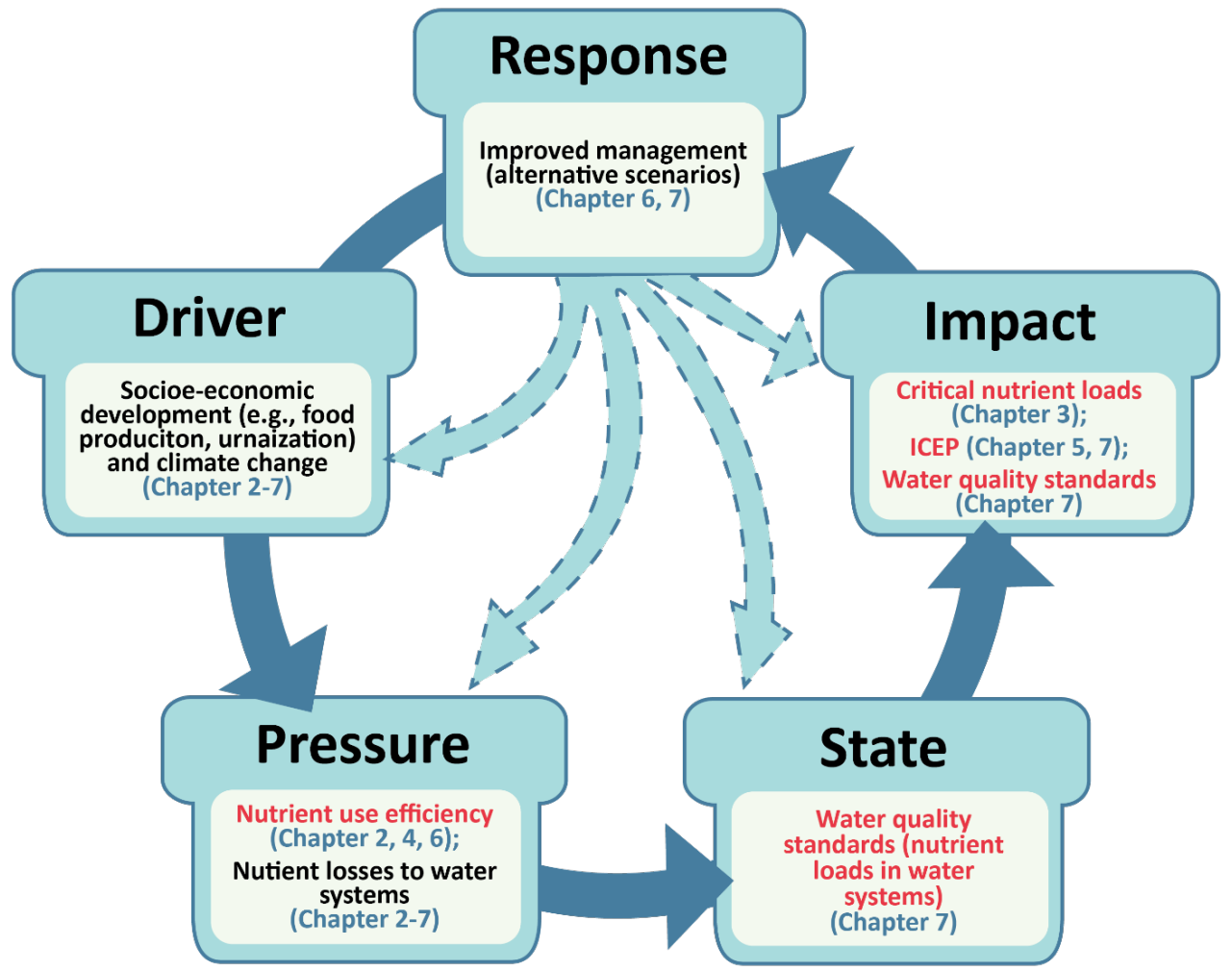

Red text refers to the indicators used in this thesis

Figure 8.3 Overview of research in this thesis presented in the Drivers, Pressure, State, Impact and Response (DPSIR) model of intervention framework. The red text refers to the indicators used in this thesis. More information on the indicators is available in Table 8.1. 


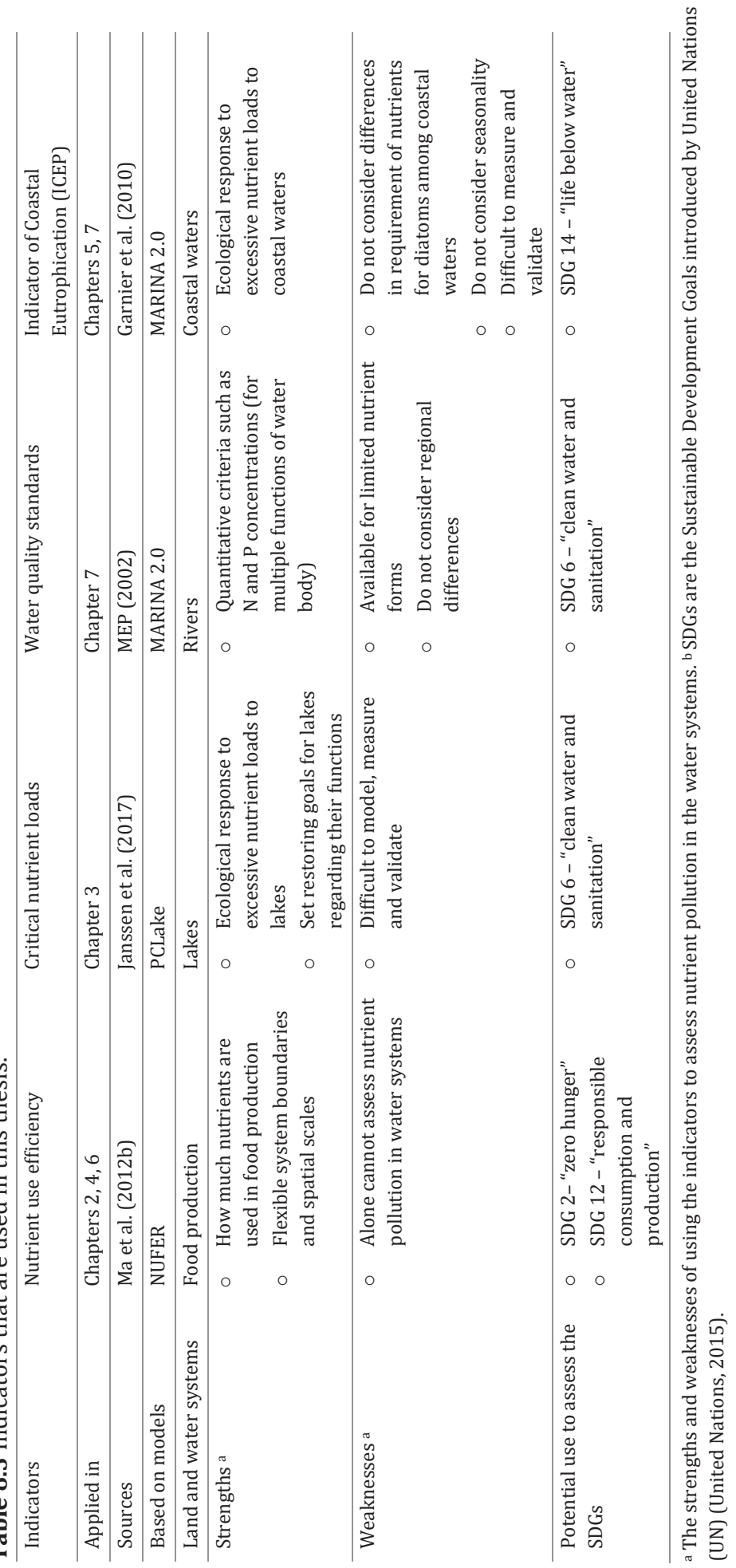




\subsection{Scenario analysis}

In Chapters 4-7, I analyzed future trends in nutrient pollution in water systems and explored potential solutions for China. For these analyses, I used the scenario analysis approach. Scenario analysis is a commonly used approach to better understand how the future may unfold, bringing together multiple disciplines (e.g., socio-economic development, environmental change) (Alcamo, 2008). It has also been proven to be a useful tool that bridges science and policy. One example of this is the use of the greenhouse gas emissions scenarios for future climate change by the Intergovernmental Panel on Climate Change (IPCC) (Nakicenovic et al., 2000). These emission scenarios have assisted the scientists and policymakers for the assessment of impacts, adaptation, and mitigation of climate change.

One can develop many types of scenarios to answer different questions for science and policy. I distinguished scenarios in three main categories based on Börjeson et al. (2006), Ferrier et al. (2016) and Maier et al. (2016): predictive, explorative and normative (Figure 8.4). In this thesis, I developed and applied predictive and explorative scenarios in Chapters 4-7.

\section{- Predictive scenarios}

Predictive scenarios are often used to answer the question: What will happen? Predictive scenarios include two different types: Forecasts and What-if scenarios. Forecasts scenarios are mostly used to analyze what will happen in the future if the likely development unfolds. This type of scenario is used in Chapter 6 to analyze future $\mathrm{N}$ and $\mathrm{P}$ losses from food production following a business as usual trend. Forecasts scenarios are often used as the baseline to build other types of scenarios (alternative scenarios). Whatif scenarios are used to analyze what will happen if some specific events take place in the near future that may have an important impact on future development. The ZF (Zero Fertilizer) scenario, which assumes zero growth in the use of synthetic fertilizers after 2020 in Chapter 6 is considered this type of scenario. ZF was developed based on recently introduced Chinese policies aiming at zero growth in synthetic fertilizer use after 2020 (MOA, 2015c). Appling the ZF scenario helps to assess the effectiveness of current policies on reducing future nutrient losses from food production to water systems in China. 
○ Explorative scenarios

Explorative scenarios respond to the questions: What can happen? There are two types of explorative scenarios: External and Strategic Scenarios. External scenarios are developed to explore what can happen under a wide range of future developments. Examples are the scenarios in Chapters 4 and 5 that combine multiple SSPs and RCPs. Results of applying these scenarios in NUFER and MARNA can help to better understand how future nutrient pollution may be affected by possible socio-economic development and climate change. Strategic Scenarios are developed to explore what can happen if certain actions are taken. This scenario type is of special interest to the users who would like to explore solutions to environmental issues. I used strategic scenarios in Chapters 6 and 7. For example, the alternative scenarios in these two chapters that incorporate potential solutions (e.g., improved nutrient management in agriculture and sewage, efficient food consumption, climate mitigation) are strategic scenarios. Results of scenario analysis in Chapters 6 and 7 provide useful insights into what potential options may be effective in reducing future nutrient pollution in water systems in China.

○ Normative scenarios

Normative scenarios answer the question: How can a desired target be reached? Two types of normative scenarios are: Preserving and Transforming scenarios. Preserving scenarios are used to answer the questions: How can a target be reached by adjusting current development. This type of scenario is useful in analyzing how a target can be efficiently (e.g., cost-efficiently, resource-efficiently) reached based on optimization models. For example, the Regional Air Pollution Information and Simulation (RAINS) model has been applied to identify optimal sets of mitigation options to reach air quality targets for Europe and Asia (Boudri et al., 2002; Schöpp et al., 1998; Wagner et al., 2006). Another example is the weighted Gini coefficient approach to allocate permits (targets) for pollution discharges based on the equality principle (Meng et al., 2008; Tian et al., 2014; WU et al., 2006; Yuan et al., 2017). Applying the preserving scenarios based on optimization in the future studies of NUFER and MARINA is useful for pollution management in China. This can, for example, help to search for the cheapest solutions to reduce nutrient losses to water systems from multiple sources.

Transforming scenarios respond to the question: How can a target be reached if necessary changes are needed in the prevailing structure? This type of scenario is needed when it is 
impossible to reach the desired target if the on-going development continues. Backcasting is a common approach here that provides results on target-fulfilling (trend break) solutions with a discussion of what changes in current systems may be needed to implement these solutions. The back-casting approach was applied to MARINA 1.0 by Li et al. (2019a) to explore potential options to meet the environmental targets for the Huang, Huai and Hai rivers. The targets aim to avoid coastal eutrophication and are derived from the ICEP approach (ICEP $<0$ to avoid harmful algal blooms). Upscaling this study for other rivers using NUFER and MARINA will help to identify options for meeting certain targets for a desired future in China.

Scenario analysis is a strong tool because it brings knowledge and concerns from scientists, policymakers, and stakeholders together. A possible way to do this is through participatory approaches (Alcamo, 2008). Involving the users (e.g., policymakers) in building the scenarios allows these users to have a shared understanding of the scenarios. This makes it more likely that they will use the scenarios for decision-making. Involving stakeholders who are often non-scientific experts allows the scenarios to take into account different perspectives and the major uncertainties. This increases the feasibility of the potential options in the scenarios for pollution management purposes. In this thesis, I developed most of the scenarios based on existing socio-economic and climate change pathways, current policies, scientific expert knowledge, and other scientific studies. I consider this appropriate, as the aim of my scenario analysis was to assess nutrient pollution in water systems for the long-term future (until 2050) for a wide range of possible future development, and to explore potential solutions to this pollution. Based on such results, further scenario analysis using a participatory approach can be done to explore short-term or long-term solutions taking into account their feasibilities (e.g., technical, economic, operational feasibilities). 


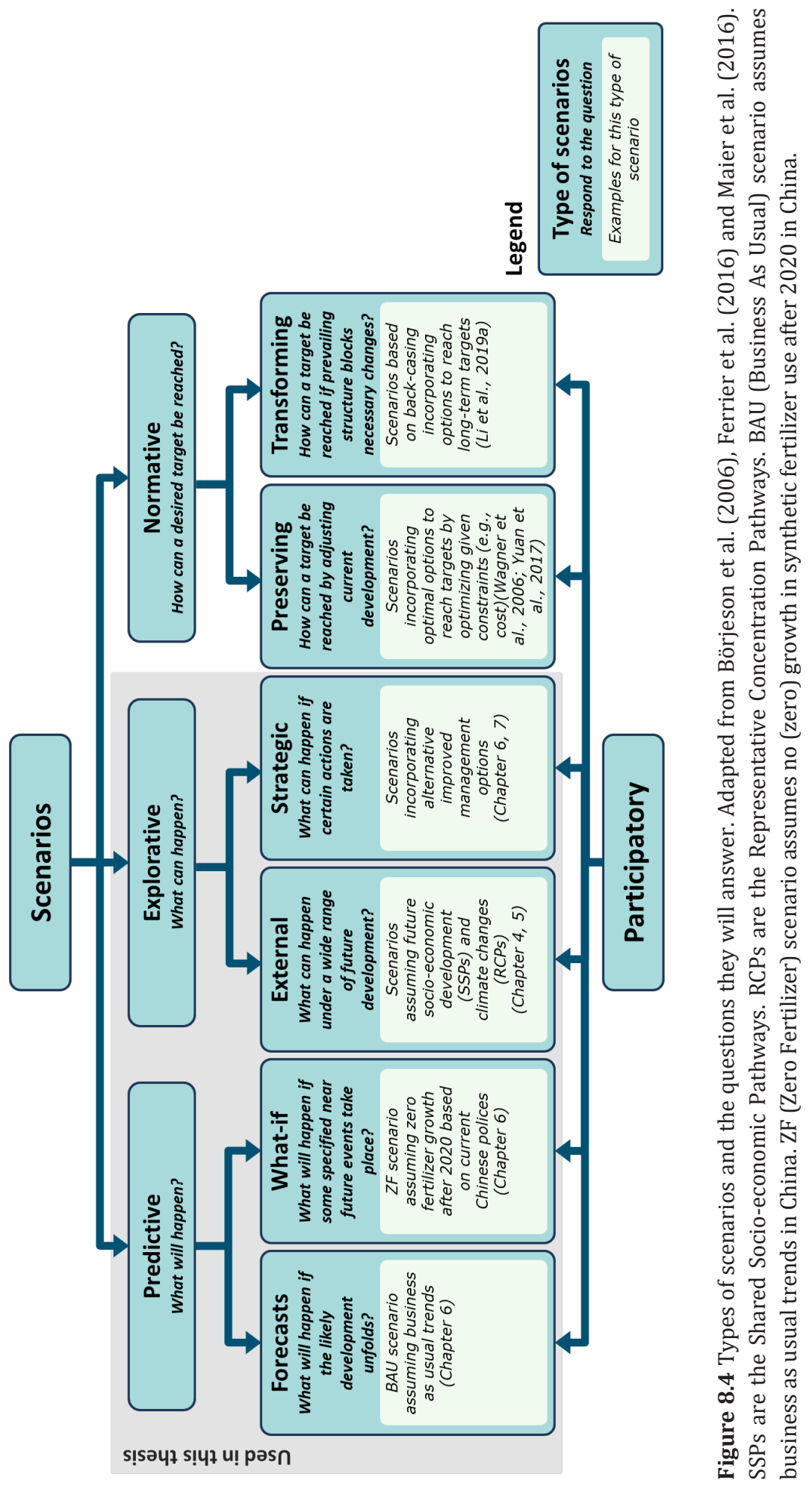




\subsection{Future outlook}

In this section, I draw the important lessons for future nutrient modeling based on the discussion in Sections 8.2-8.4. Next, I discuss the policy implications of this thesis based on changes in Chinese environmental policies in the last decades. Last, I give closing remarks on how my research will contribute to future water pollution control in China.

\subsubsection{Lessons for future nutrient modeling}

○ Linking models at administrative and bio-geophysical scales can help to explore solutions to reduce nutrient pollution in water systems

The MARINA 2.0 model is developed by linking the NUFER and MARINA 1.0 models. Linking these two models allows an analysis of nutrient pollution in water systems at both administrative (by NUFER) and bio-geophysical (by MARINA) scales. This is important since water pollution needs to be understood at the bio-geophysical scales (e.g., basin, sub-basins), but decisions on human activities that drive the pollution are often made at the administrative scales (e.g., county, province, country). The MARINA 2.0, which allows analysis on both scales, is thus useful to quantify the magnitude, identify the sources, and explore solutions to nutrient pollution.

○ The preferred (spatial) scales of modeling depending on the research objectives In this thesis, nutrient losses to water systems from food production are modeled at multiple scales, depending on the research objectives. In Chapter 2 , the research objective was to identify the hotspots for nutrient losses at a detailed spatial level, for which I performed a county scale analysis. In Chapter 4, the research objective was to analyze the future trend in nutrient pollution from food production taking the global socio-economic pathways as a starting point, for which an analysis at the national scale is appropriate. In Chapter 6, the research objective was to analyze the effectiveness of national current policies on reducing nutrient losses in food production. Such policies are introduced by the central government, and then implemented by the provinces. The differences in the effectiveness of the specific policy among provinces are analyzed based on the provincial scale analysis. Based on the above, I draw the lesson for future nutrient modeling that the modeling scales should be dependent on the research objectives. The detailed scales are not always preferable and appropriate. 
- Indicators can help to better understand and communicate the modeling results

Quantitative indicators can be used to understand and communicate the modeling results. In this thesis, I quantified several indicators based on the results of NUFER and MARINA (see Section 8.4). These indicators contribute to a better understanding of nutrient pollution in water systems in China by indicating the pressure, state and impact of this issue (see Figure 8.3). Using such indicators also makes it easier to communicate the modeling results to society. For example, ICEP shows the potential for coastal eutrophication and was used as an indicator for SDG 14. It is much easier for non-experts to understand the result of ICEP (e.g., high or low potential for coastal eutrophication) than the absolute level of river export of nutrients.

- Combing different types of scenarios is useful in exploring solutions in environmental modeling

Combining different types of scenarios in NUFER and MARINA studies is useful for analyzing future trends, and exploring solutions to nutrient pollution in water systems for China (see Section 8.4). I did this by first using the predictive scenarios to assess what will happen if current development unfolds (business as usual trends) or if current policies are implemented. Next, I developed and applied explorative scenarios to explore what can happen for a range of storylines based on SSPs and RCPs, and what can happen if alternative improved management takes place. The results show how future nutrient pollution will likely be affected by global change, and what are the potential solutions to reduce pollution. The normative scenarios based on optimization and back-casting can be used as the next step to identify optimal sets of solutions to reach the desired target (e.g., avoiding coastal eutrophication). The participatory approach that involves policymakers and stakeholders in the development of scenarios will help to increase the feasibility of the scenario for implementation.

- The modeling approach in this thesis can be used to analyze nutrient pollution in water systems in other world regions

All aforementioned lessons are useful in investigating water pollution in other world regions. One example of this is my recent application of the MARINA 1.0 model to the Indus River (Wang et al., 2019b), in which I analyzed its current and future river export of $\mathrm{N}$. Such an analysis can also be done by upscaling NUFER to other countries to analyze nutrient flows in food production. Indicators used in this thesis for China can be used for other world regions to interpret and communicate modeled results. Performing scenario analysis as I did will help to explore solutions to water pollution in other countries. 


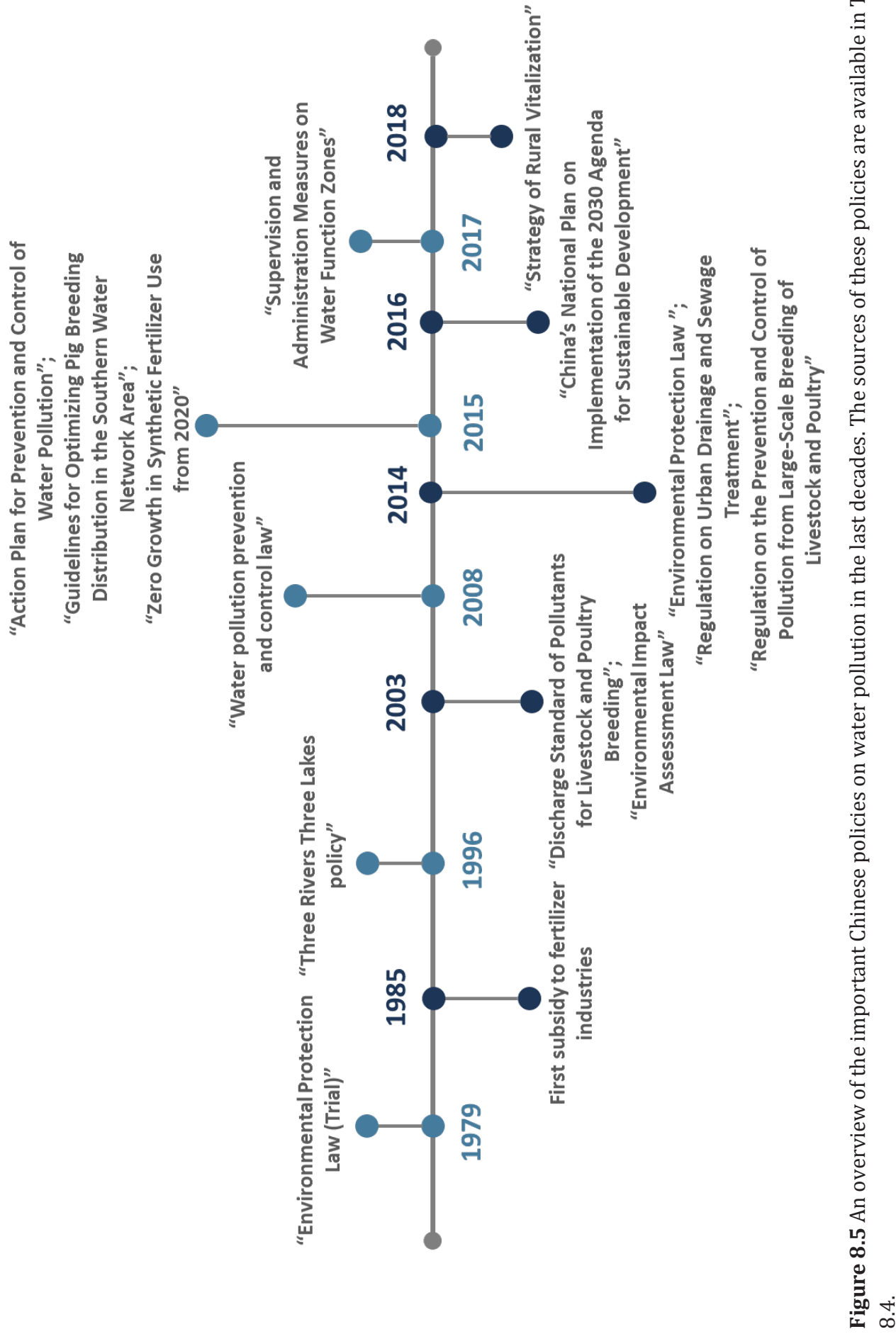




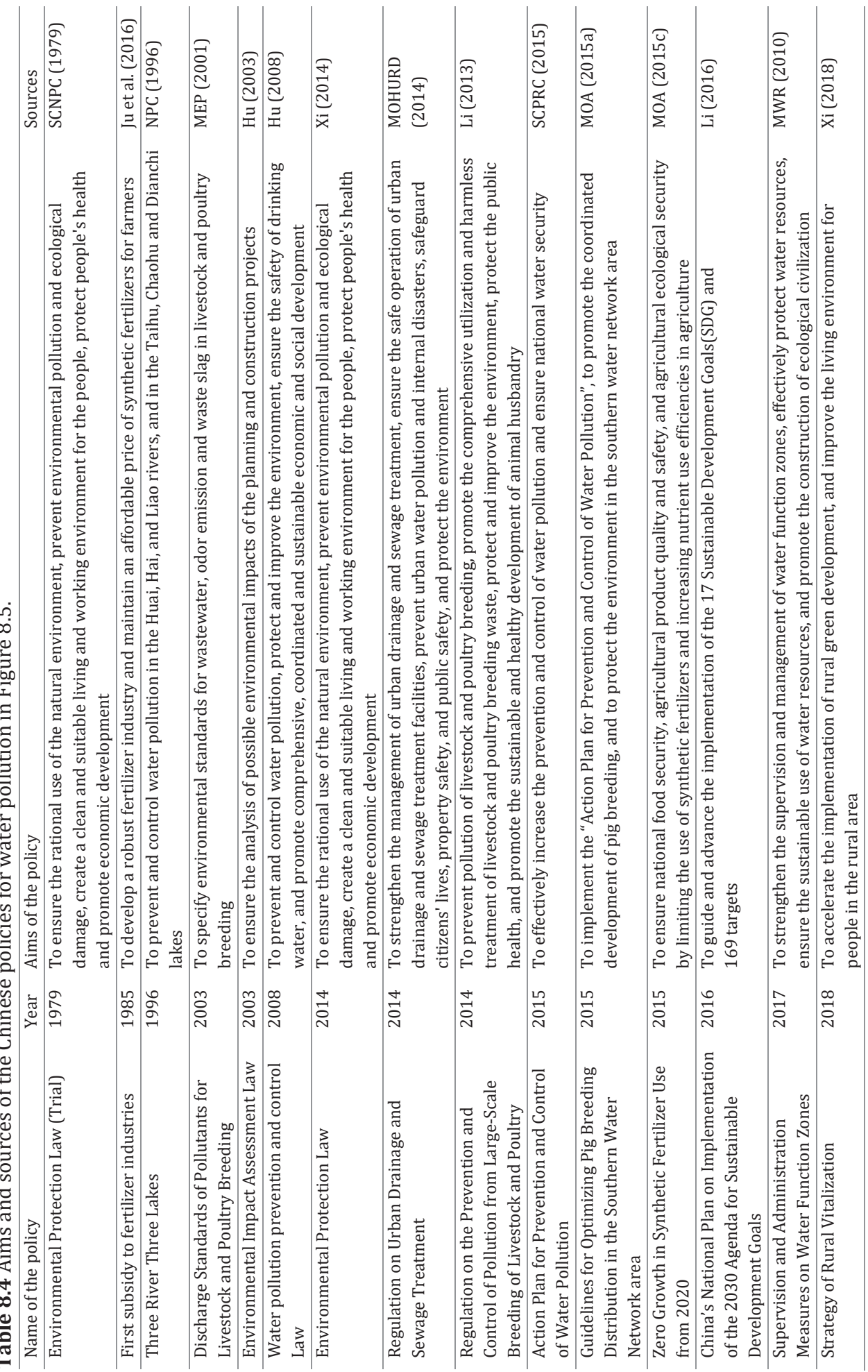




\subsubsection{Policy implications}

In recent decades, China has been developing fast in agriculture and urbanization at the expense of high resource use and environmental costs. Environmental concerns in China have been growing, especially since the 2000s. Several important environmental policies for prevention and control of water pollution were developed and implemented in recent years (Figure 8.5), focusing on manure management, fertilizer use, and sewage systems. The development of these policies confirmed two important messages of this thesis: 1) nutrient pollution in water systems has increased dramatically in China, and 2) direct discharge of animal manure and human waste are still the important sources of this nutrient pollution in the 2010s. Some examples of the policies are provided as follows.

In 2003 the "Discharge Standard of Pollutants for Livestock and Poultry Breeding" was introduced as the first guidance for manure management $(\mathrm{Hu}, 2003)$. This standard, however, was not always implemented by livestock farmers during the recent industrialization of livestock production. As the consequence, direct discharge of animal manure to rivers has become an important sources of water pollution in many regions in China, as shown in this thesis and several other NUFER or MARINA studies (Bai et al., 2014b; Ma et al., 2012b; Strokal et al., 2016a; Strokal et al., 2016b). To control water pollution by direct discharge of manure, the Chinese government implemented the "Regulation on the Prevention and Control of Pollution from Large-Scale Breeding of Livestock and Poultry" (Li, 2013) in 2014. Based on this regulation, several other policies such as the "Guidelines for Optimizing Pig Breeding Distribution in the Southern Water Network Area" were developed for better management of livestock production in regions where water quality is vulnerable to manure pollution (MOA, 2015a). In 2015, the "Zero Growth in Synthetic Fertilizer Use from 2020" was implemented to reduce the use of synthetic fertilizers by recycling animal manure in agriculture (MOA, 2015c). However, my research in this thesis shows that this policy is not effective in reducing future nutrient losses to water systems from food production in China because crops are still overfertilized (see Chapter 6).

Polices on sewage management were also developed as a response to water pollution caused by fast population growth and urbanization. One example is the "Regulation on Urban Drainage and Sewage Treatment" introduced in 2014 (MOHURD, 2014). Besides urban sewage, human waste in rural areas has been more important as a source of water 
pollution because of poor sewage management, shown in this thesis. Recently in 2018, the Chinese government introduced the "Strategy of Rural Vitalization" (Xi, 2018). With this policy, the government aims to accelerate the implementation of rural green (sustainable) development and improve the living environment for people in rural areas. Regulations on rural sewage treatment were included in this new policy.

In 2015, the general "Action Plan for Prevention and Control of Water Pollution" was developed and implemented in China (SCPRC, 2015). This action plan was used as the basis for the "China's National Plan on Implementation of the 2030 Agenda for Sustainable Development" in 2016 (Li, 2016). Follow up polices such as "Supervision and Administration Measures on Water Function Zones" were implemented in 2017 to monitor the progress of water pollution control in different water function zones (MWR, 2010). The action plan and its follow-up policies all have strong focuses on pollution management in agriculture and sewage systems, which are the important sources of nutrients in water systems shown by this thesis.

\subsubsection{Closing remarks}

My thesis is relevant for the development of future environmental policies for water pollution control in China. I provide a comprehensive understanding of nutrient pollution in water systems in China, with a focus on the challenges, trends, and solutions. I developed a new linked NUFER-MARINA model system. This linked model system simultaneously (1) provides quantitative information on nutrient pollution in water systems, and (2) improves the quantification of the spatial variabilities in the drivers and sources of nutrient pollution in water systems. In this thesis, I used quantitative indicators to assess water pollution in China. This makes it easier to understand and communicate modeling results with policymakers and stakeholders. My scenario analyses in Chapters 4-7 shows future nutrient pollution in water systems will likely increase under global change, and also provides potential options to reduce this pollution and to reach relevant SDGs. The key potential options for the future are improving nutrient efficiencies in agriculture (e.g., recycling of manure, reduce the use of synthetic fertilizers), efficient food production, and climate mitigation. Policymakers can take these options as the starting point to further develop feasible policies that complement the development of other sectors in society. Participatory approaches for developing future scenarios are highly recommended to consider different perspectives and uncertainties for policy 
implementation. All this hopefully will make a valuable contribution to the prevention and control of water pollution by nutrients and to the achievement of the Sustainable Development Goals in China. Moreover, my study serves as an example for other world regions. 


\section{References}

Alcamo J. Environmental futures: the practice of environmental scenario analysis. Vol 2: Elsevier, 2008.

Alcamo J., Van Vuuren D., Cramer W., Alder J., Bennett E., Carpenter S., Christensen V., Foley J., Maerker M., Schulze K. Changes in ecosystem services and their drivers across the scenarios. Ecosystems and human well-being: Scenarios 2005; 2: 297-373.

Alexandratos N., Bruinsma J. World agriculture towards 2030/2050: the 2012 revision. ESA Work. Pap 2012; 3.

Amin M.N., Kroeze C., Strokal M. Human waste: An underestimated source of nutrient pollution in coastal seas of Bangladesh, India and Pakistan. Marine Pollution Bulletin 2017; 118: 131140 .

Arnell N.W., Lloyd-Hughes B. The global-scale impacts of climate change on water resources and flooding under new climate and socio-economic scenarios. Climatic Change 2014; 122: 127-140.

Arnold J.G., Moriasi D.N., Gassman P.W., Abbaspour K.C., White M.J., Srinivasan R., Santhi C., Harmel R., Van Griensven A., Van Liew M.W. SWAT: Model use, calibration, and validation. Transactions of the ASABE 2012; 55: 1491-1508.

Augusiak J., Van den Brink P.J., Grimm V. Merging validation and evaluation of ecological models to 'evaludation': a review of terminology and a practical approach. Ecological Modelling 2014; 280: 117-128.

Bai X., Shi P., Liu Y. Society: Realizing China's urban dream. Nature News 2014a; 509: 158.

Bai Z., Jin S., Wu Y., zu Ermgassen E., Oenema O., Chadwick D., Lassaletta L., Velthof G.L., Zhao J., Ma L. China's pig relocation in balance. Nature Sustainability under revision.

Bai Z., Lu J., Zhao H., Velthof G.L., Oenema O., Chadwick D., Williams J.R., Jin S., Liu H., Wang M., Strokal M., Kroeze C., Hu C., Ma L. Designing Vulnerable Zones of Nitrogen and Phosphorus Transfers To Control Water Pollution in China. ACS Publications, 2018a.

Bai Z., Ma L., Ma W., Qin W., Velthof G.L., Oenema O., Zhang F. Changes in phosphorus use and losses in the food chain of China during 1950-2010 and forecasts for 2030. Nutrient cycling in agroecosystems 2016; 104: 361-372.

Bai Z., Ma L., Qin W., Chen Q., Oenema O., Zhang F. Changes in Pig Production in China and Their Effects on Nitrogen and Phosphorus Use and Losses. Environmental science \& technology 2014b; 48: 12742-12749.

Bai Z., Ma W., Ma L., Velthof G.L., Wei Z., Havlík P., Oenema O., Lee M.R., Zhang F. China's livestock transition: Driving forces, impacts, and consequences. Science advances 2018b; 4: eaar8534.

Bai Z.H., Ma L., Oenema O., Chen Q., Zhang F.S. Nitrogen and Phosphorus Use Efficiencies in Dairy Production in China. J. Environ. Qual. 2013; 42: 990-1001.

Basu N.B., Destouni G., Jawitz J.W., Thompson S.E., Loukinova N.V., Darracq A., Zanardo S., Yaeger M., Sivapalan M., Rinaldo A. Nutrient loads exported from managed catchments reveal emergent biogeochemical stationarity. Geophysical Research Letters 2010; 37.

Beusen A., Dekkers A., Bouwman A., Ludwig W., Harrison J. Estimation of global river transport of sediments and associated particulate C, N, and P. Global Biogeochemical Cycles 2005; 19. 
Beusen A.H., Bouwman A.F., Van Beek L.P., Mogollón J.M., Middelburg J.J. Global riverine N and P transport to ocean increased during the 20th century despite increased retention along the aquatic continuum. Biogeosciences 2016; 13: 2441-2451.

Billen G., Beusen A., Bouwman L., Garnier J. Anthropogenic nitrogen autotrophy and heterotrophy of the world's watersheds: Past, present, and future trends. Global Biogeochemical Cycles 2010; 24.

Billen G., Garnier J. River basin nutrient delivery to the coastal sea: assessing its potential to sustain new production of non-siliceous algae. Marine Chemistry 2007; 106: 148-160.

Billen G., Garnier J., Lassaletta L. The nitrogen cascade from agricultural soils to the sea: modelling nitrogen transfers at regional watershed and global scales. Philosophical Transactions of the Royal Society B: Biological Sciences 2013; 368: 20130123.

Börjeson L., Höjer M., Dreborg K.-H., Ekvall T., Finnveden G. Scenario types and techniques: towards a user's guide. Futures 2006; 38: 723-739.

Boudri J., Hordijk L., Kroeze C., Amann M., Cofala J., Bertok I., Junfeng L., Lin D., Shuang Z., Runquing $\mathrm{H}$. The potential contribution of renewable energy in air pollution abatement in China and India. Energy policy 2002; 30: 409-424.

Bouwman A., Beusen A., Billen G. Human alteration of the global nitrogen and phosphorus soil balances for the period 1970-2050. Global Biogeochemical Cycles 2009; 23: GB0A04.

Bouwman A., Van Drecht G., Van der Hoek K. Surface N balances and reactive N loss to the environment from global intensive agricultural production systems for the period 19702030. Science in China Series C: Life Sciences 2005; 48: 767-779.

Bouwman L., Goldewijk K.K., Van Der Hoek K.W., Beusen A.H., Van Vuuren D.P., Willems J., Rufino M.C., Stehfest E. Exploring global changes in nitrogen and phosphorus cycles in agriculture induced by livestock production over the 1900-2050 period. Proceedings of the National Academy of Sciences 2013; 110: 20882-20887.

Brink J.C. Modelling cost-effectiveness of interrelated emission reduction strategies. Wageningen Universiteit, Wageningen, 2003.

Burek P., Satoh Y., Fischer G., Kahil M., Scherzer A., Tramberend S., Nava L., Wada Y., Eisner S., Flörke M. Water Futures and Solution-Fast Track Initiative. 2016.

Burek P., Satoh Y., Greve P., Kahil T., Wada Y. The Community Water Model (CWATM)/Development of a community driven global water model. EGU General Assembly Conference Abstracts. 19, 2017, pp. 9769.

Burton C.H., Turner C. Manure management: Treatment strategies for sustainable agriculture: Editions Quae, 2003.

Cai C., Gu X., Ye Y., Yang C., Dai X., Chen D., Yang C. Assessment of pollutant loads discharged from aquaculture ponds around Taihu Lake, China. Aquaculture Research 2013; 44: 795-806.

Cai Z., Yan X. Understanding greenhouse gas emissions from croplands in China. ACS Symposium Series. 1072. American Chemical Society, 2011, pp. 91-120.

Cao L., Liu S., Ren J. Seasonal variations of particulate silicon in the Changjiang (Yangtze River) Estuary and its adjacent area. Acta Oceanologica Sinica 2013; 32: 1-10.

CAU. Farmers benefit from "Science and Technology Backyard" China Agricultural University, 2016, http://www1.cau.edu.cn/art/2015/7/7/art 8779 386961.html.

Chadwick D., Wei J., Yan'an T., Guanghui Y., Qirong S., Qing C. Improving manure nutrient management towards sustainable agricultural intensification in China. Agriculture, Ecosystems \& Environment 2015; 209: 34-46. 
Chai R., Niu Y., Huang L., Liu L., Wang H., Wu L., Zhang Y. Mitigation potential of greenhouse gases under different scenarios of optimal synthetic nitrogen application rate for grain crops in China. Nutrient cycling in agroecosystems 2013; 96: 15-28.

Chen M., Chen J., Sun F. Agricultural phosphorus flow and its environmental impacts in China. Science of the Total Environment 2008; 405: 140-152.

Chen W., Jia Y., Li E., Zhao S., Zhou Q., Liu L., Song L. Soil-based treatments of mechanically collected cyanobacterial blooms from Lake Taihu: efficiencies and potential risks. Environmental science \& technology 2012; 46: 13370-13376.

Chen X.-P., Cui Z.-L., Vitousek P.M., Cassman K.G., Matson P.A., Bai J.-S., Meng Q.-F., Hou P., Yue S.C., Römheld V. Integrated soil-crop system management for food security. Proceedings of the National Academy of Sciences 2011; 108: 6399-6404.

Chen X., Ma L., Ma W., Wu Z., Cui Z., Hou Y., Zhang F. What has caused the use of fertilizers to skyrocket in China? Nutrient Cycling in Agroecosystems 2018; 110: 241-255.

Chen X., Strokal M., Kroeze C., Ma L., Shen Z., Wu J., Chen X., Shi X. Seasonality in river export of nitrogen: A modelling approach for the Yangtze River. Science of the Total Environment 2019a; 671: 1282-1292.

Chen X., Strokal M., Van Vliet M.T., Stuiver J., Wang M., Bai Z., Ma L., Kroeze C. Multi-scale modeling of nutrient pollution in the rivers of China. Environmental science \& technology 2019b.

Chen Y., Fan C., Teubner K., Dokulil M. Changes of nutrients and phytoplankton chlorophyll-a in a large shallow lake, Taihu, China: an 8-year investigation. Hydrobiologia 2003; 506: 273279.

Chinese Nutrition Society. Dietary guidelines for Chinese residents (In Chinese). Beijing: People's medical publishing house, 2007.

Conway G.R., Pretty J.N. Unwelcome harvest: agriculture and pollution: Routledge, 2013.

Crespo Cuaresma J. Income projections for climate change research: A framework based on human capital dynamics. Global Environmental Change 2017; 42: 226-236.

Crippa M., Janssens-Maenhout G., Dentener F., Guizzardi D., Sindelarova K., Muntean M., Van Dingenen R., Granier C. Forty years of improvements in European air quality: regional policy-industry interactions with global impacts. Atmospheric Chemistry and Physics 2016; 16: 3825-3841.

Cuaresma J.C. Income projections for climate change research: A framework based on human capital dynamics. Global Environmental Change 2015; 42: 226-236.

Cui S., Shi Y., Groffman P.M., Schlesinger W.H., Zhu Y.-G. Centennial-scale analysis of the creation and fate of reactive nitrogen in China (1910-2010). Proceedings of the National Academy of Sciences 2013.

Cui S., Shi Y., Malik A., Lenzen M., Gao B., Huang W. A hybrid method for quantifying China's nitrogen footprint during urbanisation from 1990 to 2009. Environment International 2016; 97: 137-145.

Cui Z., Chen X., Zhang F. Current nitrogen management status and measures to improve the intensive wheat-maize system in China. Ambio 2010; 39: 376-384.

Dai Z., Du J., Zhang X., Su N., Li J. Variation of riverine material loads and environmental consequences on the Changjiang (Yangtze) Estuary in recent decades (1955- 2008). Environmental Science \& Technology 2010; 45: 223-227.

Dianwu Z., Anpu W. Estimation of anthropogenic ammonia emissions in asia. Atmospheric Environment 1994; 28: 689-694. 
Diaz R.J., Rosenberg R. Spreading dead zones and consequences for marine ecosystems. science 2008; 321: 926-929.

Döll P., Lehner B. Validation of a new global 30-min drainage direction map. Journal of Hydrology 2002; 258: 214-231.

Dong X.J., Wu Z.Y., Yan R.L., Guo C.M., Wu J., Ma L.J., JIa Y.L. Prevention and control of livestock and poultry pollution and environmental management. Environment and development 2018; 3: 67-70.

Du S., Mroz T.A., Zhai F., Popkin B.M. Rapid income growth adversely affects diet quality in Chinaparticularly for the poor! Social science \& medicine 2004; 59: 1505-1515.

Duan H., Ma R., Xu X., Kong F., Zhang S., Kong W., Hao J., Shang L. Two-decade reconstruction of algal blooms in China's Lake Taihu. Environmental Science \& Technology 2009; 43: 35223528.

Dumont E., Harrison J., Kroeze C., Bakker E., Seitzinger S. Global distribution and sources of dissolved inorganic nitrogen export to the coastal zone: Results from a spatially explicit, global model. Global Biogeochemical Cycles 2005; 19.

Edition F. Guidelines for drinking-water quality. WHO chronicle 2011; 38: 104-8.

Erisman J.W., Bleeker A., Hensen A., Vermeulen A. Agricultural air quality in Europe and the future perspectives. Atmospheric Environment 2008; 42: 3209-3217.

Ermolieva T., Winiwarter W., Fischer G., Cao G., Klimont Z., Schöpp W., Li Y., Asman W., Wagner F., Amann M. Integrated nitrogen management in China. IIASA Interim Report, IR-09-005 IIASA, Laxenburg, Austria 2009: p.59.

Fan M., Cui Z., Chen X., Jiang R., Zhang F. Integrated nutrient management for improving crop yields and nutrient utilization efficiencies in China. Journal of soil and water conservation 2008; 63: 126A-128A.

Fan M., Lu S., Jiang R., Liu X., Zhang F. Triangular transplanting pattern and split nitrogen fertilizer application increase rice yield and nitrogen fertilizer recovery. Agronomy journal 2009; 101: 1421-1425.

FAO. FAOSTAT Database Domains on Animal production Food and Agriculture Organization of the United Nations Statistics Division, 2015a, http://faostat3.fao.org/home/E

FAO. FAOSTAT Database Domains on World consumption in nutrients Food and Agriculture Organization of the United Nations Statistics Division, 2015b, http://faostat3.fao.org/home/E

FAO. FAOSTAT Database Domains on Inputs Food and Agriculture Organization of the United Nations Statistics Division, 2017a, http://faostat3.fao.org/home/E

FAO. FAOSTAT Database Domains on Population Food and Agriculture Organization of the United Nations Statistics Division, 2017b, http://faostat3.fao.org/home/E

FAO. FAOSTAT Database Domains on Trade Food and Agriculture Organization of the United Nations Statistics Division, 2019, http://faostat3.fao.org/home/E

Fekete B.M., Wisser D., Kroeze C., Mayorga E., Bouwman L., Wollheim W.M., Vörösmarty C. Millennium ecosystem assessment scenario drivers (1970-2050): climate and hydrological alterations. Global Biogeochemical Cycles 2010; 24.

Ferrier S., Ninan K.N., Leadley P., Alkemade R., Acosta L.A., Akçakaya H.R., Brotons L., Cheung W.W.L., Christensen V., Harhash K.A., Kabubo-Mariara J., Lundquist C., Obersteiner M., Pereira H.M., Peterson G., Pichs-Madruga R., Ravindranath N., Rondinini C., (eds.) B.A.W. The methodological assessment report on scenarios and models of biodiversity and 
ecosystem services. 348 pp. Secretariat of the Intergovernmental Science-Policy Platform on Biodiversity and Ecosystem Services, Bonn, Germany, 2016.

Fewtrell L., Bartram J. Water Quality: Guidelines, Standards \& Health: IWA publishing, 2001.

Fink G., Alcamo J., Flörke M., Reder K. Phosphorus loadings to the world's largest lakes: sources and trends. Global Biogeochemical Cycles 2018; 32: 617-634.

Fu B.-j., Zhuang X.-l., Jiang G.-b., Shi J.-b., Lu, Yi h. FEATURE: Environmental Problems and Challenges in China. Environmental Science \& Technology 2007; 41: 7597-7602.

Galloway J.N., Dentener F.J., Capone D.G., Boyer E.W., Howarth R.W., Seitzinger S.P., Asner G.P., Cleveland C., Green P., Holland E. Nitrogen cycles: past, present, and future. Biogeochemistry 2004; 70: 153-226.

Galloway J.N., Leach A.M., Erisman J.W., Bleeker A. Nitrogen: the historical progression from ignorance to knowledge, with a view to future solutions. Soil Research 2017; 55: 417-424.

Galloway J.N., Townsend A.R., Erisman J.W., Bekunda M., Cai Z., Freney J.R., Martinelli L.A., Seitzinger S.P., Sutton M.A. Transformation of the nitrogen cycle: recent trends, questions, and potential solutions. Science 2008; 320: 889-92.

Garnett T., Wilkes A. Appetite for Change. Social, Economic and Environmental Transformations in China's Food System 2014.

Garnier J., Beusen A., Thieu V., Billen G., Bouwman L. N: P: Si nutrient export ratios and ecological consequences in coastal seas evaluated by the ICEP approach. Global Biogeochemical Cycles 2010; 24.

Glibert P.M., Anderson D.M., Gentien P., Granéli E., Sellner K.G. The global, complex phenomena of harmful algal blooms. Oceanography. 18, 2005, pp. 136-147.

GOVUK. Using nitrogen fertilisers in nitrate vulnerable zones. 2018. Department for Environment, Food \& Rural Affairs and Environment Agency, 2015.

GPRC. China's National Plan on Implementation of the 2030 Agenda for Sustainable Development. In: China GotPsRo, editor, 2016.

Gu B., Ge Y., Chang S.X., Luo W., Chang J. Nitrate in groundwater of China: Sources and driving forces. Global Environmental Change 2013; 23: 1112-1121.

Gu B., Ju X., Chang J., Ge Y., Vitousek P.M. Integrated reactive nitrogen budgets and future trends in China. Proceedings of the National Academy of Sciences 2015; 112: 8792-8797.

Gu B.J., Ge Y., Ren Y., Xu B., Luo W.D., Jiang H., Gu B.H., Chang J. Atmospheric Reactive Nitrogen in China: Sources, Recent Trends, and Damage Costs. Environmental Science \& Technology 2012; 46: 9420-9427.

Guo J.H., Liu X.J., Zhang Y., Shen J.L., Han W.X., Zhang W.F., Christie P., Goulding K.W.T., Vitousek P.M., Zhang F.S. Significant Acidification in Major Chinese Croplands. Science 2010; 327: 1008-1010.

Guo L. Doing battle with the green monster of Taihu Lake. Science 2007; 317: 1166-1166.

Han H., Allan J.D., Scavia D. Influence of climate and human activities on the relationship between watershed nitrogen input and river export. Environmental Science \& Technology 2009; 43: 1916-1922.

Hartmann T.E., Yue S., Schulz R., He X., Chen X., Zhang F., Müller T. Yield and N use efficiency of a maize-wheat cropping system as affected by different fertilizer management strategies in a farmer's field of the North China Plain. Field Crops Research 2015; 174: 30-39. 
Heffer P., Gruère A., Roberts T. Assessment of Fertilizer Use by Crop at the Global Level. 2018. International Fertilizer Association (IFA) and International Plant Nutrition Institute (IPNI), 2017.

Hempel S., Frieler K., Warszawski L., Schewe J., Piontek F. A trend-preserving bias correction-the ISI-MIP approach. Earth System Dynamics 2013; 4: 219-236.

Hou Y., Ma L., Gao Z.L., Wang F.H., Sims J.T., Ma W.Q., Zhang F.S. The Driving Forces for Nitrogen and Phosphorus Flows in the Food Chain of China, 1980 to 2010. J. Environ. Qual. 2013; 42: 962-971.

Hou Y., Velthof G.L., Lesschen J.P., Staritsky I.G., Oenema O. Nutrient Recovery and Emissions of Ammonia, Nitrous Oxide, and Methane from Animal Manure in Europe: Effects of Manure Treatment Technologies. Environmental Science \& Technology 2017; 51: 375-383.

Hou Y., Velthof G.L., Oenema O. Mitigation of ammonia, nitrous oxide and methane emissions from manure management chains: a meta-analysis and integrated assessment. Global change biology 2015; 21: 1293-1312.

Howarth R., Swaney D., Boyer E., Marino R., Jaworski N., Goodale C. The influence of climate on average nitrogen export from large watersheds in the Northeastern United States. Nitrogen Cycling in the Americas: Natural and Anthropogenic Influences and Controls. Springer, 2006, pp. 163-186.

Howarth R.W., Boyer E.W., Pabich W.J., Galloway J.N. Nitrogen use in the United States from 19612000 and potential future trends. AMBIO: A Journal of the Human Environment 2002; 31: 88-96.

Hu J. Environmental Impact Assessment Law (In Chinese). In: Congress TSCotNPs, editor, Beijing, China, 2003.

Hu J. Environmental Protection Law (In Chinese). In: Congress TSCotNPs, editor, Beijing, China, 2008.

$\mathrm{Hu}$ J., Li S. Modeling the mass fluxes and transformations of nutrients in the Pearl River Delta, China. Journal of Marine Systems 2009; 78: 146-167.

Hu W., Zhai S., Zhu Z., Han H. Impacts of the Yangtze River water transfer on the restoration of Lake Taihu. Ecological Engineering 2008; 34: 30-49.

Huan Q., Pang R., Zhou Q., Leng K. Variation trends of nitrogen and phosphorus and the relationship with HABs in Shenzhen coastal waters (in Chinese). Marine Environmental Science 2016; 35.

Huang J., Arhonditsis G.B., Gao J., Kim D.-K., Dong F. Towards the development of a modeling framework to track nitrogen export from lowland artificial watersheds (polders). Water research 2018; 133: 319-337.

Huang J., Gao J., Jiang Y., Yin H., Amiri B.J. Sources, distribution and export coefficient of phosphorus in lowland polders of Lake Taihu Basin, China. Environmental Pollution 2017; 231: $1274-1283$.

Huang J., Huang Z., Jia X., Hu R., Xiang C. Long-term reduction of nitrogen fertilizer use through knowledge training in rice production in China. Agricultural Systems 2015; 135: 105-111.

Huang X., Song Y., Li M., Li J., Huo Q., Cai X., Zhu T., Hu M., Zhang H. A high-resolution ammonia emission inventory in China. Global Biogeochemical Cycles 2012; 26.

IAEG U. Final list of proposed Sustainable Development Goal indicators. Report of the InterAgency and Expert Group on Sustainable Development Goal Indicators (E/CN. 3/2016/2/Rev. 1), 2016.

IEA. World Energy Outlook 2007: Focus on China and India. International Energy Agency, 2007. 
IIASA. SSP Database (Shared Socioeconomic Pathways) - Version 1.0, 2013, https://tntcat.iiasa.ac.at/SspDb/dsd?Action=htmlpage\&page=about.

Janse J.H., Domis L.N.D.S., Scheffer M., Lijklema L., Van Liere L., Klinge M., Mooij W.M. Critical phosphorus loading of different types of shallow lakes and the consequences for management estimated with the ecosystem model PCLake. Limnologica-Ecology and Management of Inland Waters 2008; 38: 203-219.

Janssen A.B.G., de Jager V.C.L., Janse J.H., Kong X., Liu S., Ye Q., Mooij W.M. Spatial identification of critical nutrient loads of large shallow lakes: Implications for Lake Taihu (China). Water Research 2017; 119: 276-287.

Janssen A.B.G., Teurlincx S., An S., Janse J.H., Paerl H.W., Mooij W.M. Alternative stable states in large shallow lakes? Journal of Great Lakes Research 2014; 40: 813-826.

Jia W. Studies on the Evaluation of Nutrient Resources Derived from Manrue and Optimized Utilization in Areble Land of China (In Chinese with English abstract). Resource and Environmental Sciences. Ph.D. China Agriculture University, 2014, pp. 137.

Jiang L., O'Neill B.C. Global urbanization projections for the Shared Socioeconomic Pathways. Global Environmental Change 2017; 42: 193-199.

Jin X., Xu Q., Huang C. Current status and future tendency of lake eutrophication in China. Science in China Series C: Life Sciences 2005; 48: 948-954.

Ju X.-T., Xing G.-X., Chen X.-P., Zhang S.-L., Zhang L.-J., Liu X.-J., Cui Z.-L., Yin B., Christie P., Zhu Z.-L. Reducing environmental risk by improving $\mathrm{N}$ management in intensive Chinese agricultural systems. Proceedings of the National Academy of Sciences 2009; 106: 30413046.

Ju X., Gu B., Wu Y., Galloway J.N. Reducing China's fertilizer use by increasing farm size. Global environmental change 2016; 41: 26-32.

Ju X., Zhang F., Bao X., Römheld V., Roelcke M. Utilization and management of organic wastes in Chinese agriculture: past, present and perspectives. Science in China Series C: Life Sciences 2005; 48: 965-979.

Kang Y., Liu M., Song Y., Huang X., Yao H., Cai X., Zhang H., Kang L., Liu X., Yan X. High-resolution ammonia emissions inventories in China from 1980 to 2012. Atmospheric Chemistry and Physics 2016; 16: 2043-2058.

Kearney J. Food consumption trends and drivers. Philosophical transactions of the royal society B: biological sciences 2010; 365: 2793-2807.

Khiewwijit R. New wastewater treatment concepts towards energy saving and resource recovery: Wageningen University, 2016.

Kok K. Multi-scale integration and synthesis of scenarios and adaptation narratives. 2016.

Kong X., He Q., Yang B., He W., Xu F., Janssen A.B., Kuiper J.J., Van Gerven L.P., Qin N., Jiang Y. Hydrological regulation drives regime shifts: evidence from paleolimnology and ecosystem modeling of a large shallow Chinese lake. Global change biology 2017; 23: 737 754.

Kristensen P. The DPSIR framework. National Environmental Research Institute, Denmark 2004; 10.

Kroeze C., Bouwman L., Seitzinger S. Modeling global nutrient export from watersheds. Current Opinion in Environmental Sustainability 2012; 4: 195-202.

Kurokawa J., Ohara T., Morikawa T., Hanayama S., Janssens-Maenhout G., Fukui T., Kawashima K., Akimoto H. Emissions of air pollutants and greenhouse gases over Asian regions during 
2000-2008: Regional Emission inventory in ASia (REAS) version 2. Atmospheric Chemistry and Physics 2013; 13: 11019-11058.

Lai G., Yu G., Gui F. Preliminary study on assessment of nutrient transport in the Taihu Basin based on SWAT modeling. Science in China Series D 2006; 49: 135-145.

Larson C. Losing arable land, China faces stark choice: adapt or go hungry. American Association for the Advancement of Science, 2013.

Lassaletta L., Billen G., Garnier J., Bouwman L., Velazquez E., Mueller N.D., Gerber J.S. Nitrogen use in the global food system: past trends and future trajectories of agronomic performance, pollution, trade, and dietary demand. Environmental Research Letters 2016; 11: 095007.

Le C., Zha Y., Li Y., Sun D., Lu H., Yin B. Eutrophication of lake waters in China: cost, causes, and control. Environmental Management 2010; 45: 662-668.

Lee J.-W., Hong S.-Y., Chang E.-C., Suh M.-S., Kang H.-S. Assessment of future climate change over East Asia due to the RCP scenarios downscaled by GRIMs-RMP. Climate Dynamics 2014; 42: 733-747.

Li A., Strokal M., Bai Z., Kroeze C., Ma L. How to avoid coastal eutrophication-a back-casting study for the North China Plain. Science of The Total Environment 2019a.

Li D., Wu N., Tang S., Su G., Li X., Zhang Y., Wang G., Zhang J., Liu H., Hecker M. Factors associated with blooms of cyanobacteria in a large shallow lake, China. Environmental Sciences Europe 2018; 30: 27.

Li G., Huang G., Li H., van Ittersum M., Leffelaar P., Zhang F. Identifying potential strategies in the key sectors of China's food chain to implement sustainable phosphorus management: a review. Nutrient Cycling in Agroecosystems 2015a: 1-19.

Li H.-M., Tang H.-J., Shi X.-Y., Zhang C.-S., Wang X.-L. Increased nutrient loads from the Changjiang (Yangtze) River have led to increased Harmful Algal Blooms. Harmful Algae 2014a; 39: 92101.

Li H., Liu J., Li G., Shen J., Bergström L., Zhang F. Past, present, and future use of phosphorus in Chinese agriculture and its influence on phosphorus losses. Ambio 2015b; 44: 274-285.

Li K. The Regulation on the Prevention and Control of Pollution from Large-scale Breeding of Livestock and Poultry (In Chinese). In: China OotSCotPsRo, editor, Beijing, China, 2013.

Li K. China's National Plan on Implementation of the 2030 Agenda for Sustainable Development Goals (In Chinese). In: China OotSCotPsRo, editor, Beijing, China, 2016.

Li X., Hu C., Delgado J.A., Zhang Y., Ouyang Z. Increased nitrogen use efficiencies as a key mitigation alternative to reduce nitrate leaching in north china plain. Agricultural Water Management 2007; 89: 137-147.

Li X., Janssen A.B., de Klein J.J., Kroeze C., Strokal M., Ma L., Zheng Y. Modeling nutrients in Lake Dianchi (China) and its watershed. Agricultural Water Management 2019b; 212: 48-59.

Li X., Yang L., Yan W. Model analysis of dissolved inorganic phosphorus exports from the Yangtze river to the estuary. Nutrient cycling in Agroecosystems 2011a; 90: 157-170.

Li Y., Acharya K., Stone M.C., Yu Z., Young M.H., Shafer D.S., Zhu J., Gray K., Stone A., Fan L. Spatiotemporal patterns in nutrient loads, nutrient concentrations, and algal biomass in Lake Taihu, China. Lake and reservoir management 2011b; 27: 298-309.

Li Y., Acharya K., Yu Z. Modeling impacts of Yangtze River water transfer on water ages in Lake Taihu, China. Ecological Engineering 2011c; 37: 325-334. 
Li Y., Tang C., Wang C., Anim D.O., Yu Z., Acharya K. Improved Yangtze River Diversions: Are they helping to solve algal bloom problems in Lake Taihu, China? Ecological Engineering 2013; 51: 104-116.

Li Y., Zhang W., Ma L., Wu L., Shen J., Davies W.J., Oenema O., Zhang F., Dou Z. An analysis of C hina's grain production: looking back and looking forward. Food and Energy Security 2014b; 3: 19-32.

Liang X., Lettenmaier D.P., Wood E.F., Burges S.J. A simple hydrologically based model of land surface water and energy fluxes for general circulation models. Journal of Geophysical Research: Atmospheres 1994; 99: 14415-14428.

Liu B., Liu H., Zhang B., Bi J. Modeling nutrient release in the Tai Lake Basin of China: source identification and policy implications. Environmental management 2013a; 51: 724-737.

Liu C., Watanabe M., Wang Q. Changes in nitrogen budgets and nitrogen use efficiency in the agroecosystems of the Changjiang River basin between 1980 and 2000. Nutrient cycling in agroecosystems 2008; 80: 19-37.

Liu S., Hong G.-H., Zhang J., Ye X., Jiang X. Nutrient budgets for large Chinese estuaries. Biogeosciences 2009; 6: 2245-2263.

Liu S., Xie Z., Zeng Y., Liu B., Li R., Wang Y., Wang L., Qin P., Jia B., Xie J. Effects of anthropogenic nitrogen discharge on dissolved inorganic nitrogen transport in global rivers. Global change biology 2019; 25: 1493-1513.

Liu S.M., Li L.W., Zhang G.L., Liu Z., Yu Z., Ren J.L. Impacts of human activities on nutrient transports in the Huanghe (Yellow River) estuary. Journal of Hydrology 2012; 430: 103-110.

Liu X., Beusen A.H., Van Beek L.P., Mogollón J.M., Ran X., Bouwman A.F. Exploring spatiotemporal changes of the Yangtze River (Changjiang) nitrogen and phosphorus sources, retention and export to the East China Sea and Yellow Sea. Water research 2018a.

Liu X., Duan L., Mo J., Du E., Shen J., Lu X., Zhang Y., Zhou X., He C., Zhang F. Nitrogen deposition and its ecological impact in China: an overview. Environmental pollution 2011; 159: 22512264.

Liu X., Ju X., Zhang F., Pan J., Christie P. Nitrogen dynamics and budgets in a winter wheat-maize cropping system in the North China Plain. Field Crops Research 2003; 83: 111-124.

Liu X., Sheng H., Jiang S., Yuan Z., Zhang C., Elser J.J. Intensification of phosphorus cycling in China since the 1600s. Proceedings of the National Academy of Sciences 2016; 113: 2609-2614.

Liu X.J., Zhang Y., Han W.X., Tang A.H., Shen J.L., Cui Z.L., Vitousek P., Erisman J.W., Goulding K., Christie P., Fangmeier A., Zhang F.S. Enhanced nitrogen deposition over China. Nature 2013b; 494: 459-462.

Liu X.N., Li Y.P., Zhu J.N., Zhang B.S., Pang Z.P., Ji Z.S. Dung Treatment and Recycling Mode in Largescale Dairy Farm (In Chinese with English abstract). Chinese Agricultural Science Bulletin 2018b; 34: 129-133.

Liu Y., Mol A.P.J., Chen J. Material Flow and Ecological Restructuring in China. Journal of Industrial Ecology 2004; 8: 103-120.

Loyon L., Burton C., Misselbrook T., Webb J., Philippe F., Aguilar M., Doreau M., Hassouna M., Veldkamp T., Dourmad J. Best available technology for European livestock farms: Availability, effectiveness and uptake. Journal of environmental management 2016; 166: $1-11$.

Lu D., Lu F., Pan J., Cui Z., Zou C., Chen X., He M., Wang Z. The effects of cultivar and nitrogen management on wheat yield and nitrogen use efficiency in the North China Plain. Field Crops Research 2015; 171: 157-164. 
Luo J., Yin J., Cai L., Zhang K., Hyde K.D. Freshwater fungi in Lake Dianchi, a heavily polluted lake in Yunnan, China. Fungal Diversity 2004.

Ma D.C., Hu S.Y., Chen D.J., Li Y.R. Substance flow analysis as a tool for the elucidation of anthropogenic phosphorus metabolism in China. Journal of Cleaner Production 2012a; 2930: 188-198.

Ma L., Bai Z., Ma W., Guo M., Jiang R., Liu J., Oenema O., Velthof G.L., Whitmore A.P., Crawford J., Dobermann A., Schwoob M., Zhang F. Exploring Future Food Provision Scenarios for China. Environmental Science \& Technology 2019; 53: 1385-1393.

Ma L., Feng S., Reidsma P., Qu F., Heerink N. Identifying entry points to improve fertilizer use efficiency in Taihu Basin, China. Land Use Policy 2014a; 37: 52-59.

Ma L., Guo J., Velthof G.L., Li Y., Chen Q., Ma W., Oenema O., Zhang F. Impacts of urban expansion on nitrogen and phosphorus flows in the food system of Beijing from 1978 to 2008. Global environmental change 2014b; 28: 192-204.

Ma L., Ma W., Velthof G., Wang F., Qin W., Zhang F., Oenema O. Modeling nutrient flows in the food chain of China. Journal of environmental quality 2010; 39: 1279-1289.

Ma L., Velthof G., Wang F., Qin W., Zhang W., Liu Z., Zhang Y., Wei J., Lesschen J., Ma W. Nitrogen and phosphorus use efficiencies and losses in the food chain in China at regional scales in 1980 and 2005. Science of the Total Environment 2012b; 434: 51-61.

Ma L., Wang F., Zhang W., Ma W., Velthof G., Qin W., Oenema O., Zhang F. Environmental assessment of management options for nutrient flows in the food chain in China. Environmental science \& technology 2013a; 47: 7260-7268.

Ma L., Zhang W.F., Ma W.Q., Velthof G.L., Oenema O., Zhang F.S. An Analysis of Developments and Challenges in Nutrient Management in China. Jounal of Environmental Quality 2013b; 42: 951-961.

Ma W., Li J., Ma L., Wang F., Sisák I., Cushman G., Zhang F. Nitrogen flow and use efficiency in production and utilization of wheat, rice, and maize in China. Agricultural Systems 2008; 99: 53-63.

Maier H.R., Guillaume J.H., van Delden H., Riddell G.A., Haasnoot M., Kwakkel J.H. An uncertain future, deep uncertainty, scenarios, robustness and adaptation: How do they fit together? Environmental Modelling \& Software 2016; 81: 154-164.

Mayorga E., Seitzinger S.P., Harrison J.A., Dumont E., Beusen A.H., Bouwman A., Fekete B.M., Kroeze C., Van Drecht G. Global nutrient export from WaterSheds 2 (NEWS 2): model development and implementation. Environmental Modelling \& Software 2010; 25: 837-853.

McCrackin M.L., Harrison J.A., Compton J.E. Factors influencing export of dissolved inorganic nitrogen by major rivers: A new, seasonal, spatially explicit, global model. Global biogeochemical cycles 2014; 28: 269-285.

McCrackin M.L., Harrison J.A., Compton J.E. Future riverine nitrogen export to coastal regions in the United States: Prospects for improving water quality. Journal of environmental quality 2015; 44: 345-355.

Meng Q., Sun Q., Chen X., Cui Z., Yue S., Zhang F., Römheld V. Alternative cropping systems for sustainable water and nitrogen use in the North China Plain. Agriculture, Ecosystems \& Environment 2012; 146: 93-102.

Meng X.-m., Zhang H.-w., Sun T., Wang Y., Zhong D.-s., Li Y.-s., Wang Y.-k. Application of Gini Coefficient in Total Pollutant Load Allocation for Surface Water [J]. China Water \& Wastewater 2008; 23. 
MEP. Discharge Standard of Pollutants for Livestock and Poultry Breeding (in Chinese). 2015. Ministry of Environemtnal Protection of the People's Republic of China, 2001.

MEP. Environmental Quality Standard for Surface Water. In: China MoEaEotPsRo, editor. GB 38382002, Beijing, China, 2002.

MEP. Environment Quality Bulletin, China, 2012. In: Ministry of Environmental Protection C, editor. Ministry of Environmental Protection, China, 2012.

MEP. Environment Quality Bulletin, China, 2013. In: Ministry of Environmental Protection C, editor. Ministry of Environmental Protection, China, 2013a.

MEP. National list of operating centralized wastewater treatment plants. In: China. MoEaEotPsRo, editor. Ministry of Ecology and Environment of the People's Republic of China., 2013b.

MOA. The Chinese agricultural statistical report. (In Chinese). Ministry of Agriculture. China Agricultural Press, Beijing, China, 2011.

MOA. The Chinese agricultural statistical report. (In Chinese). Ministry of Agriculture. China Agricultural Press, Beijing, China, 2014.

MOA. Guidelines for Optimizing Pig Breeding Distribution in the Southern Water Network area (in Chinese). 2016. Ministry of Agriculture of the People's Republic of China, 2015a.

MOA. Plan of actions aiming for zero growth in synthetic fertilizer use from 2020 onwards (in Chinese). 2015. Ministry of Agriculture of the People's Republic of China, 2015b.

MOA. Zero growth in synthetic fertilizer use from 2020 onwards (in Chinese). 2015. Ministry of Agriculture of the People's Republic of China, 2015c.

MOA. Livestock and poultry manure utilization Action Program (2017- 2020). In: China MoAaRAoTPsRo, editor, Beijing, China, 2017.

MOHURD. China Urban-Rural Construction Statistical Yearbook 2001. In: China. MoHaURDotPsRo, editor. Ministry of Housing and Urban-Rural Development of the People's Republic of China., 2001.

MOHURD. Bulletin for Drainage and Sewage Treatment Status in China, 2006-2010 (in Chinese). In: China. MoHaU-RDotPsRo, editor. Ministry of Housing and Urban-Rural Development of the People's Republic of China., 2012.

MOHURD. Regulation on Urban Drainage and Sewage Treatment (In Chinese). In: China. MoHaURDotPsRo, editor. Ministry of Housing and Urban-Rural Development of the People's Republic of China., 2014.

Morée A., Beusen A., Bouwman A., Willems W. Exploring global nitrogen and phosphorus flows in urban wastes during the twentieth century. Global Biogeochemical Cycles 2013; 27: 836846.

Moriasi D.N., Arnold J.G., Van Liew M.W., Bingner R.L., Harmel R.D., Veith T.L. Model evaluation guidelines for systematic quantification of accuracy in watershed simulations. Transactions of the ASABE 2007; 50: 885-900.

Mosier A., Syers J.K., Freney J.R. Agriculture and the nitrogen cycle: assessing the impacts of fertilizer use on food production and the environment. Vol 65: Island Press, 2013.

Müller B., Berg M., Yao Z.P., Zhang X.F., Wang D., Pfluger A. How polluted is the Yangtze river? Water quality downstream from the Three Gorges Dam. Science of the total environment 2008; 402: 232-247.

MWR. Water Resources Bulletin of China in 2010 (in Chinese). In: Resources MoW, editor. China Water Resources \& Hydropower Press, Beijing, China, 2010. 
MWR. Water Resources Bulletin of China in 2013 (in Chinese). In: China. MoWRotPsRo, editor. Ministry of Water Resources of the People's Republic of China., 2013.

Nakicenovic N., Alcamo J., Grubler A., Riahi K., Roehrl R., Rogner H.-H., Victor N. Special report on emissions scenarios (SRES), a special report of Working Group III of the intergovernmental panel on climate change: Cambridge University Press, 2000.

Nakicenovic N., Lempert R.J., Janetos A.C. A framework for the development of new socioeconomic scenarios for climate change research: introductory essay. Climatic Change 2014; 122: 351-361.

Nakicenovic N., Swart R. Special report on emissions scenarios. Special Report on Emissions Scenarios, Edited by Nebojsa Nakicenovic and Robert Swart, pp. 612. ISBN 0521804930. Cambridge, UK: Cambridge University Press, July 2000. 2000; 1.

Nasir I.M., Mohd Ghazi T.I., Omar R. Anaerobic digestion technology in livestock manure treatment for biogas production: a review. Engineering in Life Sciences 2012; 12: 258-269.

Nayak D., Cheng K., Wang W., Koslowski F., Yan X., Guo M., Newbold J., Moran D., Cardenas L., Pan G. Technical options to reduce greenhouse gas emissions from croplands and grasslands in China. 2013.

NBSC. China statistical yearbook. (In Chinese). National Bureau of Statistics of China. China Statistic Press, Beijing, China, 2011.

NBSC. China statistical yearbook. (In Chinese). National Bureau of Statistics of China. China Statistic Press, Beijing, China, 2013.

NBSC. China statistical yearbook. (In Chinese). National Bureau of Statistics of China. China Statistic Press, Beijing, China, 2014.

NDRC-MOHURD. National Urban Sewage Treatment and Recycling Planning in 13th Five-Year (2015-2020). In: National Development and Reform Commission MoHaU-RDotPsRoC, editor, 2016.

Nhamo G., Nhemachena C., Nhamo S. Is 2030 too soon for Africa to achieve the water and sanitation sustainable development goal? Science of The Total Environment 2019; 669: 129-139.

NPC. The ninth Five-Year Plan (In Chinese). In: Congress TNPs, editor, Beijing, China, 1996.

O’Neill B.C., Kriegler E., Ebi K.L., Kemp-Benedict E., Riahi K., Rothman D.S., van Ruijven B.J., van Vuuren D.P., Birkmann J., Kok K., Levy M., Solecki W. The roads ahead: Narratives for shared socioeconomic pathways describing world futures in the 21st century. Global Environmental Change 2017; 42: 169-180.

O’Neill B.C., Kriegler E., Riahi K., Ebi K.L., Hallegatte S., Carter T.R., Mathur R., van Vuuren D.P. A new scenario framework for climate change research: the concept of shared socioeconomic pathways. Climatic Change 2014; 122: 387-400.

Oenema 0., Tamminga S. Nitrogen in global animal production and management options for improving nitrogen use efficiency. Science in China Series C: Life Sciences 2005; 48: 871887.

Oenema O., Witzke H., Klimont Z., Lesschen J., Velthof G. Integrated assessment of promising measures to decrease nitrogen losses from agriculture in EU-27. Agriculture, ecosystems \& environment 2009; 133: 280-288.

Paerl H.W., Huisman J. Climate change: a catalyst for global expansion of harmful cyanobacterial blooms. Environmental microbiology reports 2009; 1:27-37. 
Pan G., Krom M.D., Zhang M., Zhang X., Wang L., Dai L., Sheng Y., Mortimer R.J. Impact of suspended inorganic particles on phosphorus cycling in the Yellow River (China). Environmental science \& technology 2013; 47: 9685-9692.

Pedde S., Kroeze C., Mayorga E., Seitzinger S.P. Modeling sources of nutrients in rivers draining into the Bay of Bengal-a scenario analysis. Regional Environmental Change 2017; 17: 2495-2506.

Popp A., Calvin K., Fujimori S., Havlik P., Humpenöder F., Stehfest E., Bodirsky B.L., Dietrich J.P., Doelmann J.C., Gusti M., Hasegawa T., Kyle P., Obersteiner M., Tabeau A., Takahashi K., Valin H., Waldhoff S., Weindl I., Wise M., Kriegler E., Lotze-Campen H., Fricko O., Riahi K., Vuuren D.P.v. Land-use futures in the shared socio-economic pathways. Global Environmental Change 2017; 42: 331-345.

Qin B., Liu Z., Havens K. Eutrophication of shallow lakes with special reference to Lake Taihu, China. Vol 194: Springer Science \& Business Media, 2007a.

Qin B., Xu P., Wu Q., Luo L., Zhang Y. Environmental issues of lake Taihu, China. Eutrophication of Shallow Lakes with Special Reference to Lake Taihu, China. Springer, 2007b, pp. 3-14.

Qin B., Zhu G., Gao G., Zhang Y., Li W., Paerl H.W., Carmichael W.W. A drinking water crisis in Lake Taihu, China: linkage to climatic variability and lake management. Environmental Management 2010; 45: 105-112.

Qin W. Exploring options for improving water and nitrogen use efficiency in crop production systems: Wageningen University, 2015.

Qin W., Ma L., Zhang F., Oenema O. Urbanization affects water and nitrogen use efficiencies in food chain. Agro Environ 20122012.

Qu H.J., Kroeze C. Past and future trends in nutrients export by rivers to the coastal waters of China. Science of the Total Environment 2010; 408: 2075-2086.

Qu H.J., Kroeze C. Nutrient export by rivers to the coastal waters of China: management strategies and future trends. Regional Environmental Change 2012; 12: 153-167.

Rabalais N.N., Turner R.E., Díaz R.J., Justić D. Global change and eutrophication of coastal waters. ICES Journal of Marine Science 2009; 66: 1528-1537.

Rao S., Klimont Z., Smith S.J., Van Dingenen R., Dentener F., Bouwman L., Riahi K., Amann M., Bodirsky B.L., van Vuuren D.P. Future air pollution in the Shared Socio-economic Pathways. Global Environmental Change 2017; 42: 346-358.

Reay D.S., Davidson E.A., Smith K.A., Smith P., Melillo J.M., Dentener F., Crutzen P.J. Global agriculture and nitrous oxide emissions. Nature Climate Change 2012; 2: 410-416.

Reidsma P., Feng S., van Loon M., Luo X., Kang C., Lubbers M., Kanellopoulos A., Wolf J., van Ittersum M.K., Qu F. Integrated assessment of agricultural land use policies on nutrient pollution and sustainable development in Taihu Basin, China. Environmental science \& policy 2012; 18: 66-76.

RESDC. 2016. Data Center for Resources and Environmental Sciences Chinese Academy of Sciences 2013.

Rosenzweig C., Jones J., Hatfield J., Ruane A., Boote K., Thorburn P., Antle J., Nelson G., Porter C., Janssen S. The agricultural model intercomparison and improvement project (AgMIP): protocols and pilot studies. Agricultural and Forest Meteorology 2013; 170: 166-182.

Scanes C. The global importance of poultry. Poultry science 2007; 86: 1057-1058.

Schneider M. Feeding China's Pigs: Implications for the Environment, China's Smallholder Farmers and Food Security. Institute for Agriculture and Trade Policy, 2011. 
Schöpp W., Amann M., Cofala J., Heyes C., Klimont Z. Integrated assessment of European air pollution emission control strategies. Environmental Modelling \& Software 1998; 14: 1-9.

SCNPC. Environmental Protection Law (Trial) (In Chinese). In: Congress TSCotNPs, editor, Beijing, China, 1979.

SCPRC. Action Plan for Prevention and Control of Water Pollution (In Chinese). In: China TSCtPsRo, editor, Beijing, China, 2015.

Seitzinger S., Harrison J., Dumont E., Beusen A.H., Bouwman A. Sources and delivery of carbon, nitrogen, and phosphorus to the coastal zone: An overview of Global Nutrient Export from Watersheds (NEWS) models and their application. Global Biogeochemical Cycles 2005; 19.

Seitzinger S., Mayorga E., Bouwman A., Kroeze C., Beusen A., Billen G., Van Drecht G., Dumont E., Fekete B., Garnier J. Global river nutrient export: A scenario analysis of past and future trends. Global Biogeochemical Cycles 2010; 24.

Sharpley A., Jarvie H.P., Buda A., May L., Spears B., Kleinman P. Phosphorus legacy: overcoming the effects of past management practices to mitigate future water quality impairment. Journal of environmental quality $2013 ; 42$ : 1308-1326.

Sims J., Ma L., Oenema O., Dou Z., Zhang F. Advances and challenges for nutrient management in China in the 21st century. Journal of environmental quality 2013; 42: 947-950.

Sinha E., Michalak A., Balaji V. Eutrophication will increase during the 21st century as a result of precipitation changes. Science 2017; 357: 405-408.

Sinha E., Michalak A., Calvin K., Lawrence P. Societal decisions about climate mitigation will have dramatic impacts on eutrophication in the 21 st century. Nature communications 2019; 10: 939.

SOA. Chinese Marine Environmental Bulletin. 2012. China's State Oceanic Administration, 2012.

Stiftung B. Sustainable Development Solutions Network. SDG Index and Dashboards Report 2018. Global responsibilities: implementing the goals. , 2018.

Streets D.G., Bond T., Carmichael G., Fernandes S., Fu Q., He D., Klimont Z., Nelson S., Tsai N., Wang M.Q. An inventory of gaseous and primary aerosol emissions in Asia in the year 2000. Journal of Geophysical Research: Atmospheres 2003; 108.

Strokal M. River export of nutrients to the coastal waters of China: the MARINA model to assess sources, effects and solutions. 2016.

Strokal M., de Vries W. Dynamic modelling of phosphorus export at river basin scale based on Global NEWS. Alterra Wageningen UR, Wageningen, the Netherlands, 2012, pp. 100.

Strokal M., Kroeze C. Nitrogen and phosphorus inputs to the Black Sea in 1970-2050. 2013; 13: 179-192.

Strokal M., Kroeze C., Li L., Luan S., Wang H., Yang S., Zhang Y. Modelling nitrogen and phosphorus export by the Pearl River in China 1970-2050. Water Pollution XII 2014a; 182: 309.

Strokal M., Kroeze C., Li L., Luan S., Wang H., Yang S., Zhang Y. Increasing dissolved nitrogen and phosphorus export by the Pearl River (Zhujiang): a modeling approach at the sub-basin scale to assess effective nutrient management. Biogeochemistry 2015; 125: 221-242.

Strokal M., Kroeze C., Wang M., Bai Z., Ma L. The MARINA model (Model to Assess River Inputs of Nutrients to seAs): Model description and results for China. Science of The Total Environment 2016a; 562: 869-888.

Strokal M., Kroeze C., Wang M., Ma L. Reducing future river export of nutrients to coastal waters of China in optimistic scenarios. Science of The Total Environment 2017; 579: 517-528. 
Strokal M., Ma L., Bai Z., Luan S., Kroeze C., Oenema O., Velthof G., Zhang F. Alarming nutrient pollution of Chinese rivers as a result of agricultural transitions. Environmental Research Letters 2016b; 11: 024014.

Strokal M., Spanier J.E., Kroeze C., Koelmans A.A., Flörke M., Franssen W., Hofstra N., Langan S., Tang T., Van Vliet M.T.H., Wada Y., Wang M., van Wijnen J., Williams R. Global multipollutant modelling of water quality: scientific challenges and future directions. Current Opinion in Environmental Sustainability 2019; 36: 116-125.

Strokal M., Yang H., Zhang Y., Kroeze C., Li L., Luan S., Wang H., Yang S., Zhang Y. Increasing eutrophication in the coastal seas of China from 1970 to 2050. Marine pollution bulletin 2014b; 85: 123-140.

Strokal M.P., Kroeze C., Kopilevych V.A., Voytenko L.V. Reducing future nutrient inputs to the Black Sea. Science of the Total Environment 2014c; 466: 253-264.

Su J., Ji D., Lin M., Chen Y., Sun Y., Huo S., Zhu J., Xi B. Developing surface water quality standards in China. Resources, Conservation and Recycling 2017; 117: 294-303.

Sumei L., ZHANG J., Huiwang G., Zhe L. Historic changes in flux of matter and nutrient budgets in the Bohai Sea. Chinese Society of Oceanography, Acta Oceanologica Sinica 2008; 27.

Sun C., Shen Z., Liu R., Xiong M., Ma F., Zhang O., Li Y., Chen L. Historical trend of nitrogen and phosphorus loads from the upper Yangtze River basin and their responses to the Three Gorges Dam. Environmental Science and Pollution Research 2013; 20: 8871-8880.

Sun H., Shen Y. Agricultural natural resources and regional development in China: Jiangsu science and technology press, 1994.

Sun Q., Miao C., Duan Q. Projected changes in temperature and precipitation in ten river basins over China in 21st century. International Journal of Climatology 2015a; 35: 1125-1141.

Sun X., Xiong S., Zhu X., Zhu X., Li Y., Li B.L. A new indices system for evaluating ecologicaleconomic-social performances of wetland restorations and its application to Taihu Lake Basin, China. Ecological modelling 2015b; 295: 216-226.

Sutton M.A., Bleeker A., Howard C., Erisman J., Abrol Y., Bekunda M., Datta A., Davidson E., de Vries W., Oenema O. Our nutrient world. The challenge to produce more food \& energy with less pollution. Centre for Ecology \& Hydrology, 2013.

Sutton M.A., Oenema O., Erisman J.W., Leip A., van Grinsven H., Winiwarter W. Too much of a good thing. Nature 2011; 472: 159.

Team C.W. Workshop on The Nature and Use of New Socioeconomic Pathways for Climate Change Research. 2011.

Ti C.P., Pan J.J., Xia Y.Q., Yan X.Y. A nitrogen budget of mainland China with spatial and temporal variation. Biogeochemistry 2012; 108: 381-394.

Tian P., Fang X., Wang F., Zhu Y. Use of a minimum environmental Gini Coefficient model on optimizing the allocation plan of total pollutant load in water bodies: a case study at Zhangjiagang river-network plain. China Environmental Science 2014; 34: 801-809.

Tilman D., Balzer C., Hill J., Befort B.L. Global food demand and the sustainable intensification of agriculture. Proceedings of the National Academy of Sciences 2011; 108: 20260-20264.

Tong Y., Bu X., Chen J., Zhou F., Chen L., Liu M., Tan X., Yu T., Zhang W., Mi Z., Ma L., Wang X., Ni J. Estimation of nutrient discharge from the Yangtze River to the East China Sea and the identification of nutrient sources. Journal of Hazardous Materials 2017; 321: 728-736.

Tong Y., Wang X., Zhen G., Li Y., Zhang W., He W. Agricultural water consumption decreasing nutrient burden at Bohai Sea, China. Estuarine, Coastal and Shelf Science 2016; 169: 8594. 
Tong Y., Zhao Y., Zhen G., Chi J., Liu X., Lu Y., Wang X., Yao R., Chen J., Zhang W. Nutrient Loads Flowing into Coastal Waters from the Main Rivers of China (2006-2012). Scientific reports $2015 ; 5$.

UN. Transforming our World: the 2030 Agenda for Sustainable Development Annex A/RES/70/1. United Nations, 2015.

UNEP. Food Systems and Natural Resources. A Report of the Working Group on Food Systems of the International Resource Panel. Westhoek, H, Ingram J., Van Berkum, S., Özay, L., and Hajer M. United Nations Environment Programme, 2016a.

UNEP. A Snapshot of the world's water quality: towards a global assessment. $162 \mathrm{pp}$. United Nations Environment Programme, Nairobi, Kenya, 2016b.

UNFCCC. Adoption of the Paris Agreement. United Nations Framework Convention on Climate Change, 2015.

United Nations. Transforming our world: The 2030 agenda for sustainable development. General Assembley 70 session 2015 .

Valdivia R.O., Antle J.M., Rosenzweig C., Ruane A.C., Vervoort J., Ashfaq M., Hathie I., Tui S.H.-K., Mulwa R., Nhemachena C. Representative agricultural pathways and scenarios for regional integrated assessment of climate change impact, vulnerability and adaptation. Handbook of Climate Change and Agroecosystems 2015: 101-145.

Van Drecht G., Bouwman A., Harrison J., Knoop J. Global nitrogen and phosphate in urban wastewater for the period 1970 to 2050. Global Biogeochemical Cycles 2009; 23.

Van Puijenbroek P., Beusen A., Bouwman A. Global nitrogen and phosphorus in urban waste water based on the shared socio-economic pathways. Journal of environmental management 2019; 231: 446-456.

Van Puijenbroek P., Bouwman A., Beusen A., Lucas P. Global implementation of two shared socioeconomic pathways for future sanitation and wastewater flows. Water Science and Technology 2015; 71: 227-233.

Van Vliet M., van Beek L., Eisner S., Flörke M., Wada Y., Bierkens M. Multi-model assessment of global hydropower and cooling water discharge potential under climate change. Global Environmental Change 2016a; 40: 156-170.

Van Vliet M.T., Wiberg D., Leduc S., Riahi K. Power-generation system vulnerability and adaptation to changes in climate and water resources. Nature Climate Change 2016b; 6: 375.

Van Vuuren D.P., Edmonds J., Kainuma M., Riahi K., Thomson A., Hibbard K., Hurtt G.C., Kram T., Krey V., Lamarque J.-F. The representative concentration pathways: an overview. Climatic change 2011; 109: 5.

Velthof G., Lesschen J., Webb J., Pietrzak S., Miatkowski Z., Pinto M., Kros J., Oenema O. The impact of the Nitrates Directive on nitrogen emissions from agriculture in the EU-27 during 20002008. Science of the Total Environment 2014; 468: 1225-1233.

Velthof G., Oudendag D., Witzke H., Asman W., Klimont Z., Oenema O. Integrated assessment of nitrogen losses from agriculture in EU-27 using MITERRA-EUROPE. Journal of Environmental Quality 2009; 38: 402-417.

Vörösmarty C., Fekete B., Meybeck M., Lammers R. Geomorphometric attributes of the global system of rivers at 30-minute spatial resolution. Journal of Hydrology 2000; 237: 17-39.

Wagner F., Schöpp W., Heyes C. The RAINS optimization module for the clean air for europe (CAFE) programme. 2006. 
Wang C., Liu J.-X., Makkar H.P.S., Wei N.-b., Xu Q.-m. Production level, feed conversion efficiency, and nitrogen use efficiency of dairy production systems in China. Tropical animal health and production 2014a; 46: 669-673.

Wang F., Dou Z., Ma L., Ma W., Sims J., Zhang F. Nitrogen mass flow in China's animal production system and environmental implications. Journal of environmental quality 2010a; 39: 1537-1544.

Wang F., Sims J., Ma L., Ma W., Dou Z., Zhang F. The phosphorus footprint of China's food chain: implications for food security, natural resource management, and environmental quality. Journal of environmental quality 2011a; 40: 1081-1089.

Wang J., Li X., Yan W., Wang F., Ma P. Watershed nitrogen export model related to changing nitrogen balance and hydrology in the Changjiang River basin. Nutrient cycling in agroecosystems 2014b; 98: 87-95.

Wang L. Situation and outlook of feed utilization in dairy industry of China. (In Chinese). Chinese Academy Agri. Sci., Beijing 2005a.

Wang L. Study on production efficiency and milk quality in the main dairy areas. (In Chinese). Chinese Academy Agri. Sci., Beijing 2005b.

Wang L. Modern Chinese pig production. Beijing: Jindun Press, 2007.

Wang L., Chen W. A CMIP5 multimodel projection of future temperature, precipitation, and climatological drought in China. International Journal of Climatology 2014; 34: 2059-2078.

Wang L., Zheng Z., Luo X., Zhang J. The current pollution status and control technology of the Taihu Lake Basin, Jiangsu Province, China. International Journal of Environmental Studies 2010b; 67: 195-205.

Wang M., Kroeze C., Strokal M., Ma L. Reactive nitrogen losses from China's food system for the shared socioeconomic pathways (SSPs). Science of The Total Environment 2017; 605-606: 884-893.

Wang M., Kroeze C., Strokal M., Van Vliet M.T.H., Ma L. Global change can make coastal eutrophication control in China more difficult. Submitted for publication in Earth's Future (Under Review).

Wang M., Ma L., Strokal M., Chu Y., Kroeze C. Exploring nutrient management options to increase nitrogen and phosphorus use efficiencies in food production of China. Agricultural Systems 2018a; 163: 58-72.

Wang M., Ma L., Strokal M., Ma W., Liu X., Kroeze C. Hotspots for Nitrogen and Phosphorus Losses from Food Production in China: A County-Scale Analysis. Environmental Science \& Technology 2018b; 52: 5782-5791.

Wang M., Strokal M., Burek P., Kroeze C., Ma L., Janssen A.B.G. Excess nutrient loads to Lake Taihu: Opportunities for nutrient reduction. Science of The Total Environment 2019a; 664: 865873.

Wang M., Tang T., Burek P., Havlík P., Krisztin T., Kroeze C., Leclère D., Strokal M., Wada Y., Wang Y., Langan S. Increasing nitrogen export to sea: A scenario analysis for the Indus River. Science of The Total Environment 2019b: 133629.

Wang Q.G., Gu G., Higano Y. Toward integrated environmental management for challenges in water environmental protection of Lake Taihu Basin in China. Environmental Management 2006; 37: 579-588.

Wang R.F., An D.G., Hu C.S., Li L.H., Zhang Y.M., Jia Y.G., Tong Y.P. Relationship between nitrogen uptake and use efficiency of winter wheat grown in the North China Plain. Crop and Pasture Science 2011b; 62: 504-514. 
Wang W., Koslowski F., Nayak D.R., Smith P., Saetnan E., Ju X., Guo L., Han G., de Perthuis C., Lin E., Moran D. Greenhouse gas mitigation in Chinese agriculture: Distinguishing technical and economic potentials. Global Environmental Change 2014c; 26: 53-62.

Wiebe K., Lotze-Campen H., Sands R., Tabeau A., van der Mensbrugghe D., Biewald A., Bodirsky B., Islam S., Kavallari A., Mason-D'Croz D. Climate change impacts on agriculture in 2050 under a range of plausible socioeconomic and emissions scenarios. Environmental Research Letters 2015; 10: 085010.

World Bank. Fertilizer consumption, 2016.

Wu H., Kimball J.S., Mantua N., Stanford J. Automated upscaling of river networks for macroscale hydrological modeling. Water Resources Research 2011; 47.

Wu T., Qin B., Brookes J.D., Yan W., Ji X., Feng J. Spatial distribution of sediment nitrogen and phosphorus in Lake Taihu from a hydrodynamics-induced transport perspective. Science of The Total Environment 2019; 650: 1554-1565.

Wu W., Ma B., Uphoff N. A review of the system of rice intensification in China. Plant and Soil 2015.

WU Y.-y., LI Y.-s., LIU W.-j. Study on Gini Coefficient Method of Total Pollutant Load Allocation for Water Bodies [J]. Research of environmental sciences 2006; 2.

Xi J. Environmental Protection Law (In Chinese). In: Congress TSCotNPs, editor, Beijing, China, 2014.

Xi J. Strategy of Rural Vitalization (In Chinese). In: China OotSCotPsRo, editor, Beijing, China, 2018.

Xie J., Zhang X., Xu Z., Yuan G., Tang X., Sun X., Ballantine D. Total phosphorus concentrations in surface water of typical agro-and forest ecosystems in China, 2004-2010. Frontiers of Environmental Science \& Engineering 2014; 8: 561-569.

Xu H., Paerl H.W., Qin B., Zhu G., Hall N.S., Wu Y. Determining critical nutrient thresholds needed to control harmful cyanobacterial blooms in eutrophic Lake Taihu, China. Environmental Science \& Technology 2015a; 49: 1051-1059.

Xu W., Luo X., Pan Y., Zhang L., Tang A., Shen J., Zhang Y., Li K., Wu Q., Yang D. Quantifying atmospheric nitrogen deposition through a nationwide monitoring network across China. Atmospheric Chemistry and Physics 2015b; 15: 12345-12360.

Xu Z., Zhang X., Xie J., Yuan G., Tang X., Sun X., Yu G. Total nitrogen concentrations in surface water of typical agro-and forest ecosystems in China, 2004-2009. PloS one 2014; 9: e92850.

Yan W., Mayorga E., Li X., Seitzinger S.P., Bouwman A. Increasing anthropogenic nitrogen inputs and riverine DIN exports from the Changjiang River basin under changing human pressures. Global Biogeochemical Cycles 2010; 24.

Yang G.Q. Laying manure treatment and resource utilization technology mode (In Chinese). Yunnan Livestock Veterinary 2018; 4: 20-23.

Yang H. Livestock development in China: animal production, consumption and genetic resources. Journal of Animal Breeding and Genetics 2013; 130: 249-251.

Yang J., Strokal M., Kroeze C., Wang M., Wang J., Wu Y., Bai Z., Ma L. Nutrient losses to surface waters in Hai He basin: A case study of Guanting reservoir and Baiyangdian lake. Agricultural Water Management 2019; 213: 62-75.

Yao Q.-Z., Yu Z.-G., Chen H.-T., Liu P.-X., Mi T.-Z. Phosphorus transport and speciation in the Changjiang (Yangtze River) system. Applied Geochemistry 2009; 24: 2186-2194.

Yasin J.A., Kroeze C., Mayorga E. Nutrients export by rivers to the coastal waters of Africa: Past and future trends. Global Biogeochemical Cycles 2010; 24. 
Yilmazel Y., Demirer G. Removal and recovery of nutrients as struvite from anaerobic digestion residues of poultry manure. Environmental technology 2011; 32: 783-794.

Yin K., Harrison P.J. Nitrogen over enrichment in subtropical Pearl River estuarine coastal waters: Possible causes and consequences. Continental Shelf Research 2008; 28: 1435-1442.

Yu C., Huang X., Chen H., Godfray H.C.J., Wright J.S., Hall J., Gong P., Ni S., Qiao S., Huang G. Managing nitrogen to restore water quality in China. Nature 2019: 1.

Yu G., Xue B., Lai G., Gui F., Liu X. A 200-year historical modeling of catchment nutrient changes in Taihu basin, China. Eutrophication of Shallow Lakes with Special Reference to Lake Taihu, China. Springer, 2007, pp. 79-87.

Yuan Q., McIntyre N., Wu Y., Liu Y., Liu Y. Towards greater socio-economic equality in allocation of wastewater discharge permits in China based on the weighted Gini coefficient. Resources, Conservation and Recycling 2017; 127: 196-205.

Zhang F., Chen X., Vitousek P. Chinese agriculture: An experiment for the world. Nature 2013; 497: 33-35.

Zhang F., Cui Z., Chen X., Ju X., Shen J., Chen Q., Liu X., Zhang W., Mi G., Fan M. Integrated nutrient management for food security and environmental quality in China. Advances in agronomy 2012; 116: 1 .

Zhang F., Cui Z., Fan M., Zhang W., Chen X., Jiang R. Integrated soil-crop system management: reducing environmental risk while increasing crop productivity and improving nutrient use efficiency in China. Journal of Environmental Quality 2011; 40: 1051-1057.

Zhang M., Shi X., Yang Z., Yu Y., Shi L., Qin B. Long-term dynamics and drivers of phytoplankton biomass in eutrophic Lake Taihu. Science of The Total Environment 2018; 645: 876-886.

Zhang X., Davidson E.A., Mauzerall D.L., Searchinger T.D., Dumas P., Shen Y. Managing nitrogen for sustainable development. Nature 2015; 528: 51-59.

Zhang X., Wu Y., Liu X., Reis S., Jin J., Dragosits U., Van Damme M., Clarisse L., Whitburn S., Coheur P.-F. Ammonia emissions may be substantially underestimated in China. Environmental Science \& Technology 2017.

Zhao Z., Bai Z., Wei S., Ma W., Wang M., Kroeze C., Ma L. Modeling farm nutrient flows in the North China Plain to reduce nutrient losses. Nutrient Cycling in Agroecosystems 2017: 1-14.

Zheng C. Assessing Environmental Impacts of Chinese Livestock Policies: An Agent-based Approach ((PhD thesis) PhD degree) Wageningen, The Netherlands: Wageningen University, 2013.

Zheng C., Bluemling B., Liu Y., Mol A.P., Chen J. Managing manure from China's pigs and poultry: the influence of ecological rationality. Ambio 2014; 43: 661-672.

Zhou B., Wen Q.H., Xu Y., Song L., Zhang X. Projected changes in temperature and precipitation extremes in China by the CMIP5 multimodel ensembles. Journal of Climate 2014a; 27: 6591-6611.

Zhou F., Chen F., Li Z., Zhu Q., Meng Y., Zhang S. Environmental Characteristics of Nutrients in Pearl River Estuary in Spring 2014 (in Chinese). Journal of Guangdong Ocean University 2018; 38: 42-48.

Zhou F., Shang Z., Ciais P., Tao S., Piao S., Raymond P., He C., Li B., Wang R., Wang X. A new highresolution $\mathrm{N} 20$ emission inventory for China in 2008. Environmental science \& technology 2014b; 48: 8538-8547.

Zhu Z., Jin J. Fertilizer use and food security in China. Plant Nutrition and Fertilizer Science 2013; 19: 259-273. 



\section{Appendices}

The Appendices contain supplementary materials for Chapter 2-7 in this $\mathrm{PhD}$ thesis.

Appendix I: supplementary materials for Chapter 2 "Hotspots for Nitrogen and Phosphorus Losses from Food Production in China: A County-Scale Analysis"

Appendix II: supplementary materials for Chapter 3 "Excess nutrient loads to Lake Taihu: opportunities for nutrient reduction"

Appendix III: supplementary materials for Chapter 4 "Reactive Nitrogen Losses from China's Food System for the Shared Socio-economic Pathways (SSPs)"

Appendix IV: supplementary materials for Chapter 5 "Global change can make coastal eutrophication control in China more difficult"

Appendix V: supplementary materials for Chapter 6 "Exploring Nutrient Management Options to Increase Nitrogen and Phosphorus Use Efficiencies in Food Production of China" Appendix VI: supplementary materials for Chapter 7 "Reducing nutrient pollution in rivers and coastal waters in China: implications for the Sustainable Development Goals"

The supplementary materials from published articles and the article under revision have been adjusted to the $\mathrm{PhD}$ thesis format (e.g., the numbering, formatting). The adjusted versions of the supplementary materials (all above appendices) for this PhD thesis are available on request (mengru.wang@wur.nl). The published versions of the supplementary materials are available online with the published articles. 



\section{Summary (English)}

China has been developing fast over the last decades. Intensive human activities in food production and urbanization have led to increasing nitrogen $(\mathrm{N})$ and phosphorus (P) losses to water systems, causing freshwater and coastal water pollution. Reducing water pollution is important in China, especially given its commitments to the Sustainable Development Goals (SDGs) (e.g., SDGs 6 - "clean water and sanitation" and 14 - "life below water"). Nutrient models have been used to analyze the causes of nutrient pollution in water systems and to explore solutions for China. Existing modeling studies focus on either nutrient flows in human activities (e.g., food production) at administrative (e.g., province, country) scales, or on nutrient fluxes from land to water systems at biogeophysical scales (e.g., river basins). However, a better understanding of how human activities at the administrative scales affect nutrient fluxes at bio-geophysical scales of water systems is needed for China.

Nutrient pollution in water systems may continue to increase in the future because of the expected changes in socio-economic development (e.g., population, economy) in China. Climate change may also influence hydrology (e.g., runoff, river discharges), and thus will influence nutrient transport in water systems (e.g., rivers). However, how socio-economic and climatic changes, together, will affect future nutrient pollution in water systems in China is not well studied. Such analysis can be done by implementing the global Shared Socio-economic Pathways (SSPs) and Representative Concentration Pathways (RCPs) in nutrient models for China. Moreover, many policies in China attempt to reduce future water pollution by nutrients. However, limited insights are available on the effectiveness of these policies for reducing future water pollution by nutrients.

The objective of this $\mathrm{PhD}$ thesis is thus to improve our understanding of nutrient pollution in water systems in China, with a focus on the challenges, trends, and solutions. To this end, I formulated six sub-objectives for my research on nutrient pollution in water systems (sub-objectives 2, 4, and 6) from human activities on land (sub-objectives 1, 3, and 5).

Sub-objectives focusing on the challenges:

1. To identify hotspots for nutrient losses from food production (Chapter 2) 
2. To compare nutrient loads to Lake Taihu with the critical nutrient loads of the lake (Chapter 3, case study)

Sub-objectives focusing on the trends:

3. To analyze how socio-economic development affects future nutrient losses from food production (Chapter 4)

4. To analyze how global change affects future river export of nutrients to seas (Chapter 5)

Sub-objectives focusing on the solutions:

5. To explore how current and improved nutrient management affects future nutrient losses from food production (Chapter 6)

6. To explore future scenarios to reduce nutrient pollution in water systems and to meet the SDGs (Chapter 7)

Chapters 2 and 3 focus on current state and challenges to reducing nutrient pollution in water systems in China. I started this research by identifying hotspots for nutrient losses from food production at the county scale (Chapter 2). To this end, I applied a newly developed NUFER-county (NUtrient flows in Food chains, Environment and Resources use) model. I conclude that in the recent past, nutrient losses from food production increased dramatically in China. Between 1990 and 2012, the area of hotspots increased by a factor of 3 for $\mathrm{N}$, and 24 for P. In 2012, hotspots covered less than $10 \%$ of the Chinese land area, but contributed by more than half to the $\mathrm{N}$ and $\mathrm{P}$ losses to the environment. I found that direct discharge of animal manure was an important source of $\mathrm{N}$ and P losses to water systems in the hotspot areas. Most of the hotspots were distributed in the North China Plain where agricultural production is more intensive than in other regions.

Next, I analyzed nutrient loads to Lake Taihu in relation to critical nutrient loads (Chapter 3). I developed and applied the MARINA-Lake (Model to Assess River Inputs of Nutrients to seAs) model to quantify river export of total dissolved N (TDN) and P (TDP) to Lake Taihu. The results of MARINA-Lake were compared to critical nutrient loads to assess whether the nutrient loads may cause eutrophication in the lake. I concluded that to meet critical nutrient loads, river export of TDN and TDP to Lake Taihu needs to be reduced by 46-92\%, depending on the desired level of chlorophyll-a. Results on source attribution show that opportunities for reducing nutrient pollution in Lake Taihu are reducing synthetic fertilizers and improving wastewater treatment. 
Chapters 4 and 5 focus on future trends in nutrient pollution in water systems in China as affected by global change. In Chapter 4, I analyzed how socio-economic development affects future $\mathrm{N}$ losses from food production. I developed a step-wise approach that interprets the global SSP storylines for China's food system. The interpreted SSPs for China were applied in NUFER to quantify reactive $\mathrm{N}(\mathrm{Nr})$ losses from the food system to the environment. The main finding of this chapter is that $\mathrm{Nr}$ losses from food production to water systems may increase if nutrient management does not improve. For example, in SSP3 with limited improvements in nutrient management, $\mathrm{Nr}$ losses to water systems from crop and animal production may be 65\% higher in 2050 than in 2010. Opportunities exist to reduce water pollution in the future, shown by SSP1 in which 71\% lower Nr losses to water systems were quantified in 2050 than in 2010.

In Chapter 5, I analyzed how global change affects future river export of nutrients to seas. I developed a new MARINA 2.0 model that couples MARINA 1.0 and NUFER-county for 12 large rivers in China. MARINA 2.0 quantifies river export of TDN and TDP to seas at the sub-basins scale, by sources, in 2012 and 2050. For 2050, I developed and implemented six scenarios combining the SSPs and RCPs. Results show that future river export of TDN and TDP may be considerably higher (e.g., in SSP3-RCP8.5) or considerably lower (e.g., in SSP1-RCP2.6) than today. Climate change may further increase river export of nutrients by $24 \%$ for TDN, and $16 \%$ for TDP between 2012 and 2050, given the same trends in socio-economic development (SSP2-RCP8.5 versus SSP2-RCP2.6).

Chapters 6 and 7 focus on potential options to reduce nutrient pollution in water systems in China. In Chapter 6, I explored how current policies and improved nutrient management affect future nutrient losses from food production. Three future scenarios describing Business As Usual (BAU), the current policy on "Zero growth in Fertilizer use after 2020" (ZF), and Improved Nutrient Management (INM) were implemented in NUFER. Nutrient use efficiencies in food production and associated nutrient losses to the environment were quantified for 31 provinces and China. The main finding of this analysis was that current policies aimed at zero growth in fertilizer use are not very effective in reducing nutrient pollution from food production in China. In 2050, nutrient use efficiencies are a few percents higher in ZF than in BAU, vary among provinces. $\mathrm{N}$ and $\mathrm{P}$ losses to waters are $8-16 \%$ lower than in BAU but still higher than today. To further reduce nutrient losses to waters, improved nutrient management is needed for China. 
In Chapter 7, I explored future scenarios to reduce nutrient pollution in water systems and to meet SDGs 6 and 14. TDN and TDP inputs to six Chinese rivers and river export of TDN and TDP to seas by sub-basins in 2050 were quantified for six future scenarios using MARINA 2.0. Two indicators were identified for assessing SDGs 6 and 14. I found that it is difficult to meet SDGs 6 and 14 in the baseline scenario. Future scenarios combing improved nutrient management in agriculture and sewage systems, healthy and efficient food consumption, and climate mitigation may be effective to meet SDGs 6 and 14 . Following these scenarios, more than $30 \%$ of the sub-basins of six large rivers in China may be able to meet SDG 6 at their outlets, as indicated by N and P concentrations. Three large rivers (the Huai, Yangtze, and Pearl rivers) may meet SDG 14 with low potential to cause coastal eutrophication, indicated by negative ICEP (Indicator for Coastal Eutrophication Potential) values. I also found that future scenarios contributing to SDG 6 and 14 may also contribute to other SDGs, focusing on zero hunger (SDG 2), responsible consumption and production (SDG 12), climate action (SDG 13), and others.

Last, I reflected on the research in this thesis, drew five main lessons for future nutrient modeling, and discussed the implications of this thesis on future policies (Chapter 8). The main lessons are as follows: 1) linking NUFER and MARINA models can help to better explore solutions to reduce nutrient pollution in water systems at administrative and biogeophysical scales, 2) preferred spatial scales of modeling depending on the research objectives, 3 ) indicators can help to better understand and communicate the modeling results, 4) combining different types of scenarios is useful in exploring solutions in environmental modeling, and 5) the modeling approach in this thesis can be used to analyze nutrient pollution in water systems in other world regions.

The development of recent environmental policies in China confirms two main findings of this thesis: 1) nutrient pollution in water systems has increased dramatically in China, and 2) direct discharge of animal manure and human waste are the important sources of nutrient pollution in water systems in China. My $\mathrm{PhD}$ thesis supports the formulation of future environmental policies for water pollution control in China. The linked NUFERMARINA approach provides novel insights to nutrient pollution at both administrative (e.g., county, province, and country) and bio-geophysical (e.g., sub-basin) scales. Such insights are useful for formulating policies that are often implemented at administrative scales to reduce water pollution at bio-geophysical scales. The potential options for 
reducing water pollution proposed in this thesis can be considered by policymakers as a starting point. Further improvement of these options using the participatory approach can be done to account for different perspectives and uncertainties for policy implementation. Quantitative indicators for water pollution in this thesis are helpful in understanding and communicating the modeling results with policymakers and stakeholders. All this hopefully will contribute to reducing water pollution by nutrients, and to meet the SDGs 6 and 14 in China. 



\section{Samenvatting (Nederlands)}

China heeft zich de laatste decennia snel ontwikkeld. Intensieve menselijke activiteiten in voedselproductie en verstedelijking hebben geleid tot toenemende verliezen van stikstof (N) en fosfor (P) naar watersystemen, waardoor zoetwater- en kustwatersystemen vervuilen. Het verminderen van watervervuiling is een belangrijke doelstelling voor China, vooral gezien toezeggingen aan de Sustainable Development Goals (SDG's) (bijv. SDG's 6 - "schoon water en sanitaire voorzieningen" en 14 - "leven onder water"). Met behulp van nutriëntenmodellen zijn de oorzaken van nutriëntenvervuiling in watersystemen geanalyseerd en oplossingen voor China zijn verkend. Bestaande modelstudies richten zich op ofwel nutriëntenstromen in menselijke activiteiten (bijv. voedselproductie) op bestuurlijke (bijv. provincie, land) schalen, of op nutriëntenstromen van land naar watersystemen op bio-geofysische schalen (bijv. stroomgebieden). Er is echter een beter begrip nodig van hoe de menselijke activiteiten op de bestuurlijke schaal de nutriëntenstromen op bio-geofysische schaal van watersystemen in China beïnvloeden.

De vervuiling van watersystemen met nutriënten zal in te toekomst mogelijk verder toenemen vanwege de verwachte veranderingen in de sociaaleconomische ontwikkeling (bijv. bevolking, economie) in China. Klimaatverandering kan ook de hydrologie beïnvloeden (bijv. afspoeling, rivierafvoeren) en zal dus het nutriëntentransport in watersystemen (bijv. rivieren) beïnvloeden. Hoe sociaaleconomische en klimatologische veranderingen samen de toekomstige nutriëntenvervuiling van watersystemen in China beïnvloeden, is echter niet goed bestudeerd. Een dergelijke analyse kan worden gedaan door de zogenoemde monidale "Shared Socio-economic Pathways (SSP's)" en de zogenoemde "Representative Concentration Pathways" (RCP's) in nutriëntenmodellen voor China te implementeren. Bovendien proberen veel beleidsmaatregelen in China toekomstige waterverontreiniging door nutriënten te verminderen. Er zijn echter beperkte inzichten beschikbaar over de effectiviteit van dit beleid voor het verminderen van toekomstige waterverontreiniging door nutriënten.

Het doel van dit proefschrift is dan ook om ons begrip van nutriëntenverontreiniging in watersystemen in China te verbeteren, met een focus op de uitdagingen, trends, en oplossingen. Daartoe formuleerde ik zes subdoelstellingen voor mijn onderzoek naar nutriëntenverontreiniging 
in watersystemen (subdoelstellingen 2,4 en 6) door menselijke activiteiten op het land (subdoelstellingen 1, 3 en 5).

Subdoelstellingen gericht op de uitdagingen:

1. Hotspots identificeren voor nutriëntenverliezen door voedselproductie (Hoofdstuk 2)

2. Nutriëntenbelastingen van het meer Taihu vergelijken met de kritische nutriëntenbelastingen van het meer (Hoofdstuk 3 , casestudie)

Subdoelstellingen gericht op de trends:

3. Analyseren hoe sociaaleconomische ontwikkeling de toekomstige nutriëntenverliezen door voedselproductie beïnvloedt (Hoofdstuk 4)

4. Analyseren hoe wereldwijde verandering de toekomstige rivierexport van nutriënten naar zeeën beïnvloedt (Hoofdstuk 5)

Subdoelstellingen gericht op de oplossingen:

5. Onderzoeken hoe huidig en verbeterd nutriëntenbeheer de toekomstige nutriëntenverliezen van voedselproductie beïnvloedt (Hoofdstuk 6)

6. Toekomstscenario's verkennen om de nutriëntenverontreiniging van watersystemen te verminderen en te voldoen aan de SDG's (Hoofdstuk 7)

Hoofdstukken 2 en 3 richten zich op de huidige staat en de uitdagingen voor het reduceren van nutriëntenverontreiniging in watersystemen in China. Ik begon dit onderzoek met het identificeren van hotspots voor nutriëntenverliezen door voedselproductie op provinciale schaal (Hoofdstuk 2). Hiertoe heb ik een nieuw ontwikkeld NUFER-county (Nutrient flows in Food chains, Environment and Resources use) model toegepast. Ik concludeer dat in het recente verleden de nutriëntenverliezen door voedselproductie in China dramatisch zijn toegenomen. Tussen 1990 en 2012 nam het areaal hotspots toe met een factor 3 voor $\mathrm{N}$ en 24 voor P. In 2012 bestreken hotspots minder dan 10\% van het Chinese landoppervlak, maar droegen meer dan de helft bij aan de N- en P-verliezen naar het milieu. Ik ontdekte dat directe lozing van dierlijke mest een belangrijke bron was van $\mathrm{N}$ - en P-verliezen naar watersystemen in de hotspotgebieden. De meeste hotspots liggen verspreid in de vlakte van Noord-China (Chinees Laagland), waar de landbouwproductie intensiever is dan in andere regio's.

Vervolgens analyseerde ik nutriëntenbelastingen naar het meer Taihu in relatie tot kritische nutriëntenbelastingen (Hoofdstuk 3). Ik ontwikkelde en paste het MARINA-Lake (Model to Assess River Inputs of Nutrients to seAs) model toe om de rivierexport van totaal opgelost N (TDN) en P (TDP) naar het meer Taihu te kwantificeren. De resultaten 
van MARINA-Lake werden vergeleken met kritische nutriëntenbelastingen om te beoordelen of de nutriëntenbelastingen eutrofiëring in het meer kunnen veroorzaken. Ik concludeerde dat om aan kritieke nutriëntenbelastingen te voldoen, de rivierexport van TDN en TDP naar het meer Taihu met 46-92\% moet worden verminderd, afhankelijk van het gewenste niveau van chlorofyl-a. Onze resultaten laten zien dat er, om de nutriëntenverontreiniging in het meer Taihu te verminderen, kansen liggen in de vermindering van synthetische meststoffen en het verbeteren van de afvalwaterzuivering.

De Hoofdstukken 4 en 5 richten zich op toekomstige trends in de nutriëntenvervuiling in watersystemen in China die worden beïnvloed door mondiale veranderingen. In Hoofdstuk 4 heb ik geanalyseerd hoe sociaaleconomische ontwikkeling toekomstige Nverliezen door voedselproductie beïnvloedt. Ik ontwikkelde een stapsgewijze aanpak die de wereldwijde SSP-verhaallijnen voor het voedselsysteem van China interpreteert. De geïnterpreteerde SSP's voor China werden in NUFER toegepast om reactieve N (Nr) verliezen van het voedselsysteem naar het milieu te kwantificeren. De belangrijkste bevinding van dit Hoofdstuk is dat Nr-verliezen naar watersystemen door voedselproductie kunnen toenemen als het nutriëntenbeheer niet verbetert. Bijvoorbeeld onder SSP3 met beperkte verbetering van nutriëntenbeheer, zullen Nr-verliezen naar watersystemen uit de gewas- en veeteelt 65\% hoger zijn in 2050 dan in 2010. Er zijn mogelijkheden om in de toekomst waterverontreiniging te verminderen, blijkt uit SSP1 waarbij 71\% lagere $\mathrm{Nr}$-verliezen naar watersystemen werden gekwantificeerd voor 2050 dan in 2010.

In Hoofdstuk 5 heb ik geanalyseerd hoe mondiale veranderingen de toekomstige rivierexport van nutriënten naar zee beïnvloed. Ik ontwikkelde een nieuw MARINA 2.0model dat MARINA 1.0 en NUFER-county koppelt voor 12 grote rivieren in China. MARINA 2.0 kwantificeert rivierexport van TDN en TDP naar zeeën op de schaal van deelstroomgebieden, per bron, in 2012 en 2050. Voor 2050 ontwikkelde en implementeerde ik zes scenario's die de SSP's en RCP's combineren. De resultaten tonen aan dat de toekomstige rivierexport van TDN en TDP aanzienlijk hoger kan zijn (bijv. in SSP3-RCP8.5) of aanzienlijk lager (bijv. in SSP1-RCP2.6) dan vandaag de dag. Klimaatverandering kan de rivierexport van nutriënten verder verhogen met 24\% voor TDN en 16\% voor TDP tussen 2012 en 2050, onder dezelfde trends in sociaaleconomische ontwikkeling (SSP2-RCP8.5 versus SSP2-RCP2.6). 
De Hoofdstukken 6 en 7 richten zich op mogelijke opties om de nutriëntenvervuiling van watersystemen in China te verminderen. In Hoofdstuk 6 heb ik onderzocht hoe het huidige beleid en verbeterd nutriëntenbeheer van invloed zijn op toekomstige nutriëntenverliezen door voedselproductie. Ik heb drie toekomstscenario's geïmplementeerd in NUFER: Business As Usual (BAU), het huidige beleid voor "Nulgroei in gebruik van meststoffen na 2020" (ZF) en verbeterd nutriëntenbeheer (INM). Efficiëntie van het gebruik van nutriënten bij de voedselproductie en de daarmee gepaard gaande verliezen van nutriënten naar het milieu werden gekwantificeerd voor 31 provincies en China. De belangrijkste bevinding van deze analyse was dat het huidige beleid gericht op nulgroei in het gebruik van meststoffen niet voldoende effectief is in het verminderen van nutriëntenvervuiling door voedselproductie in China. Ik laat zien dat de "Nutrient use efficiency" enkele procenten hoger is voor scenario ZF dan in BAU voor 2050, afhankelijk van de provincie. N- en P-verliezen naar wateren zijn 8-16\% lager dan in BAU, maar nog steeds hoger dan vandaag de dag. Om het nutriëntenverlies naar wateren verder te verminderen, is een verbeterd nutriëntenbeheer voor China nodig.

In Hoofdstuk 7 heb ik toekomstige scenario's onderzocht om de nutriëntenvervuiling van watersystemen te verminderen en om te voldoen aan SDG's 6 en 14. TDN- en TDPinvoer naar zes Chinese rivieren en de rivierexport van TDN en TDP naar zeeën door deelstroomgebieden in 2050 werden gekwantificeerd voor zes toekomstscenario's met MARINA 2.0. Twee indicatoren werden geïdentificeerd voor het beoordelen van SDG's 6 en 14. Ik laat zien dat de SDG's 6 en 14 slecht te behalen zijn in het basisscenario. Toekomstige scenario's die uitgaan van een verbeterd nutriëntenbeheer in de landbouw en afvalwatersystemen, gezonde en efficiënte voedselconsumptie en klimaatmitigatie, kunnen effectief zijn om te voldoen aan SDG's 6 en 14 . Volgens deze scenario's kunnen meer dan $30 \%$ van de deelstroomgebieden van zes grote rivieren in China aan SDG 6 voldoen, zoals de N- en P-concentraties bij de uitgangen van de deelstroomgebieden laten zien. Drie grote rivieren (de Huai, Yangtze, en Pearl rivieren) kunnen aan SDG 14 voldoen met geringe kusteutrofiëring, zoals een negatieve ICEP laat zien (Indicator voor kusteutrofiëring Potentiële). Ik heb ook geconstateerd dat toekomstige scenario's die bijdragen aan SDG 6 en 14 ook kunnen bijdragen aan andere SDG's, gericht op zero honger (SDG 2), verantwoorde consumptie en productie (SDG 12), klimaatactie (SDG 13) en andere. 
Tot slot heb ik gereflecteerd op het onderzoek in dit proefschrift en daar vijf hoofdlessen uitgetrokken voor toekomstige modellering van nutriënten en ik heb de implicaties van dit proefschrift voor toekomstig beleid bediscussieerd (Hoofdstuk 8). De belangrijkste lessen zijn als volgt: 1) het koppelen van NUFER en MARINA modellen kan helpen om beter oplossingen te verkennen om nutriëntenverontreiniging in watersystemen te verminderen op bestuurlijke en bio-geofysische schalen, 2) de voorkeur voor de ruimtelijke schaal van modellen hangt samen met de onderzoeksdoelstellingen, 3) indicatoren kunnen helpen om de modelleringsresultaten beter te begrijpen en te communiceren ,4) het combineren van verschillende soorten scenario's is nuttig bij het verkennen van oplossingen door milieumodellering, en 5) de modelleringsbenadering in dit proefschrift kan worden gebruikt om nutriëntenverontreiniging in watersystemen in andere werelddelen te analyseren.

De ontwikkeling van recent milieubeleid in China bevestigt twee belangrijke bevindingen van dit proefschrift: 1) de nutriëntenvervuiling in watersystemen is dramatisch toegenomen in China, en 2) directe lozing van dierlijke mest en afvalwater zijn de belangrijke bronnen van nutriëntenvervuiling in watersystemen in China. Mijn proefschrift ondersteunt de formulering van toekomstig milieubeleid om watervervuiling in China te bestrijden. De gekoppelde NUFER-MARINA-benadering biedt nieuwe inzichten in nutriëntverontreiniging op zowel bestuurlijke (bijv. gemeente, provincie en land) als bio-geofysische (bijv. deelstroomgebieden) schalen. Dergelijke inzichten zijn nuttig voor het formuleren van beleid dat vaak op bestuurlijke schaal wordt geïmplementeerd om waterverontreiniging op bio-geofysische schaal te verminderen. De mogelijke opties voor het verminderen van watervervuiling die in dit proefschrift zijn voorgesteld, kunnen door beleidsmakers als een uitgangspunt worden beschouwd. Verdere verbetering van deze opties kan worden bereikt door middel van een participatieve aanpak die rekening houdt met verschillende perspectieven en onzekerheden voor de uitvoering van het beleid. Kwantitatieve indicatoren voor watervervuiling in dit proefschrift zijn nuttig bij het begrijpen en communiceren van de modelresultaten met beleidsmakers en belanghebbenden. Hopelijk draagt dit alles bij aan het verminderen van watervervuiling door nutriënten en aan het behalen van de SDG's 6 en 14 in China. 


\section{Summary (中文)}

中国经济在过去的几十年中迅速发展, 高度密集的农业活动和快速的城市化进程导致 大量氮、磷等养分向水体流失, 从而引发河流、湖泊和近岸海域等水系统的环境污染 问题。与此同时, 为了在中国实现联合国制定的可持续发展目标的计划（例如, 可持 续发展目标 6-“清洁饮水和卫生设施”和 14-“水下生物”），如何减少氮、磷对水体的污 染已成为中国当前函待解决的重大问题之一。目前, 许多研究应用养分模型分析中国 水系统中氮、磷的来源, 进而探索减少氮、磷对水体污染的解决方案。大部分现有的 养分模型侧重于行政尺度上（例如省, 国家）人类活动（例如粮食生产）中的养分流 动, 或在生物地球物理尺度 (例如流域) 上核算从陆地到水体的养分排放通量。在此 基础上, 中国仍然需要更好地了解行政尺度上的人类活动如何影响生物地球物理尺度 上陆地向水体的养分排放通量。

中国社会经济 (例如人口, 经济) 在未来几十年可能保持增长的趋势, 从而导致水体 中氮、磷污染的持续增加。同时, 气候变化也将影响未来水文 (例如地表径流, 河流 流量) 的演变, 进而影响养分在水体 (例如河流) 中的传输过程。但是, 中国未来的 社会经济发展和气候变化将如何共同影响水体中氮、磷污染尚不明确, 该问题可通过 将全球共享的社会经济情景（SSP）和气候变化的代表性浓度情景（RCP）应用于养分 流动模型来进行分析回答。此外, 中国近年来制定了多项政策以试图减少未来氮、磷 等养分对水体的污染，但是这些政策的有效性尚不明确。

因此, 本博士学位论文的主要目的是提高对中国水系统中氮、磷污染的理解, 侧重于 分析该问题目前所面临的挑战, 未来的变化趋势以及解决方案。为此制定了以下六个 研究目标:

聚焦当前挑战的研究目标:

1. 定位中国粮食生产高养分损失的热点地区（第二章）

2. 比较太湖流域向太湖的养分输入和太湖水华爆发的营养盐负荷阈值 (第三章, 案例 研究)

预测未来趋势的研究目标:

3. 分析未来社会经济发展如何影响中国粮食生产中的养分流失 (第四章)

4. 分析未来全球变化如何影响中国河流向海洋的氮、磷输出 (第五章) 
专注解决方案的研究目标:

5. 探索当前和优化的养分管理措施如何影响未来中国粮食生产中的养分流失 (第六章)

6. 探索减少未来水体中氮、磷污染并达到可持续发展目标的解决方案 (第七章)

第二章和第三章的研究聚焦中国水体养分污染的现状和挑战。首先, 第二章开发

NUFER（食物链, 环境和资源使用中的养分流动）县域模型并应用于中国 2000 多个县, 定位粮食生产中高养分流失的热点地区。研究结果表明, 近年来中国粮食生产中氮和 磷的流失急剧增加。自 1990 年到 2012 年之间, 热点地区的氮损失量增加了 3 倍, 磷 损失量增加了 24 倍; 2012 年, 热点地区面积以不足 10\%的中国国土面积, 贡献了全 国一半以上的氮、磷损失; 动物粪便向水体的直接排放是热点地区水体氮、磷污染的 重要来源; 大多数热点地区分布在农业生产更为密集的华北平原。

接下来, 本论文分析比较了太湖流域向太湖的养分输入量和太湖水华爆发的营养盐负 荷阈值（第三章）。第三章开发并应用 MARINA-Lake 模型量化太湖流域向太湖的总溶 解氮 (TDN) 和磷 (TDP) 的输入, 然后将 MARINA-Lake 结果与太湖水华爆发的营养 盐负荷阈值进行比较, 以评估太湖流域向太湖的氮、磷输入量是否可能导致湖泊富营 养化。研究结果表明, 为了避免超过营养盐负荷阈值引发湖泊富营养化, 太湖流域向 太湖的 TDN 和 TDP 输入量需要减少 46-92\%, 具体的减少量取决于不同水质要求所需 的叶绿素 a 水平。来源归因结果表明, 减少合成肥料的使用和改善废水处理设施是减 少太湖氮、磷污染的主要方向。

第四章和第五章重点讨论了受全球变化影响的情况下未来中国水系统氮、磷污染趋势。 在第四章中, 重点分析了未来社会经济发展如何影响中国粮食生产中的活性氮 $(\mathrm{Nr})$ 损失。基于全球社会经济发展 SSP 情景, 开发设计了针对中国未来食物系统的 SSP 情 景, 并将该情景应用于在 NUFER 模型中, 量化从食物系统到环境的 $\mathrm{Nr}$ 损失。研究发 现, 如果未来食物系统中的养分管理不予改善, 从粮食生产到水系统的 $\mathrm{Nr}$ 损失可能会 增加。例如, 在养分管理措施有限的 SSP3 情景中, 到 2050 年, 作物和动物生产向水 系统的 $\mathrm{Nr}$ 损失可能比 2010 年增加 $65 \%$; 未来存在减少水污染的机会存在于 SSP1 情 景中, 在该情境下, 到 2050 年, 作物和动物生产向水系统的 $\mathrm{Nr}$ 损失可能比 2010 年 低 $71 \%$ 。

第五章主要分析了全球变化如何影响未来河流向海洋的养分输出。通过耦合 MARINA 1.0 和 NUFER-county 模型, 开发 MARINA 2.0 模型, 并将其运用于中国 12 条主要河 
流。MARINA 2.0 模型在子流域尺度上, 估算了 2012 年和 2050 年河流向海洋的 TDN 和 TDP 输出量以及主要的养分来源。本研究结合社会经济发展 SSP 情景和气候变化 RCP 情景开发了六个 SSP-RCP 情景。结果表明, 未来中国河流向海洋的 TDN 和 TDP 输 出可能远高于当前水平 (例如, 在 SSP3-RCP8.5 中) 或低于现有水平 (例如, 在 SSP1RCP2.6 中）。考虑到相同的社会经济发展趋势（SSP2-RCP8.5 与 SSP2-RCP2.6）, 在 2012 年至 2050 年之间, 气候变化可能会导致未来中国河流向海洋的 TDN 进一步增加 $24 \%$, TDP 进一步增加 $16 \%$ 。

第六章和第七章重点讨论了减少中国水系统中氮、磷污染的潜在措施。第六章探讨了 中国当前的政策和进一步改善的养分管理措施将如何影响未来中国作物和动物生产中 的养分流失。通过三个未来情景在 NUFER 模型中的分析, 对 31 个省和全国的粮食生 产中的养分利用效率及相关的氮磷损失进行了量化, 三个情景分别描述了“一切照旧” （BAU），“2020年后化肥使用的零增长”（ZF）和“改良养分管理”（INM）。研究结 果表明, 目前的化肥使用量零增长政策在减少中国粮食生产中的氮磷流失方面不是很 有效。到 2050 年, ZF 情景的养分利用效率比 BAU 高出几个百分点, 各个省份的养分 利用效率有所不同; ZF 情景中粮食生产向水体的氮、磷损失比 BAU 低 8-16\%, 但仍 比目前水平高; 为了进一步减少养分流失, 中国需要进一步改善作物和动物生产中的 养分管理措施。

第七章着重探讨了如何降低中国未来水体的养分污染并达到可持续发展目标 6 和 14 的 方案。运用 MARINA 2.0 模型和情景分析的方法, 量化 2050 年人类活动向中国六大河 流的 TDN 和 TDP 输入量, 以及各子流域向沿海河口的 TDN 和 TDP 输出。同时, 提出 了两个用于评估可持续发展目标 6 和 14 数量指标。研究发现, 在不提高养分管理的情 景中很难在中国实现可持续发展目标 6 和 14; 未来, 结合农业和污水处理系统中的养 分优化管理、健康和有效的食物消费以及减缓气候变化等措施将有可能有效地实现可 持续发展目标 6 和 14; 在采取上述措施的情境中, 在中国六大流域中, $30 \%$ 以上的流 域在其子流域的河口也许可以实现可持续发展目标; 同时三个大型河流（淮河, 长江 和珠江）河口的 ICEP（近岸海域富营养化潜力指标）值为负, 显示其发生近岸海域富 营养化的可能性很低, 因此可能实现可持续发展目标 14; 同时结果还发现, 未来有利 于实现可持续发展目标 6 和 14 的情景也可能有利于实现其他可持续发展目标, 例如减 
少饥饿（可持续发展目标 2），负责任的消费和生产（可持续发展目标 12），气候行 动（可持续发展目标 13）等等。

最后, 通过对本论文研究的反思, 为未来的养分模型发展总结了五点启示, 并讨论本 论文对中国未来水污染防治政策的影响（第 8 章）。主要的启示如下：1）将 NUFER 和 MARINA 模型耦合起来将有利于更好地探索未来的解决方案, 以减少行政尺度和生 物地球物理尺度上水体的养分污染；2）模型的空间尺度应根据研究目标决定；3）评 价指标可以帮助更好地理解和传达模型结果；4）在模型中运用不同类型的情景有助于 探索环境问题的解决方案；5）本论文中的研究方法可用于分析其他国家水体养分污染 问题。

中国近年来环境政策的发展证实了本论文的两个主要发现：1）中国水体的养分污染急 剧增加; 2) 动物粪便和人类粪便的直接排放是水体养分污染的重要来源。本博士论文 有助于未来中国水污染防治环境政策的制定。耦合的 NUFER-MARINA 方法为行政（例 如县, 省和国家) 和生物地球物理（例如子流域）尺度上的水体养分污染提供了新颖 的见解。这些见解对于在行政尺度上制定和实施政策以减少生物地球物理尺度的水体 污染十分有益。决策者可以以本文提出的减少水污染的潜在措施作为出发点, 通过参 考利益相关者的意见进一步优化这些措施, 以考虑政策实施的不同观点和不确定性。 另外, 本博士论文的水污染定量指标有助于理解和交流环境模型的结果。 


\section{Acknowledgments}

On $5^{\text {th }}$ November 2014, I started my PhD in Wageningen. This has been an exciting as well as challenging journey. I became a more knowledgeable, independent and confident researcher, in the meantime also a wife and a mother. I have gone through this journey not by myself alone, but with help from many others. Here I would like to thank all those people for accompanying and supporting me over the past years.

First, my supervisor and promotor Carolien Kroeze. Carolien, you are a wonderful supervisor! You are knowledgeable, critical, strategic and always supportive! You have taught me many things and gave me a lot of opportunities to develop myself to be a good researcher, planner, presenter and supervisor. What I appreciate the most is that you helped me to see the potential of myself and always encouraged me when I was low or unconfident. In Chinese, we have saying: 千里马常有, 而伯乐不常有- pearls are everywhere, however seldom can they be seen. I am so grateful that you found me as a 'pearl'!

Next, I would like to thank my Chinese promotor Lin Ma. Lin, you supported me in many different ways. You were always there whenever I had questions or would like to have discussions. You provided me plenty of opportunities to visit China and discuss with the Chinese experts on my research. Your comments always inspired me and triggered me to think more and to improve my work. And you are such a nice person, who always smiles and supports.

My deepest appreciation goes to Miss Nutrients (or Nutrient Sisters), our China team! I am so grateful and proud to be part of this team. Our China team started with Carolien, Maryna and I. In 2013, Maryna and I had our first trip to China. We visited rural areas to see nutrient management in Chinese agriculture, and urban areas for the wastewater treatment plants. It was such a nice and special trip that I will never forget. The team has been growing fast in the last years. Annette, Ang, Coco, Jing and Xuanjing joined us. Thank you all for the wonderful trips to China and the fruitful discussions in the past years. Thank Annette and Maryna for being my paranymphs. I am so proud and honored to have two my paranymphs both granted with Veni.

Furthermore, I would like to thank the Water Systems and Global Change (WSG) and Environmental Systems Analysis (ESA) groups for supporting my PhD in the past years. 
Thanks to all colleagues for talks, coffee breaks, group outings, Friday-afternoon drinks and lunch meetings: Carolien, Fulco, Erik, Michelle, Nynke, Iwan, Bert, Ronald, Saskia, Wietse, Jantsje, Bart, Wouter, Maria, Joreen, Spyros, Eveline, Annette, Maryna, Long, Fuhong, Maartje, Patty at WSG; and Karen, Arnold, Sophie, Lars, Wim, Dolf, Andre, Bas, Lenny, Rob, Wichertje, Mathilde at ESA. Special thanks to Rik for the useful suggestions during the SENSE Honors Program through which I got funding for my PhD. Thank all the fellow PhDs for the nice accompany during my PhD: Bram, Richard, Dianneke, Eva Kleingeld, Eva Boon, Wouter, Talardia, Geoffrey, Justine, Jikke, Coco, Ang, Jing, Xuanjing, Jolijn, Emmanuel, Uthpal, Nancy at WSG; Lucie, Lena, Sarahi, Shahid, Confidence, Cheng, Eka, Leonardo, Halima, Alexey, Momo, Jillian, Arritta, Kanokwan at ESA. Special thanks to Dianneke and Annette for translating my thesis summary into Dutch, and to Jing for suggestions to my summary in Chinese. Thank Momo very much for involving me in the trip of the Environmental Technology group to China. During the trip, I gained more knowledge of wastewater treatment technologies, which is useful in my modeling work.

Moreover, I participated in the Young Scientists Summer Program (YSSP) at the International Institute for Applied Systems Analysis in 2018. Thanks to my supervisors at IIASA: Ting, Peter and David, for their guidance, support and time during my YSSP. My gratitude to Simon, Yoshihide, Günther, Sylvia, Mikhail, Barbara, Taher, Yusuke, Petr, Wilfried for the discussions and suggestions to my work. Thanks to Yaoping for sharing her calibrated hydrological model for the Indus River. Many thanks to the YSSP coordinators, Brian, Guiying, Joanne, Tanja and Aleksandra for the coordination of the program. Last, I would like to express thanks to all the fellow YSSPers for their support and great accompany in the unbelievable summer of 2018.

I would like to thank all my Chinese friends in China and in the Nederlands for their support. Thanks to He Liu, Lin Zhao, Ruiyao Li, Ping Fang, Xudong Hu and Hui Ding for always being there in China to support and encourage me, and treat me with all the wonderful Chinese food whenever I was back to China. Thanks to those in Wageningen: Fan Zhang, Hui Zhang, Shiyang Wang, Jie Zhao, Huchen Li, Wenjuan Mu, Xuezhen Guo, Jia Wei, Rong Qian, Hao Feng, Aojia Wang, Guanlin Wang, Fan Li, Meixiu Tan, Minjie Chen, Alita, Wenwen Zhang and Jing Li. Your lovely accompanies are very much appreciated especially in those moments when I missed home in China. 
I would not be who I am today without my family. I cannot thank my parents enough for all the support and love they have given me. Dear Mom and Dad, thank you for supporting me to study in the Netherlands. Because of my choice to study abroad, you have to accept that we only meet each other for a few weeks or days a year. I know this was not easy, especially after being a mother myself. Thank you for your understanding and sacrifice in the past years. I would like to dedicate this thesis to you because it is an achievement of our family. I love you!

亲爱的爸爸妈妈, 如果没有你们的支持和爱, 我不会有今天的成绩。感谢你们支持我 来到荷兰学习。因为我的选择, 你们每年只有很少的机会能见到我。我知道这非常的 不容易, 尤其在我成为母亲之后更能体会你们的心情。感谢你们这些年来的牺牲和对 我的理解。这本书, 我想赠予你们, 因为这是我们共同的成就。我爱你们!

Last but not least, my husband Xing. Thank you for your continuous love, support, patience, caring and the wonderful cooking in the past years. You supported me so much by taking care of our son Siheng when I went abroad for conferences and summer programs in China and Austria. I would never finish my PhD without your support. I am so happy that we've got Siheng, who taught me to be more patient, efficient, to appreciate life and be happy. Thank you so much Siheng for being my kid!

Looking back at the journey of my PhD, I really enjoyed it. Thanks to all the great experience in the past years. I will bring them with me on the next journey that just starts! 


\section{About the author}

Mengru Wang was born on 9 February 1990 in Xuzhou, China. In 2008, after high school Mengru started her bachelor in Mechatronics Engineering at Nanjing Forestry University. Soon in 2009, with a strong interest in environmental sciences, she changed her bachelor program to Forestry Sciences. She received a BSc degree with distinction (Cum Laude) in Forestry Sciences in 2012. After that, Mengru went abroad to Wageningen University \& Research (WUR) to

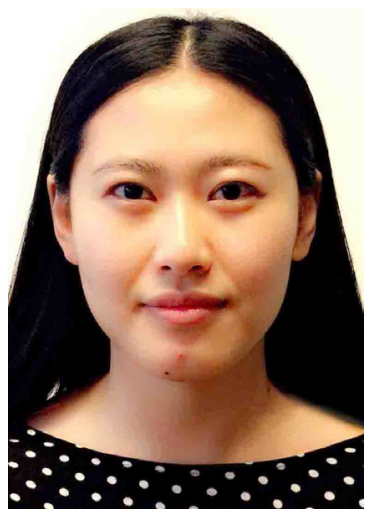
continue her master's study in environmental sciences. In 2014, she received an MSc degree with distinction (Cum Laude) in Environmental Systems Analysis. During her MSc program, she was selected to participate in the honors program for master students in Research School for Socio-Economic and Natural Sciences of the Environment (SENSE). In this program, she wrote a PhD proposal that was granted as the first prize in 2014. With the grant, Mengru started her PhD project at the Water Systems and Global Change, and Environmental Systems Analysis groups of WUR. Her PhD thesis is about reducing nutrient pollution in water systems in China, with a focus on the challenges, trends, and solutions. Mengru developed and applied two new models to quantify nutrient flows from land to water systems in China. During her PhD, Mengru was a member of the PhD council of Wageningen Institute for Environment and Climate Research (WIMEK). She was also active in education and supervision activities. She contributed largely to strengthening and extending the collaboration with China on nutrient management. Mengru attended and presented her research in more than 10 international conferences and symposiums. She participated in the Young Scientists Summer Program (YSSP) at International Institute for Applied Systems Analysis (IIASA) in 2018 (only 53 out of 300 applications were selected). 


\section{List of publications}

Published peer-reviewed articles

2016

Strokal M., Kroeze C., Wang M., Bai Z., Ma L. The MARINA model (Model to Assess River Inputs of Nutrients to seAs): Model description and results for China. Science of The Total Environment 2016; 562: 869-888.

Kroeze C., Gabbert S., Hofstra N., Koelmans A.A., Li A., Löhr A., Ludwig F., Strokal M., Verburg C., Vermeulen L., Van Vliet M.T.H., de Vries W., Wang M., van Wijnen J. Global modelling of surface water quality: a multi-pollutant approach. Current Opinion in Environmental Sustainability 2016; 23: 35-45.

2017

Wang M., Kroeze C., Strokal M., Ma L. Reactive nitrogen losses from China's food system for the shared socioeconomic pathways (SSPs). Science of The Total Environment 2017; 605-606: 884-893.

Strokal M., Kroeze C., Wang M., Ma L. Reducing future river export of nutrients to coastal waters of China in optimistic scenarios. Science of The Total Environment 2017; 579: 517-528.

Zhao Z., Bai Z., Wei S., Ma W., Wang M., Kroeze C., Ma L. Modeling farm nutrient flows in the North China Plain to reduce nutrient losses. Nutrient Cycling in Agroecosystems 2017: 1-14.

2018

Wang M., Ma L., Strokal M., Ma W., Liu X., Kroeze C. Hotspots for Nitrogen and Phosphorus Losses from Food Production in China: A County-Scale Analysis. Environmental Science \& Technology 2018b; 52: 5782-5791.

Wang M., Ma L., Strokal M., Chu Y., Kroeze C. Exploring nutrient management options to increase nitrogen and phosphorus use efficiencies in food production of China. Agricultural Systems 2018a; 163: 58-72.

Bai Z., Lu J., Zhao H., Velthof G.L., Oenema O., Chadwick D., Williams J.R., Jin S., Liu H., Wang M., Strokal M., Kroeze C., Hu C., Ma L. Designing Vulnerable Zones of Nitrogen and Phosphorus Transfers To Control Water Pollution in China. ACS Publications, 2018.

2019

Wang M., Strokal M., Burek P., Kroeze C., Ma L., Janssen A.B.G. Excess nutrient loads to Lake Taihu: Opportunities for nutrient reduction. Science of The Total Environment 2019a; 664: 865-873.

Wang M., Tang T., Burek P., Havlík P., Krisztin T., Kroeze C., Leclère D., Strokal M., Wada Y., Wang Y., Langan S. Increasing nitrogen export to sea: A scenario analysis for the Indus River. Science of The Total Environment 2019b: 133629. 
Chen X., Strokal M., Van Vliet M.T., Stuiver J., Wang M., Bai Z., Ma L., Kroeze C. Multi-scale modeling of nutrient pollution in the rivers of China. Environmental science \& technology 2019.

Yang J., Strokal M., Kroeze C., Wang M., Wang J., Wu Y., Bai Z., Ma L. Nutrient losses to surface waters in Hai He basin: A case study of Guanting reservoir and Baiyangdian lake. Agricultural Water Management 2019; 213: 62-75.

Strokal M., Spanier J.E., Kroeze C., Koelmans A.A., Flörke M., Franssen W., Hofstra N., Langan S., Tang T., Van Vliet M.T.H., Wada Y., Wang M., van Wijnen J., Williams R. Global multi-pollutant modelling of water quality: scientific challenges and future directions. Current Opinion in Environmental Sustainability 2019; 36: 116-125.

Zhuo L., Liu Y., Yang H., Hoekstra A.Y., Liu W., Cao X., Wang M., Wu P. Water for maize for pigs for pork: An analysis of inter-provincial trade in China. Water Research 2019: 115074.

\section{Conference proceedings}

Wang M., Ma L., Strokal M., Chu Y., Kroeze C. Improving nitrogen use efficiency in the Chinese food chain to reduce air and water pollution. Proceedings of the 7 th International Nitrogen Initiative Conference, "Solutions to improve nitrogen use efficiency for the world", Melbourne, Australia, 2016.

Strokal M., Kroeze C., Wang M., Ma L. Increasing nitrogen use efficiency in agriculture reduces future coastal water pollution in China. Proceedings of the 7 th International Nitrogen Initiative Conference, "Solutions to improve nitrogen use efficiency for the world", Melbourne, Australia, 2016b.

\section{In preparation or submitted articles}

Wang M., Kroeze C., Strokal M., Van Vliet M.T.H., Ma L. Global change can make coastal eutrophication control in China more difficult. Submitted for publication in Earth's Future (the revised manuscript has been resubmitted after positve review).

Wang M., Kroeze C., Ma L. Reducing nutrient pollution in rivers and coastal waters in China: implications for the Sustainable Development Goals. To be submitted.

Ma C., Strokal M., Kroeze C., Wang M., Li X., Hofstra N., Ma L. Reducing river export of nutrients and eutrophication in Lake Dianchi in the future. Blue-Green Systems; Accepted.

Tang T., Wang M., Strokal M., Burek P., Leclère D., Krisztin T., Kroeze C., Langan S., Wada Y. Impacts of Climate Change and Socio-economic Development on Future Nitrogen Export: a Comparative Study of Three Large River Basins. In preperation.

Chen X., Strokal M., Kroeze C., Supit I., Wang M., Ma L., Chen X., Shi X. Modelling the contribution of crops to nitrogen pollution in the Yangtze River. In preperation. 


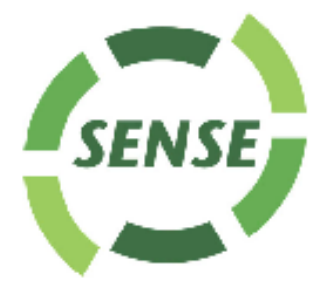

Netherlands Research School for the

Socio-Economic and Natural Sciences of the Environment

\section{I P L O M A for specialised PhD training}

The Netherlands research school for the Socio-Economic and Natural Sciences of the Environment (SENSE) declares that

\section{Mengru Wang}

born on 9 February 1990 in Xuzhou, China

has successfully fulfilled all requirements of the educational PhD programme of SENSE.

Wageningen, 12 February 2020

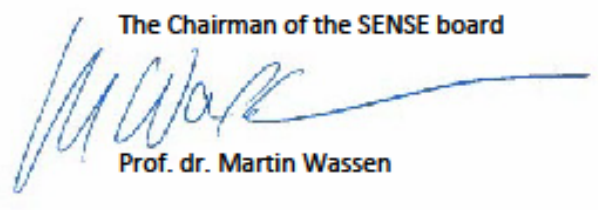

the SENSE Director of Education

The SENSE Research School has been accredited by the Royal Netherlands Academy of Arts and Sciences (KNAW)

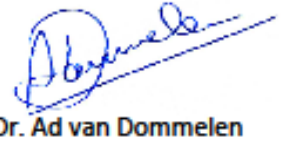

Dr. Ad van Dommelen

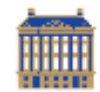

$\begin{array}{lllllllllllllllllllll}K & O & N & \perp & N & K & L & \mathrm{~J} & \mathrm{E} & \mathrm{N} & \mathrm{E} & \mathrm{D} & \mathrm{E} & \mathrm{R} & \mathrm{L} & \mathrm{A} & \mathrm{N} & \mathrm{D} & \mathrm{S} & \mathrm{E}\end{array}$

A K A D E M I E V A N W E T E N S C H A P P E N 


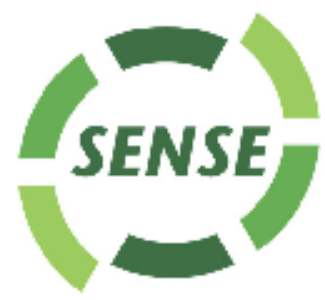

The SENSE Research School declares that Mengru Wang has successfully fulfilled all requirements of the educational PhD programme of SENSE with a work load of $49.2 \mathrm{EC}$, including the following activities:

\section{SENSE PhD Courses}

- Environmental research in context (2015)

- Research in context activity: ‘Organizing a field a trip to China and a symposium on Chinese agriculture development and its impacts on coastal eutrophication' (2015)

\section{Other. PhD and Advanced MSc Courses}

- Information Literacy with EndNote Introduction, Wageningen University (2015)

- Basic statistics, Wageningen University (2016)

- Techniques for writing and presenting a scientific paper, Wageningen University (2016)

- GIS in practise, Wageningen University (2016)

- Reviewing a Scientific Paper, Wageningen University (2016)

- Young Scientists Summer Program, International Institute for Applied Systems Analysis (2018)

\section{Management and Didactic Skills Training}

- Supervising three MSc students with thesis (2014-2019)

- Assisting in the MSc courses 'Seminar interdisciplinarity in scientific research and education' and 'Introduction to environmental systems analysis' and the BSc/MSc course 'Introduction to Global Change' (2015-2018)

- Education Innovation project: developing new course material for computer based teaching (2018)

- Member of WIMEK PhD council (2014-2016)

- Project and time management, Wageningen Graduate Schools (2015)

- Data management planning, Wageningen Graduate Schools (2015)

\section{Selection of Oral Presentations}

- Nitrogen and phosphorus use efficiencies in agricultural production and their effects on water pollution in China. International Interdisciplinary Conference on Land Use and Water Quality, 21-24 September 2015, Vienna, Austria

- Exploring nutrient management options to increase nutrient use efficiencies in agriculture of China. 2nd Global Food Security Conference, 11-14 October 2015, Ithaca, United States

- Future coastal water pollution in China under the Shared Socio-economic Pathways (SSPs) and Representative Concentration Pathways (RCPs). Water Science for Impact Conference, 16-18 October 2018, Wageningen, The Netherlands

\section{SENSE coordinator $\mathrm{PhD}$ education}

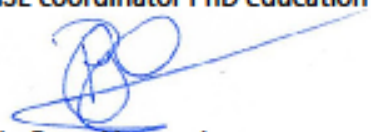

Dr. ir. Peter Vermeulen 
Financial support from Wageningen University for printing this $\mathrm{PhD}$ thesis is highly appreciated.

Thesis cover design: Xing Fu and Mengru Wang

Photograph on the cover page: Ang Li

Printed by GVO drukkers \& vormgevers B.V. (www.gvo.nl) 



\section{Propositions}

1. Changes in climate and society make water pollution control in China more difficult.

(this thesis)

2. Environmental policies should first target one-tenth of the Chinese land that causes over half of the nutrient pollution. (this thesis)

3. Modeling the details does not necessarily contribute to environmental management.

4. To explore future solutions, considering options that are not feasible yet, is indispensable.

5. The impact of research on society is more important than scientific novelty.

6. Having children stimulates efficiency in PhD projects.

Propositions belong to the thesis, entitled

"Reducing nutrient pollution in water systems in China: challenges, trends and solutions"

Mengru Wang

Wageningen, 12 February 2020 
